Pontifícia Universidade $C_{\text {atólica }}$

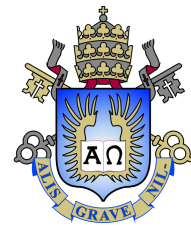

Johnny Felipe Alves de Lima

\title{
O Problema da Quadratura do Círculo no
}

Plano Hiperbólico

Dissertação apresentada como requisito parcial para obtenção do grau de Mestre pelo Programa de Pós-graduação em Matemática (Modalidade Profissional) do Departamento de Matemática do Centro Técnico Científico da PUC-Rio.

Orientador: Prof. José Victor Goulart Nascimento 


\section{O Problema da Quadratura do Círculo no Plano Hiperbólico}

Dissertação apresentada como requisito parcial para obtenção do grau de Mestre pelo Programa de Pós-graduação em Matemática (Modalidade Profissional) do Departamento de Matemática do Centro Técnico Científico da PUC-Rio. Aprovada pela Comissão Examinadora abaixo assinada.

Prof. José Victor Goulart Nascimento

Orientador

Departamento de Matemática - PUC-Rio

Prof. Nicolau Corção Saldanha Departamento de Matemática - PUC-Rio

Prof. Alessandro Gaio Chimenton

Departamento de Matemática - PUC-Rio

Prof. Rodrigo Pereira Pacheco Instituto de Matemática e Estatística - UERJ

Prof. Márcio da Silveira Carvalho

Coordenador Setorial do Centro Técnico Científico - PUC-Rio 
Todos os direitos reservados. É proibida a reprodução total ou parcial do trabalho sem autorização da universidade, do autor e do orientador.

Johnny Felipe Alves de Lima

Licenciado em Matemática pela UERJ-Universidade do Estado do Rio de Janeiro em 2013.

Ficha Catalográfica

Alves de Lima, Johnny Felipe

O Problema da Quadratura do Círculo no Plano Hiperbólico / Johnny Felipe Alves de Lima; orientador: José Victor Goulart Nascimento. - Rio de janeiro: PUC-Rio, Departamento de Matemática , 2017.

v., 147 f: il. color. ; $30 \mathrm{~cm}$

Dissertação (mestrado) - Pontifícia Universidade Católica do Rio de Janeiro, Departamento de Matemática .

Inclui bibliografia

1. Matemática - Teses. 2. Geometria Hiperbólica - Teses. 3. Geometria Hiperbólica; 4. Construções com régua e compasso.. I. Goulart, Victor. II. Pontifícia Universidade Católica do Rio de Janeiro . Departamento de Matemática . III. Título. 


\section{Agradecimentos}

Primeiramente, agradeço a Deus por me ter concedido a oportunidade de existir e de evoluir, mediante diversos desafios, vencidos pouco a pouco nas lides da vida.

Agradeço à minha mãe, Zilda Alves de Lima (in memoriam), inesquecível professora de Matemática, que muito batalhou na dura rotina das escolas públicas para prover, sozinha, o sustento do seus dois filhos.

Agradeço à minha esposa Aline por ter-me incentivado a dar continuidade aos estudos, independentemente das circunstâncias adversas, e por ter suportado as minhas ausências enquanto eu cursava o Mestrado Profissional. Agradeço à minha filha Clarisse pela sua alegria contagiante e por me ensinar a enxergar o mundo pela simplicidade do ponto de vista de uma criança.

Agradeço, também, ao meu orientador, Prof. Victor Goulart, a quem coube a ideia do tema para este trabalho e com quem tive diversas reuniões em que fui introduzido ao universo do pensamento e do ofício matemático.

Agradeço à PUC-Rio pela oportunidade de cursar o Mestrado Profissional em instituição com tão elevado padrão de qualidade. Agradeço a todos os professores pelas valorosas lições ministradas ao longo do curso. 


\section{Resumo}

Alves de Lima, Johnny Felipe; Goulart, Victor. O Problema da Quadratura do Círculo no Plano Hiperbólico. Rio de Janeiro, 2017. 147p. Dissertação de Mestrado - Departamento de Matemática, Pontifícia Universidade Católica do Rio de Janeiro .

A quadratura do círculo é um dos problemas de construtibilidade com régua e compasso legados pela antiguidade clássica e entreteve alguns matemáticos por séculos a fio até que os avanços da Álgebra Moderna demostraram a impossibilidade de tal construção no plano euclideano. Entrementes, desenvolviam-se as chamadas Geometrias Não-Euclideanas, baseadas na substituição do Postulado V de Euclides (axioma das paralelas). O intuito deste trabalho é mostrar como é possível, sob certas condições, produzir um quadrilátero regular e um círculo de mesma área no plano hiperbólico usando apenas régua e compasso (hiperbólicos). Um exemplo é apresentado em detalhe, e as condições necessárias e suficientes para o êxito da construção são apresentadas e discutidas brevemente.

\section{Palavras-chave}

Geometria Hiperbólica; Construções com régua e compasso. 


\section{Abstract}

Alves de Lima, Johnny Felipe; Goulart, Victor (Advisor). The Problem of Squaring the Circle in the Hyperbolic Plane. Rio de Janeiro, 2017. 147p. Dissertação de mestrado - Departamento de Matemática, Pontifícia Universidade Católica do Rio de Janeiro .

Squaring the circle is one of the straightedge and compass constructibility problems whose inception goes back to classical antiquity and that have entertained some mathematicians in the centuries that followed. The development of Modern Algebra has shown beyond doubt that such a construction is impossible in the Euclidean plane. Meanwhile, the so called non-Euclidean Geometries appeared that were based on the replacement of Euclid's fifth postulate (the parallel axiom). The goal of this work is to show how it is possible - under certain constraints - to produce a regular quadrilateral and a circle of same area in the hyperbolic plane by means of (hyperbolic) straightedge and compass alone. An example is presented in full detail, and the necessary and sufficient conditions under which such construction is possible are briefly discussed.

\section{Keywords}

Hyperbolic Geometry; Straightedge and Compass Constructions. 


\section{Sumário}

1 Introdução $\quad 11$

2 Geometria Euclideana $\quad 18$

2.1 Construtibilidade com Régua e Compasso 19

2.1.1 Noções Gerais 19

2.1.2 Três Problemas Clássicos 25

$\begin{array}{lll}2.2 & \text { Geometria Analítica } & 26\end{array}$

2.3 Resposta Moderna aos Problemas Clássicos de Construtibilidade 35

2.4 Inversão Circular 49

3 Geometria Hiperbólica $\quad 63$

3.1 A Geometria Hiperbólica. O Modelo do Disco de Poincaré 63

3.1.1 A Régua e o Compasso Hiperbólicos 65

$\begin{array}{lll}3.1 .2 & \text { Métrica Hiperbólica } & 76\end{array}$

3.1.3 Trigonometria Hiperbólica 84

$\begin{array}{ll}\text { 3.1.4 Construtibilidade no Plano Hiperbólico } & 102\end{array}$

4 Quadratura do Círculo no Plano Hiperbólico 106

4.1 Construção de um Quadrado e de um Círculo de mesma Área no Modelo do Disco de Poincaré com Régua e Compasso Euclideanos 106

4.2 Condições Necessárias e Suficientes para Construir um Quadrado e um Círculo de Mesma Área no Plano Hiperbólico 126

$\begin{array}{lc}\text { Referências bibliográficas } & 146\end{array}$ 


\section{Lista de figuras}

Figura 2.1 Construção - Transferência de Medida de Segmento 20

Figura 2.2 Perpendicular por ponto exterior à reta dada 21

Figura 2.3 Perpendicular por um ponto incidente 22

$\begin{array}{lll}\text { Figura 2.4 Retas paralelas } & 23\end{array}$

$\begin{array}{lll}\text { Figura 2.5 Construção da bissetriz } & 23\end{array}$

Figura 2.6 Retas tangentes por um ponto exterior ao círculo 24

$\begin{array}{lll}\text { Figura 2.7 } & \text { Justificativa para a construção das retas tangentes } & 25\end{array}$

$\begin{array}{lll}\text { Figura 2.8 } & \text { Eixos coordenados } & 27\end{array}$

Figura 2.9 Ilustração - reta prolongada indefinidamente 29

Figura 2.10 Adição e Diferença de Segmentos 33

Figura 2.11 Obtenção do segmento de medida $a b \quad 33$

Figura 2.12 Obtenção do segmento de medida $\frac{b}{a} \quad 33$

Figura 2.13 Obtenção do segmento de medida $\sqrt{a} \quad 34$

Figura 2.14 Ponto Inverso $\quad 50$

Figura 2.15 Inverso de um ponto exterior ao círculo 51

Figura 2.16 Inversão de uma reta $\quad 51$

Figura 2.17 Ângulo entre círculos $\quad 52$

Figura 2.18 Círculos Perpendiculares $\quad 53$

Figura 2.19 Ângulo de Segmento $\quad 54$

Figura 2.20 Teorema-Círculos Perpendiculares e Pontos Inversos $\quad 55$

Figura 2.21 Inversão do círculo $\quad 56$

Figura 2.22 Ilustração - inversão conforme $\quad 58$

Figura 2.23 $O, A$ e $P$ não colineares. $\quad 60$

Figura 2.24 Pontos $O, A$ e $P$ sobre uma mesma reta. 61

Figura 3.1 Retas paralelas e não-secantes no modelo de Poincaré 65

Figura 3.2 Disco de Poincaré — reta hiperbólica por dois pontos 66

Figura 3.3 Disco de Poincaré - paralelas por ponto dado 67

Figura 3.4 Distância no disco de Poincaré 68

Figura 3.5 Inversão por um círculo perpendicular ao bordo do disco de Poincaré $\quad 70$

$\begin{array}{lll}\text { Figura 3.6 Raio do Círculo Hiperbólico } & 71\end{array}$

$\begin{array}{lll}\text { Figura 3.7 } & O, E \text { e } H \text { sobre a mesma reta } & 72\end{array}$

Figura 3.8 Construção do Círculo no Modelo de Poincaré 74

Figura 3.9 Ilustração para a prova de $\mathbf{P} 4$ no modelo do disco de $\begin{array}{ll}\text { Poincaré } & 75\end{array}$

Figura 3.10 Exemplo — distância no modelo do disco de Poincaré $\quad 76$

Figura 3.11 Ãngulo Hiperbólico $\quad 77$

$\begin{array}{lll}\text { Figura 3.12 Angulo de paralelismo } & 78\end{array}$

Figura 3.13 Triângulo Hiperbólico $\quad 79$

Figura 3.14 Ilustração - Defeito do Triângulo Hiperbólico 80

Figura 3.15 Ilustração para prova da proposição da proposição 17 (situação impossível) $\quad 81$

Figura 3.16 Ilustração para a prova do teorema 14 
Figura 3.17 Triângulo Hiperbólico

Figura 3.18 Ilustração para prova da fórmula de Lobatchevski-Bolyai 93

Figura 3.19 Constante de Schweikart

Figura 3.20 Ilustração para a prova do comprimento e da área do círculo

Figura 3.21 Ilustração - Ponto E-construtível e ponto $H$ construtível 103

Figura 3.22 Ilustração — Reta Hiperbólica 103

Figura 4.1 Quadrado e Círculo de Mesma Área - Parte 1 108

Figura 4.2 Quadrado e Círculo de Mesma Área - Parte 2 108

Figura 4.3 Quadrado e Círculo de Mesma Área - Parte 3 109

Figura 4.4 Quadrado e Círculo de Mesma Área - Construção Final 110

Figura 4.5 Justificativa - Construção do Quadrado e do Círculo de Mesma Área - Parte 1

Figura 4.6 Justificativa - Quadrado e Círculo de Mesma Área - Parte 2112

Figura 4.7 Justificativa - Construção do Quadrado e do Círculo de Mesma Área - Parte 3

Figura 4.8 Justificativa - Construção do Quadrado e do Círculo de Mesma Área - Parte 4

Figura 4.9 Justificativa - Construção do Quadrado e do Círculo de Mesma Área - Parte 5

Figura 4.10 Ilustração para a prova da validade das proposições 22, 23 e 24 para ângulos em geral

Figura 4.11 Ilustração para a prova da validade da proposição 25 para um ângulo qualquer

Figura 4.12 Ilustração para a prova da proposição 26 para ângulos quaisquer.

Figura 4.13 Primeira ilustração para a prova da proposição 29 para um ângulo qualquer

Figura 4.14 Primeira ilustração para a prova da proposição 29 para um ângulo qualquer

Figura 4.15 Retas paralelas do ponto de vista axiomático 126

Figura 4.16 Construção de reta paralela do ponto de vista sintético 127

Figura 4.17 Ilustração para justificativa da construção $10 \quad 128$

Figura 4.18 Construção do segmento de paralelismo, dado o ângulo de paralelismo $\rho$

Figura 4.19 Construção dos triângulo retângulo hiperbólico, dados ângulos $\alpha$ e $\beta$

Figura 4.20 Biângulo fechado

Figura 4.21 Construção do biângulo isósceles $A B C_{2} D$, dado o biângulo qualquer $A B C D$

Figura 4.22 Ilustração para justificativa da construção $13 \quad 133$

Figura 4.23 Construção do segmento $r$, dado o ângulo $\theta \quad 134$

Figura 4.24 Construção do ângulo $\theta$, dado o segmento $r$

135

Figura 4.25 Quadrado hiperbólico construído a partir de oito triângulos retângulos congruentes 
"A mente que se abre a uma nova ideia jamais voltará ao seu tamanho original."

EINSTEIN, Albert. 


\section{Introdução}

Como é sabido, o problema da quadratura do círculo - que diz respeito à possibilidade de se construir, com régua e compasso no plano euclideano, um quadrado que tenha mesma área que um círculo dado - foi cogitado pelos gregos ainda na antiguidade clássica e não tem solução na Geometria Euclideana. A resposta negativa a tal problema de construtibilidade é consequência do fato de que $\pi$ é um número transcendente.

No entanto, ainda que possa parecer surpreendente, no contexto da Geometria Hiperbólica - em que P5 é substituído por um postulado alternativo, o qual estabelece que, por um ponto à exterior uma reta, é possível traçar infinitas retas paralelas à reta dada - , pode-se construir, sob algumas condições e com as versões corretas da régua e do compasso, um quadrado e um círculo de mesma área.

O próprio János Bolyai, um dos fundadores da Geometria Hiperbólica, anteviu o fato de que o problema da quadratura da círculo encontraria alguma solução nesta nova Geometria. De fato, seu famoso Apêndice (citado em [1]) tinha por título (em tradução livre): "a absolutamente verdadeira ciência do Espaço exposta independentemente da verdade ou falsidade (a priori indecidível para sempre) do postulado $V$ de Euclides: para o caso de falsidade, com uma quadratura geométrica do círculo."

Mais notável ainda é o fato de que o referido matemático, sem ter um modelo, conseguiu lançar os fundamentos geométricos da prova relativa às condições necessárias e suficientes para que se possa construir um quadrado e um círculo de mesma área no plano hiperbólico, tendo introduzido um ângulo auxiliar $\theta$, que, como será visto no Capítulo 4, terá grande relevância na análise deste problema.

O principal escopo desta dissertação é mostrar que existem quadriláteros regulares e círculos de mesma área no plano hiperbólico, ambos construtíveis com a régua e o compasso intrínsecos a este espaço. Para tanto, será exibida uma construção euclideana do quadrado e do círculo de mesma área no disco de Poincaré, baseada em [2]. Será apresentado, ainda, o teorema de Nestorovich-Jagy, que estabelece as condições necessárias e suficientes para que um quadrilátero regular e um círculo de mesma 
área possam ser construídos no plano hiperbólico $\left(\mathbb{H}^{2}\right)$, enunciado adiante:

Teorema Considere, em $\mathbb{H}^{2}$, um quadrado com ângulo interno $\sigma$ e um círculo de mesma área, de raio $r$. Então, ambos são construtíveis se, e somente se, $0 \leq \sigma<\frac{\pi}{2}$ e $\sigma$ é um múltiplo inteiro de $\frac{2 \pi}{n}$, em que $n$ é um número inteiro positivo tal que o polígono regular de $n$ lados possa ser construído com régua e compasso no plano euclideano.

Tendo em vista que o resultado supracitado faz referência à construtibilidade de polígonos no plano euclideano, convém rememorar, a título elucidativo, o Teorema de Gauss-Wentzel:

Teorema Um polígono regular de $n$ lados pode ser construído com régua e compasso em $\mathbb{E}^{2}$ se, e somente se, $n$ é da forma

$$
2^{k} . F_{i_{1}} \cdot F_{i_{2}} \ldots F_{i_{r}}
$$

em que os $F_{i_{j}}$ são números primos de Fermat $\left(F_{l}=2^{2^{l}}+1\right)$.

Será apresentada uma demonstração do resultado supracitado, alicerçada no seguinte tripé:

(i) a observação de Bolyai de que o problema da construtibilidade do raio $r$ é equivalente à possibilidade de se construir um certo ângulo auxiliar $\theta$ (a prova desta equivalência é vista em detalhe);

(ii) o Teorema de Mordukhai-Boltovskoi, que estabelece a equivalência entre a construtibilidade de um segmento hiperbólico $\overline{P Q}$ e a construtibilidade, no sentido algébrico, das funções hiperbólicas de seu comprimento $d_{h}(P, Q)$;

(iii) o celebrado Teorema de Gelfond-Schneider, resposta ao sétimo problema de Hilbert sobre a irracionalidade e a transcendência de certos números.

Não se tentará apresentar nem mesmo esboços de provas para os dois últimos resultados: quanto ao primeiro, não julgamos que pudéssemos acrescentar nada de original àquela prova - elementar, mas um pouco longa - encontrada em [3], baseada ademais no modelo de Klein-Beltrami do plano hiperbólico, que não abordaremos nesta dissertação; já o segundo é um resultado profundo da Teoria dos Números, cuja demonstração escapa claramente aos objetivos colimados neste trabalho. Como se observa, também no caso hiperbólico, a resposta ao problema da quadratura do círculo passa por considerações atinentes a uma sofisticada Teoria dos Números. 
Serão enunciados e provados, ainda, dois teoremas adicionais, devidos a Jagy e expostos em [4], os quais estabelecem a inexistência de métodos de construção, no plano hiperbólico, que: 1) a partir do raio do círculo $r$, produzam o ângulo interno $\sigma$ do quadrado de mesma área e; 2) que, dado o ângulo interno do quadrado $\sigma$, permitam obter o raio $r$ do círculo de mesma área. Neste âmbito, serão apresentadas duas classes de exemplos de pares quadradocírculo de mesma área nas quais apenas uma das figuras (quadrado ou círculo) é construtível no plano hiperbólico.

Por consequência do exposto no parágrafo precedente, verifica-se que, na realidade, não se tem, no plano hiperbólico, uma "quadratura do círculo" nem uma "circulação do quadrado": as figuras devem ser construídas de forma simultânea ou independente. De todo modo, é relevante destacar que mesmo esta versão fraca do problema de construtibilidade é impossível na Geometria Euclideana, em virtude da transcendência de $\pi$.

No que diz respeito a pré-requisitos, foi feito um esforço para preservar este trabalho o máximo possível da introdução de conceitos e técnicas que envolvam um uso mais do que elementar do Cálculo Diferencial e Integral. Esta decisão foi devida tanto à presente inexperiência do autor com Geometria Diferencial, quanto à sua esperança de que estas notas sejam lidas por professores e estudantes dos últimos anos ou recém-egressos do nível secundário, e possam vir a motivar discussões entre eles a respeito de "outras geometrias", contribuindo para enriquecer a cultura matemática de educandos e educadores, no espírito do que propõe o ProfMat.

Após tais considerações preliminares, passaremos a apresentar os tópicos tratados nesta dissertação.

No Capítulo 2 (Geometria Euclideana), serão expostas as noções da Geometria Tradicional relacionadas ao posterior desenvolvimento do trabalho, sob a perspectiva de considerar conhecidos os principais fatos e os resultados mais básicos de tal Geometria. Após a exposição dos axiomas e postulados de Euclides, serão introduzidos, na seção 2.1, as regras do jogo de construção com régua e compasso, com apresentação de construções que serão utilizadas em outras partes do texto. No final do referido tópico serão relembrados os três problemas clássicos de construtibilidade: a quadratura do círculo, a duplicação do cubo e a trissecção do ângulo.

Na seção 2.2, a Geometria Analítica será apresentada como modelo para a Geometria Euclideana, com as provas de que as definições de reta como conjunto-solução de uma equação do primeiro grau $a x+b y=c(a, b, c \in \mathbb{R}, a$ e $b$ não simultaneamente nulos) e de círculo como conjunto-solução de uma equação da forma $\left(x-x_{C}\right)^{2}+\left(y-y_{C}\right)^{2}=r^{2}\left(x_{C}, y_{C}\right.$ e $r$ são números 
reais e $r>0$ ) satisfazem os postulados P1 a P5 de Euclides. No final deste tópico, será demonstrado um teorema, devido a Descartes, que relaciona a possibilidade de se construir um ponto, com régua e compasso, no plano cartesiano, com obtenção das suas coordenadas, a partir dos pontos iniciais, por meio das operações,,$+- \times, \div$ e da extração de raízes quadradas, o qual será fundamental no contexto desta dissertação quando do estudo da construtibilidade no plano hiperbólico.

Na seção 2.3, serão desenvolvidos os aspectos algébricos que conduziram à resposta negativa aos problemas clássicos de construtibilidade no plano euclideano (duplicação do cubo, trissecção do ângulo e quadratura do círculo).

A última seção do Capítulo 1 (2.4) será dedicada ao estudo da inversão circular, com prova das suas principais propriedades, as quais estão diretamente relacionadas com o desenvolvimento do modelo do disco de Poincaré, que será visto no Capítulo 3.

No capítulo 3 (Geometria Hiperbólica), após uma notícia histórica bem sucinta e da introdução do postulado 5 modificado, serão apresentadas as definições e os principais resultados da Geometria Hiperbólica que terão utilidade futura para a construção do quadrado e do círculo de mesma área no modelo do disco de Poincaré ou para a conclusão sobre as condições necessárias e suficientes para que seja possível tal construção.

A Geometria Hiperbólica será apresentada, no referido Capítulo (seção 3.1), com base no modelo do disco de Poincaré, que atribui novo significado a figuras já conhecidas da Geometria Euclideana: (i) o plano hiperbólico é representado pelo interior de um círculo e a circunferência deste círculo, chamada de absoluta, representa o infinito, o horizonte. Os pontos da absoluta são denominados pontos ideais; (ii) uma reta é um arco de círculo perpendicular à absoluta; (iii) duas retas são paralelas quando se cortam em um ponto ideal; (iv) as demais retas que não se intersectam são chamadas não-secantes ou divergentes.

Na subseção 3.1.1, será demonstrado que as definições da Geometria Hiperbólica satisfazem os postulados P1 a P4, além do P5 modificado. Será apresentada a construção euclideana da reta hiperbólica que passa por dois pontos dados, decorrente de uma das propriedades da inversão circular, vista no Capítulo 1. Serão enunciadas como teoremas e provadas as propriedades que permitem realizar a construção euclideana do círculo no modelo do disco de Poincaré. As conclusões de tal subseção são as de que a régua e o compasso, no modelo do disco de Poincaré, podem ser pensados como combinações ou usos mais sofisticados da régua e do compasso euclideanos e, ainda, de que 
a consistência da Geometria Hiperbólica depende apenas da consistência da Geometria Euclideana.

Na subseção 3.1.2, será retomada a definição de distância já mencionada na subseção anterior para introduzir a construção do círculo. Em seguida, serão apresentadas as definições de ângulo hiperbólico, ângulo de paralelismo e triângulo hiperbólico. A definição de polígono hiperbólico foi considerada conhecida, tendo em vista que é a mesma da Geometria Tradicional.

Ainda, na subseção 3.1.2, serão introduzidas as definições de defeito de um triângulo e defeito de um polígono - que consistem na diferença entre a soma dos ângulos internos que tais figuras teriam na Geometria Euclideana e a que efetivamente têm na Geometria Hiperbólica - e serão apresentadas provas de que todo triângulo tem defeito positivo e, por consequência, todo polígono também tem defeito positivo. Será provado, ainda, o fato de que, se dois triângulos são semelhantes na Geometria Hiperbólica, então eles são congruentes.

A área de um triângulo será definida, na subseção 3.1.2, como sendo igual ao seu defeito (como se verifica facilmente a partir do Teorema de Gauss-Bonnet, conforme se observa em [5]). Ofereceremos para tal fato uma fundamentação meramente heurística: partiremos do pressuposto de que a área deve ser um número positivo associado a um triângulo, com as características de invariância por congruência e aditividade, ambas satisfeitas pelo defeito. Por conseguinte, a área do polígono será dada também pelo seu defeito. Uma fórmula para a área do círculo será obtida pelo método da exaustão apenas na subseção seguinte, pois sua dedução dependerá de alguma trigonometria hiperbólica.

Na subseção 3.1.3, serão enunciadas e demonstradas as fórmulas da trigonometria hiperbólica para um triângulo retângulo hiperbólico e, em seguida, para um triângulo qualquer (Lei dos Senos e Lei dos Cossenos). Será demonstrada, ainda, a fórmula de Lobatchevski-Bolyai, que relaciona o comprimento de paralelismo $d$ com o respectivo ângulo $\alpha\left(e^{-d}=\tan \frac{\alpha}{2}\right)$. Serão provadas, também, outras fórmulas que relacionam ângulo e comprimento de paralelismo.

Na mesma subseção, será justificada, com base na fórmula de Lobatchevski-Bolyai, a existência de uma bijeção decrescente $\Pi: \mathbb{R}_{+} \backslash\{0\} \rightarrow$ $\left(0, \frac{\pi}{2}\right)$ que associa a cada comprimento $t$ o corresponde ângulo de paralelismo $\Pi(t)$. Ainda, será explicitada a existência de uma unidade natural de medida, oriunda da própria teoria da Geometria Hiperbólica, dada pela constante de Schweikart $(p)$, que corresponde à medida altura relativa à hipotenusa de um triângulo retângulo hiperbólico com lados infinitos e ao comprimento de 
paralelismo associado ao ângulo de $\frac{\pi}{4}$. Interessante observar que, na Geometria Euclideana, as unidades de medida são consideradas de forma arbitrária.

Ao final da subseção 3.1.3, será provada uma fórmula para a área do círculo, pelo método da exaustão, com utilização de uma proposição trigonométrica e de conceitos básicos de Cálculo Diferencial e Integral, como as séries de Taylor referentes às funções trigonométricas envolvidas na dedução da fórmula.

Na subseção 3.1.4 será ressaltado, inicialmente, que a noção de construtibilidade também faz sentido na Geometria Hiperbólica, mas, tendo em vista que o modelo do disco de Poincaré atribui novo significado a figuras já conhecidas da Geometria Euclideana, o leitor será advertido para que não confunda a noção de construbilidade euclideana em tal modelo com a noção de construtibilidade hiperbólica propriamente dita. Por esta razão, serão introduzidas as definições de ponto E-construtível e ponto H-construtível.

Ainda na subseção 3.1.4, serão retomados alguns dos tópicos sobre construtibilidade já discutidos no Capítulo 2 para então introduzir o já mencionado Teorema de Mordukhai-Boltovskoi, o qual estabelece que um comprimento $t$ pode ser construído com régua e compasso no plano hiperbólico se, e somente se, os comprimentos relativos às funções trigonométricas hiperbólicas de $t$ são construtíveis no plano euclideano. Com base no resultado mencionado, será provado um outro teorema, que será determinante para a introdução do ângulo auxiliar $\theta$ na análise do problema da quadratura do círculo em $\mathbb{H}^{2}$ : um ângulo $\alpha$ é construtível no plano hiperbólico se, e somente se, ele pode ser construído com régua e compasso no plano euclideano.

Sugere-se ao leitor já plenamente familiarizado com os pré-requisitos relativos à Geometria Hiperbólica que não se detenha por muito tempo na leitura dos Capítulos 1, 2 e 3 e avance diretamente para o Capítulo 4, no qual será efetivamente estudado o problema que é o objeto da presente dissertação.

Chegaremos, então, ao Capítulo 4, que diz respeito à Quadratura do Círculo no Plano Hiperbólico. Na seção 4.1, será apresentada, em detalhes, a construção do quadrado e do círculo de mesma área no modelo do disco de Poincaré, mas com a utilização da régua e compasso euclideanos, e tirando proveito do plano euclideano ambiente. Cabe insistir neste ponto, porque a construção não utilizará os métodos do Capítulo 3 e será inteiramente euclideana, extrínseca ao modelo, pois, como será visto, o círculo que representará o disco de Poincaré será construído juntamente com o restante da figura. As propriedades que justificam a construção serão provadas em proposições separadas. Será observado que os resultados da subseção 3.1.4 sugerem a existência 
de um método de construção intrínseco, embora não forneçam uma receita explícita.

Um último comentário sobre a construção supracitada: em analogia com o que ocorre na Geometria Euclideana, em que algumas construções não podem ser realizadas apenas com a utilização de régua e compasso, mas podem ser realizadas com o uso conjugado de outros instrumentos — como é o caso da duplicação do cubo com a utilização de um instrumento que permite traçar seções cônicas e as construções possíveis com régua graduada -, podemos pensar na construção da seção 4.1 como intrínseca, mas utilizando dois instrumentos (hipotéticos): um que desenha segmentos de retas euclideanas e outro que permite traçar arcos de círculos euclideanos secantes ao disco de Poincaré, seja lá os nomes e a aparência que essas curvas tenham para um (hipotético?) habitante do espaço hiperbólico $\mathbb{H}^{3}$.

Ainda na seção 4.1 e após a construção, serão apresentados questionamentos sobre a possibilidade de se construir um quadrado e um círculo de mesma área no plano hiperbólico. Será demonstrada, também, uma fórmula que relaciona o raio euclideano do círculo hiperbólico $(x)$ com o ângulo interno $\sigma$ do quadrado; em seguida, serão apresentados três exemplos, que são $\sigma=\frac{\pi}{4}$, $r=\ln \left(\frac{16+5 \sqrt{7}}{9}\right)$ e $\sigma=\arctan \frac{3}{4}$, em que $r$ é o raio hiperbólico do círculo, com o objetivo de fixar ideias e possibilitar a conclusão sobre o resultado que será visto na sequência.

Finalmente, na seção 4.2, será enunciado o Teorema de Nestorovich-Jagy, que estabelece as condições necessárias e suficientes para que um quadrado e um círculo de mesma área sejam ambos construtíveis no plano hiperbólico, conforme já detalhado anteriormente, com prova baseada no tripé já apontado acima. Ao contrário do desenvolvido no Capítulo 3, o tratamento do tema nesta última seção será feito do ponto de vista sintético. Assim, serão expostas, antes, as construções necessárias à obtenção do quadrado hiperbólico e à prova do fato, observado por Bolyai, de que o problema da construbilidade do raio $r$ do círculo equivale ao da construtibilidade do ângulo auxiliar $\theta$, tal que $\tan \theta=2 \sinh \frac{r}{2}$.

Como já explicitado, serão provados, ainda, dois teoremas devidos a Jagy, expostos em [4], que estabelecem a inexistência de métodos de construção com régua e compasso, no plano hiperbólico, para, a partir do quadrado, obter o círculo de mesma área e vice-versa. Para provar o último destes resultados, será introduzido o Teorema de Olmsted, que consta de [6], nos termos do qual se o ângulo $\tau$ é um múltiplo racional de $\pi$, então os únicos valores racionais para $\tan \tau$ são 0,1 e -1 . 


\section{2}

\section{Geometria Euclideana}

A Geometria Tradicional ou Euclideana foi desenvolvida por Euclides em sua monumental obra "Elementos", que continha tópicos de diversas áreas da Matemática até então conhecida. Este matemático grego estabeleceu um conjunto de axiomas e postulados. Os axiomas são os seguintes:

AXIOMA 1. Coisas iguais a uma terceira também são iguais entre si.

AXIOMA 2. Se quantidades iguais são adicionadas a partes iguais, os resultados serão iguais.

AXIOMA 3. Se quantidades iguais são subtraídas de coisas iguais, os restos são iguais.

AXIOMA 4. Coisas que coincidem umas com as outras são iguais.

AXIOMA 5. O todo é maior que suas partes.

Já os postulados, que dizem respeito, especificamente, à Geometria, são enunciados adiante:

$\mathbf{P 1}$. Uma reta pode ser traçada de um ponto a outro, escolhidos à vontade.

P2. Uma reta pode ser prolongada indefinidamente.

P3. Um círculo pode ser traçado com centro e raio arbitrários.

$\mathbf{P} 4$. Todos os ângulos retos são iguais (congruentes).

P5. Se uma reta é transversal a outras duas e forma com estas ângulos, de um mesmo lado da transversal, que somam menos de dois ângulos retos, então as duas retas dadas, desde que prolongadas de forma suficiente, intersectar-seão em um ponto deste mesmo lado.

O postulado P5 é mais conhecido pela sua forma moderna, enunciada a seguir:

P5(Enunciado Moderno - Axioma de Playfair). Por um ponto do plano exterior a uma reta é possivel traçar uma única reta paralela à reta dada.

Como o presente trabalho diz respeito a um problema de construtibilidade, é pertinente destacar que os postulados P1 e P3, acima enunciados, podem ser traduzidos como "existem réguas" e "existem compassos". Assim, os problemas de construção com régua e compasso dizem respeito a saber se uma dada figura pode ser construída por meio de retas, círculos ou arcos de círculos e a interseção destes (duas retas, círculos e retas, dois círculos). 
Nesta dissertação, serão considerados conhecidos os principais fatos e os resultados mais básicos da Geometria Euclideana, os quais serão utilizados sem uma exposição sistemática anterior.

\section{1}

\section{Construtibilidade com Régua e Compasso}

\subsection{1}

\section{Noções Gerais}

Conforme visto na seção anterior, os postulados P1, P2 e P3 estabelecem a existência de retas e círculos no plano euclideano, e, por via de consequência, permitem imaginar a existência instrumentos que possibilitem traçar tais figuras. A régua é o instrumento que permite desenhar retas e o compasso é o instrumento permite a construção de círculos.

Quando se pensa em problemas de construtibilidade com régua e compasso, não se cogita da existência ideal das figuras geométricas, mas sim da possibilidade de se realizar a sua construção com a utilização de um método exato e não aproximado.

Os problemas de construtibilidade devem ser resolvidos com régua não marcada, ou seja, que não contém unidades de medida. Ademais, deve ser utilizado o compasso colapsante, ou seja, aquele que permite diversas aberturas para obter raios de círculos arbitrários, mas, se uma das pontas for retirada do papel, não é possível manter a abertura inicialmente considerada, de modo que não é possível transferir as medidas diretamente.

Outra observação importante é que, nas construções realizadas, não são considerados pontos arbitrários nem aberturas de compasso quaisquer; todos os pontos e medidas de abertura utilizados nas construções têm de ser previamente dados.

Ainda que haja as restrições mencionadas no parágrafo anterior, é possível estabelecer, com os referidos instrumentos, um método de construção para realizar a transferência de medidas. É o que veremos adiante.

Proposição 1 (Transferência de Medidas) É possivel transferir medidas com régua não marcada e compasso colapsante.

Prova. Para provarmos a proposição acima, realizaremos uma construção para a transferência de medidas proposta no enunciado acima.

Sejam dados um segmento $\overline{A B}$ e o ponto $O$, a partir do qual será transferida a medida de $\overline{A B}$. A construção, conforme a figura acima, tem as seguintes etapas: 


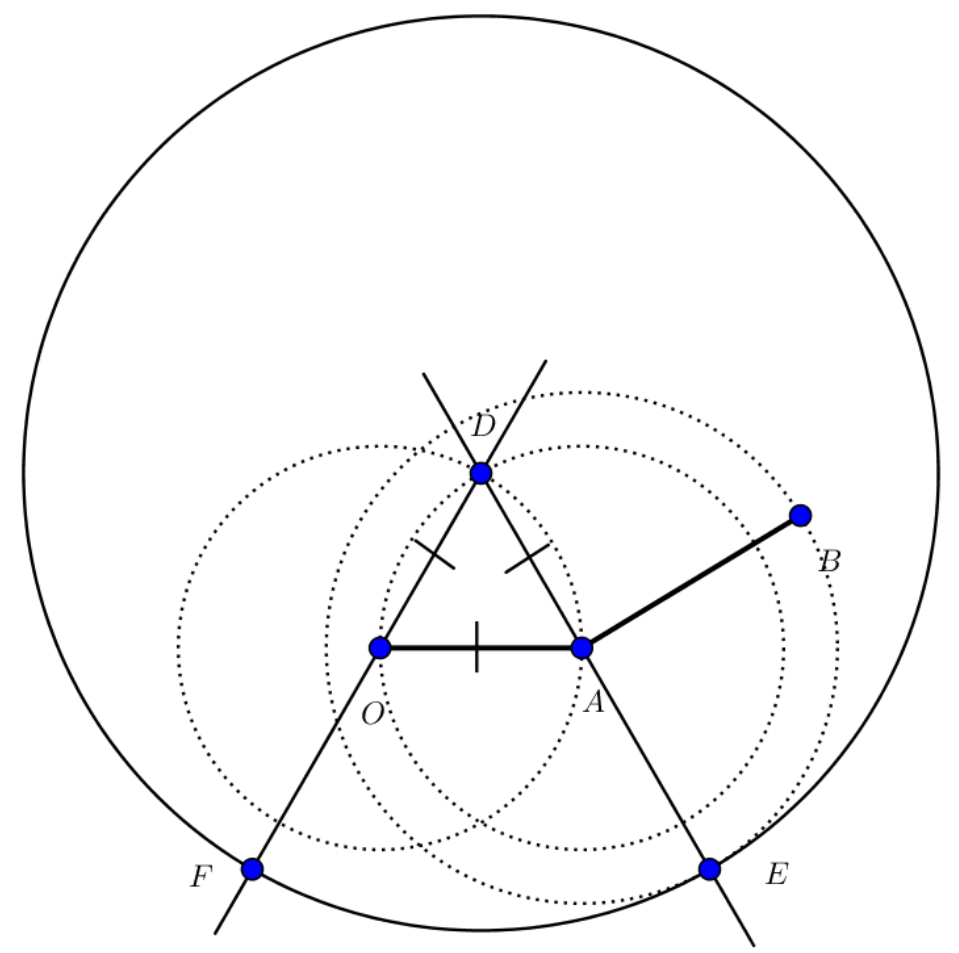

Figura 2.1: Construção - Transferência de Medida de Segmento

1 - traçar os círculos de centro $A$ e raio $\overline{A O}$ e de centro $O$ e mesmo raio. As circunferências terão uma das suas interseções no ponto $D$ (figura 2.1);

2 - desenhar o círculo de centro $A$ e raio $\overline{A B}$ e a reta $\overleftrightarrow{D A}$, prolongando-a do modo suficiente até que encontre esta última circunferência no ponto $E$, do lado oposto de $D$ em relação a $A$;

3 - traçar o círculo de centro $D$ e raio $\overline{D E}$ e, em seguida, desenhar a reta $\overleftrightarrow{D O}$, que encontrará a circunferência centrada em $D$ no ponto $F$, no lado oposto de $D$ em relação a $O$. O segmento $\overline{O F}$ tem a mesma medida de $\overline{A B}$.

Para justificar a construção acima e finalizar a demonstração, basta observar que o triângulo $A O D$ é equilátero e se tem $\overline{A O}=\overline{D A}=\overline{D O}$. Ademais, como $B$ e $E$ estão sobre o mesmo círculo centrado em $A$, tem-se que $\overline{A B}=\overline{A E}$. Uma vez que $E$ e $F$ estão sobre o mesmo círculo centrado em $D$, chega-se a

$$
\overline{D F}=\overline{D E} \Rightarrow \overline{D O}+\overline{O F}=\overline{D A}+\overline{A E} \Rightarrow \overline{D O}+\overline{O F}=\overline{D A}+\overline{A B}
$$

Mas $\overline{D A}=\overline{D O}$, donde segue a igualdade $\overline{O F}=\overline{A B}$, o que prova a proposição enunciada acima.

É interessante observar que a construção vista acima não depende do quinto postulado de Euclides. Tal fato terá extrema relevância quando realizarmos o estudo da Geometria Hiperbólica. 
Na sequência, apresentaremos outras construções geométricas que terão importância no contexto deste trabalho. Algumas destas construções serão extremamente relevantes quando tratarmos do modelo do Disco de Poincaré e da construção do quadrado e do círculo de mesma área no contexto da Geometria Hiperbólica.

Construção 1 (Perpendicular por ponto exterior) Dada uma reta $r$, um ponto $A$ sobre $r$ e um ponto exterior $P$, construir a reta perpendicular a $r$ que passa por $P$.

A construção acima é realizada adotando-se os seguintes passos:

1 - utilizar o compasso para construir um círculo de centro $P$ e raio $\overline{A P}$, que intersectará $r$ em outro ponto $B$;

2 - traçar as circunferências de centros $A$ e $B$ e raios $\overline{A P}$ e $\overline{B P}$, que se cortarão nos pontos $P$ e $Q$;

3 - desenhar a reta $\overleftrightarrow{P Q}$, que é a perpendicular procurada.

Justificativa. Pela construção, tem-se que $\overline{A P}=\overline{B P}=\overline{A Q}=\overline{B Q}$, donde segue que os triângulos $A P B$ e $A Q B$ são isósceles e congruentes, do mesmo que os triângulos $P A Q$ e $P B Q$ são isósceles e congruentes.

Seja $H$ o ponto de interseção das retas $r$ e $\overleftrightarrow{P Q}$ (não mostrado na figura). Os triângulos $A P B$ e $A Q B$ são congruentes e os segmentos $\overline{P H}$ e $\overline{Q H}$, em cada um dos triângulos, são cevianas relativas ao lado comum $\overline{A B}$, as quais dividem este lado nos mesmos segmentos $\overline{A H}$ e $\overline{H B}$. Assim, $\overline{P H}=\overline{H Q}$.

Por conseguinte, como $A P Q$ é isósceles e $\overline{P H}=\overline{H Q}$, segue que $\overline{A H}$ é mediana relativa ao lado $\overline{P Q}$, que se confunde com a altura, donde se conclui que $r$ e $\overleftrightarrow{P Q}$ são perpendiculares.

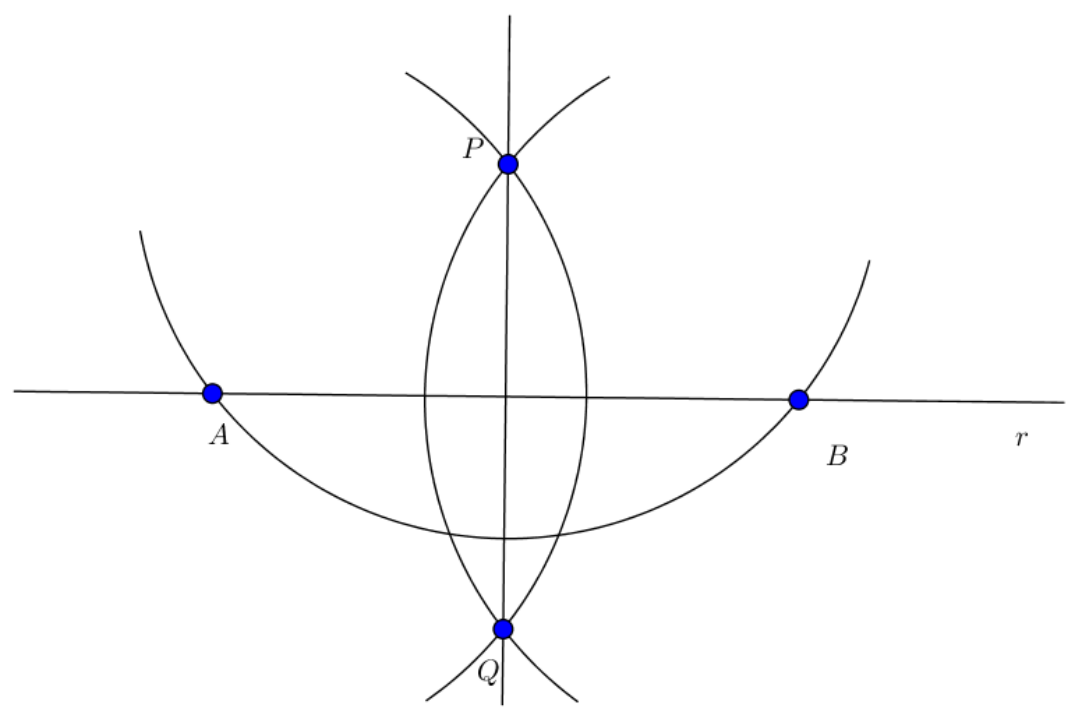

Figura 2.2: Perpendicular por ponto exterior à reta dada 
Construção 2 (Perpendicular por um ponto incidente) Sejam uma reta $r$ e dois pontos sobre esta $A$ e $P$. Traçar a reta perpendicular a $r$ que passa por $P$.

Tal construção é realizada da seguinte forma:

1 - usar o compasso para traçar a circunferência de centro $P$ e raio $\overline{P A}$, que intersectará $r$ em outro ponto $Q$;

2 - desenhar as circunferências com raio $\overline{A Q}$ e centros $A$ e $Q$, respectivamente. As referidas circunferências terão interseção nos pontos $B$ e $C$;

3 - traçar a reta $\overleftrightarrow{B C}$, que passará por $P$ e será a reta perpendicular procurada.

Justificativa. Da figura, tem-se que $\overline{A Q}=\overline{A B}=\overline{B Q}=\overline{A C}=\overline{C Q}$. Além disso, $\overline{A P}=\overline{P Q}$. Logo, os triângulos $A B Q$ e $A C Q$ são equiláteros e $P$ é o pé das medianas de tais triângulos $(\overline{B P}$ e $\overline{C P})$ relativas a $\overline{A Q}$, que também são alturas. Portanto, $\overleftrightarrow{B C}$ passa por $P$ e é perpendicular a $r$

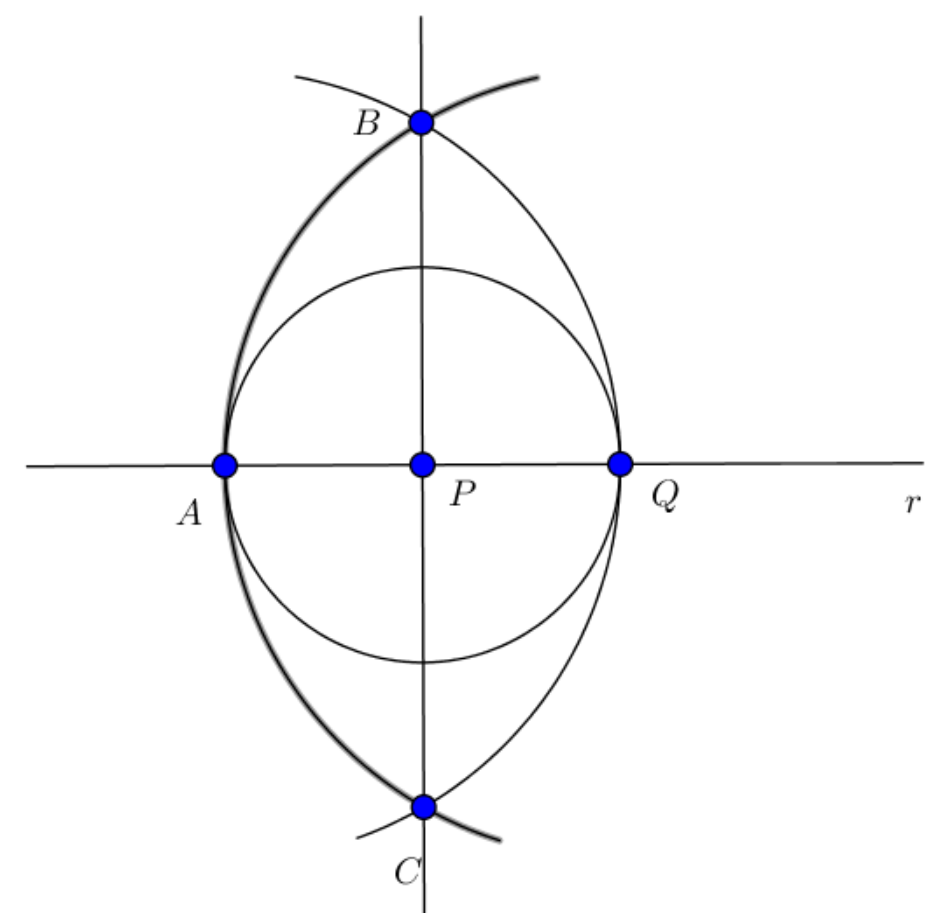

Figura 2.3: Perpendicular por um ponto incidente

É relevante destacar que, se fossem dados $A$ e $Q$, os passos 2 e 3 acima descritos permitiriam obter, por construção, a mediatriz do segmento $\overline{A Q}$.

Construção 3 (Paralela) Por um $P$ exterior a uma reta $r$, com ponto incidente $A$, construir uma reta s paralela a $r$.

Já sabemos construir retas perpendiculares. Deste modo, para construir a reta paralela procurada, podemos proceder da seguinte forma: 
1 - traçar a reta $t$, perpendicular a $r$ e passando por $P$;

2 - desenhar a reta $s$, perpendicular a $t$ e passando por $P$. A reta $s$ é a paralela procurada.

Justificativa. As retas $r$ e $s$ formam um ângulos que somam dois ângulos retos em quaisquer dos lados da transversal $t$. Assim, pelo quinto postulado de Euclides, não é possível que tais retas se intersectem em nenhum ponto, donde se conclui que $r$ e $s$ são paralelas.

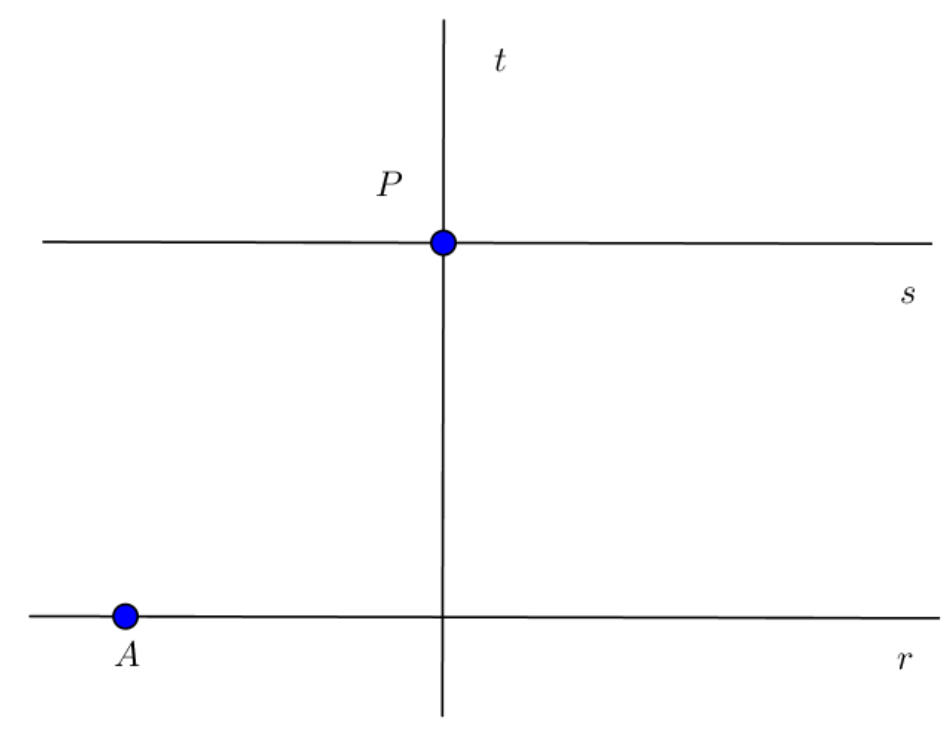

Figura 2.4: Retas paralelas

Construção 4 (Bissetriz) Dadas suas semirretas $O A$ e $O B$ de origem comum $O$, traçar a bissetriz do ângulo $A \hat{O} B$.

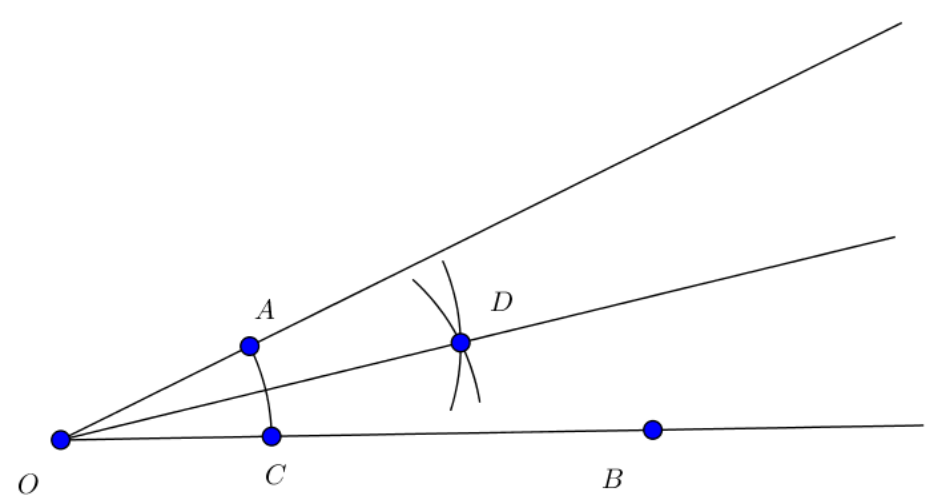

Figura 2.5: Construção da bissetriz

A fim de traçarmos a bissetriz do ângulo acima referido, devemos proceder da seguinte forma:

1 - desenhar a circunferência de centro $O$ e raio $\overline{O A}$, que intersectará a semirreta $O B$ no ponto $C$; 
2 - traçar as circunferências de centro $A$ e $C$, com raios de medida idêntica à do segmento $\overline{O A}$. Tais circunferências encontrar-se-ão no ponto $D$, no interior do ângulo $A \hat{O} B$;

3 - traçar a semirreta $\overrightarrow{O D}$, que é a bissetriz procurada.

Justificativa. Da figura acima, tem-se que $\overline{O A}=\overline{O C}=\overline{A D}=\overline{C D}$. Deste modo, os triângulos $O A D$ e $O C D$ são congruentes, donde segue, pela correspondência dos ângulos, que $A \hat{O} D=D \hat{O} C$. Assim, conclui-se que $O D$ é bissetriz de $A \hat{O} B$.

Importante observar que, na figura acima, a construção foi realizada como se o compasso fosse não colapsante, ou seja, como se este retivesse a abertura após uma das suas ter sido retirada do papel e pudesse transferir diretamente medidas. No entanto, tal fato não é realmente relevante, uma vez que foi provado acima que é possível transferir medidas mesmo com o compasso colapsante, de modo que poderia ser utilizado o método explicitado na prova da proposição 1 para realizar a transferência da medida de $\overline{O A}$.

Construção 5 (Tangentes) Traçar as retas tangentes a um círculo de centro

$O$, que passam por um ponto $A$, exterior ao círculo.

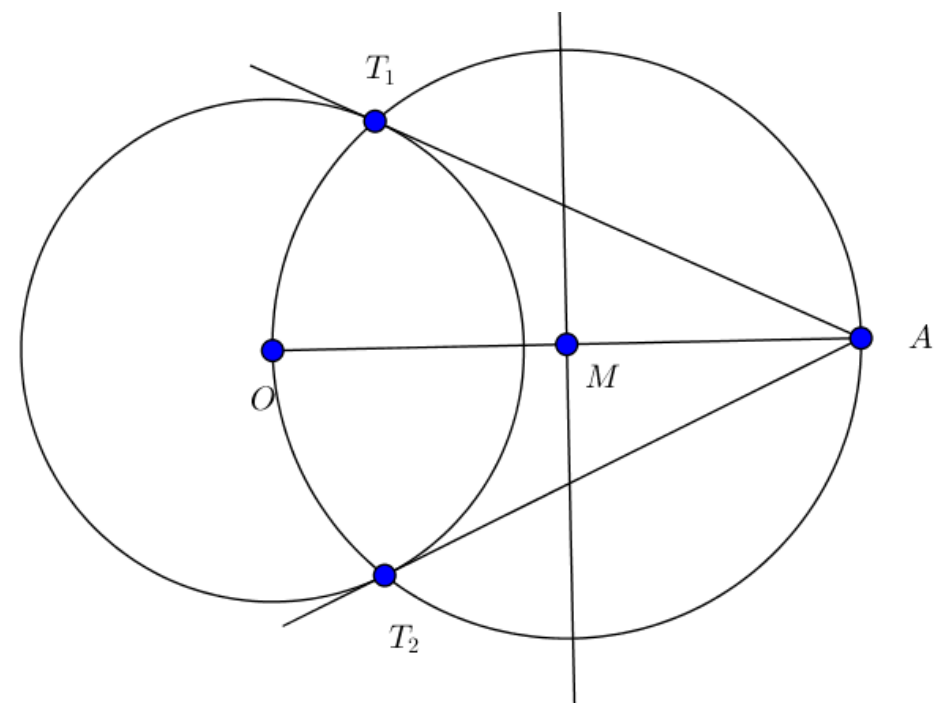

Figura 2.6: Retas tangentes por um ponto exterior ao círculo

Para traçar as retas tangentes a um círculo de centro $O$, que passam por um ponto exterior $A$, deve-se proceder da seguinte forma:

1 - traçar o segmento $\overline{O A}$;

2 - traçar a reta mediatriz do segmento $\overline{O A}$, obtendo o ponto médio $M$;

3 - desenhar a circunferência com centro $M$ e raio $\overline{O M}=\overline{M A}$, que intersectará o círculo original nos pontos $T_{1}$ e $T_{2}$; 
4 - traçar as retas $\overleftrightarrow{A T_{1}}$ e $\overleftrightarrow{A T_{2}}$, que são as tangentes procuradas.

Justificativa. Basta observar que, do ponto de vista da circunferência de centro $M$, o segmento $\overline{O A}$ é diâmetro. Deste modo, os triângulos $O T_{1} A$ e $O T_{2} A$ são retângulos em $T_{1}$ e em $T_{2}$, respectivamente. Daí, segue que os raios $\overline{O T_{1}}$ e $\overline{O T_{2}}$, do círculo dado, são perpendiculares às retas $\overleftrightarrow{A T_{1}}$ e a $\overleftrightarrow{A T_{2}}$, respectivamente, donde se conclui que estas retas são as tangentes procuradas.

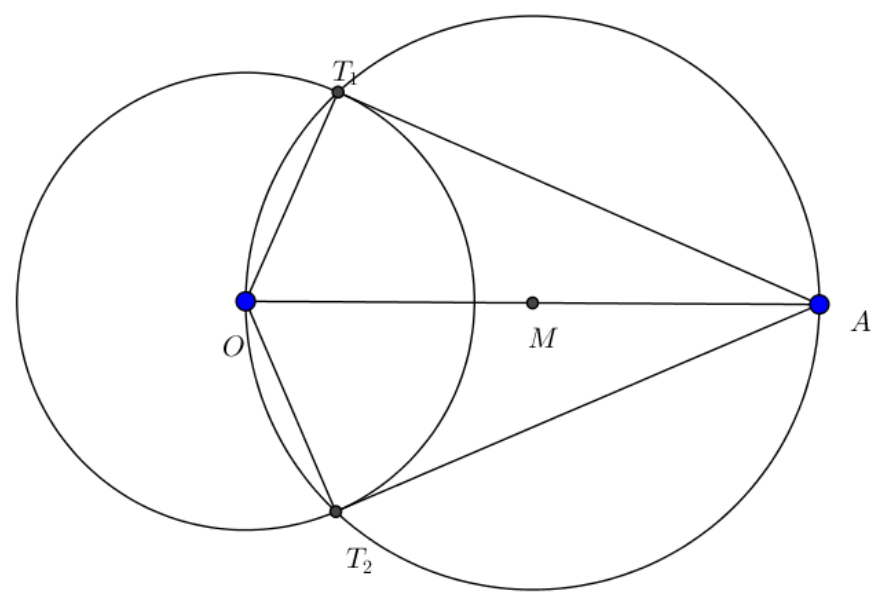

Figura 2.7: Justificativa para a construção das retas tangentes

\subsection{2}

\section{Três Problemas Clássicos}

Há três problemas clássicos de construtibilidade que foram cogitados ainda na Grécia Antiga. Neste contexto, é relevante expor um pouco da história relativa a tais problemas.

No livro [7], contam os autores, com base em Plutarco, que o filósofo pré-socrático Anaxágoras, falecido em 428 a. C., enquanto esteve preso, empreendeu tentativas de quadrar o círculo, ou seja, de construir, com régua e compasso, um quadrado que tivesse a mesma área de um círculo dado.

A obra supracitada menciona uma lenda associada ao segundo problema clássico apresentado: conta-se que uma peste vitimou Péricles em 427 a. C. e assolou Atenas, tendo ceifado, possivelmente, um quarto da população da cidade. Assim, uma delegação teria sido enviada ao Oráculo de Apolo em Delos, para indagar sobre como combater a doença; o Oráculo teria respondido que, para aplacar os efeitos da peste, o altar de Apolo, que tinha formato cúbico, deveria ser duplicado. Em vão tentaram os atenienses resolver o problema duplicando a aresta do cubo, já que este sólido teve seu volume multiplicado por oito.

Por último, narram os autores acima referidos que, por este mesmo período, circulava por Atenas um outro problema de construtibilidade, que 
dizia respeito à possibilidade de se construir, apenas com régua e compasso, um ângulo com medida equivalente a um terço da medida do ângulo dado.

Portanto, os três problemas clássicos são os seguintes:

Problema 1 (Quadratura do Círculo) Construir, com régua e compasso, um quadrado que tenha a mesma área que um círculo dado.

Problema 2 (Duplicação do Cubo) Construir, com régua e compasso, a aresta de um cubo que tenha volume correspondente a duas vezes o volume de um cubo dado.

Problema 3 (Trissecção do Ângulo) Construir, com régua e compasso, um ângulo com medida que corresponde a um terço da medida de um ângulo dado.

Como já se sabe, os três problemas clássicos são, em geral, impossíveis de serem resolvidos na Geometria Euclideana, mas foram necessários mais de 2200 anos e o auxílio de Álgebra Moderna para que a resposta negativa aos problemas fosse dada.

Mais adiante no texto, serão explicitados os motivos da impossibilidade de solução para os referidos problemas no contexto da Geometria Tradicional.

\section{2}

\section{Geometria Analítica}

Nesta seção, apresentaremos algumas questões gerais sobre a Geometria Analítica, a qual terá alguma relevância no desenvolvimento do tema trabalhado nesta dissertação.

Do ponto de vista analítico, um ponto é um conjunto unitário contendo um par ordenado $(x, y)$ de números reais, ou seja, um ponto $P$ é da forma $\{(x, y)\}$. O plano euclideano é o conjunto $\mathbb{R}^{2}=\{(x, y), x, y \in \mathbb{R}\}$. A reta é definida da forma a seguir:

Definição 1 (Reta) Uma reta, do ponto de vista analítico, é o conjuntosolução de uma equação do primeiro grau $(a x+b y=c)$, em que são $a, b$, c são coeficientes reais e a e b não podem ser simultaneamente nulos. Ou seja, a reta $r$ é dada por $r=\left\{(x, y) \in \mathbb{R}^{2} \mid a x+b y=c\right\}$.

Existem duas retas especialmente importantes, $x=0$ e $y=0$, que passam pela origem $O=\{(0,0)\}$ e permitem estabelecer um sistema de coordenadas ortogonais para os pontos do plano. A reta $y=0$, neste contexto, é chamada de eixo das abscissas e a reta $x=0$ é chamada eixo das ordenadas. 


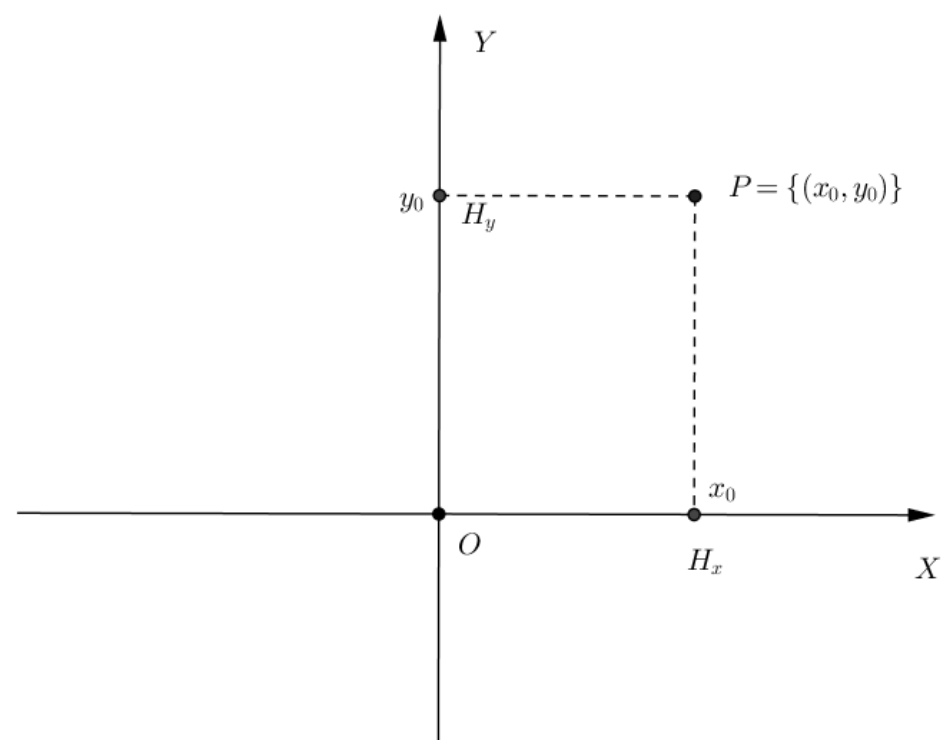

Figura 2.8: Eixos coordenados

Dado um ponto $P\left\{\left(x_{0}, y_{0}\right)\right\}$, o número $x_{0}$ é a coordenada do pé da perpendicular $\overline{P H_{x}}$ ao eixo das abscissas, isto é, a medida do segmento $\overline{O H_{x}}$, com sinal positivo se $H_{x}$ estiver à direita de $O$ e negativo em caso contrário.

Analogamente, $y_{0}$ é a coordenada do pé da perpendicular $\overline{P H_{y}}$ ao eixo das ordenadas (medida do segmento $\overline{O H_{y}}$ ), com sinal positivo se $H_{y}$ está acima de $O$ ou negativo, se $H_{y}$ estiver abaixo de $O$.

No contexto da Geometria Analítica, existe uma definição de distância (métrica), que será introduzida a seguir.

Definição 2 (Distância entre Pontos) Sejam $P=\left\{\left(x_{P}, y_{P}\right)\right\}$ e $Q=$ $\left\{\left(x_{Q}, y_{Q}\right)\right\}$ dois pontos no plano euclideano. A distância entre os dois pontos é definida por

$$
|\overline{P Q}|=\sqrt{\left(x_{P}-x_{Q}\right)^{2}+\left(y_{P}-y_{Q}\right)^{2}}
$$

Convém destacar que a distância entre pontos acima definida tem a seguinte propriedades:

(i) Positividade. $|\overline{P Q}| \geq 0$. Note-se que $\overline{P Q}=0 \Leftrightarrow P=Q$;

(ii) Simetria. $|\overline{P Q}=| \overline{Q P} \mid$;

(iii) Desigualdade Triangular. $|\overline{P Q}| \leq|\overline{P R}|+|\overline{R Q}|$.

Com a definição de distância acima exposta, estamos em condições definir o círculo do ponto de vista analítico.

Definição 3 (Círculo) Um círculo $\Gamma$, do ponto de vista analítico, é o conjunto-solução de uma equação da forma $\left(x-x_{C}\right)^{2}+\left(y-y_{C}\right)^{2}=r^{2}$ em que $x_{C}, y_{C}$ e $r>0$ são constantes reais. Ou seja, $\Gamma=\left\{(x, y) \in \mathbb{R}^{2} \mid\left(x-x_{C}\right)^{2}+\right.$ $\left.\left(y-y_{C}\right)^{2}=r^{2}, r>0\right\}$. 
Observe-se que, considerando a definição de distância acima exposta, a equação que representa o círculo corresponde à sua definição axiomática de conjunto dos pontos do plano que estão a uma certa distância (raio) de um ponto dado (centro), pois o ponto $C=\left\{\left(x_{C}, y_{C}\right)\right\}$ é o seu centro e $r$ é o raio.

Definição 4 (Incidência) Dizemos que um objeto $A$ (ponto, reta ou círculo) incide num objeto $B$ (ponto, reta ou círculo) se, e somente se, $A \cap B \neq \emptyset$.

As definições de reta e círculo acima satisfazem os postulados P1 a P5 de Euclides. É o que provaremos a seguir.

Proposição 2 A definição 1 (reta) satisfaz o postulado 1 de Euclides.

Prova. Para provarmos a proposição acima, devemos mostrar, primeiramente, que, dados dois pontos $P=\left\{\left(x_{P}, y_{P}\right)\right\}$ e $Q=\left\{\left(x_{Q}, y_{Q}\right)\right\}$, existe uma reta passando por eles. Seja $r$ a reta de equação $\left(y_{Q}-y_{P}\right) \cdot x-\left(x_{Q}-x_{P}\right) \cdot y=$ $x_{P} y_{Q}-x_{Q} y_{P}$. De fato, $P$ incide em $r$, pois:

$\left(y_{Q}-y_{P}\right) \cdot x_{P}-\left(x_{Q}-x_{P}\right) \cdot y_{P}=y_{Q} x_{P}-y_{P} x_{P}-x_{Q} y_{P}+x_{P} y_{P}=x_{P} y_{Q}-x_{Q} y_{P}$

ou seja, a equação de $r$ é verificada por $P$. Do mesmo modo, $r$ passa por $Q$, pois:

$\left(y_{Q}-y_{P}\right) \cdot x_{Q}-\left(x_{Q}-x_{P}\right) \cdot y_{Q}=y_{Q} x_{Q}-y_{P} x_{Q}-x_{Q} y_{Q}+x_{P} y_{Q}=x_{P} y_{Q}-x_{Q} y_{P}$

Provaremos, agora, que a reta $r$, que passa pelos pontos $P$ e $Q$, é única. Suponhamos que exista outra reta $(s)$, de equação $a x+b y=c(\mathrm{I})$, que passe por $P$ e $Q$. Deste modo, temos que $a x_{P}+b y_{P}=c$ (II) e $a x_{Q}+b y_{Q}=c$ (III). Subtraindo (II) de (III), obtemos:

$a\left(x_{Q}-x_{P}\right)+b\left(y_{Q}-y_{P}\right)=0 \Rightarrow a\left(x_{Q}-x_{P}\right)=-b\left(y_{Q}-y_{P}\right) \Rightarrow-\frac{a}{b}=\frac{y_{Q}-y_{P}}{x_{Q}-x_{P}}$

o que é possível, pois $a$ e $b$ não podem ser simultaneamente nulos (foi suposto $b$ não nulo). Daí, segue que $a=-\lambda\left(y_{Q}-y_{P}\right)(\mathrm{IV})$ e $b=\lambda\left(x_{Q}-x_{P}\right)(\mathrm{V})$, com $\lambda \in \mathbb{R}, \lambda \neq 0$. Além disto, como $P$ incide em $s$, conclui-se que $c=a x_{P}+b y_{P}$ (VI). Substituindo (IV), (V) e (VI) em (I), obtemos:

$$
-\lambda\left(y_{Q}-y_{P}\right) x+\lambda\left(x_{Q}-x_{P}\right) y=-\lambda\left(y_{Q}-y_{P}\right) x_{P}+\lambda\left(x_{Q}-x_{P}\right) y_{P}
$$

Dividindo a equação acima por $-\lambda$ e realizando as operações necessárias, chegamos a $\left(y_{Q}-y_{P}\right) x-\left(x_{Q}-x_{P}\right) y=x_{P} y_{Q}-x_{Q} y_{P}$, que é a equação de $r$ (contradição). Logo, existe apenas uma reta que passa por $P$ e $Q$. 
Proposição 3 A definição 1 (reta) satisfaz o postulado 2 de Euclides.

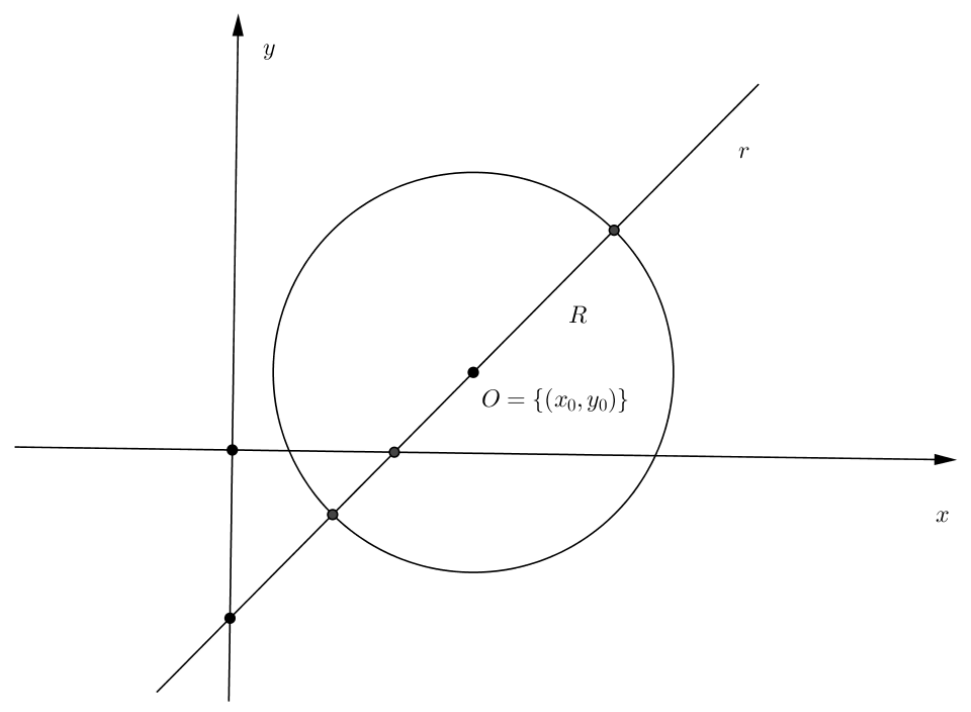

Figura 2.9: Ilustração - reta prolongada indefinidamente

Prova. Para mostrar que a definição analítica de reta satisfaz o postulado 2 uma reta pode ser prolongada indefinidamente - , podemos pensar da seguinte forma: dada uma reta $r$ e um círculo $\Gamma$, com diâmetro sobre $r$, devemos mostrar que existem pontos de $r$ que não estão não no interior de $\Gamma$.

Seja $r$ um reta de equação $a x+b y=c$ e seja $O=\left\{\left(x_{0}, y_{0}\right)\right\}$ o centro do círculo $\Gamma$, que tem raio $R$. Assim, a equação do círculo é dada por $\left(x-x_{0}\right)^{2}+\left(y-y_{0}\right)^{2}=$ $R^{2}$. Como $O$ incide em $r$ por hipótese, segue que $c=a x_{0}+b y_{0}$. Portanto, a equação de $r$ pode ser reescrita como $a x+b y=a x_{0}+b y_{0}$.

Mostraremos que existem dois pontos sobre $r$ que estão a uma distância $2 R$ de $O$ e, portanto, não estão no interior do círculo $\Gamma$. Para tanto, resolveremos o sistema dado pelas equações $a x+b y=a x_{0}+b y_{0}(\mathrm{I})$ e $\left(x-x_{0}\right)^{2}+\left(y-y_{0}\right)^{2}=4 R^{2}$ (II).

Fazendo as operações necessárias em (I), obtemos $x-x_{0}=-\frac{b}{a} \cdot\left(y-y_{0}\right)$. Substituindo $x-x_{0}=z$ e $y-y_{0}=w$, chegamos ao sistema formado pelas equações $z=-\frac{b}{a} \cdot w\left(\right.$ III) e $z^{2}+w^{2}=4 R^{2}$ (IV).

Substituindo (III) em (IV), obtemos

$$
\frac{b^{2}}{a^{2}} \cdot w^{2}+w^{2}=4 R^{2} \Rightarrow\left(a^{2}+b^{2}\right) \cdot w^{2}=4 a^{2} R^{2} \Rightarrow w= \pm \frac{2 a R}{\sqrt{a^{2}+b^{2}}}
$$

Substituindo o valor encontrado para $w$ em (III), chegamos a

$$
z=\mp \frac{2 b R}{\sqrt{a^{2}+b^{2}}}
$$


. Por fim, obtemos os pontos

$$
\left\{\left(-\frac{2 b R}{\sqrt{a^{2}+b^{2}}}+x_{0}, \frac{2 a R}{\sqrt{a^{2}+b^{2}}}+y_{0}\right)\right\}
$$

$\mathrm{e}$

$$
\left\{\left(\frac{2 b R}{\sqrt{a^{2}+b^{2}}}+x_{0},-\frac{2 a R}{\sqrt{a^{2}+b^{2}}}+y_{0}\right)\right\},
$$

que pertencem à reta $r$ mas não estão no interior de $\Gamma$. Assim, concluímos que a reta, com a definição analítica dada acima, pode ser prolongada indefinidamente.

Proposição 4 A definição 3 (círculo) satisfaz o postulado 3 de Euclides.

Prova. Pela definição 3, um círculo é o conjunto dos pontos do $\mathbb{R}^{2}$ que satisfazem equações da forma $\left(x-x_{c}\right)^{2}+\left(y-y_{c}\right)^{2}=R^{2}$, em que $C=\left\{\left(x_{c}, y_{c}\right)\right\}$ é o centro do círculo e $R>0$ é o raio. Deste modo, variando os parâmetros do ponto $C$ e a medida do raio $R$, vemos claramente, que é possível traçar um círculo com centro e raio considerados arbitrariamente.

Proposição 5 As definições da Geometria Analítica satisfazem o postulado 4 de Euclides, segundo o qual todos os ângulos retos são iguais (congruentes).

Prova. Para demonstrar que as definições da Geometria Analítica satisfazem o postulado 4, devemos mostrar que, dados dois pares retas perpendiculares no plano, é possível obter uma transformação - uma combinação de rotação e translação - que leva um par de retas no outro.

Sejam $r_{1}$ e $s_{1}$ duas retas perpendiculares com interseção no ponto $\left(a_{1}, b_{1}\right)$, tal que $r_{1}$ forma um ângulo $\theta_{1}$ com o eixo $x$. Considere, ainda, as retas perpendiculares $r_{2}$ e $s_{2}$, com interseção no ponto $\left(a_{2}, b_{2}\right)$, tal que $r_{2}$ forma um ângulo $\theta_{2}$ com o eixo das abscissas. As transformações que levam as referidas perpendiculares nos eixos $x$ e $y$ é dada por

$$
T_{j}\left(\begin{array}{l}
x \\
y
\end{array}\right)=R_{\theta_{j}}\left(\begin{array}{l}
x \\
y
\end{array}\right)-\left(\begin{array}{l}
a_{j} \\
b_{j}
\end{array}\right), \quad \text { onde } \quad R_{\theta_{j}}=\left(\begin{array}{rr}
\cos \theta_{j} & -\sin \theta_{j} \\
\sin \theta_{j} & \cos \theta_{j}
\end{array}\right) .
$$

Queremos uma transformação $T$ que leve $r_{1}$ e $s_{1}$ nas retas $r_{2}$ e $s_{2}$, respectivamente, com $\left(a_{1}, b_{1}\right)$ sendo levado em $\left(a_{2}, b_{2}\right)$. Podemos pensar, primeiramente, em aplicar a transformação $T_{1}$ e levar as retas $r_{1}$ e $s_{1}$ para os eixos $x$ e $y$, respectivamente e $\left(a_{1}, b_{1}\right)$ para a origem. Em seguida, aplicamos uma transformação que leva os eixos nas retas $r_{2}$ e $s_{2}$ e a origem em $\left(a_{2}, b_{2}\right)$, que nada mais é do que $T_{2}^{-1}$. Logo, $T=T_{2}^{-1} \circ T_{1}$. Ou seja, 


$$
\begin{aligned}
& T\left(\begin{array}{l}
x \\
y
\end{array}\right)=R_{-\theta_{2}} \circ\left(R_{\theta_{1}}\left(\begin{array}{l}
x \\
y
\end{array}\right)-\left(\begin{array}{l}
a_{1} \\
b_{1}
\end{array}\right)\right)-\left(\begin{array}{l}
a_{2} \\
b_{2}
\end{array}\right) \\
& T\left(\begin{array}{l}
x \\
y
\end{array}\right)=R_{-\theta_{2}} R_{\theta_{1}}\left(\begin{array}{l}
x \\
y
\end{array}\right)-R_{-\theta_{2}}\left(\begin{array}{l}
a_{1} \\
b_{1}
\end{array}\right)-\left(\begin{array}{l}
a_{2} \\
b_{2}
\end{array}\right)
\end{aligned}
$$

em que

$$
R_{-\theta_{2}} R_{\theta_{1}}=R_{\theta_{1}-\theta_{2}}=\left(\begin{array}{rr}
\cos \left(\theta_{1}-\theta_{2}\right) & -\sin \left(\theta_{1}-\theta_{2}\right) \\
\sin \left(\theta_{1}-\theta_{2}\right) & \cos \left(\theta_{1}-\theta_{2}\right)
\end{array}\right)
$$

Portanto, existe uma transformação (composição de rotação e translação) que leva um par de retas perpendiculares em outra e assim, conclui-se que as definições da Geometria Analítica satisfazem o postulado 4 de Euclides.

Proposição 6 A definição 1 (reta) satisfaz o quinto postulado de Euclides.

Prova. A primeira observação a ser feita para demonstrar a proposição acima é que duas retas são paralelas se não têm ponto em comum. Assim, considerando a definição 1 , isto acontece quando as equações do primeiro grau que representam as retas formam um sistema impossível. Ou seja, dadas duas retas paralelas $r$ e $s$ de equações $a x+b y=c$ e $a^{\prime} x+b^{\prime} y=c^{\prime}$, respectivamente, devemos ter $a^{\prime}=\lambda a, b^{\prime}=\lambda b$ e $c^{\prime} \neq \lambda c, \lambda \in \mathbb{R}$.

Por conseguinte, dada uma reta $r$ de equação $a x+b y=c$ e um ponto $P=\left\{\left(x_{P}, y_{P}\right)\right\}$, não incidente em $r$, a equação de uma reta $s$, paralela a $r$ e que passa por $P$, é dada por $a x+b y=d$, em que $d=a x_{P}+b y_{P}$. Afirmamos que esta é a única paralela a $r$ que passa por $P$.

De fato, suponha que exista outra reta $t$ paralela a $r$ e passando por $P$. A reta $t$ terá equação da forma $a x+b y=k, \operatorname{com} k \neq d$. Mas $P$ incide sobre $t$, donde segue que $a x_{P}+b y_{P}=k \neq d=a x_{P}+b y_{P}$ (contradição). Logo, existe apenas uma reta passando por $P$ e paralela a $r$, de modo que o postulado 5 se verifica com a definição de reta acima referida.

A principal motivação para o estudo na Geometria Analítica, no contexto desta dissertação, diz respeito à apresentação do teorema a seguir, devido a Descartes, que relaciona a construtibilidade de determinados pontos com operações,,$+- \times \mathrm{e} \div$ e raízes quadradas.

Teorema 1 (Descartes) Sejam dados os pontos $P_{1}=\left\{\left(a_{1}, b_{1}\right)\right\}, P_{2}=$ $\left\{\left(a_{2}, b_{2}\right)\right\}, \cdots, P_{n}=\left\{\left(a_{n}, b_{n}\right)\right\}$. Então, o ponto $Q=\{(\alpha, \beta)\}$ pode ser construído com régua e compasso a partir de $P_{1}, P_{2}, \cdots, P_{n}$ se, e somente se, $\alpha$ e $\beta$ forem o resultado de operações,,$+- \times e \div$ aplicadas às coordenadas 
$a_{1}, a_{2}, \cdots, a_{n}, b_{1}, b_{2}, \cdots, b_{n}$ e, ainda, da solução de um número finito de equações do primeiro e do segundo graus, envolvendo raízes quadradas de números reais positivos.

Prova. Uma construção com régua e compasso consiste em traçar retas a partir de determinados pontos, em desenhar círculos com centros e raios determinados, bem como em obter interseções entre duas retas, dois círculos ou entre retas e círculos.

Na prova da proposição 2, vimos que a equação da reta que passa pelos pontos $P_{1}=\left\{\left(a_{1}, b_{1}\right)\right\}$ e $P_{2}=\left\{\left(a_{2}, b_{2}\right)\right\}$ é dada por $\left(b_{2}-b_{1}\right) x-\left(a_{2}-a_{1}\right) y=a_{1} b_{2}-a_{2} b_{1}$; de modo análogo, são obtidas as equações das retas que passam por dois dos outros pontos dados.

Ainda, como visto na definição 3 , a equação de um círculo de centro $\{(a, b)\}$ e raio $r$ é dada por $(x-a)^{2}+(y-b)^{2}=r^{2}$. Esta equação tem termos do segundo grau em $x$ e em $y$.

Assim, para encontrarmos o ponto de interseção entre duas retas, devemos resolver um sistema de duas equações do primeiro grau, utilizando as operações ,,$+- \times \mathrm{e} \div$.

Já a interseção entre uma reta e um círculo envolve a resolução de um sistema em que uma das equações é do primeiro grau e a outra tem termos do segundo grau. Portanto, neste caso, o problema recai em uma equação quadrática, a qual envolve raiz quadrada em sua solução.

Para obtermos a interseção entre dois círculos, devemos subtrair uma das equações da outra, o que resultará na eliminação dos termos de segundo grau $x^{2}$ e $y^{2}$. Após obtermos uma relação entre os termos $x$ e $y$, devemos retornar a uma das equações de círculo e efetuar uma substituição de variável, que resultará numa equação do segundo grau, cuja solução envolve raízes quadradas.

O exposto acima significa que, para conseguirmos as coordenadas do ponto $Q=\{(\alpha, \beta)\}$ com régua e compasso, devemos, a partir dos pontos dados $\left(P_{1}, P_{2}, . ., P_{n}\right)$, resolver um número finito de equações lineares e quadráticas, com coeficientes dependendo das coordenadas pontos iniciais e com quantidades construídas a partir dos pontos dados, o que envolve a utilização de operações,,$+- \times$ e $\div$ e a extração de raízes quadradas.

Reciprocamente, provaremos que, dados os segmentos de medidas $1, a$ e $b$, é possível obter os segmentos de medida $a+b, b-a, a \cdot b, \frac{b}{a} \mathrm{e} \sqrt{a}$.

Os segmentos de medida $a+b$ e $b-a$ são obtidos traçando-os sobre a mesma reta; para a adição, a extremidade de um deve coincidir com a extremidade do outro e, para a diferença, os segmentos devem estar sobrepostos, com uma das extremidades coincidente (figura 2.10). 


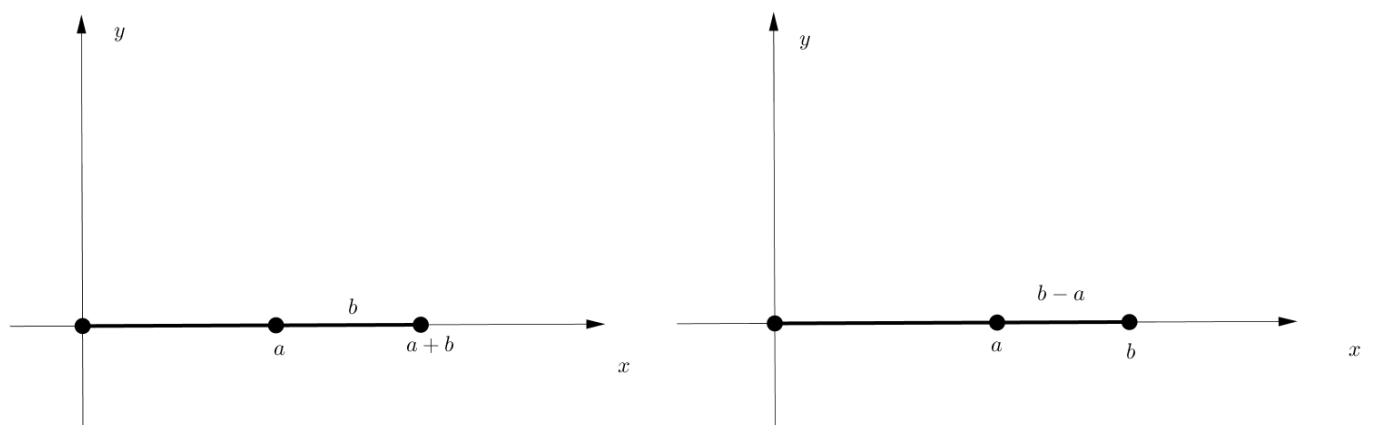

Figura 2.10: Adição e Diferença de Segmentos

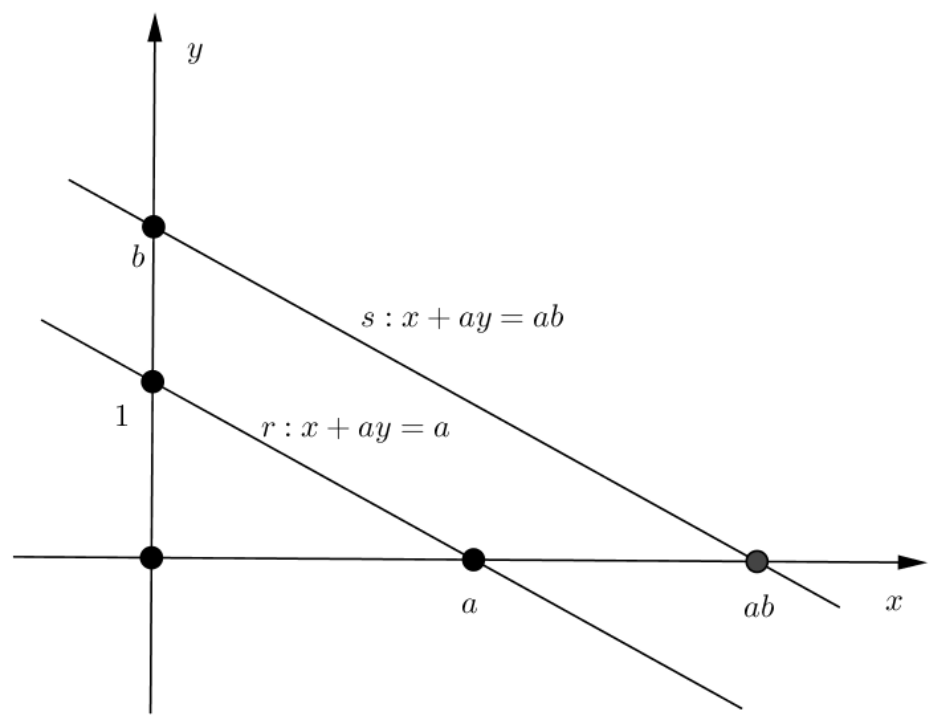

Figura 2.11: Obtenção do segmento de medida $a b$

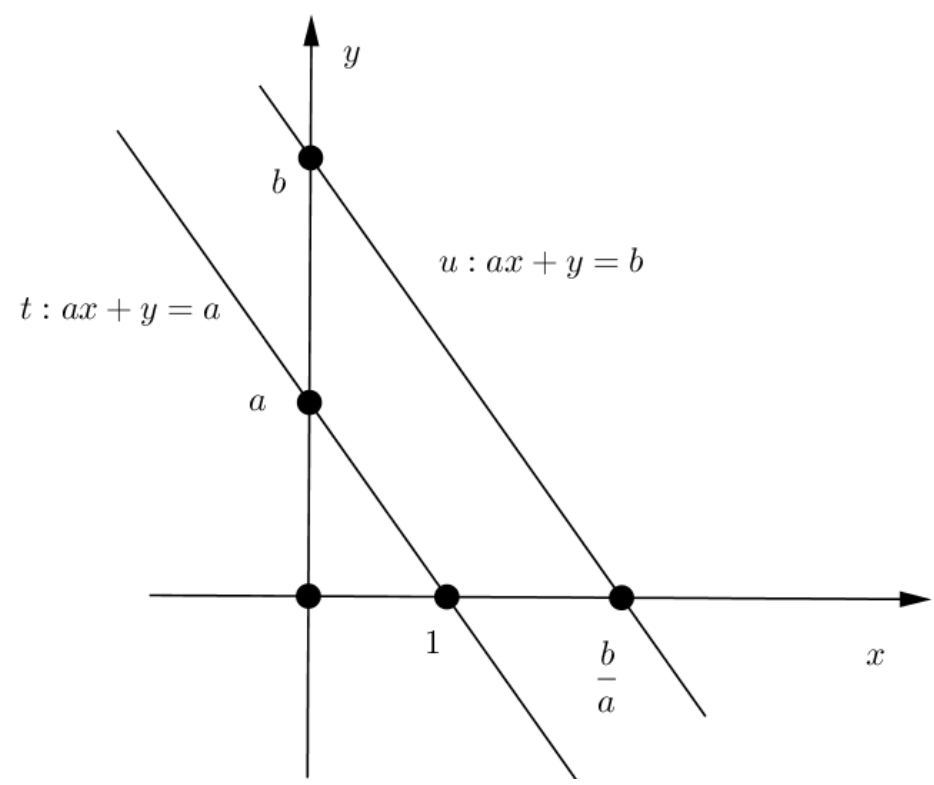

Figura 2.12: Obtenção do segmento de medida $\frac{b}{a}$ 
Para obtermos um segmento de medida a.b, devemos traçar, no plano com sistema de coordenadas, a reta $r$ que passa pelos pontos $\{(0,1)\}$ e $\{(a, 0)\}$, que tem equação $x+a y=a$. Depois, devemos obter a reta $s$ paralela a $r$ e que passa pelo ponto $\{(0, b)\}$ que terá equação da forma $x+a y=k$. É fácil de ver que $k=a b$. Assim, $s$ tem equação $x+a y=a b$. Também é claro que $s$ corta o eixo $x$ no ponto $\{(a b, 0)\}$ e, assim, obtemos o segmento de medida $a b$ (figura 2.11).

A estratégia para o segmento de medida $\frac{b}{a}$ é análoga: devemos traçar, no plano com sistema de coordenadas, a reta $t$ que passa pelos pontos $\{(1,0)\}$ e $\{(0, a)\}$, que tem equação $a x+y=a$ e a reta $u$, paralela a $t$, que passa pelo ponto $\{(0, b)\}$; a equação de $s$ é da forma $a x+y=l$, sendo fácil concluir que $l=b$. Logo, $u$ tem equação $a x+y=b$ e é claro que $u$ passa pelo ponto $\left\{\left(\frac{b}{a}, 0\right)\right\}$, conseguimos o segmento de medida $\frac{b}{a}$ procurado (figura 2.12).

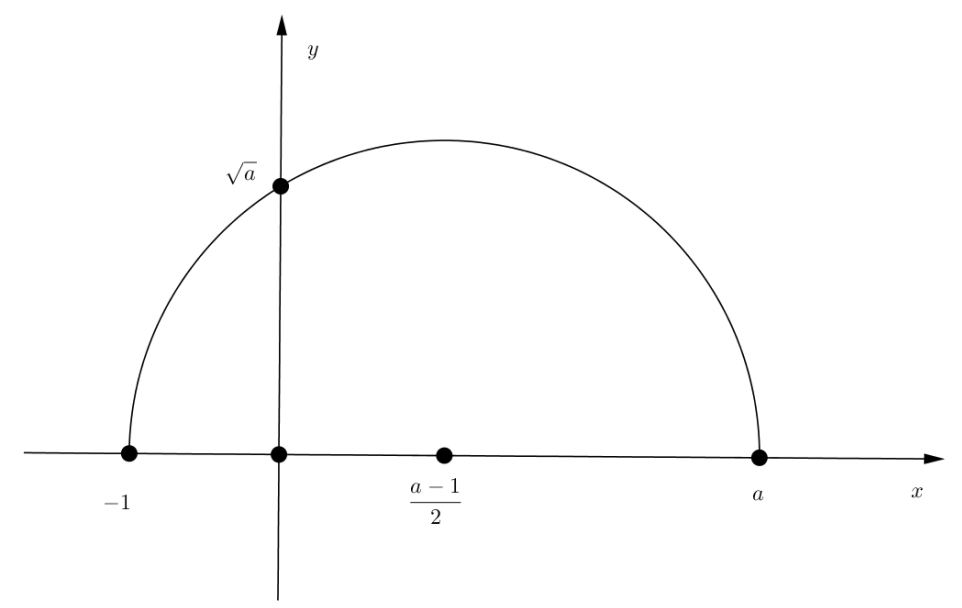

Figura 2.13: Obtenção do segmento de medida $\sqrt{a}$

Com relação ao segmento de medida $\sqrt{a}$, procedemos da seguinte forma (figura 2.13): traçamos um semicírculo com diâmetro de extremidades $\{(-1,0)\}$ e $\{(a, 0)\}$, que intersectará o eixo y acima da origem. O centro do círculo será o ponto médio do segmento referido, $\left\{\left(\frac{a-1}{2}, 0\right)\right\}$. O raio do círculo tem medida correspondente a metade do segmento entre os pontos $\{(-1,0)\}$ e $\{(a, 0)\}$, isto é, $\frac{a+1}{2}$. Portanto, o semicírculo tem equação

$$
\left[x-\left(\frac{a-1}{2}\right)\right]^{2}+y^{2}=\left(\frac{a+1}{2}\right)^{2}
$$

em que $y>0$. O ponto em que o semicírculo intersecta o eixo $x$, acima da origem, tem abscissa $x=0$. Determinaremos a ordenada de tal ponto: 


$$
\begin{gathered}
{\left[0-\left(\frac{a-1}{2}\right)\right]^{2}+y^{2}=\left(\frac{a+1}{2}\right)^{2}} \\
a^{2}-2 a+1+4 y^{2}=a^{2}+2 a+1 \\
4 y^{2}=4 a \Rightarrow y^{2}=a \Rightarrow y=\sqrt{a}
\end{gathered}
$$

Assim, encontramos um segmento de medida $\sqrt{a}$, o que finaliza a demonstração.

Uma observação importante é que seria possível obter, por construção, de forma bem simples, os segmentos de medida $a . b, \frac{b}{a}$ e $\sqrt{a}$, considerando resultados de Geometria Sintética: a semelhança de triângulos, o teorema de Tales e as relações métricas no triângulo retângulo.

É relevante destacar, ainda, que o teorema 1 fornece um critério algébrico para decidir sobre a possibilidade de uma dada construção geométrica. No entanto, tal resultado não estabelece um método para realizar a referida construção, caso seja possível.

\section{3}

\section{Resposta Moderna aos Problemas Clássicos de Construtibilidade}

Para que seja possível apresentar as respostas negativas aos clássicos problemas de construtibilidade, faz-se necessário, antes, introduzir alguns conceitos de natureza algébrica. Tentaremos nesta seção compendiar as principais definições e resultados, assumindo do leitor alguma familiaridade com os temas abordados. Referências para esta parte do texto podem ser encontradas em [8] e [9]. Neste último livro, é possível encontrar o tratamento algébrico dos problemas de construtibilidade com régua e compasso.

Definição 5 (Corpo) Seja $K$ um conjunto não-vazio em que são definidas duas operações binárias $+e \cdot$, denominadas adição e multiplicação, respectivamente. Dizemos que $(K,+, \cdot)$ é um corpo se são satisfeitas as seguintes propriedades:

(i) Associatividade da Adição. $\forall a, b, c \in K, a+(b+c)=(a+b)+c$;

(ii) Elemento Neutro da Adição. $\exists 0 \in K ; \forall a \in K, a+0=0+a=a$;

(iii) Elemento Simétrico. $\forall a \in K, \exists-a \in K ; a+(-a)=(-a)+a=0$;

(iv) Comutatividade da Adição. $\forall a, b \in K, a+b=b+a$;

(v) Associatividade da Multiplicação. $\forall a, b, c \in K, a \cdot(b \cdot c)=(a . b) . c$;

(vi) Elemento Neutro da Multiplicação. $\exists 1 \in K \backslash\{0\} ; \forall a \in K, a \cdot 1=$ $1 \cdot a=a$; 
(vii) Elemento Inverso. $\forall a \in K \backslash\{0\}, \exists a^{-1} \in K ; a \cdot a^{-1}=a^{-1} \cdot a=1$;

(viii) Comutatividade da Multiplicação. $\forall a, b \in K, a \cdot b=b \cdot a$;

(ix) Distributividade. $\forall a, b, c \in K, a \cdot(b+c)=a \cdot b+a \cdot c$.

São exemplos de corpos $\mathbb{Q}, \mathbb{R}$ e $\mathbb{C}$, com as operações de adição e multiplicação usuais. Como a propriedade (vii) não é satisfeita por $\mathbb{Z}$ (p.ex., $1 / 2 \notin \mathbb{Z})$, vemos que os números inteiros não formam um corpo. Conjuntos, como $\mathbb{Z}$, munidos de operações binárias que satisfazem os axiomas de corpo acima com a possível exceção de (vii) são chamados anéis. Um outro tipo de anel muito importante é o anel $K[x]$ dos polinômios na indeterminada $x$ com coeficientes no corpo $K$ :

$$
K[x]=\left\{c_{0}+c_{1} x+\cdots+c_{n} x^{n} \mid n \in \mathbb{N} \cup\{0\}, c_{0}, c_{1}, \cdots, c_{n} \in K\right\} .
$$

Outro exemplo importante de corpo no contexto do presente trabalho é o conjunto $E \subset \mathbb{R}$, dos comprimentos construtíveis com régua e compasso no plano euclideano a partir de um comprimento unitário pré-fixado, considerando como operações de adição e multiplicação as construções apresentadas quando da prova do teorema 1.

Definição 6 (Subcorpos e extensão de corpos) Seja $K$ um corpo. Um subconjunto $S \subseteq K$, é um subcorpo se, e somente se, $S$ é fechado com relação às operações $+e \cdot$ definidas para $K$ (i.e., $\forall a, b \in S, a+b \in S$ e $a \cdot b \in S$ ) e $(S,+, \cdot)$ é um corpo. Nesse, caso, dizemos também que o corpo $K$ é uma extensão do corpo $S$.

Para que um subconjunto $S$ de um corpo $K$ seja um subcorpo, é necessário e suficiente que contenha 0 e \pm 1 e que, para todos $a, b \in S, a+b \in S$, $a \cdot b \in S$, e, se $b \neq 0$, então $\frac{a}{b} \in S$. Aqui, $+\mathrm{e} \cdot$ são as operações definidas em $K$.

Como exemplo do exposto, observe que todas as inclusões em

$$
\mathbb{Q} \subset E \subset \mathbb{R} \subset \mathbb{C}
$$

são extensões de corpos (já a inclusão $\mathbb{Z} \subset \mathbb{Q}$, como vimos, não é uma extensão de corpos). Uma sequência de extensões concatenadas como essa é dita uma torre de corpos.

Para respondermos aos problemas clássicos de construtibilidade, necessitaremos de alguns teoremas básicos sobre extensões de corpos, em cujas demonstrações assumiremos do leitor alguma familiaridade com a teoria dos espaços vetoriais sobre um corpo abstrato $K$. As noções de que precisaremos 
(espaços vetoriais e seus subespaços, dependendência e independência linear, geradores, bases e dimensão) em geral são vistas no começo de qualquer graduação em Matemática, Ciências ou Engenharias em cursos de Álgebra Linear, onde normalmente se assume tacitamente que $K=\mathbb{R}$.

Definição 7 (Espaço Vetorial) Seja K um corpo. Um espaço vetorial sobre o corpo (de escalares) $K$ é um conjunto não-vazio $V$ munido de uma operação de adição $+: V \times V \rightarrow V$ e uma operação de multiplicação por escalar $\cdot: K \times V \rightarrow V$ com as seguintes propriedades:

(i) Associatividade da Adição. $\forall u, v, w \in V, u+(v+w)=(u+v)+w$;

(ii) Elemento Neutro da Adição. $\exists 0_{V} \in V ; \forall v \in V, v+0_{V}=0_{V}+v=v$;

(iii) Elemento Simétrico. $\forall v \in V, \exists-v \in V ; v+(-v)=(-v)+v=0_{V}$;

(iv) Comutatividade da Adição. $\forall u, v \in V, u+v=v+u$;

(v) Associatividade da Multiplicação por Escalar. $\forall \alpha, \beta \in K, u \in V$, $(\alpha \beta) \cdot v=(\alpha) \cdot(\beta . v)$;

(vi) Distributividade 1. $\forall \alpha, \beta \in K, u \in V,(\alpha+\beta) \cdot v=\alpha \cdot v+\beta \cdot v$;

(vii) Distributividade 2. $\forall \alpha \in K, u, v \in V, \alpha \cdot(u+v)=\alpha \cdot u+\alpha \cdot v$;

(viii) Elemento Neutro na Multiplicação por escalar. $\forall v \in V, 1_{K} \cdot v=v$.

Os conjuntos $\mathbb{R}, \mathbb{R}^{2}, \mathbb{R}^{3}, \ldots \mathbb{R}^{n}, \ldots$ são exemplos de espaços vetoriais sobre $\mathbb{R}$, onde

$$
\begin{aligned}
\mathbb{R}^{n} & =\underbrace{\mathbb{R} \times \cdots \times \mathbb{R}}_{n \text { fatores }} \\
& =\left\{\left(x_{1}, \cdots, x_{n}\right) \mid x_{1}, \cdots, x_{n} \in \mathbb{R}\right\}
\end{aligned}
$$

e a adição de vetores é feita coordenada a coordenada e a multiplicação por escalar é feita multiplicando todas as coordenadas pelo mesmo escalar. Note ainda que, identificando o número complexo $z=x+i y$ com o par ordenado $(x, y)$, temos que $\mathbb{C}=\mathbb{R}^{2}$ é um espaço vetorial sobre o corpo $\mathbb{R}$. De fato, é imediato verificar que, dada qualquer extensão de corpos $S \subseteq K$, temos que $K$ é um espaço vetorial sobre $S$ com relação às operações binárias de corpo + e $\cdot$ definidas em $K$.

É relevante expor, ainda, algumas definições relativas a espaços vetoriais.

Definição 8 (Dependência e Independência Linear) Seja $V$ um espaço vetorial sobre $K, r \in \mathbb{N}^{*}$ e sejam $v_{1}, v_{2}, \ldots, v_{r}$ vetores de $V$. Dizemos que os vetores $v_{1}, v_{2}, \ldots, v_{r}$ são linearmente dependentes sobre $K$ quando a equação

$$
\alpha_{1} \cdot v_{1}+\alpha_{2} \cdot v_{2}+\ldots+\alpha_{r} \cdot v_{r}=0_{V}
$$


admite em $K$ solução diferente da solução trivial $\alpha_{1}=\alpha_{2}=\ldots=\alpha_{r}=0_{K}$. Caso contrário, dizemos que os vetores $v_{1}, v_{2}, \ldots, v_{r}$ são linearmente independentes sobre $K$ (ou simplesmente LI).

Dado um subconjunto $X$ de $V$ (possivelmente infinito), dizemos que $X$ é linearmente dependente sobre $K$ quando existe um número finito $r$ de vetores $v_{1}, v_{2}, \ldots, v_{r} \in X$ linearmente dependentes sobre $K$. Caso contrário, dizemos que o subconjunto $X$ é linearmente indenpendente sobre $K$.

Definição 9 (Espaço Gerado) Seja $V$ um espaço vetorial sobre $K$ e seja $X$ um conjunto de vetores de $V$. Dizemos que $V$ é gerado por $X$ e escrevemos

$$
\operatorname{span}_{K}(X)=V
$$

quando todo $v \in V$ é uma combinação linear finita de elementos de $X$. Ou seja, existem $r \in \mathbb{N}^{*}, v_{1}, v_{2}, \ldots, v_{r} \in X$ e $\alpha_{1}, \alpha_{2}, \ldots, \alpha_{r} \in K$ tais que

$$
v=\alpha_{1} \cdot v_{1}+\alpha_{2} \cdot v_{2}+\ldots+\alpha_{r} \cdot v_{r} .
$$

Com base nas definições acima, estamos em condições de definir a base e a dimensão de um espaço vetorial.

Definição 10 (Base de um espaço Vetorial) Seja $V$ um espaço vetorial sobre $K$ e seja $X$ um conjunto de vetores de $V$ (possivelmente infinito). Dizemos que $X$ é uma base de $V$ sobre $K$ se $X$ é linearmente independente $e$ gera $V$.

Todo espaço vetorial possui uma base e quaisquer duas bases de um mesmo espaço vetorial sobre o mesmo corpo de escalares possuem o mesmo número de vetores. Omitiremos as provas desses dois resultados básicos da teoria dos espaços vetoriais.

Definição 11 (Dimensão de um Espaço Vetorial) Seja $V$ um espaço vetorial sobre o corpo $K$. A dimensão de $V$ sobre $K$, denotada por $\operatorname{dim}_{K} V$ é a cardinalidade (número de elementos) de qualquer de suas bases. No caso em que $V$ admite como base sobre $K$ um conjunto infinito $X$ de vetores, escreve$m o s \operatorname{dim}_{K} V=\infty$.

Por exemplo, não é difícil ver que $\operatorname{dim}_{\mathbb{R}} \mathbb{R}^{n}=n$. O leitor deve notar que a dimensão depende crucialmente do corpo de escalares $K$ que se considera. Por exemplo, como vimos acima, $\operatorname{dim}_{\mathbb{R}} \mathbb{C}=2$, mas é claro que $\operatorname{dim}_{\mathbb{C}} \mathbb{C}=1$.

As definições acima são relevantes pois, como vimos, se $K \subseteq L$ é uma extensão de corpo, então $L$ é um espaço vetorial sobre $K$. 
Definição 12 (Grau de uma Extensão de Corpo) Denotamos o grau de uma extensão de corpo $K \subseteq L$ por $[L: K]$ e a definimos como

$$
[L: K]=\operatorname{dim}_{K} L
$$

$A$ extensão $K \subseteq L$ é dita finita (resp., infinita) se seu grau $[L: K]$ é finito (resp., infinito).

Portanto, $\mathbb{C}$ é uma extensão de grau dois de $\mathbb{R}$. Outro exemplo de extensão quadrática é conjunto $\mathbb{Q}(\sqrt{2})=\{a+b \sqrt{2} \mid a, b \in \mathbb{Q}\}$. Cada elemento de $\mathbb{Q}(\sqrt{2})$ é escrito de forma única como combinação linear de 1 e $\sqrt{2}$ com coeficientes racionais, por causa da irracionalidade de $\sqrt{2}$. Portanto, $\{1, \sqrt{2}\}$ é uma base para $\mathbb{Q}(\sqrt{2})$ sobre $\mathbb{Q}$. Para mostrar que $\mathbb{Q}(\sqrt{2})$ assim definido é de fato um corpo, pode-se explorar a analogia com $\mathbb{C}=\mathbb{R}(i)=\{x+y i \mid x, y \in \mathbb{R}\}$. Interessante notar neste ponto que $\sqrt{2}$ é raiz da equação do segundo grau $x^{2}=2$, com coeficientes em $\mathbb{Q}$ e sem raizes em $\mathbb{Q}$; assim como a unidade imaginária $i$ é raiz da equação do segundo grau $x^{2}=-1$, com coeficientes em $\mathbb{R}$ e sem raizes em $\mathbb{R}$. Como veremos abaixo na proposição 7 , isso não é uma coincidência.

Definição 13 (Elemento Algébrico e Transcendente) Seja $K \subseteq L$ uma extensão de corpo. Dizemos que um elemento $\alpha \in L$ é algébrico sobre $K$ se existe um polinômio não-nulo

$$
p(x)=a_{n} x^{n}+\cdots+a_{1} x+a_{0} \in K[x] \backslash\{0\}
$$

com coeficientes $a_{0}, a_{1}, \cdots, a_{n} \in K$ tal que

$$
p(\alpha)=a_{n} \alpha^{n}+\cdots+a_{1} \alpha+a_{0}=0_{L} .
$$

Se $\alpha \in L$ não é algébrico sobre $K$, dizemos que $\alpha$ é transcendente sobre $K$.

Quando $K=\mathbb{Q}$ e L é uma extensão de $\mathbb{Q}$ contida em $\mathbb{C}$, dizemos simplesmente que $\alpha$ é um número algébrico, ou um número transcendente, conforme o caso.

Se todo elemento de $L$ é algébrico sobre $K$, dizemos que a extensão $K \subseteq L$ é algébrica. Caso contrário, dizemos que a extensão $K \subseteq L$ é transcendente.

Como vimos acima, $\sqrt{2}$ e $i$ são números algébricos. Números transcendentes famosos são $\pi$ e $e$. Sabe-se, graças ao Teorema de Lindemann-Weierstrass, que os números da forma $e^{a}, \ln (a), \sin (a), \cos (a)$ e $\tan (a)$ com $a$ algébrico nãonulo (e $a \neq 1$, no caso do logaritmo) são todos transcendentes. Esse mesmo teorema garante que a solução de $\cos (x)=x$ é um número transcendente. Sabe-se 
ainda, graças ao teorema de Gelfond-Schneider (ver teorema 21), que os números $e^{\pi}$ e $2^{\sqrt{2}}$ são transcendentes (esses números ficaram conhecidos como constantes de Gelfond-Schneider). Os teoremas de Lindemann-Weierstrass e Gelfond-Schneider encontram uma generalização comum no teorema de Baker. Esses resultados têm sido uma grande fonte de números transcendentes conhecidos, embora muitos outros tenham sido obtidos por outros métodos, como os números de Liouville $\sum_{k=1}^{\infty} \beta^{k !}$, com $\beta$ algébrico e $0<\beta<1$, obtidos estudando a eficácia das aproximações diofantinas de números algébricos irracionais (Teorema de Liouville). A constante de Liouville $\lambda=0,110001000 \cdots$ (faça $\beta=1 / 10$ na soma infinita acima) foi o primeiro número transcendente conhecido. Embora mais difíceis de serem exibidos, os números transcendentes formam, num certo sentido matematicamente preciso, a maioria esmagadora dos números reais, já que o conjunto dos números algébricos é enumerável. Neste trabalho, não apresentaremos prova de nenhum critério de transcendência, como os citados acima, embora façamos uso de alguns deles no capítulo 4 (teoremas 21 e 24). Remetemos o leitor interessado a [6].

Proposição 7 São verdadeiras as seguintes afirmações a respeito de uma extensão de corpo $K \subseteq L$ e de um elemento $\alpha \in L \backslash\{0\}$ algébrico sobre $K$.

(1) Existe um único polinômio mônico

$$
p_{\alpha}(x)=x^{n_{\alpha}}+a_{n_{\alpha}-1} x^{n_{\alpha}-1}+\cdots+a_{1} x+a_{0} \in K[x] \backslash\{0\}
$$

com coeficientes em $K$ satisfazendo $p_{\alpha}(\alpha)=0$ que tem grau $n_{\alpha} \geq 1$ mais baixo dentre todos os polinômios em $K[x] \backslash\{0\}$ que têm $\alpha$ como raiz em $L$.

(2) $p_{\alpha}(x)$ é irredutivel sobre $K[x]$.

(3) Seja $K(\alpha)$ a menor subextensão de $K \subseteq L$ contendo $\alpha$, ou seja:

$$
K(\alpha)=\bigcap_{\substack{K \subseteq M \subseteq L \\ \alpha \in M}} M
$$

Então,

$$
K(\alpha)=K[\alpha]=\{p(\alpha) \mid p(x) \in K[x]\} .
$$

(4) Sendo $n_{\alpha}=\operatorname{grau}_{x} p_{\alpha}(x)$, temos que $\left\{1, \alpha, \cdots, \alpha^{n_{\alpha}-1}\right\}$ é uma base de $K(\alpha)$ 
sobre K. Em particular,

$$
\begin{aligned}
K(\alpha) & =\operatorname{span}_{K}\left(1, \alpha, \cdots, \alpha^{n_{\alpha}-1}\right) \\
& =\left\{c_{0}+c_{1} \alpha+\cdots+c_{n_{\alpha}-1} \alpha^{n_{\alpha}-1} \mid c_{0}, c_{1}, \cdots, c_{n_{\alpha}-1} \in K\right\} .
\end{aligned}
$$

(5) $[K(\alpha): K]=n_{\alpha}$.

Prova. (1) Como o conjunto $\mathbb{N}$ é bem ordenado por $\leq$, seu subconjunto

$$
G_{\alpha}=\left\{n \in \mathbb{N} \mid \exists p(x) \in K[x] \backslash 0 \text { tal que } \operatorname{grau}_{x} p(x)=n \text { e } p(\alpha)=0\right\}
$$

dos graus de polinômios com coeficientes em $K$ que se anulam em $\alpha$ possui um menor elemento, que chamaremos $n_{\alpha}$. Sejam $p(x), q(x) \in K[x]$ polinômios mônicos de grau $n_{\alpha}$ que se anulam em $\alpha$. Se tivéssemos $p(x) \neq q(x)$, então $p(x)-q(x) \in K[x]$ seria um polinômio de grau menor do que $n_{\alpha}$ se anulando em $\alpha$, o que contrariaria a minimalidade de $n_{\alpha}$ em $G_{\alpha}$. Resta admitir que $p(x)=q(x)$.

(2) Suponha por absurdo que se tivesse $p_{\alpha}(x)=f(x) \cdot g(x)$, com $f(x), g(x) \in$ $K[x] \backslash K$. Então, como $L$ é corpo, teríamos $f(\alpha)=0$ ou $g(\alpha)=0$. Mas como $1 \leq \operatorname{grau}_{x} f(x)<n_{\alpha}$ e $1 \leq \operatorname{grau}_{x} g(x)<n_{\alpha}$, isso contrariaria a minimalidade de $n_{\alpha}$ em $G_{\alpha}$. Portanto, $p_{\alpha}(x)$ é irredutível em $K[x]$.

(3) É evidente que $K[\alpha] \subseteq K(\alpha)$ e que $K[\alpha]$ é fechado com relação às operações + e · de $L$, além de conter obviamente $0, \pm 1 \in L$. Basta então mostrar que, dado $h(x) \in K[x] \operatorname{com} h(\alpha) \neq 0$, temos $h(\alpha)^{-1} \in K[\alpha]$ para verificar que $K[\alpha]$ é um subcorpo de $L$. Seguirá da definição e da inclusão óbvia $K[\alpha] \subseteq K(\alpha)$ que $K(\alpha)=K[\alpha]$. Vamos à prova: dado $h(x) \in K[x] \operatorname{com} h(\alpha) \neq 0$, temos que $h(x)$ não é divisível por $p_{\alpha}(x)$ em $K[x]$. Como $p_{\alpha}(x)$ é irredutível em $K[x]$, temos que $\operatorname{mdc}_{K[x]}\left(p_{\alpha}(x), h(x)\right)=1$, donde segue que existem $f(x), g(x) \in K[x]$ tais que

$$
f(x) \cdot p_{\alpha}(x)+g(x) \cdot h(x)=1 .
$$

Avaliando essa expressão polinomial em $\alpha$, temos $g(\alpha) \cdot h(\alpha)=1$, ou seja: $g(\alpha)=h(\alpha)^{-1} \in K[\alpha]$, como queríamos demonstrar.

(4) É evidente que $1, \alpha, \cdots, \alpha^{n_{\alpha}-1}$ são linearmente independentes sobre $K$, pois uma relação de dependência linear

$$
c_{0}+c_{1} \alpha+\cdots+c_{n_{\alpha}-1} \alpha^{n_{\alpha}-1}=0
$$

entre esses elementos de $L$ com coeficientes em $K$ (não todos nulos), forneceria um polinômio $p(x)=c_{0}+c_{1} x+\cdots+c_{n_{\alpha}-1} x^{n_{\alpha}-1} \in K[x] \backslash\{0\}$ de grau menor que 
$n_{\alpha}$ se anulando em $\alpha$, o que estaria em contradição com a minimalidade de $n_{\alpha}$ em $G_{\alpha}$. Resta então provar que os elementos $1, \alpha, \cdots, \alpha^{n_{\alpha}-1} \in L$ geram $K(\alpha)=$ $K[\alpha]$ sobre $K$. De fato, basta mostrar que $\alpha^{n_{\alpha}} \in \operatorname{span}_{K}\left(1, \alpha, \cdots, \alpha^{n_{\alpha}-1}\right)$. Para isso, basta notar que, por definição, temos

$$
p_{\alpha}(\alpha)=\alpha^{n_{\alpha}}+a_{n_{\alpha}-1} \alpha^{n_{\alpha}-1}+\cdots+a_{1} \alpha+a_{0}=0,
$$

donde segue que

$$
\alpha^{n_{\alpha}}=-a_{n_{\alpha}-1} \alpha^{n_{\alpha}-1}-\cdots-a_{1} \alpha-a_{0}
$$

é combinação linear de $1, \alpha, \cdots, \alpha^{n_{\alpha}-1}$ com coeficientes em $K$. Portanto, $\left\{1, \alpha, \cdots, \alpha^{n_{\alpha}-1}\right\}$ é uma base de $K(\alpha)$ sobre $K$ formada por $n_{\alpha}$ elementos. Isso prova (4) e (5).

A proposição 7 explica porque escrevemos alguns parágrafos acima que $\mathbb{Q}(\sqrt{2})=\{a+b \sqrt{2} \mid a, b \in \mathbb{Q}\}$, obtendo $[\mathbb{Q}(\sqrt{2}): \mathbb{Q}]=2$.

Ainda no espírito da proposição 7, cumpre observar que toda extensão finita é algébrica e, portanto, toda extensão transcendente é infinita (assim, temos p.ex. que $[\mathbb{Q}(\pi): \mathbb{Q}]=\infty)$. Com efeito, suponha a extensão $K \subseteq L$ finita de grau $n$ e considere um elemento $\alpha \in L$. A dependência linear

$$
c_{0} \cdot 1+c_{1} \alpha+\cdots+c_{n-1} \alpha^{n-1}+c_{n} \alpha^{n}=0
$$

(com coeficentes $c_{0}, \cdots, c_{n} \in K$ não todos nulos) entre os $n+1$ elementos $1, \alpha, \alpha^{2}, \cdots, \alpha^{n} \in L$ fornece um polinômio

$$
p(x)=c_{0}+c_{1} x+\cdots+c_{n-1} x^{n-1}+c_{n} x^{n} \in K[x] \backslash\{0\}
$$

que se anula em $\alpha$. Portanto, $\alpha \in L$ é algébrico sobre $K$.

Não são verdadeiras, entretanto, as implicações recíprocas: existem extensões algébricas infinitas. De fato, vamos provar que o conjunto dos números algébricos $\mathbb{A}$ é um corpo, e, portanto, uma extensão algébrica infinita de $\mathbb{Q}$. Para isso, lançaremos mão do seguinte importante resultado, que será utilizado ainda para dar uma caracterização algébrica dos pontos construtíveis no plano euclideano (teorema 2). Essa caracterização permitirá concluir que também a extensão $\mathbb{Q} \subset E$ (onde $E$ é o corpo dos comprimentos de segmentos construtíveis a partir do segmento unitário) é algébrica e infinita.

Proposição 8 Considere a torre de corpos $K \subseteq L \subseteq M$. A extensão $K \subseteq M$ é finita se, e somente se, as extensões $K \subseteq L$ e $L \subseteq M$ são ambas finitas. 
Nesse caso, tem-se a igualdade

$$
[M: K]=[M: L] \cdot[L: K]
$$

Prova. É evidente que, se $[M: K]<\infty$, então $[M: L]<\infty$ e $[L: K]<\infty$, pois $L$ é um subespaço vetorial de $M$ sobre o corpo de escalares $K$ e, obviamente, $\operatorname{dim}_{L} M \leq \operatorname{dim}_{K} M$. Podemos então supor sem perda de generalidade que as extensões $K \subseteq L$ e $L \subseteq M$ são ambas finitas. Sejam $[M: L]=m$ e $[L: K]=n$ e tome

$$
\mathfrak{B}_{L}^{M}=\left\{\alpha_{1}, \cdots, \alpha_{m}\right\}
$$

e

$$
\mathfrak{B}_{K}^{L}=\left\{\beta_{1}, \cdots, \beta_{n}\right\},
$$

onde $\mathfrak{B}_{L}^{M}$ e $\mathfrak{B}_{K}^{L}$ são, respectivamente, bases de $M$ sobre $L$ e de $L$ sobre $K$. Afirmamos que o conjunto

$$
\mathfrak{B}_{K}^{M}=\mathfrak{B}_{L}^{M} \cdot \mathfrak{B}_{K}^{L}=\left\{\alpha_{1} \cdot \beta_{1}, \cdots, \alpha_{1} \cdot \beta_{n}, \cdots, \alpha_{m} \cdot \beta_{1} \cdots, \alpha_{m} \cdot \beta_{n}\right\},
$$

formado por todos os produtos de elementos de $\mathfrak{B}_{L}^{M}$ e $\mathfrak{B}_{K}^{L}$, é uma base de $M$ sobre $K$. De fato, temos

$$
M=\operatorname{span}_{K} \mathfrak{B}_{K}^{M}
$$

pois, dado $\alpha \in M$, existem $a_{1}, \cdots, a_{m} \in L$ tais que

$$
\alpha=\sum_{i=1}^{m} a_{i} \cdot \alpha_{i}
$$

Agora, para cada $a_{i} \in L$, existem $b_{i 1}, \cdots, b_{i n} \in K$ tais que

$$
a_{i}=\sum_{j=1}^{n} b_{i j} \beta_{j}
$$

Portanto, temos

$$
\alpha=\sum_{i=1}^{m} \sum_{j=1}^{n} b_{i j} \cdot\left(\alpha_{i} \cdot \beta_{j}\right) \in \operatorname{span}_{K} \mathfrak{B}_{K}^{M}
$$

Falta ver que $\mathfrak{B}_{K}^{M}$ é linearmente independente. Dada uma combinação linear nula

$$
\sum_{i=1}^{m} \sum_{j=1}^{n} c_{i j} \cdot\left(\alpha_{i} \cdot \beta_{j}\right)=0
$$

dos elementos $\alpha_{i} \cdot \beta_{j}$ de $M$ com coeficientes $c_{i j}$ em $K$, podemos reescrevê-la usando a associatividade, a distributividade e a comutatividade em $M$ como 
uma combinação linear nula

$$
\sum_{i=1}^{m}\left(\sum_{j=1}^{n} c_{i j} \cdot \beta_{j}\right) \cdot \alpha_{i}=0
$$

$\operatorname{dos} \alpha_{i} \in M$ com coeficientes

$$
\sum_{j=1}^{n} c_{i j} \cdot \beta_{j} \in L
$$

Pela independência linear dos elementos $\alpha_{1}, \cdots, \alpha_{m}$ de $M$ sobre $L$, temos que esses coeficientes devem ser todos nulos:

$$
\forall i \in\{1, \cdots, m\}, \quad \sum_{j=1}^{n} c_{i j} \cdot \beta_{j}=0 .
$$

Agora, pela independência linear dos elementos $\beta_{1}, \cdots, \beta_{n}$ de $L$ sobre $K$, temos também que os coeficientes $c_{i j} \in K$ devem ser todos nulos, o que completa a prova.

Como aplicação da proposição 8 acima, podemos provar que o conjunto $\mathbb{A}$ dos números algébricos é um subcorpo de $\mathbb{C}$, donde uma extensão algébrica infinita de $\mathbb{Q}$. De fato, sejam $\alpha, \beta \in \mathbb{A}$ e vamos mostrar que $\alpha+\beta, \alpha \cdot \beta \in \mathbb{A} \mathrm{e}$ que, se $\beta \neq 0$, então $\beta^{-1} \in \mathbb{A}$. Considere

$$
\mathbb{Q}(\alpha, \beta)=\bigcap_{\substack{\mathbb{Q} \subseteq K \subseteq \mathbb{C} \\ \alpha, \beta \in K}} K,
$$

o menor subcorpo de $\mathbb{C}$ que contém $\mathbb{Q}$ e os números $\alpha$ e $\beta$. Não é difícil verificar que

$$
\mathbb{Q}(\alpha, \beta)=(\mathbb{Q}(\alpha))(\beta)=(\mathbb{Q}(\beta))(\alpha),
$$

onde os corpos $\mathbb{Q}(\alpha)$ e $\mathbb{Q}(\beta)$ são definidos de maneira análoga e foram introduzidos abstratamente na afirmação (3) da proposição 7 acima. Assim, podemos considerar a torre de corpos

$$
\mathbb{Q} \subseteq \mathbb{Q}(\alpha) \subseteq \mathbb{Q}(\alpha, \beta)
$$

Pela proposição 7 (afirmações 4 e 5 ) a extensão $\mathbb{Q} \subseteq \mathbb{Q}(\beta)$ é finita (assim como $\mathbb{Q} \subseteq \mathbb{Q}(\alpha)$, a propósito). Com maior razão, portanto, será finita a extensão $\mathbb{Q}(\alpha) \subseteq \mathbb{Q}(\alpha, \beta)$. Assim, pela proposição 8, temos que a extensão $\mathbb{Q} \subseteq \mathbb{Q}(\alpha, \beta)$ é finita, e portanto, algébrica. Como obviamente $\alpha+\beta, \alpha \cdot \beta \in \mathbb{Q}(\alpha, \beta)$ e, se $\beta \neq 0$, então $\beta^{-1} \in \mathbb{Q}(\alpha, \beta)$, segue que esses números são algébricos, como queríamos demonstrar. 
Para ver que a extensão $\mathbb{Q} \subset \mathbb{A}$ é infinita, basta observar que existem números algébricos $\alpha \in \mathbb{A}$ de grau $n_{\alpha}$ arbitrariamente alto sobre $\mathbb{Q}$, donde segue que existem subextensões $\mathbb{Q} \subsetneq \mathbb{Q}(\alpha) \subsetneq \mathbb{A}$ tais que $[\mathbb{Q}(\alpha): \mathbb{Q}]=n_{\alpha}$ é tão grande quanto se queira. Por exemplo, podemos considerar a sequência de polinômios $p_{n}(x)=x^{n}-p \in \mathbb{Z}[x]$ irredutíveis em $\mathbb{Q}[x]$, com $p$ primo, cujos zeros reais são as raízes $n$-ésimas de $p$. A irredutibilidade dos polinômios $p_{n}(x)$ segue, por exemplo, do critério de Eisenstein. Podemos pensar também na sequência de polinômios ciclotômicos $\Phi_{p}(x) \in \mathbb{Z}[x]$ de graus $p$ primos, cujos zeros são as raizes $p$-ésimas complexas da unidade. Pode-se mostrar que esses polinômios são irredutíveis em $\mathbb{Q}[x]$ pelo mesmo critério de Eisenstein (após uma mudança de variável conveniente). Remetemos o leitor interessado a [8].

Agora, podemos retomar a questão relativa à construtibilidade com régua e compasso. Pelo Teorema 1, concluímos que um ponto $Q=\{(\alpha, \beta)\}$, no plano cartesiano, pode construído com régua e compasso se e somente se este pode ser obtido, a partir das coordenadas dos pontos inicias $P_{1}, P_{2}, \ldots, P_{n}$, por meio de operações,,$+- \times$ e $\div$ (operações de corpos) e de extrações de raízes quadradas de números positivos.

Caso os pontos iniciais tenham como coordenadas apenas números racionais, então $\alpha$ e $\beta$ serão elementos do corpo de construtíveis $E$, que são obtidos a partir de operações de corpos e extrações de raízes quadradas dos elementos de $\mathbb{Q}$. Portanto, o ponto $Q$ pode se construído com régua e compasso no plano cartesiano se, e somente se, $\alpha$ e $\beta$ são números construtíveis.

O teorema a seguir, e o respectivo corolário, estabelecem critério de construtibilidade de números que será fundamental para provar a impossibilidade de solução dos problemas clássicos no contexto da Geometria Euclideana.

Teorema 2 Um número $\alpha \in \mathbb{R}$ é construtivel no plano euclideano se, e somente se, existe uma torre de subcorpos de $\mathbb{R}$,

$$
\mathbb{Q}=F_{0} \subseteq F_{1} \subseteq \ldots \subseteq F_{k} \subseteq \mathbb{R}
$$

tal que $\alpha \in F_{k}$ e $F_{i}=F_{i-1}\left(\sqrt{a_{i}}\right)$ para algum $a_{i} \in F_{i-1}$.

Prova. Suponhamos, primeiramente, $\alpha$ construtível. Escrevamos $\alpha$ como resultado de um número finito de operações de corpos e raízes quadradas. Assim, cada vez que aparecer uma raiz quadrada em tal escrita, defina recursivamente $F_{i}$ como o corpo decorrente da adjunção de tal raiz quadrada ao corpo anterior. Assim, obtemos uma torre de corpos na forma enunciada. 
Reciprocamente, se $\alpha \in F_{k}$, é claro que, pela forma como construída a torre de corpos, $\alpha$ é o resultado de operações de corpos e raízes quadradas. Logo, $\alpha$ é construtível no plano euclideano.

Corolário 1 Seja $\alpha \in \mathbb{R}$ construtivel. Então, $[\mathbb{Q}(\alpha): \mathbb{Q}]=2^{n}$ para algum $n \in \mathbb{N}$.

Prova. Ao acrescentarmos, a uma extensão de corpo, a raiz quadrada de um número que não é um quadrado perfeito, obtemos uma extensão de corpo de grau 2. Se todas as extensões de corpo do teorema 2 forem não-triviais, então devemos ter $\left[F_{k}: \mathbb{Q}\right]=2^{k}$. Uma vez que $\mathbb{Q} \subseteq \mathbb{Q}(\alpha) \subseteq F_{k}$ e se tem a multiplicatividade dos graus de extensões de corpos encadeadas (proposição 8), concluímos que $[\mathbb{Q}(\alpha): \mathbb{Q}]$ é da forma $2^{n}, n \in \mathbb{N}$.

Como todos os números construtíveis no plano euclideano são resultados de operações de corpos e de extrações de raízes quadradas, é bem claro que todos os números que podem ser construídos com régua e compasso são algébricos.

De posse dos resultados acima, estamos em condições de dar resposta aos problemas clássicos de construtibilidade.

Teorema 3 Não é possível duplicar um cubo, com régua e compasso, na Geometria Euclideana.

Prova. Considere que o cubo dado tem aresta $a$. Deste modo, para que se tenha um novo cubo que volume corresponde ao dobro do sólido original, devemos ter $b^{3}=2 . a^{3}$, o que implica $b=\sqrt[3]{2} a$. Portanto, $b$ pode ser construído com régua e compasso se, e somente se, $\sqrt[3]{2}$ é construtível.

Queremos provar que $\sqrt[3]{2}$ não é construtível. De fato, $\sqrt[3]{2}$ é raiz do polinômio $p(x)=x^{3}-2$. Provaremos que $p(x)$ é irredutível em $\mathbb{Q}$.

Suponha, por absurdo, que $p(x)$ não seja irredutível no conjunto dos racionais. Assim, o polinômio teria uma raiz em $\mathbb{Q}$. Seja $\frac{c}{d}$, fração irredutível, $c, d \in$ $\mathbb{Z} \backslash\{0\}$, a referida raiz. Daí, seguiria que

$$
\frac{c^{3}}{d^{3}}-2=0 \Rightarrow c^{3}=2 d^{3}
$$

Da igualdade acima, concluímos que $c^{3}$ é par, o que acarreta $c$ par e, consequentemente, $2^{3} \mid c^{3}$. Isto implica que $c^{3}=2^{3} k, k \in \mathbb{Z}$ (I). Substituindo (I) na igualdade acima, chegamos a

$$
2^{3} k=2 d^{3} \Rightarrow 4 k=d^{3}
$$


A última equação acima implica que $d^{3}$ é par, donde-se conclui que $d$ também é par. Tal fato contradiz a hipótese de que $\frac{c}{d}$ é fração irredutível. Logo $p(x)$ é irredutível em $\mathbb{Q}$.

Como $p(x)$ é irredutível em $\mathbb{Q}$ e tem grau 3 , concluímos, pela proposição 7, que $[\mathbb{Q}(\sqrt[3]{2}): \mathbb{Q}]=3$. Por via de consequência $\sqrt[3]{2}$ não é construtível, tendo em vista o corolário 1 do teorema 2 .

Por fim, concluímos que, como $\sqrt[3]{2}$ não é construtível, $b$ não pode ser construído com régua e compasso, o que prova o resultado.

Teorema 4 Não é possível, de modo geral, realizar a trissecção de um ângulo, com régua e compasso, na Geometria Euclideana.

Prova. Para a demonstração do resultado acima, é necessário esclarecer, primeiramente, que um ângulo $\alpha$ é construtível se, e somente se, suas funções trigonométricas podem ser construídas com régua e compasso. Para verificar este fato, basta pensar num triângulo retângulo com hipotenusa 1 e um dos ângulos de medida $\alpha$, que terá catetos de medida $\sin \alpha$ e $\cos \alpha$. Os demais casos acabam por reduzir-se ao exposto.

O problema da trissecção do ângulo é um pouco diverso do problema da duplicação do cubo, pois, em alguns casos, é possível construir um ângulo que tenha medida equivalente a um terço da de um ângulo dado. Por exemplo, se o ângulo dado for reto, para obter, por construção, um ângulo que tem medida $\frac{\pi}{6}$, pode-se construir um triângulo equilátero e, posteriormente, traçar a bissetriz de um dos seus ângulos internos.

Mostraremos que não é possível, de modo geral, trissectar um ângulo, provando que não é possível realizar a trissecção para o caso particular em que o ângulo tem medida $\frac{\pi}{3}$. É sabido que $\cos 3 \theta=4 \cos ^{3} \theta-3 \cos \theta$. Se $\theta=\frac{\pi}{9}$, tem-se que

$$
\begin{gathered}
\cos \frac{\pi}{3}=4 \cos ^{3} \frac{\pi}{9}-3 \cos \frac{\pi}{9} \\
4 \cos ^{3} \frac{\pi}{9}-3 \cos \frac{\pi}{9}=\frac{1}{2} \\
8 \cos ^{3} \frac{\pi}{9}-6 \cos \frac{\pi}{9}-1=0
\end{gathered}
$$

Fazendo $x=2 \cos \frac{\pi}{9}$, chegamos à equação polinomial.

$$
x^{3}-3 x-1=0
$$

Provaremos que o polinômio $q(x)=x^{3}-3 x-1$ é irredutível em $\mathbb{Q}$. 
Suponhamos, por absurdo, que $q(x)$ seja redutível em $\mathbb{Q}$. Deste modo, $q(x)$ teria uma raiz no conjunto dos racionais, digamos $\frac{a}{b}$, fração irredutível com $a, b \in \mathbb{Z} \backslash\{0\}$. Substituindo $x$ por $\frac{a}{b}$ e realizando as operações necessárias, chegamos à equação

$$
a^{3}-3 a b^{2}-b^{3}=0
$$

Da igualdade acima, concluímos que todo fator primo de $a$ deveria ser também fator primo de $b$. Como, por hipótese, $a$ e $b$ são primos entre si, devemos ter $a= \pm 1$ e $b= \pm 1$, donde segue que $\frac{a}{b}= \pm 1$. No entanto, 1 e -1 não são raízes do polinômio $q(x)$, como é possível verificar por inspeção. Portanto, $q(x)$ é irredutível em $\mathbb{Q}$.

Assim, considerando a proposição 7 e o corolário 1 do teorema 2, concluímos que $\left[\mathbb{Q}\left(\cos \frac{\pi}{9}\right): \mathbb{Q}\right]=3$ e que, por consequência, $\cos \frac{\pi}{9}$ não é construtível. Logo, o ângulo de medida $\frac{\pi}{9}$ não pode ser construído com régua e compasso.

Teorema 5 O problema da quadratura do círculo não tem solução com régua e compasso na Geometria Euclideana.

Prova. Considere um círculo de raio $r$, que tem área $\pi r^{2}$. Queremos construir um quadrado de lado $a$, que tenha a mesma área do círculo, o que resulta em $a^{2}=\pi r^{2}$. Deste modo, $a=r \sqrt{\pi}$, donde se conclui que $a$ é construtível se, e somente se, $\sqrt{\pi}$ é construtível. No entanto, $\pi$ é transcendente e, por via de consequência, não pode ser construído com régua e compasso, já que todo número construtível é algébrico.

Como $\pi$ não é construtível, segue que $\sqrt{\pi}$ também não é construtível, o que resulta na impossibilidade de construção, com régua e compasso, na Geometria Euclideana, de um quadrado com a mesma área que um círculo dado.

A prova de que $\pi$ é um número transcendente está muito além dos objetivos deste trabalho, pois depende de conceitos de Análise, e não será aqui apresentada. Uma demonstração deste fato pode ser encontrada em [10].

Após a apresentação das respostas negativas aos três problemas problemas clássicos na Geometria Euclideana, é possível conjecturar sobre a existência de solução para tais problemas em outras geometrias, que apresentam noções de distância e áreas completamente diferentes. É o que faremos no capítulo 4, no âmbito da Geometria Hiperbólica.

Para encerrar esta seção, apresentaremos um famoso resultado devido a Gauss e Wentzel, que diz respeito à construtibilidade de polígonos regulares no plano euclideano $\left(\mathbb{E}^{2}\right)$. 
Teorema 6 (Gauss-Wentzel) Um polígono regular de $n$ lados pode ser construído com régua e compasso em $\mathbb{E}^{2}$ se, e somente se, $n$ é da forma

$$
2^{k} \cdot F_{i_{1}} \cdot F_{i_{2}} \ldots F_{i_{r}}
$$

em que os $F_{i_{j}}$ são números primos de Fermat $\left(F_{l}=2^{2^{l}}+1\right)$.

Uma referência para este resultado pode ser encontrada em [9], p. 258.

O interesse de tal teorema, neste trabalho, diz respeito ao fato de que, se $n$ é um número da forma enunciada no teorema acima, não apenas o polígono regular de $n$ lados pode ser construído com régua e compasso, mas também o ângulo central de medida $\frac{2 \pi}{n}$. Este fato terá extrema relevância quando for apresentada a demonstração do teorema que estabelece as condições necessárias e suficientes para que um quadrado e um círculo de mesma área possam ser construídos com régua e compasso no plano hiperbólico, como veremos no Capítulo 4.

A seguir, serão apresentadas algumas definições e demonstrações da Geometria Tradicional que serão necessárias ao estudo da Geometria Hiperbólica, sobretudo do modelo de Poincaré, as quais dizem respeito à inversão circular.

\section{4}

\section{Inversão Circular}

Definição 14 Dado um círculo de centro $O$ e um ponto $P$ qualquer, dizemos que $P^{\prime}$ é o ponto inverso de $P$ quando ele é o único ponto pertencente à reta $\overleftrightarrow{O P}$ tal que d $(O, P) . d\left(O, P^{\prime}\right)=r^{2}$, onde $r$ é o raio do círculo.

Apresentaremos, a seguir, o métodos de construção do ponto inverso $P^{\prime}$, quando $P$ está no interior do círculo e quando $P$ está no seu exterior.

Construção 6 Dado um ponto $P$, no interior de um círculo de centro $O$, obter, por construção com régua e compasso, o ponto inverso $P^{\prime}$.

Para que se obtenha o ponto $P^{\prime}$ procurado, quando $P$ está no interior do círculo, é necessário proceder da seguinte forma:

1 - primeiramente, deve-se traçar a reta $\overleftrightarrow{O P}$

2 - desenhar a reta perpendicular a $\overleftrightarrow{O P}$ que passa por $P$. A referida perpendicular intersectará o círculo em dois pontos, $A$ e $B$;

3 - finalmente, deve-se traçar a reta $t$, tangente ao círculo que passa por $A$ (ou por $B$ ). O ponto $P^{\prime}$ é a interseção de $t \operatorname{com} \overleftrightarrow{O P}$.

Justificativa. Da figura, tem-se que, como $\overline{O A}$ é raio e $t$ é a reta tangente que passa por $A, \overline{O A}$ é perpendicular a $t$. Daí, segue que o triângulo $O A P^{\prime}$ é 


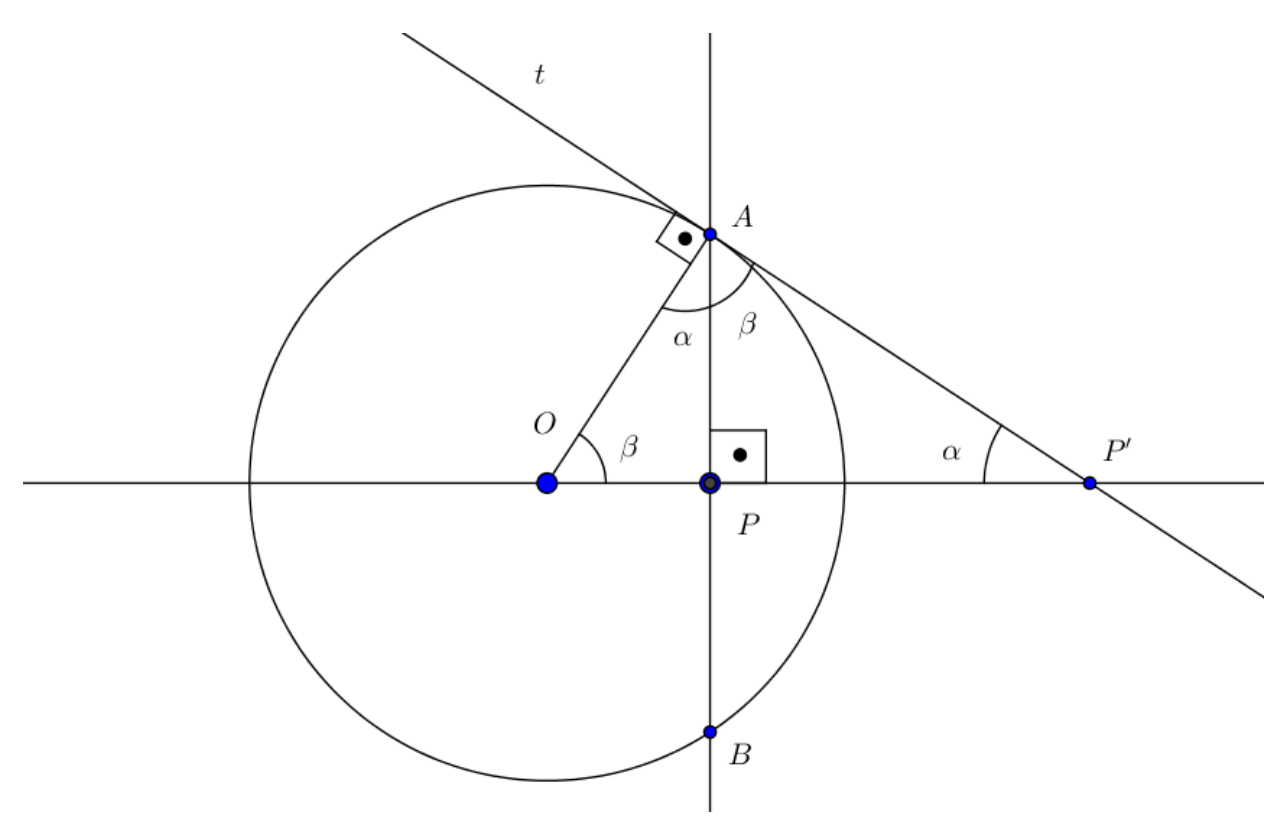

Figura 2.14: Ponto Inverso

retângulo e $\overline{A P}$ é a altura relativa à hipotenusa $\overline{O P^{\prime}}$. Considerando os ângulos marcados na figura, conclui-se que os triângulos $O A P^{\prime}$ e $O P A$ são semelhantes. Logo,

$$
\overline{\overline{O P}}=\frac{\overline{O P^{\prime}}}{\overline{O A}} \Rightarrow \overline{O P} \cdot \overline{O P^{\prime}}=\overline{O A}^{2}
$$

Mas $\overline{O A}=r$, raio do círculo. Deste modo, $d(O, P) \cdot d\left(O, P^{\prime}\right)=r^{2}$ e, de fato, $P^{\prime}$ é o inverso de $P$, como queríamos demonstrar.

É possível observar, portanto, que quando o ponto $P$ está no interior do círculo, o ponto inverso $P^{\prime}$ está no exterior do círculo e vice-versa. Neste contexto, deve ser apresentado o método de obtenção de $P^{\prime}$ quando $P$ está no exterior do círculo.

Construção 7 Considere um círculo de centro $O$ e um ponto $P$, exterior ao círculo. Obter, por construção com régua e compasso, o ponto inverso $P^{\prime}$.

Quando $P$ está fora do círculo, para obter $P^{\prime}$, deve-se traçar o segmento $\overline{O P}$, bem como as retas tangentes ao círculo (construção 5) que passam por $P$ e o intersectam nos pontos $T_{1}$ e $T_{2}$. Em seguida, deve-se traçar o segmento $\overline{T_{1} T_{2}} . P^{\prime}=\overline{T_{1} T_{2}} \cap \overline{O P}$ (figura 2.15).

Outra observação importante é o fato de que, quanto mais próximo de $O$ está o ponto $P$, mais distante se encontra o ponto $P^{\prime}$ e vice-versa, de modo que a inversão para o próprio ponto $O$ não está definida.

É relevante, para o escopo da presente dissertação, estudar as propriedades da inversão circular, de modo que serão enunciados e provados, adiante, os resultados pertinentes quanto a este tópico. 


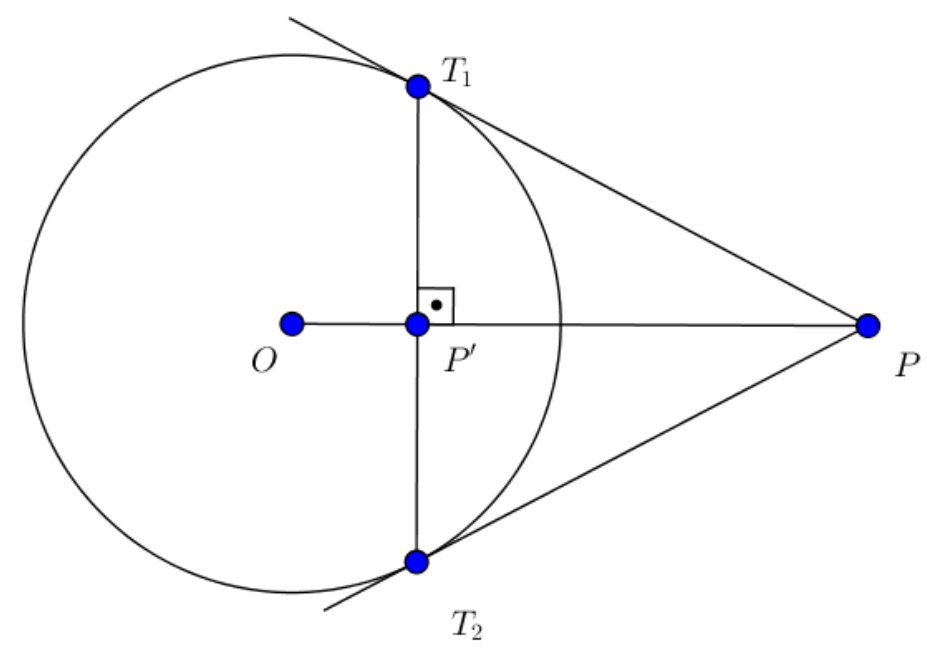

Figura 2.15: Inverso de um ponto exterior ao círculo

Teorema 7 Seja $\Gamma$ um círculo de centro O. Então, a inversão, por $\Gamma$, de uma reta que passa por $O$ é a própria reta. Ainda, uma reta que não passa por $O$ é transformada, pela inversão, num círculo que passa por $O$ e vice-versa.

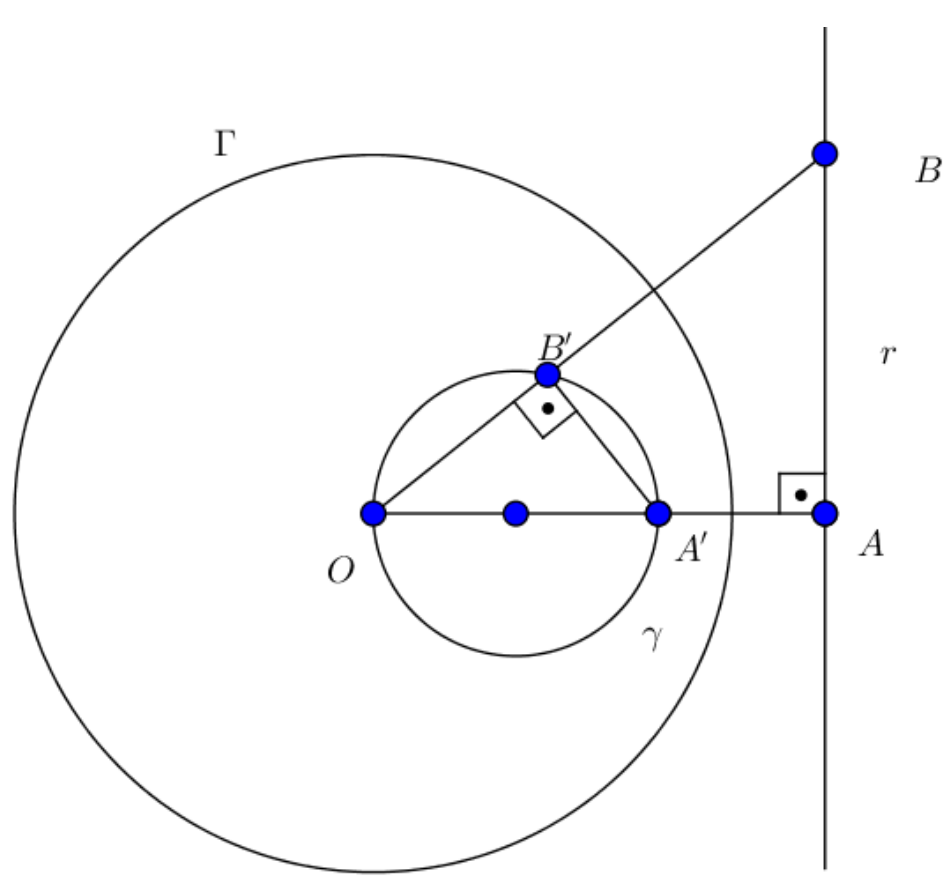

Figura 2.16: Inversão de uma reta

Prova. Primeiramente, note que, de fato, uma reta que passa por $O$ é transformada na própria reta, haja vista que, pela própria definição de inversão circular, conclui-se que os inversos dos pontos da reta estão sobre ela própria. Agora, seja $r$ uma reta que não passa por $O$. Consideramos, na figura 2.16, a hipótese em que $r$ não intersecta $\Gamma$. Os demais casos se provam de modo análogo. 
Trace a perpendicular a $r$ que passa por $O$ e intersecta a referida reta em $A$. Obtendo o ponto inverso $A^{\prime}$ de $A$, desenhe o círculo $\gamma$ de diâmetro $\overline{O A^{\prime}}$. Afirmamos que $\gamma$ é o lugar geométrico dos inversos dos pontos de $r$.

De fato, seja $B$ um outro ponto qualquer de $r$. Traçando o segmento $\overline{O B}$, este encontrará $\gamma$ no ponto $B^{\prime}$. Como $\overline{O A^{\prime}}$ é diâmetro, segue que o triângulo $O B^{\prime} A^{\prime}$ é retângulo em $B^{\prime}$. Mas o triângulo $O A B$ é retângulo em $A$ e, além disso, tem um ângulo em comum com $O B^{\prime} A^{\prime}$, que é $A^{\prime} \hat{O} B^{\prime}=A \hat{O} B$. Logo, $O B^{\prime} A^{\prime} \sim O A B$, donde resulta que

$$
\overline{\overline{O B^{\prime}}}=\frac{\overline{O A}}{\overline{O B}} \Rightarrow \overline{O B} \cdot \overline{O B^{\prime}}=\overline{O A} \cdot \overline{O A^{\prime}}
$$

Como $A^{\prime}$ é o inverso de $A$, segue que $\overline{O A} \cdot \overline{O A^{\prime}}=r^{2}$, em que $r$ é o raio de $\Gamma$. Por conseguinte, $\overline{O B} \cdot \overline{O B^{\prime}}=r^{2}$, o que prova que a inversão transforma a reta $r$ no círculo $\gamma$ (com exceção de $O$ ) e vice-versa.

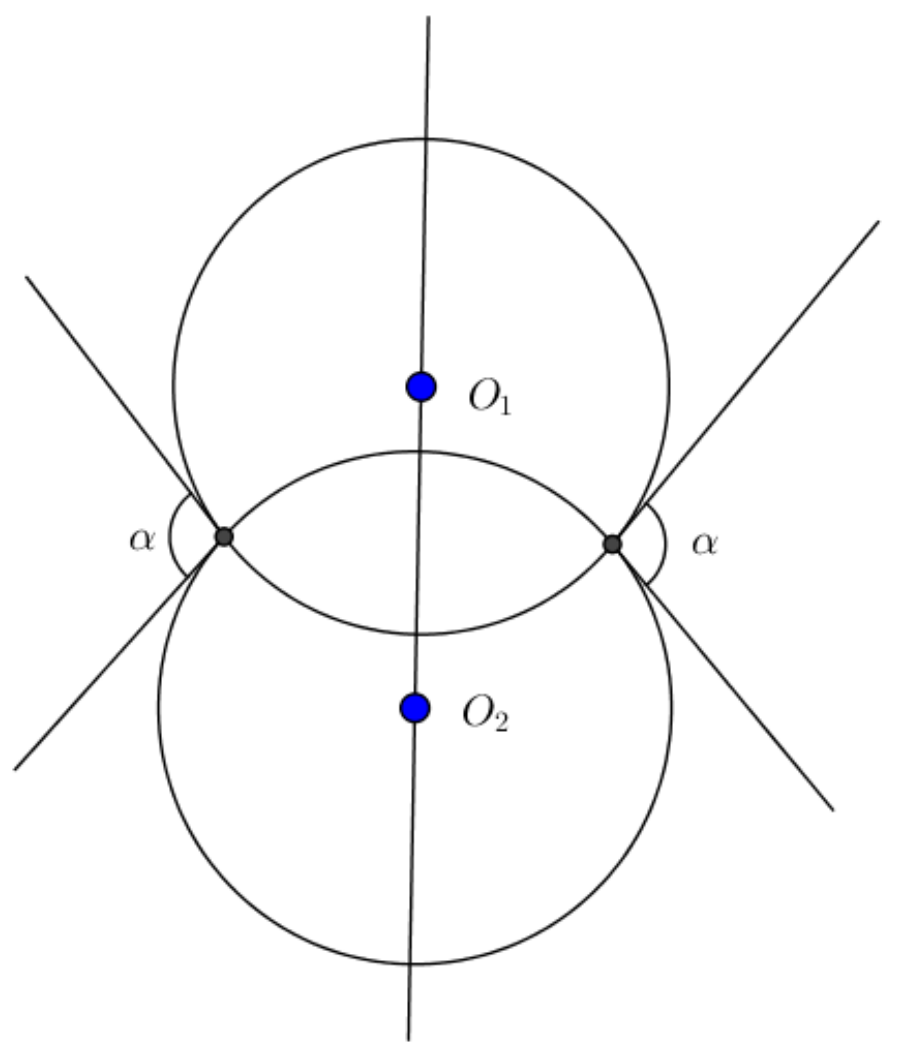

Figura 2.17: Ângulo entre círculos

\section{Definição 15 (Ângulo entre Círculos ou entre Círculo e Reta)}

Quando dois círculos (ou um círculo e uma reta) se intersectam, dizemos que o ângulo entre eles é o ângulo formado entre as retas tangentes nos pontos de contato (ou o ângulo formado entre a reta tangente ao círculo no ponto de interseção e a outra reta dada). 
Deve-se assinalar que, quando dois círculos são secantes, o ângulo entre eles é o mesmo nos dois pontos de contato, uma vez que os dois círculos são simétricos em relação à reta que passa pelos seus centros.

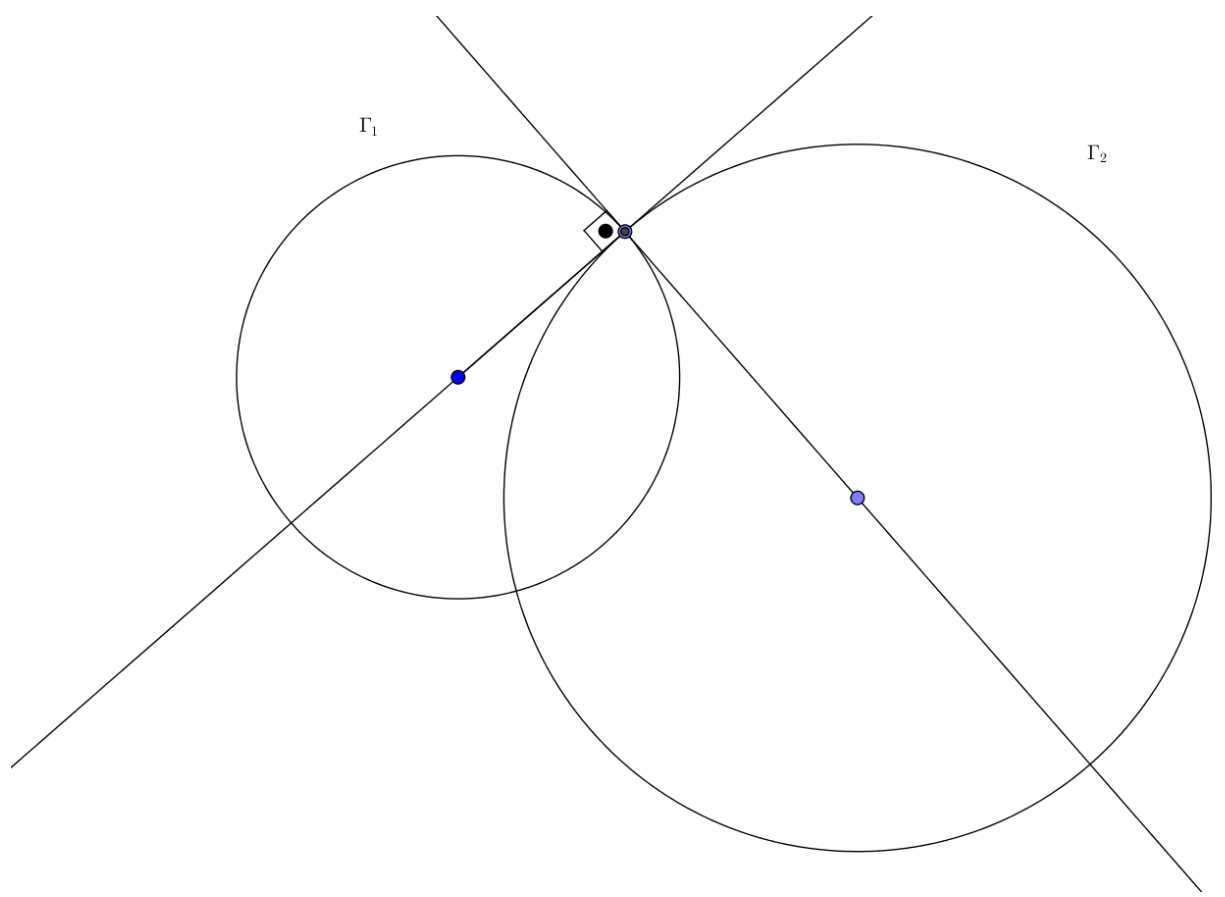

Figura 2.18: Círculos Perpendiculares

A definição de ângulo entre círculos, exposta acima, conduz à definição de círculos perpendiculares, exposta adiante, a qual é necessária para o desenvolvimento de um dos modelos para a Geometria Hiperbólica, devido a Poincaré.

Definição 16 (Círculos Perpendiculares) Sejam $\Gamma_{1}$ e $\Gamma_{2}$ dois círculos secantes. Dizemos que $\Gamma_{1}$ e $\Gamma_{2}$ são perpendiculares quando as retas tangentes aos dois círculos, que passam pelos pontos de contato, são perpendiculares.

Na prática, como o raio de um círculo é perpendicular à reta tangente, no ponto de tangência, tem-se que as retas suportes dos raios de $\Gamma_{1}$ e $\Gamma_{2}$ que têm extremidade nos pontos de contato são perpendiculares (figura 2.18).

A seguir, apresentaremos uma definição e provaremos uma resultado que será útil na demonstração do próximo teorema.

Definição 17 (Ângulo de Segmento) Dado um círculo $\Gamma$ e um ponto $A \in$ $\Gamma$, trace a reta tangente $\overleftrightarrow{A T}$ a $\Gamma$ que passa por $A$ e a corda $\overline{A B}$, em que $B$ é outro ponto qualquer pertencente a $\Gamma$. Neste caso, dizemos que BÂT é um ângulo de segmento (figura 2.19).

Proposição 9 O ângulo de segmento $\alpha$ tem medida igual à metade da do arco subtendido $\widehat{A B}$. 


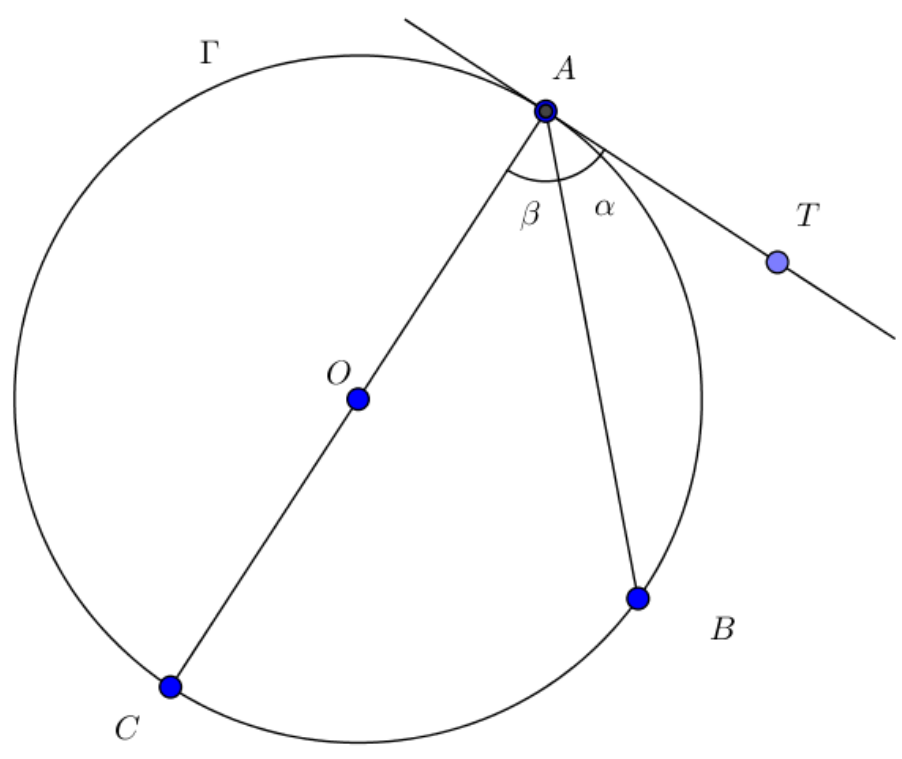

Figura 2.19: Ângulo de Segmento

Prova. Da figura 2.19, percebe-se que, $\alpha+\beta=\frac{\pi}{2}(1)$, tendo em vista que $\overleftrightarrow{A T}$ é tangente a $\Gamma$ e $\overline{O A}$ é raio. Ademais, uma vez que $\overline{A C}$ é diâmetro, tem-se que $\widehat{A B}+\widehat{B C}=\pi(2)$, em que $\widehat{A B}$ e $\widehat{B C}$ estão do mesmo lado de $\overleftrightarrow{A C}$ que $T$. Note-se, também, que $\beta=\frac{\widehat{B C}}{2}$ (ângulo inscrito).

Multiplicando a primeira relação por 2, obtemos:

$$
2 . \alpha+2 . \beta=\pi
$$

Como $\widehat{B C}=2 . \beta$, segue que

$$
2 . \alpha+\widehat{B C}=\pi
$$

Subtraindo (3) de (2), chegamos a:

$$
2 . \alpha-\widehat{A B}=0 \Rightarrow 2 . \alpha=\widehat{A B} \Rightarrow \alpha=\frac{\widehat{A B}}{2}
$$

Teorema 8 Sejam $\Gamma_{1}$ e $\Gamma_{2}$ dois círculos secantes, de centros $O_{1}$ e $O_{2}$, respectivamente. Então, $\Gamma_{1}$ e $\Gamma_{2}$ são perpendiculares se, e somente se, para todo ponto $P \in \Gamma_{2}$, tem-se que $P^{\prime} \in \Gamma_{2}$, em que $P^{\prime}$ é o inverso de $P$ com relação a $\Gamma_{1}$.

Prova. Considere que $\Gamma_{1}$ e $\Gamma_{2}$ são círculos perpendiculares e seja $P$ um ponto qualquer de $\Gamma_{2}$ (figura 2.20). Assim, o raio $\overline{O_{1} A}$ de $\Gamma_{1}$ é tangente a $\Gamma_{2}$ e, por 


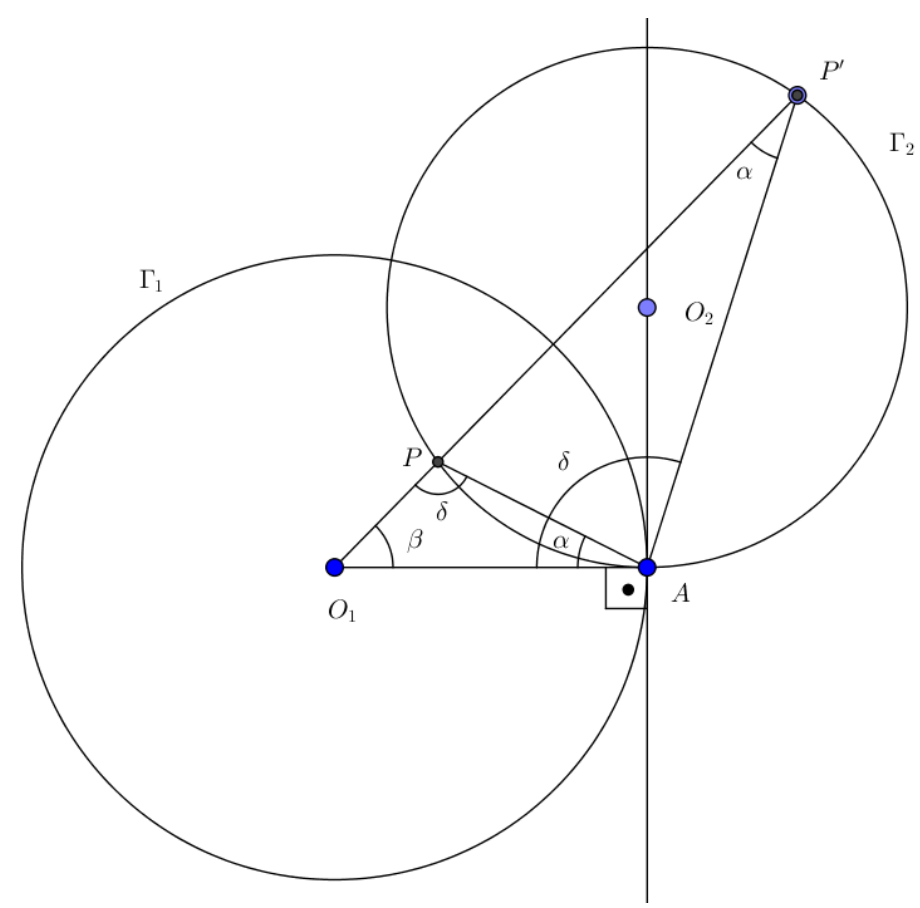

Figura 2.20: Teorema-Círculos Perpendiculares e Pontos Inversos

consequência, o ângulo $O_{1} \hat{A} P$, de segmento em relação a $\Gamma_{2}$, tem medida que corresponde a $\frac{\widehat{A P}}{2}(\widehat{A P}$ é o menor dos dois arcos possíveis $)$.

Observe-se, ainda, que, por ser ângulo inscrito em $\Gamma_{2}, P \hat{P}^{\prime} A$ também mede $\frac{\widehat{A P}}{2}$. Portanto, $O_{1} \hat{A} P=P \hat{P}^{\prime} A=O_{1} \hat{P}^{\prime} A=\alpha$. Além disso, tem-se que $A \hat{O}_{1} P=A \hat{O}_{1} P^{\prime}=\beta$ (ângulo comum). Por conseguinte, os triângulos $O_{1} A P$ e $O_{1} P^{\prime} A$ são semelhantes, o que resulta em

$$
\frac{\overline{O_{1} P}}{\overline{O_{1} A}}=\frac{\overline{O_{1} A}}{\overline{O_{1} P^{\prime}}} \Rightarrow \overline{O_{1} P} \cdot \overline{O_{1} P^{\prime}}={\overline{O_{1} A}}^{2} .
$$

Mas $\overline{O_{1} A}=r_{1}$, raio de $\Gamma_{1}$. Logo, $P^{\prime}$ é o inverso de $P$ com relação a $\Gamma_{1}$ e $P^{\prime} \in \Gamma_{2}$.

Reciprocamente, se $d\left(O_{1}, P\right) \cdot d\left(O_{1}, P^{\prime}\right)=r_{1}^{2}$, com $P^{\prime} \in \Gamma_{2}$, tem-se que:

$$
\overline{O_{1} P} \cdot \overline{O_{1} P^{\prime}}=r_{1}^{2} \Rightarrow \frac{\overline{O_{1} P}}{r_{1}}=\frac{r_{1}}{\overline{O_{1} P^{\prime}}}
$$

Como $r_{1}=\overline{O_{1} A}\left(\right.$ raio de $\left.\Gamma_{1}\right)$, segue que:

$$
\overline{\overline{O_{1} P}}=\overline{\overline{O_{1} A}}
$$

Ademais, tem-se que $A \hat{O}_{1} P=A \hat{O}_{1} P^{\prime}$. Logo, os triângulos $O_{1} A P$ e $O_{1} P^{\prime} A$ tem um ângulo comum e lados adjacentes ao ângulo proporcionais e, portanto, 
são semelhantes. Daí, segue que o ângulo $O_{1} \hat{A} P$ é congruente a $O_{1} \hat{P}^{\prime} A$. Mas $O_{1} \hat{P}^{\prime} A=\frac{\widehat{A P}}{2}$ (menor dos dois arcos possíveis) e, por via de consequência, $O_{1} \hat{A} P=\frac{\widehat{A P}}{2}$. Uma vez que $\overline{O_{1} A}$ não é corda de $\Gamma_{2}$ e $O_{1} \hat{A} P=\frac{\widehat{A P}}{2}$, deve-se concluir, forçosamente, que $O_{1} \hat{A} P$ é um ângulo de segmento e o raio $\overline{O_{1} A}$ é tangente a $\Gamma_{2}$, o que prova a recíproca do teorema.

O teorema provado acima implica que, se $\Gamma_{1}$ e $\Gamma_{2}$ são dois círculos perpendiculares, então a inversão de $\Gamma_{2}$ em relação a $\Gamma_{1}$ é o próprio $\Gamma_{2}$, pois a transformação referida leva pontos de $\Gamma_{2}$ em seus inversos, os quais estão sobre este próprio círculo.

Outro comentário interessante é que toda reta que passa por $O_{1}$, centro de $\Gamma_{1}$ e por um ponto $P \in \Gamma_{2}$ encontrará este último círculo também em $P^{\prime}$, inverso de $P$ com relação a $\Gamma_{1}$.

Teorema 9 Seja $\gamma$ um círculo que não passa pelo centro $O_{\Gamma}$ de outro círculo $\Gamma$. Então $\gamma$ é transformado, por inversão circular em relação a $\Gamma$, em outro círculo $\gamma^{\prime}$.

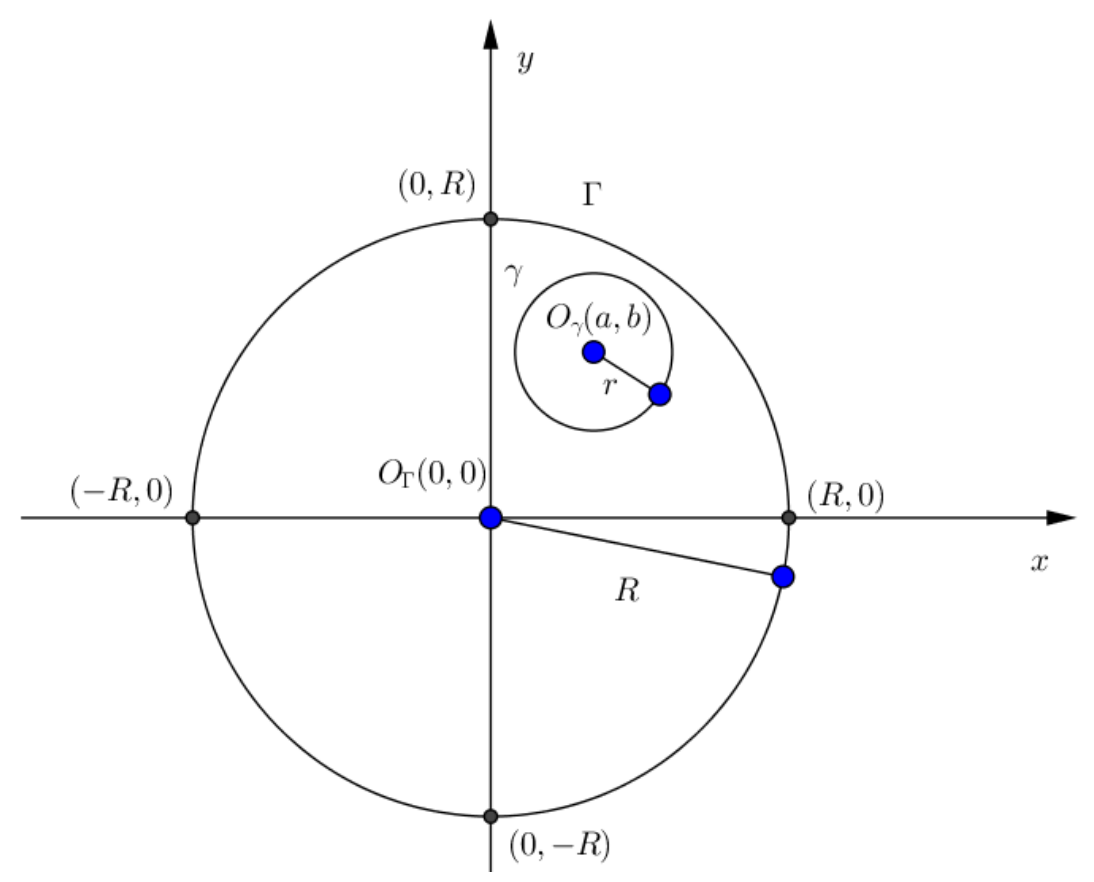

Figura 2.21: Inversão do círculo

Prova. Para tal demonstração, utilizaremos a Geometria Analítica.

Seja $\Gamma$ o círculo pelo qual se pretende fazer a inversão. Posicionando os eixos, de forma conveniente, com origem coincidente com o centro $O_{\Gamma}$, tem-se que a equação de $\Gamma$ é dada por $x^{2}+y^{2}=R^{2}$, em que $R$ é o raio de $\Gamma$. 
Inicialmente, dado um ponto $P=(x, y)$, determinaremos as coordenadas do ponto inverso $\left(x^{\prime}, y^{\prime}\right)$, em relação a $x$ e a $y$.

Pela definição de ponto inverso, tem-se que $d\left(O_{\Gamma}, P\right) \cdot d\left(O_{\Gamma}, P^{\prime}\right)=R^{2}$. Logo,

$$
\sqrt{x^{2}+y^{2}} \cdot \sqrt{\left(x^{\prime}\right)^{2}+\left(y^{\prime}\right)^{2}}=R^{2}
$$

$P$ e o seu inverso pertencem à mesma reta $y=m x$, que passa pela origem.

Daí, segue que

$$
\begin{gathered}
\sqrt{x^{2}+(m x)^{2}} \cdot \sqrt{\left(x^{\prime}\right)^{2}+\left(m x^{\prime}\right)^{2}}=R^{2} \\
\sqrt{\left(1+m^{2}\right) \cdot x^{2}} \cdot \sqrt{\left(1+m^{2}\right) \cdot\left(x^{\prime}\right)^{2}}=R^{2} \\
\left(1+m^{2}\right) \cdot|x| \cdot\left|x^{\prime}\right|=R^{2} \\
\left|x^{\prime}\right|=\frac{R^{2}}{\left(1+m^{2}\right) \cdot|x|}
\end{gathered}
$$

Como $P$ e o seu inverso estão no mesmo quadrante, $x$ e $x^{\prime}$ possuem o mesmo sinal, donde se conclui que

$$
x^{\prime}=\frac{R^{2}}{\left(1+m^{2}\right) \cdot x} \Rightarrow x^{\prime}=\frac{R^{2} \cdot x}{\left(1+m^{2}\right) \cdot x^{2}} \Rightarrow x^{\prime}=\frac{R^{2} \cdot x}{x^{2}+y^{2}}
$$

Por processo análogo, obtém-se $y^{\prime}$ em função de $x$ e $y$ :

$$
\begin{gathered}
\sqrt{\frac{y^{2}}{m^{2}}+y^{2}} \cdot \sqrt{\frac{\left(y^{\prime}\right)^{2}}{m^{2}}+\left(y^{\prime}\right)^{2}}=R^{2} \\
\sqrt{\left(\frac{1+m^{2}}{m^{2}}\right) \cdot y^{2} \cdot \sqrt{\left(\frac{1+m^{2}}{m^{2}}\right) \cdot\left(y^{\prime}\right)^{2}}}=R^{2} \\
y^{\prime}=\frac{R^{2}}{\left(\frac{1+m^{2}}{m^{2}}\right) \cdot y} \Rightarrow y^{\prime}=\frac{R^{2} \cdot y}{\left(\frac{1+m^{2}}{m^{2}}\right) \cdot y^{2}} \Rightarrow y^{\prime}=\frac{R^{2} \cdot y}{x^{2}+y^{2}}
\end{gathered}
$$

Portanto, a inversão $T$ tem equação

$$
T(x, y)=\left(\frac{R^{2} \cdot x}{x^{2}+y^{2}}, \frac{R^{2} \cdot y}{x^{2}+y^{2}}\right)
$$

Considere, agora, um círculo qualquer $\gamma$ de centro $O_{\gamma}=(a, b)$ e raio $r$, que tem equação $(x-a)^{2}+(y-b)^{2}=r^{2}$ e um ponto $P_{I}=\left(x_{I}, y_{I}\right)$, cujo inverso pertence a $\gamma$. Deste modo, o ponto $P=\left(\frac{R^{2} \cdot x_{I}}{x_{I}^{2}+y_{I}^{2}}, \frac{R^{2} \cdot y_{I}}{x_{I}^{2}+y_{I}^{2}}\right)$ satisfaz a equação de $\gamma$. Logo,

$$
\left(\frac{R^{2} \cdot x_{I}}{x_{I}^{2}+y_{I}^{2}}-a\right)^{2}+\left(\frac{R^{2} \cdot y_{I}}{x_{I}^{2}+y_{I}^{2}}-b\right)^{2}=r^{2}
$$


Desenvolvendo e dividindo ambos os membros da equação por $x_{I}^{2}+y_{I}^{2}$, obtemos

$$
\begin{gathered}
R^{4}-2 R^{2} a x_{I}-2 R^{2} b y_{I}+a^{2} \cdot\left(x_{I}^{2}+y_{I}^{2}\right)+b^{2} \cdot\left(x_{I}^{2}+y_{I}^{2}\right)=r^{2} \cdot\left(x_{I}^{2}+y_{I}^{2}\right) \\
\left(a^{2}+b^{2}-r^{2}\right) \cdot x_{I}^{2}-2 R^{2} a x_{I}+\left(a^{2}+b^{2}-r^{2}\right) \cdot y_{I}^{2}-2 R^{2} b y_{I}=-R^{4} \\
x_{I}^{2}-2 \cdot \frac{R^{2} a}{a^{2}+b^{2}-r^{2}} \cdot x_{I}+y_{I}^{2}-2 \cdot \frac{R^{2} b}{a^{2}+b^{2}-r^{2}} \cdot y_{I}=-\frac{R^{4}}{a^{2}+b^{2}-r^{2}}
\end{gathered}
$$

Completando quadrados, chegamos a

$$
\left(x_{I}-\frac{R^{2} \cdot a}{a^{2}+b^{2}-r^{2}}\right)^{2}+\left(y_{I}-\frac{R^{2} \cdot b}{a^{2}+b^{2}-r^{2}}\right)^{2}=\frac{R^{4} \cdot r^{2}}{\left(a^{2}+b^{2}-r^{2}\right)^{2}}
$$

que representa um círculo $\gamma^{\prime}$ de centro $O_{\gamma^{\prime}}=\left(\frac{R^{2} . a}{a^{2}+b^{2}-r^{2}}, \frac{R^{2} . b}{a^{2}+b^{2}-r^{2}}\right)$ e raio $\frac{R^{2} \cdot r}{a^{2}+b^{2}-r^{2}}$.

Como o ponto $P_{I}=\left(x_{I}, y_{I}\right)$ foi considerado de forma arbitrária, segue que todo inverso de ponto $\gamma$ pertence a $\gamma^{\prime}$, o que prova o resultado. A figura acima mostra apenas o caso em que $\gamma$ está no interior de $\Gamma$, mas a demonstração realizada é válida em geral.

Convém esclarecer que o ponto inverso de $O_{\gamma}=(a, b)$ em relação a $\Gamma$ tem coordenadas dadas por $T(a, b)=\left(\frac{R^{2} \cdot a}{a^{2}+b^{2}}, \frac{R^{2} \cdot b}{a^{2}+b^{2}}\right)$. Assim, é possível perceber que, em geral, o centro do círculo inverso $\gamma^{\prime}$, obtido acima, não coincide com o inverso do centro de $\gamma$.

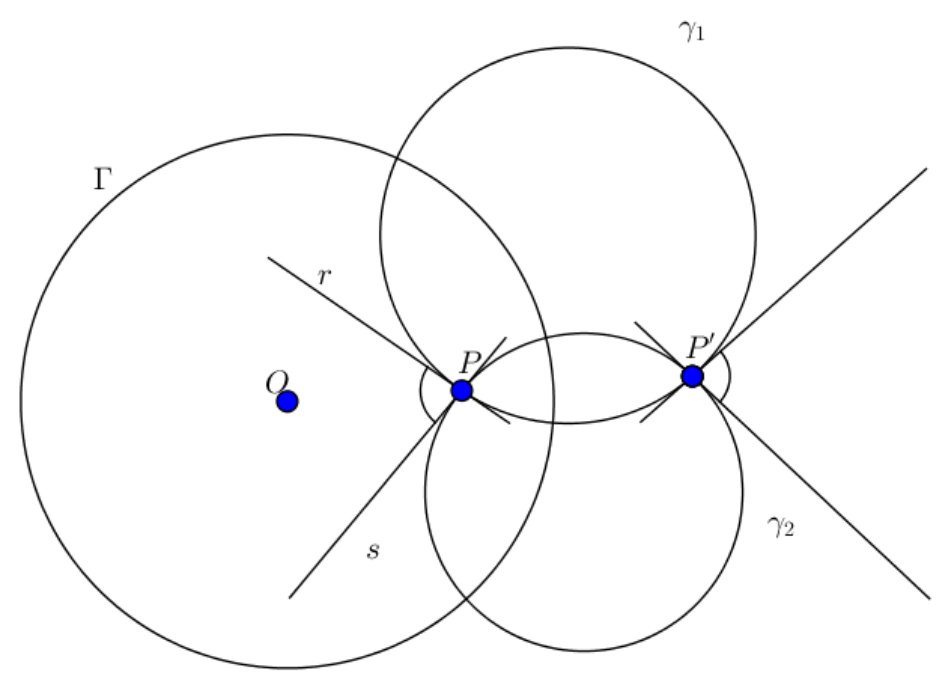

Figura 2.22: Ilustração- inversão conforme 
Teorema 10 A inversão circular é conforme, ou seja, preserva ângulos. Isto significa que, em quaisquer pontos onde duas curvas (círculos ou retas) se intersectam, suas transformações pela inversão circular se encontram com os mesmos ângulos.

Prova. Consideremos, primeiramente, a hipótese em que $P \notin \Gamma$ (figura 2.22). Sejam $r$ e $s$ as tangentes a suas curvas que se intersectam em $P$ (as curvas não aparecem na figura). Então é possível traçar um círculo $\gamma_{1}$ que é tangente a $r$ em $P$ e passa por $P^{\prime}$ (ponto inverso de $\mathrm{P}$ por $\Gamma$ ). Para tanto, deve-se traçar a mediatriz $m$ de $\overline{P P^{\prime}}$ e a perpendicular $t$ a $r$ que passa por P. A interseção de $m$ e $t$ será o centro de $\gamma_{1}$, a partir do qual, com a ponta seca do compasso a outra ponta em $P$ ou $P^{\prime}$, é possível desenhar tal círculo. Por processo análogo, é possível construir $\gamma_{2}$, círculo tangente a $s$ em $P$ e que contém $P^{\prime}$.

O Teorema 8 garante que, como $\gamma_{1}$ e $\gamma_{2}$ passam por $P$ e pelo seu inverso, tais círculos são perpendiculares a $\Gamma$ e, consequentemente, são transformados em si próprios no que diz respeito à inversão circular por $\Gamma$. Logo, as curvas originais (retas ou círculos) serão transformadas em curvas tangentes a $\gamma_{1}$ e a $\gamma_{2}$ e formarão o mesmo ângulo que tinham em $P$, tendo em vista que $\gamma_{1}$ e $\gamma_{2}$ formam o mesmo ângulo nos dois pontos de interseção.

Para o caso em que $P \in \Gamma$, basta observar que $P=P^{\prime}$. Portanto, as retas tangentes às curvas em $P$ e em $P^{\prime}$ são exatamente as mesmas e, assim, formam o mesmo ângulo.

A seguir, será introduzida a definição de razão cruzada. A principal motivação para tal definição diz respeito ao fato de que ela tem relação com com a distância hiperbólica entre dois pontos no modelo do disco de Poincaré, conforme será explicitado no capítulo 3.

Definição 18 Sejam A,B, $P$ e $Q$ quatro pontos distintos no plano. A razão cruzada de tais pontos é definida como

$$
(A B, P Q)=\frac{\frac{d(A, P)}{d(A, Q)}}{\frac{d(B, P)}{d(B, Q)}}
$$

Teorema 11 Dados quatro pontos distintos $A, B, P$ e $Q$, diferentes de $O$, centro do círculo $\Gamma$, tem-se que a inversão pelo círculo referido preserva a razão cruzada, ou seja, se $A^{\prime}, B^{\prime}, P^{\prime}$ e $Q^{\prime}$ são os inversos de $A, B, P$ e $Q$, então

$$
(A B, P Q)=\left(A^{\prime} B^{\prime}, P^{\prime} Q^{\prime}\right)
$$

Prova. Para realizarmos a demonstração deste fato, consideraremos dois casos: 


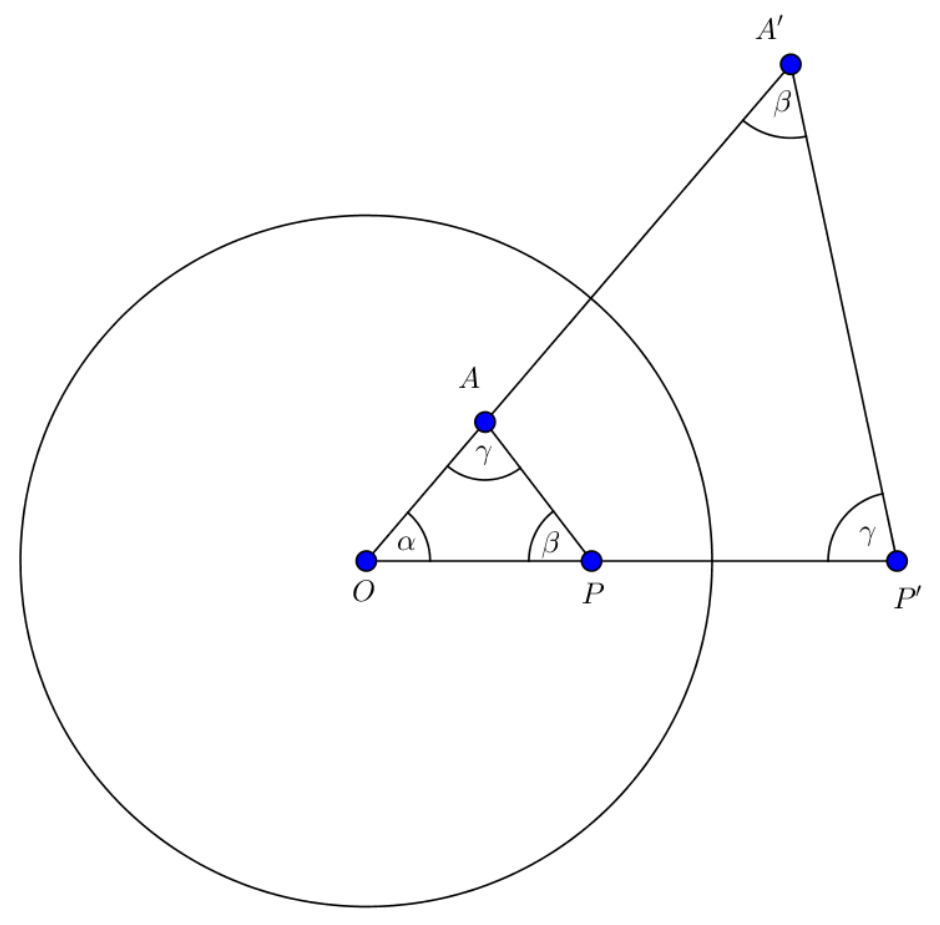

Figura 2.23: $O, A$ e $P$ não colineares.

Caso 1. Os pontos $O, A$ e $P$ não são colineares (figura 2.23).

Nesta hipótese, tem-se que $\overline{O A} \cdot \overline{O A^{\prime}}=r^{2}=\overline{O P} \cdot \overline{O P^{\prime}}$, em que $r$ é o raio do círculo, donde segue que

$$
\overline{\overline{O P}}=\overline{\overline{O P^{\prime}}}
$$

Uma vez que os lados do triângulos $O A P$ e $O P^{\prime} A^{\prime}$ são proporcionais, conforme explicitado acima, e que o ângulo compreendido entre os lados proporcionais $\left(A^{\prime} \hat{O} P^{\prime}=A \hat{O P}=\alpha\right)$ é comum, conclui-se que os referidos triângulos são semelhantes.

Deste modo, podemos obter a seguinte relação:

$$
\frac{\overline{A P}}{\overline{A^{\prime} P^{\prime}}}=\frac{\overline{O A}}{\overline{O P^{\prime}}}
$$

Caso 2. Os pontos $O, A$ e $P$ estão sobre uma mesma reta.

Para este caso, devemos observar o seguinte:

$$
\overline{O A} \cdot \overline{O A^{\prime}}=\overline{O P} \cdot \overline{O P^{\prime}} \Rightarrow \frac{\overline{O A}}{\overline{O P^{\prime}}}=\frac{\overline{O P}}{\overline{O A^{\prime}}}
$$

É conhecida a propriedade das proporções segundo a qual, para quaisquer $x, y, z, w \in \mathbb{R}$, tem-se que $\frac{x}{y}=\frac{z}{w} \Rightarrow \frac{z-x}{w-y}=\frac{x}{y}$. Aplicando a referida 


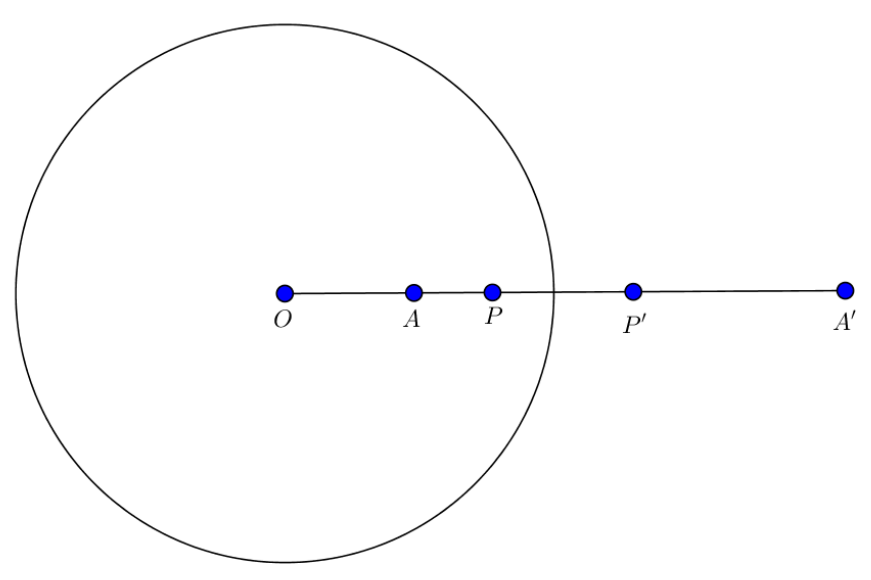

Figura 2.24: Pontos $O, A$ e $P$ sobre uma mesma reta.

propriedade a (2), obtemos

$$
\frac{\overline{O P}-\overline{O A}}{\overline{O A^{\prime}}-\overline{O P^{\prime}}}=\frac{\overline{O A}}{\overline{O P^{\prime}}}
$$

Mas $\overline{O P}-\overline{O A}=\overline{A P}$ e $\overline{O A^{\prime}}-\overline{O P^{\prime}}=\overline{A^{\prime} P^{\prime}}$, de modo que $\frac{\overline{A P}}{\overline{A^{\prime} P^{\prime}}}=\frac{\overline{O A}}{\overline{O P^{\prime}}}$ e a relação (1) mais uma vez se verifica. Ou seja, em qualquer caso se tem (1), donde segue que

$$
\overline{A P}=\frac{\overline{A^{\prime} P^{\prime}} \cdot \overline{O A}}{\overline{O P^{\prime}}}
$$

De modo análogo, considerando outros pontos $B$ e $Q$, tem-se o seguinte:

$$
\begin{aligned}
& \frac{\overline{A Q}}{\overline{A^{\prime} Q^{\prime}}}=\frac{\overline{O A}}{\overline{O Q^{\prime}}} \Rightarrow \overline{A Q}=\frac{\overline{A^{\prime} Q^{\prime} \cdot \overline{O A}}}{\overline{O Q^{\prime}}} \\
& \frac{\overline{B P}}{\overline{B^{\prime} P^{\prime}}}=\frac{\overline{O B}}{\overline{O P^{\prime}}} \Rightarrow \overline{B P}=\frac{\overline{B^{\prime} P^{\prime} \cdot \overline{O B}}}{\overline{O P^{\prime}}} \\
& \frac{\overline{B Q}}{\overline{B^{\prime} Q^{\prime}}}=\frac{\overline{O B}}{\overline{O Q^{\prime}}} \Rightarrow \overline{B Q}=\frac{\overline{B^{\prime} Q^{\prime}} \cdot \overline{O B}}{\overline{O Q^{\prime}}}
\end{aligned}
$$

Considerando as relações (3), (4), (5) e (6), chegamos a

$$
(A B, P Q)=\frac{\overline{\overline{A Q}}}{\frac{\overline{\overline{B P}}}{\overline{\overline{B Q}}}}=\frac{\overline{A P}}{\overline{A Q}} \cdot \frac{\overline{B Q}}{\overline{B P}}=\frac{\frac{\overline{A^{\prime} P^{\prime}} \cdot \overline{O A}}{\overline{O P^{\prime}}}}{\frac{\overline{B^{\prime} Q^{\prime}} \cdot \overline{O B}}{\overline{A^{\prime} Q^{\prime} \cdot \overline{O A}}}} \cdot \frac{\frac{\overline{O Q}}{\overline{B^{\prime} P^{\prime}} \cdot \overline{O B}}}{\overline{O Q^{\prime}}}
$$


Fazendo as simplificações necessárias, obtemos

$$
(A B, P Q)=\frac{\overline{A^{\prime} P^{\prime}}}{\overline{A^{\prime} Q^{\prime}}} \cdot \frac{\overline{B^{\prime} Q^{\prime}}}{\overline{B^{\prime} P^{\prime}}}=\frac{\overline{\overline{A^{\prime} P^{\prime}}}}{\overline{\overline{B^{\prime} Q^{\prime}}}}=\left(A^{\prime} B^{\prime}, P^{\prime} Q^{\prime}\right)
$$




\section{3}

\section{Geometria Hiperbólica}

Desde muito cedo, houve a percepção de que o quinto postulado euclideano, P5, enunciado no começo do Capítulo 2, era mais sofisticado do que os demais e não seria um resultado livre de demonstração; sabe-se que o próprio Euclides tentava evitar a sua utilização na prova de proposições e teoremas.

Vários matemáticos, ao longo dos séculos, tentaram demonstrar o quinto postulado, utilizando-se de $\mathbf{P} \mathbf{1}$ a $\mathbf{P} \mathbf{4}$, acrescidos de um postulado adicional, que, de modo geral, revelava-se equivalente a P5. Empreenderam tentativas de prova Proclus, Clavius, Clairaut, Simson, Playfair,etc. Esta é a primeira fase do desenvolvimento histórico da questão referente ao postulado das paralelas.

A segunda etapa iniciou-se com a conclusão de que $\mathbf{P} 5$ poderia ser negado para que então fossem considerados postulados alternativos, que conduziriam ao nascimento de duas novas geometrias. Os novos postulados seriam os seguintes:

P5 (Hiperbólico). Por um ponto do plano exterior a uma reta, é possivel traçar infinitas retas paralelas a uma reta dada.

P5 (Esférico). Por um ponto do plano exterior a uma reta, não é possivel traçar retas paralelas a uma reta dada.

O nascimento destas novas geometrias foi entrevisto por Gauss, mas seu desenvolvimento, em especial da Geometria Hiperbólica, coube a János (ou Johann) Bolyai e a Nikolai Lobatchevski.

A terceira fase corresponde à prova da consistência destas novas geometrias por meio da utilização de diversos modelos.

O objeto do presente trabalho diz respeito, tão somente, à Geometria Hiperbólica.

\section{1}

\section{A Geometria Hiperbólica. O Modelo do Disco de Poincaré}

A Geometria Hiperbólica é que surge da substituição do postulado 5 euclideano pelo seguinte:

P5(Hiperbólico). Por um ponto do plano exterior a uma reta, é possível traçar infinitas retas paralelas a uma reta dada. 
Todos os demais postulados — P1 a $\mathbf{P 4}$ - são válidos neste nova Geometria, com o acréscimo do postulado 5 modificado. No presente trabalho, as principais noções e resultados da Geometria Hiperbólica serão apresentados com base no modelo do disco de Poincaré.

Modelos são um conjunto de objetos matemáticos postos em correspondência com um dada teoria, com o objetivo de provar a sua consistência, isto é, a ausência de qualquer contradição intrínseca na teoria.

A Geometria Hiperbólica tem diversos modelos que comprovam sua consistência, dentre os quais podem ser citados o de Klein, o da pseudoesfera, devido a Beltrami e o do disco de Poincaré.

No modelo do disco de Poincaré, o plano hiperbólico é representado pelo interior de um círculo euclideano; o bordo ou circunferência deste círculo é chamado de absoluta e representa o infinito, o horizonte. Os pontos da absoluta são chamados de pontos ideais. As retas são representadas por arcos de círculos perpendiculares ao bordo do disco de Poincaré. Nesta dissertação, o disco de Poincaré será apresentado nas figuras de forma destacada, com preenchimento em azul.

As retas que passam pelo centro do disco de Poincaré, são, na verdade, retas euclideanas (e-retas), pois a inversão para o centro do disco não está definida, pois conforme um ponto se aproxima do centro, maior a distância até o respectivo inverso. Por este motivo, é possível pensar em tais retas como "círculos de raio infinito".

$\mathrm{Na}$ sequência, apresentaremos as definições de retas paralelas, nãosecantes ou divergentes e concorrentes. Cabe assinalar que a terminologia aqui adotada nem de longe é unânime entre os autores que tratam do estudo da Geometria Hiperbólica, de modo que podem ser encontradas outras denominações nos diversos textos que abordam este tema.

Definição 19 (Retas Paralelas) Duas retas no disco de Poincaré são ditas paralelas quando são representadas por arcos de círculos perpendiculares à absoluta que passam por um mesmo ponto ideal.

Definição 20 (Retas Não-Secantes ou Divergentes) As demais retas que não se intersectam e não passam por um mesmo ponto ideal são denominadas não secantes ou divergentes.

Definição 21 (Retas Concorrentes) Duas retas são concorrentes no mesmo sentido da Geometria Tradicional, ou seja, quando têm apenas um ponto em comum.

Na figura abaixo, tem-se o seguinte: 


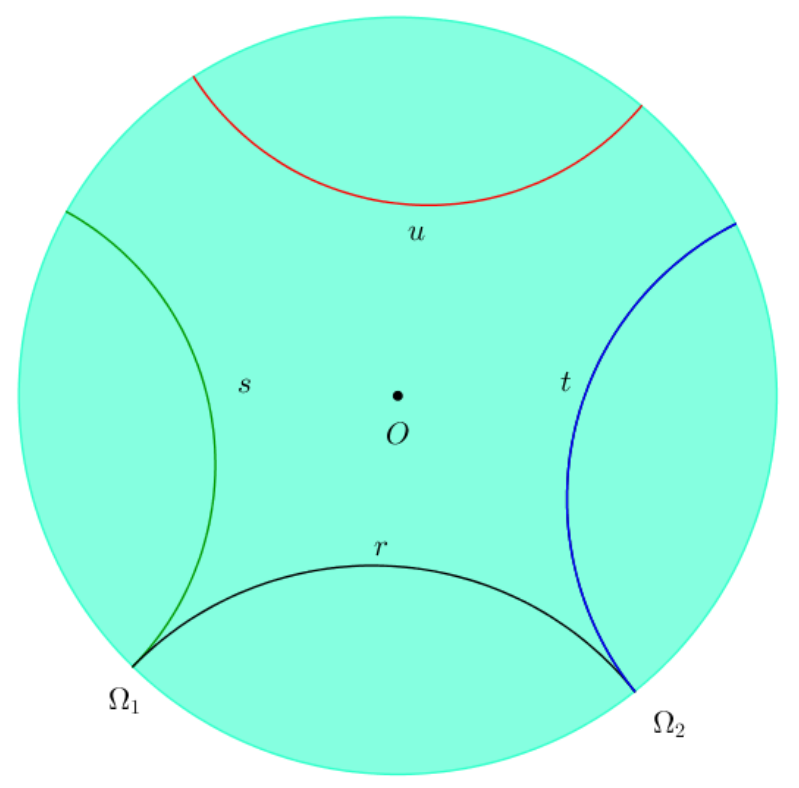

Figura 3.1: Retas paralelas e não-secantes no modelo de Poincaré

$1-r$ e $s$ são paralelas;

$2-r$ e $t$ são paralelas;

$3-s$ e $t$ são não-secantes ou divergentes;

4 - $r$ e $u$ são não-secantes ou divergentes;

$5-s$ e $u$ são não-secantes ou divergentes;

$6-t$ e $u$ são não-secantes ou divergentes.

Tendo em vista que há dois pontos ideais para cada reta — já que os arcos de círculos que representam as retas e a absoluta são secantes —, é possível traçar duas retas paralelas passando por um ponto dado, exterior à reta. As demais que não se encontram — denominadas não-secantes ou divergentes não têm grande relevância para o desenvolvimento do modelo.

É relevante fazer um comentário sobre terminologia: nas construções que serão realizadas adiante, será necessário utilizar algumas retas euclideanas, que serão denominadas e-retas para evitar qualquer possível confusão com as retas hiperbólicas.

\subsection{1}

\section{A Régua e o Compasso Hiperbólicos}

Nesta subseção, primeiramente, provaremos que a reta definida no modelo de Poincaré satisfaz o postulado 1 de Euclides.

Proposição 10 A reta definida para o modelo de Poincaré satisfaz o postulado 1 de Euclides. 
Prova. Para provarmos que a definição de reta satisfaz o postulado 1 de Euclides - uma reta pode ser traçada de um ponto a outro, escolhidos à vontade -, construiremos a reta hiperbólica que passa por dois pontos, digamos $A$ e $B$.

Neste âmbito, devemos lembrar o Teorema 8, provado no Capítulo 2. Como o arco de círculo que representa a reta hiperbólica é perpendicular ao bordo disco de Poincaré, então ele passará pelos pontos $A^{\prime}$ e $B^{\prime}$, inversos de $A$ e $B$ em relação ao bordo do disco. Por conseguinte, deve-se proceder da seguinte forma:

1 - obter $A^{\prime}$ (ou $B^{\prime}$ ), por meio da construção 6 ;

2 - traçar as e-retas mediatrizes $\overleftrightarrow{A B}$ e $\overleftrightarrow{A A^{\prime}}$ ( ou $\overleftrightarrow{B B^{\prime}}$ ). As e-retas referidas intersectar-se-ão em um ponto, que é o centro do $O_{r}$ do arco que representa a reta procurada;

3 - com a ponta seca do compasso em $O_{r}$ e tomando como raio $\overline{O_{r} A}$ ou $\overline{O_{r} B}$, pode-se finalmente traçar a reta hiperbólica que passa por $A$ e $B$.

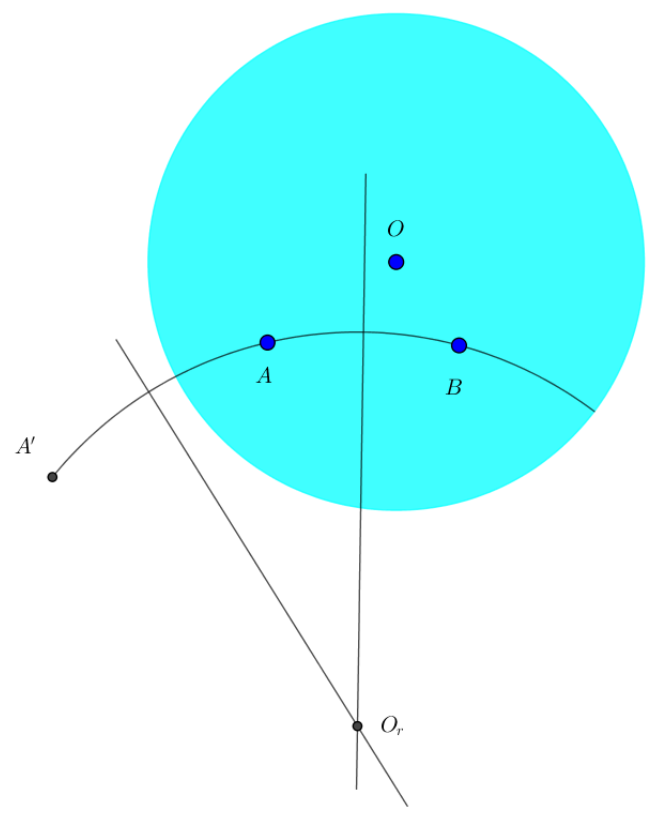

Figura 3.2: Disco de Poincaré — reta hiperbólica por dois pontos

Deste modo, verificamos que existe uma reta hiperbólica, no modelo do disco de Poincaré, que passa por $A$ e $B$.

Para provar que a reta que passa por $A$ e $B$ é única, basta observar que existem infinitos círculos euclideanos que passam por $A$ e $B$, mas nem todos são perpendiculares à absoluta.

Assim, suponhamos que exista outro arco de círculo, passando por $A$ e $B$, que corresponda à definição de reta hiperbólica. Por conseguinte, tal arco será perpendicular à absoluta e, tendo em vista o teorema 8 , ele passará por $A^{\prime}$ e 
$B^{\prime}$, inversos de $A$ e $B$ em relação ao bordo do disco. Ou seja, o referido arco passará por $A, B, A^{\prime}$ e $B^{\prime}$, do mesmo modo que o arco de círculo que contém a reta hiperbólica que passa por $A$ e $B$, donde se conclui que tal arco coincide com a referida reta hiperbólica.

É relevante destacar que, além de a demonstração acima comprovar que dois pontos determinam uma reta na Geometria Hiperbólica (postulado 1), o Teorema 8 garante a existência de uma régua para o disco de Poincaré, que pode ser pensada como uma combinação da régua e do compasso até então utilizados para a Geometria Euclideana.

Neste contexto, é importante também apresentar a construção das retas paralelas a uma reta dada, por um ponto exterior.

Construção 8 Construir, no modelo do disco de Poincaré, duas retas paralelas a uma reta $r$ dada, passando por um ponto exterior $P$.

Para realizar a referida construção, devemos obter, utilizando régua e compasso, os arcos de círculos que passam por $P, \Omega_{1}$ (ponto ideal de $r$ ) e $P^{\prime}$ (ponto inverso de $\mathrm{P}$ com relação ao disco de Poincaré) e por $P, P^{\prime}$ e $\Omega_{2}$ (outro ponto ideal de $r$ ). O processo é semelhante ao descrito acima:

1 - traçar as e-retas mediatrizes de dois pares de pontos dos arcos de círculos euclideanos que representam cada reta, para obter o centro de cada círculo;

2 - com a ponta seca do compasso no centro obtido e a outra ponta em um dos pontos da reta hiperbólica procurada, traçar o arco de círculo que representa a reta.

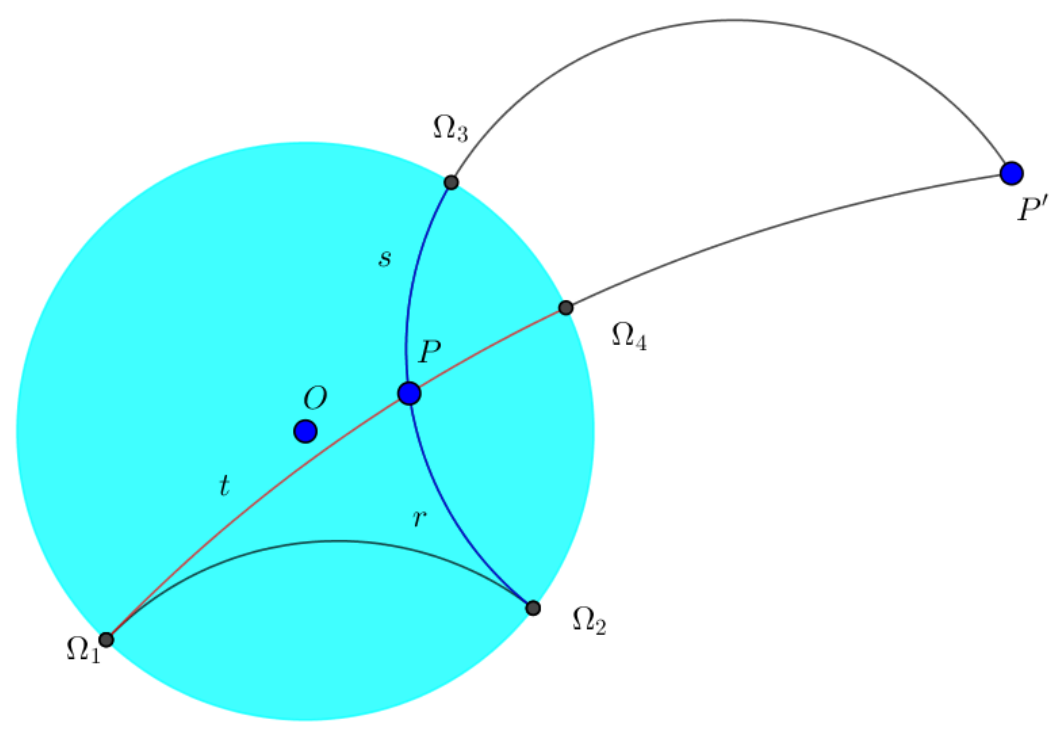

Figura 3.3: Disco de Poincaré - paralelas por ponto dado 
No mesmo espírito do empreendido, provaremos, a seguir, que a reta hiperbólica definida no modelo do disco de Poincaré satisfaz o postulado 2 de Euclides.

Proposição 11 A definição de reta hiperbólica, no modelo do disco de Poincaré, satisfaz o postulado 2 de Euclides.

Prova. Para verificar a validade do postulado 2 no modelo do disco Poincaré, basta observar que a definição de reta como arco de círculo perpendicular ao bordo do disco de Poincaré faz com que o círculo que contém a reta hiperbólica tenha dois pontos de interseção com a absoluta, que representa o infinito ou o horizonte. Deste modo, é claro que a definição de reta do modelo do disco de Poincaré atende ao postulado 2 de Euclides.

Os resultados sobre inversão circular, desenvolvidos no Capítulo 2, permitem estabelecer um método de construção de círculos no modelo de Poincaré, de modo que, adiante, será provado que o modelo do disco de Poincaré também satisfaz o postulado 3 de Euclides (um círculo pode ser traçado com centro e raio arbitrários).

Convém, primeiramente, definir a distância entre pontos no modelo ora estudado.

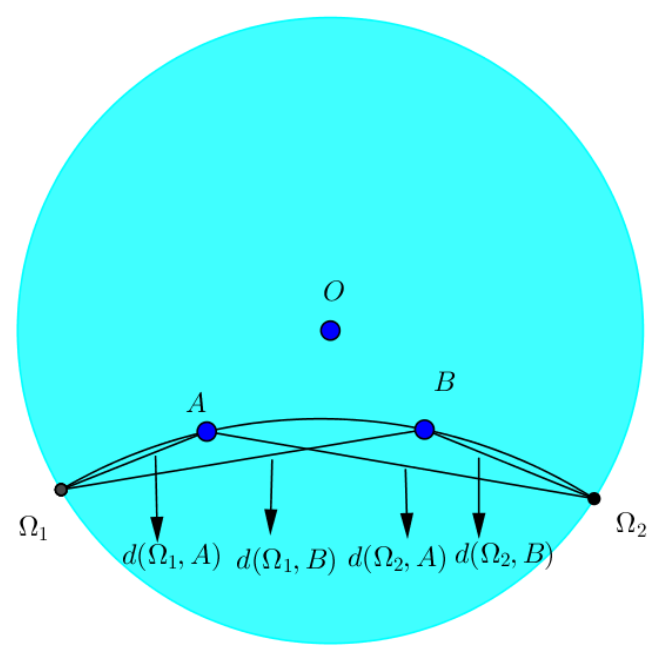

Figura 3.4: Distância no disco de Poincaré

Definição 22 A distância entre dois pontos $A$ e $B$, no modelo do disco de Poincaré, é dada por

$$
d_{h}(A, B)=k \cdot\left|\ln \left[\left(A B, \Omega_{1} \Omega_{2}\right)\right]\right|
$$

em que $\left(A B, \Omega_{1} \Omega_{2}\right)$ é a razão cruzada entre os pontos $A, B, \Omega_{1} e \Omega_{2}$, que envolve as distâncias euclideanas $d\left(\Omega_{1}, A\right), d\left(\Omega_{2}, A\right), d\left(\Omega_{1}, B\right)$ e $d\left(\Omega_{2}, B\right)$ 
(figura 3.4), e onde $k>0$ é uma constante arbitrária, denominada constante de Gauss.

Para entender os motivos de a distância ter sido definida na forma acima, devemos lembrar que, pelos teoremas de inversão circular, não há preservação de comprimentos euclideanos; no que respeito a medidas de segmentos, apenas a razão cruzada é invariante pela inversão circular, conforme se verifica do Teorema 11. Por este motivo, a razão cruzada aparece na definição acima.

Deve-se levar em conta, porém, que uma boa definição de distância deve ser aditiva, no seguinte sentido: dados três pontos $A, B$ e $C$, sobre a mesma reta hiperbólica, com $B$ entre $A$ e $C$, devemos ter $d_{h}(A, C)=$ $d_{h}(A, B)+d_{h}(B, C)$, do mesmo modo que se dá com três pontos colineares sobre uma reta euclideana.

No entanto, a razão cruzada não é aditiva e sim multiplicativa. Note-se que

$$
\left(A B, \Omega_{1} \Omega_{2}\right) \cdot\left(B C, \Omega_{1} \Omega_{2}\right)=\frac{\frac{d\left(\Omega_{1}, A\right)}{d\left(\Omega_{2}, A\right)}}{\frac{d\left(\Omega_{1}, B\right)}{d\left(\Omega_{2}, B\right)}} \frac{\frac{d\left(\Omega_{1}, B\right)}{d\left(\Omega_{2}, B\right)}}{\frac{d\left(\Omega_{1}, C\right)}{d\left(\Omega_{2}, C\right)}}=\frac{\frac{d\left(\Omega_{1}, A\right)}{d\left(\Omega_{2}, A\right)}}{\frac{d\left(\Omega_{1}, C\right)}{d\left(\Omega_{2}, C\right)}}=\left(A C, \Omega_{1} \Omega_{2}\right)
$$

Portanto, a princípio, a razão cruzada não seria uma boa definição de distância, já que é multiplicativa e não aditiva. No entanto, sabemos que a função logaritmo (ln) transforma produto em soma. Assim, aplicando ln à razão cruzada, na forma acima, obtemos uma definição de distância com a característica aditiva pretendida.

O módulo utilizado na expressão acima é apenas para garantir que a distância definida seja sempre positiva. É interessante esclarecer que, do mesmo que na Geometria Tradicional, a distância de um ponto $A$ a ele próprio é 0 , pois:

$$
d_{h}(A, A)=k \cdot\left|\ln \frac{\frac{d\left(\Omega_{1}, A\right)}{d\left(\Omega_{2}, A\right)}}{\frac{d\left(\Omega_{1}, A\right)}{d\left(\Omega_{2}, A\right)}}\right|=k \cdot \ln 1=0
$$

Voltando aos pormenores relativos à construção do círculo no modelo do disco de Poincaré, enunciaremos e provaremos uma proposição que terá relevância no teorema a ser demonstrado em seguida.

Proposição 12 Dado um ponto A qualquer no disco de Poincaré, existe uma inversão $T$, por um círculo $\Gamma$, perpendicular ao bordo do disco, que leva $A$ ao centro $O$ do disco de Poincaré. 


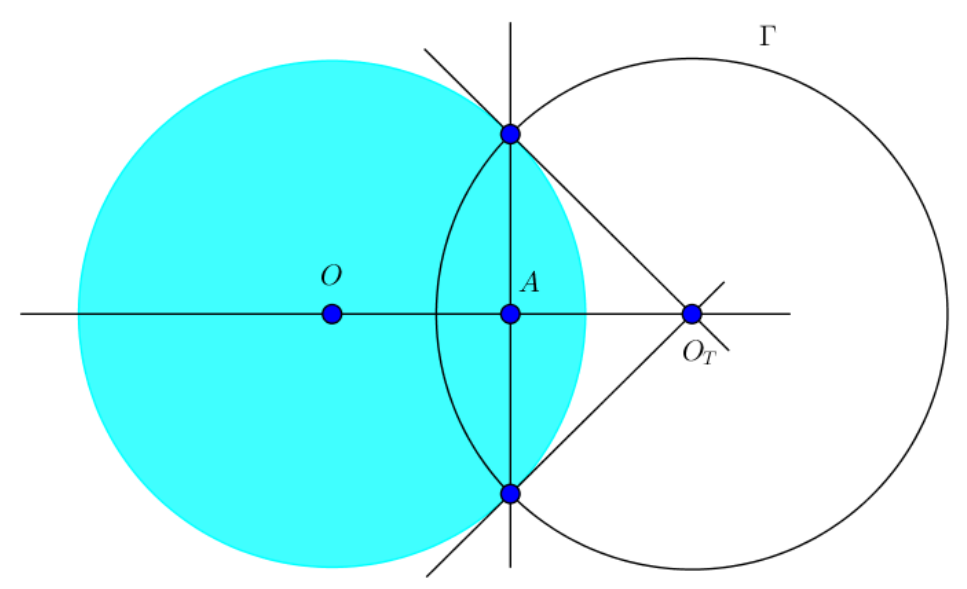

Figura 3.5: Inversão por um círculo perpendicular ao bordo do disco de Poincaré

Prova. Para provarmos a proposição acima, realizaremos uma construção com régua e compasso para obter o círculo procurado, adotando os seguintes passos: 1 - traçar a reta $\overleftrightarrow{O A}$, que conterá o centro de $\Gamma$

2 - traçar a e-reta perpendicular a $\overleftrightarrow{O A}$ passando por $A$, que intersectará o bordo do disco (absoluta) em dois pontos ( $T_{1}$ e $T_{2}$, não nomeados na figura). 3 - desenhar uma das retas tangentes à absoluta, por $T_{1}$ ou $T_{2}$. A interseção das das tangentes com $\overleftrightarrow{O A}$ é o centro $O_{T}$ de $\Gamma$, a partir do qual pode ser desenhado o círculo, que terá raio com outra extremidade no ponto a partir do qual foi construída a tangente (figura 3.5).

É fácil de ver que, se traçarmos o raio euclideano $\overline{O T_{1}}$ da absoluta, o triângulo $O T_{1} O_{\Gamma}$ será retângulo. Da semelhança entre os triângulos $O T_{1} O_{\Gamma}$ e $T_{1} A O_{\Gamma}$, obtemos a relação $\overline{O_{\Gamma} O} \cdot \overline{O_{\Gamma} A}={\overline{O_{\Gamma} T_{1}}}^{2}$, o que prova que $O$ é o inverso de $A$ com relação a $\Gamma$.

Teorema 12 Um círculo hiperbólico, no modelo de Poincaré, é um círculo euclideano contido no disco de Poincaré (mas o seu centro hiperbólico $H$, em geral, não coincide com o seu centro euclideano E).

Prova. Primeiramente, devemos observar que um círculo hiperbólico, centrado em $O$, centro do disco de Poincaré, nada mais é do que um círculo euclideano, uma vez que as retas hiperbólicas que passam por $O$ são também e-retas, de modo que os raios do círculo são segmentos de retas euclideanas. Assim, o lugar geométrico dos pontos equidistantes de $O$, com os raios dados, é a mesma figura já conhecida.

Considere agora um círculo qualquer $\gamma$ no interior do disco de Poincaré, com centro hiperbólico $H$ não coincidente com $O$, centro da absoluta. Afirmamos que $\gamma$ é um círculo euclideano. 
De fato, seja $T$ a inversão por um círculo $\Gamma$, perpendicular ao bordo do disco de Poincaré, que leva $H$ em $O$. Já vimos, no Teorema 11, que a inversão circular preserva a razão cruzada e, consequentemente, a distância hiperbólica. Assim, se aplicarmos $T$ a $\gamma$, obteremos um conjunto de pontos equidistantes hiperbolicamente de $O$, que, pela observação acima, nada mais é do que um círculo euclideano $\gamma^{\prime}$.

$\gamma^{\prime}$ é um círculo euclideano que não passa pelo centro de $\Gamma\left(O_{\Gamma}\right)$ Para verificar este fato, basta observar que $\gamma^{\prime}$ é um círculo no interior do disco de Poincaré e na construção vista na prova da proposição 12 , o ponto $O_{\Gamma}$ está no exterior da absoluta. Deste modo, pelo Teorema $9, T\left(\gamma^{\prime}\right)$ também é um círculo euclideano, ou seja $\gamma$ é um círculo euclideano, como queríamos demonstrar.

Observe-se que, na inversão $T$ pelo círculo $\Gamma$, tem-se que o centro hiperbólico $H$ de $\gamma$ coincide com $O^{\prime}$, ponto inverso de $O$. Por conseguinte, convém recordar que, conforme explicitado na demonstração do Teorema 9, o centro do círculo inverso não coincide com o ponto inverso do centro do círculo original. Por este motivo, o centro hiperbólico de $\gamma^{\prime}\left(O^{\prime}=H\right)$ não é necessariamente o seu centro euclideano $(E)$. A única exceção é quando o círculo é construído com centro do disco de Poincaré, em que $O, E$ e $H$ se confundem.

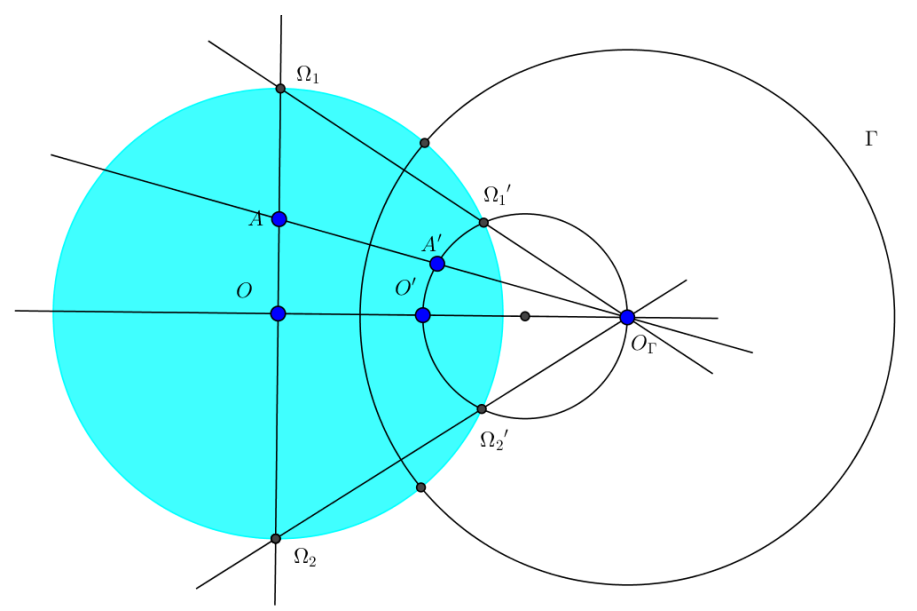

Figura 3.6: Raio do Círculo Hiperbólico

Após a referida demonstração é interessante apontar uma fato que também terá importância para a construção do círculo no modelo do disco de Poincaré: os raios do círculo hiperbólico são perpendiculares ao círculo. Para verificar este fato, basta observar que os raios de um círculo euclideano são perpendiculares ao círculo, já que formam ângulo $\frac{\pi}{2}$ com as retas tangentes nos pontos respectivos pontos de tangência.

Deste modo, os raios do círculo $\gamma^{\prime}$, centrado em $O$ são levados, pela inversão $T$, por $\Gamma$, em arcos de círculo que representam segmentos de retas hiperbólicas (demonstração do teorema 12). Assim, como a inversão é conforme 
(Teorema 10) e $T\left(\gamma^{\prime}\right)=\gamma$, segue que os raios hiperbólicos são perpendiculares ao círculo $\gamma$. A figura 3.6 ilustra a inversão dos raios dos círculos $\gamma$ e $\gamma^{\prime}$.

O teorema acima provado garante que o centro euclideano $(E)$ e o centro hiperbólico $(H)$ de um círculo no disco de Poincaré são diferentes, mas não explicita como obter $E$, o que é fundamental para realizar a construção do círculo. Por este motivo, é necessário, ainda, o resultado que será provado a seguir.

Teorema 13 O centro do disco de Poincaré $(O)$, o centro euclideano de um círculo no referido disco (E) e o centro hiperbólico (H) deste mesmo círculo estão sobre uma mesma reta.

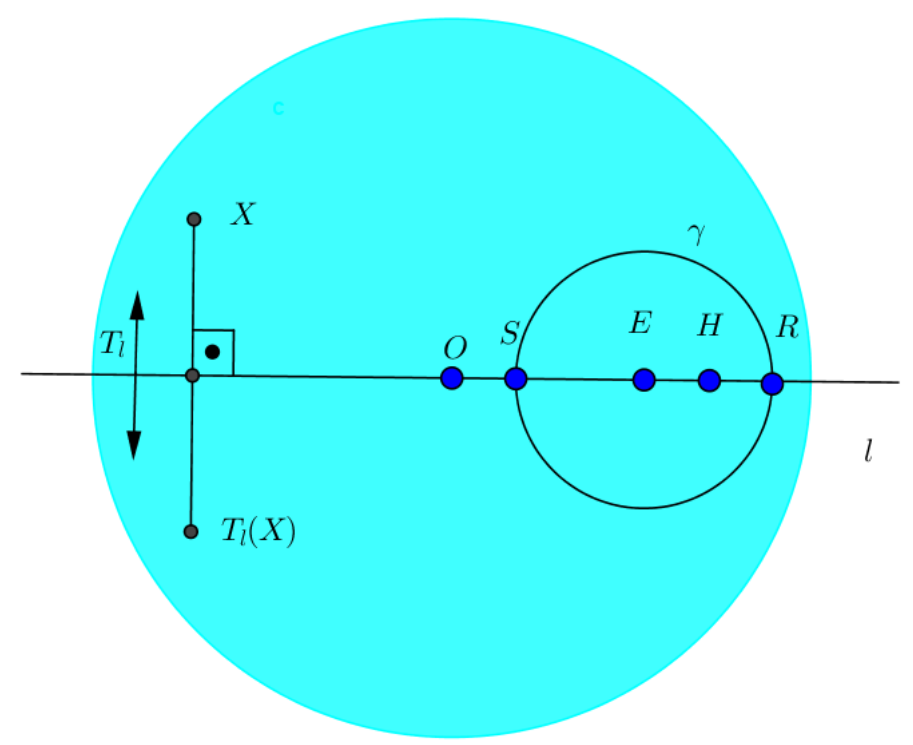

Figura 3.7: $O, E$ e $H$ sobre a mesma reta

Prova. Sejam $l$ a e-reta (também reta hiperbólica) que passa por $O$, centro do disco de Poincaré e $E$, centro euclideano do círculo $\gamma$, mostrado na figura acima. Pela continuidade da distância hiperbólica e da reta, conclui-se que existe um único ponto $H$ em $l$, no diâmetro $\overline{R S}$ tal que $d_{h}(H, R)=d_{h}(H, S)$, em que $d_{h}$ é a distância hiperbólica entre os pontos. Afirmamos que $H$ é o centro hiperbólico de $\gamma$.

Suponha, por absurdo, que o centro hiperbólico de $\gamma$ não esteja em $l$. Considere a reflexão $T_{l}$ pela reta hiperbólica $l$.

Os pontos que estão sobre $l$ são levados em si próprios por $T_{l}$, enquanto os demais pontos são levados em pontos à mesma distância de $l$, em lados opostos da reta e os semicírculos superior e inferior (com relação ao diâmetro $\overline{R S}$ ) de $\gamma$ são levados um no outro.

Assim, como $H$ não está sobre $l$, segue que há um ponto outro ponto $T_{l}(H)$, contido no interior do círculo $\gamma$, obtido pela reflexão de $H$ em relação a $l$. A 
reflexão mencionada tem as mesmas propriedades da inversão circular, dentre as quais a preservação da razão cruzada (teorema 11) e, consequentemente, da distância hiperbólica. Logo, como $H$ é equidistante dos pontos de $\gamma$ e o semicírculo superior é levado no semicírculo inferior por $T_{l}$, segue que $T_{l}(H)$ também é equidistante dos pontos de $\gamma$. Ou seja, $\gamma$ teria dois centros $H$ e $T_{l}(H)$, o que é absurdo.

Para verificar que $\gamma$ não pode ter dois centros, suponhamos que exista um diâmetro $\overline{A B}$, que passa por $H$ e $T_{l}(H)$. Deste modo, tem-se que, se $r$ é o raio de $\gamma$, então $d_{h}(A, B)=2 r$. Mas, $d_{h}(H, A)=d_{h}\left(T_{l}(H), B\right)=r$, donde segue que $d_{h}\left(H, T_{l}(H)\right)=0$ (contradição).

Logo, $H$ está sobre a reta que passa por $O$ e $E$.

De posse de todos os resultados expostos acima, podemos, finalmente, construir o círculo hiperbólico em $H$ no modelo do disco de Poincaré.

Construção 9 Dados o centro hiperbólico $H$ e o ponto $R$, extremidade do raio, construir um círculo hiperbólico no modelo do disco de Poincaré.

Consoante os teoremas acima provados, a construção deve ser realizada da seguinte forma: $\overleftrightarrow{O H}$

1 - primeiramente, deve-se traçar a reta hiperbólica (também e-reta)

2 - em seguida, deve-se desenhar a reta hiperbólica que passa pelos pontos $H$ e $R$, representada por um círculo de centro $Z$;

3 - deve-se traçar o raio $\overline{Z R}$ do círculo que contém a reta hiperbólica que passa por $H$ e $R$;

4 - a partir de $R$, deve-se traçar a e-reta tangente ao e-círculo que passa por $R$ e $H$, perpendicular ao raio $\overline{Z R}$. A referida e-reta encontrará $\overleftarrow{O H}$ no ponto $E$, que é o centro euclideano do círculo que se pretende construir;

5 - com a ponta seca do compasso em $E$ e a outra ponta em $R$, pode-se, então, traçar o círculo hiperbólico procurado.

Justificativa. Como visto após a prova do Teorema 12, as retas hiperbólicas que contém os raios são perpendiculares ao círculo a ser construído, razão pela qual foi traçada a e-reta perpendicular ao raio $\overline{Z R}$ do círculo que contém a reta hiperbólica que passa por $H$ e $R$, a qual é tangente a tal círculo em $R$. Como a circunferência que representa a reta hiperbólica $\overleftrightarrow{H R}$ é perpendicular ao círculo que se pretende traçar, conclui-se que o centro euclideano $E$ do círculo está sobre a e-reta tangente que passa por $R$, mencionada acima, já que este ponto pertence ao círculo.

Ainda, pelo Teorema 13, o ponto $E$ está sobre a reta que passa por $O$ e $H$, donde se conclui que o centro euclideano do círculo é a interseção entre $\overleftarrow{O H}$ e a e-reta tangente que passa por $R$. 
Deste modo, observa-se que o compasso, no modelo do disco de Poincaré, da mesma forma que a régua, pode ser pensado como uma combinação da régua e do compasso tradicionais, pois estes instrumentos, utilizados da forma apropriada, possibilitam a construção do círculo hiperbólico neste modelo.

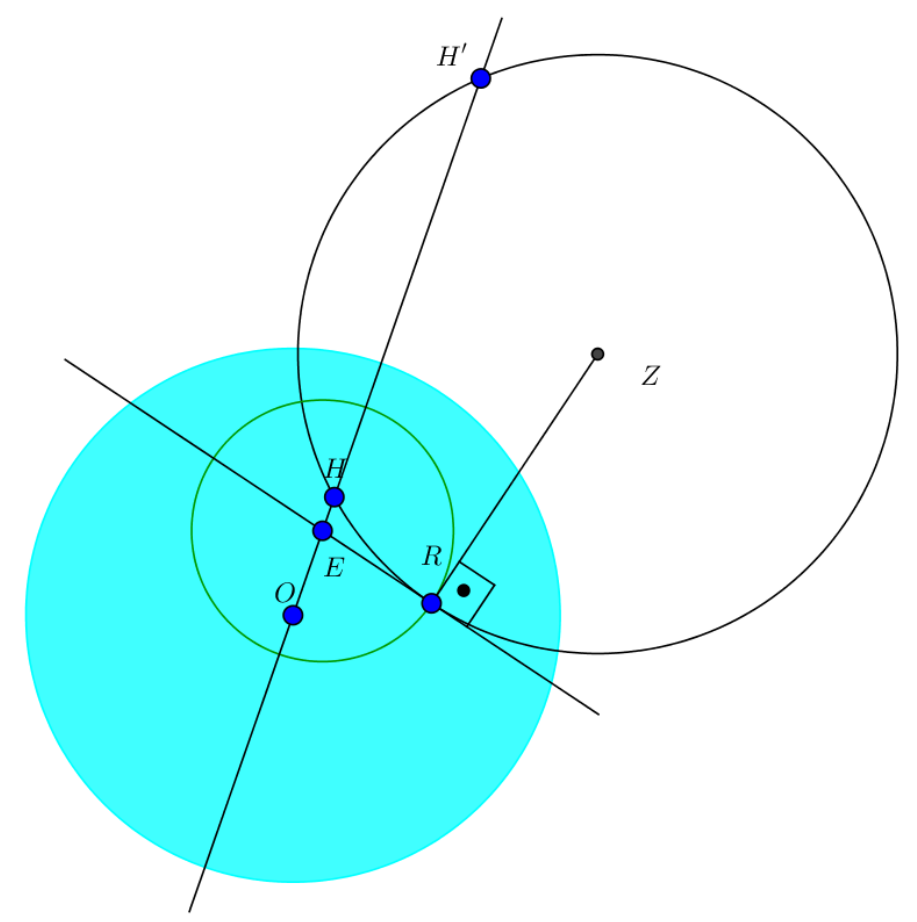

Figura 3.8: Construção do Círculo no Modelo de Poincaré

Proposição 13 O modelo do disco de Poincaré satisfaz o postulado 3 de Euclides, segundo o qual um círculo pode ser traçado com centro e raio arbitrários.

Prova. Decorre dos teoremas 12 e 13 e da construção 9.

Proposição 14 O modelo do disco do Poincaré satisfaz o postulado 4 de Euclides, segundo o qual todos os ângulos retos são iguais

Prova. Para verificar que o postulado 4 é satisfeito pelo modelo do disco de Poincaré, demonstraremos que, dados dois pares quaisquer de retas perpendiculares, no modelo do disco de Poincaré, existe uma transformação (composição de inversões circulares e rotações) que leva um par de retas no outro.

Sejam $r_{1}, s_{1}$ e $r_{2}, s_{2}$ dois pares de retas perpendiculares quaisquer, com pontos de interseção $P_{1}$ e $P_{2}$, respectivamente. Assim, existem as inversões $T_{1}$ e $T_{2}$, pelos círculos $\Gamma_{1}$ e $\Gamma_{2}$, que levam $P_{1}$ e $P_{2}$ ao centro $O$ do disco de Poincaré (proposição 12). Aplicando tais inversões aos pares de retas, observamos que 


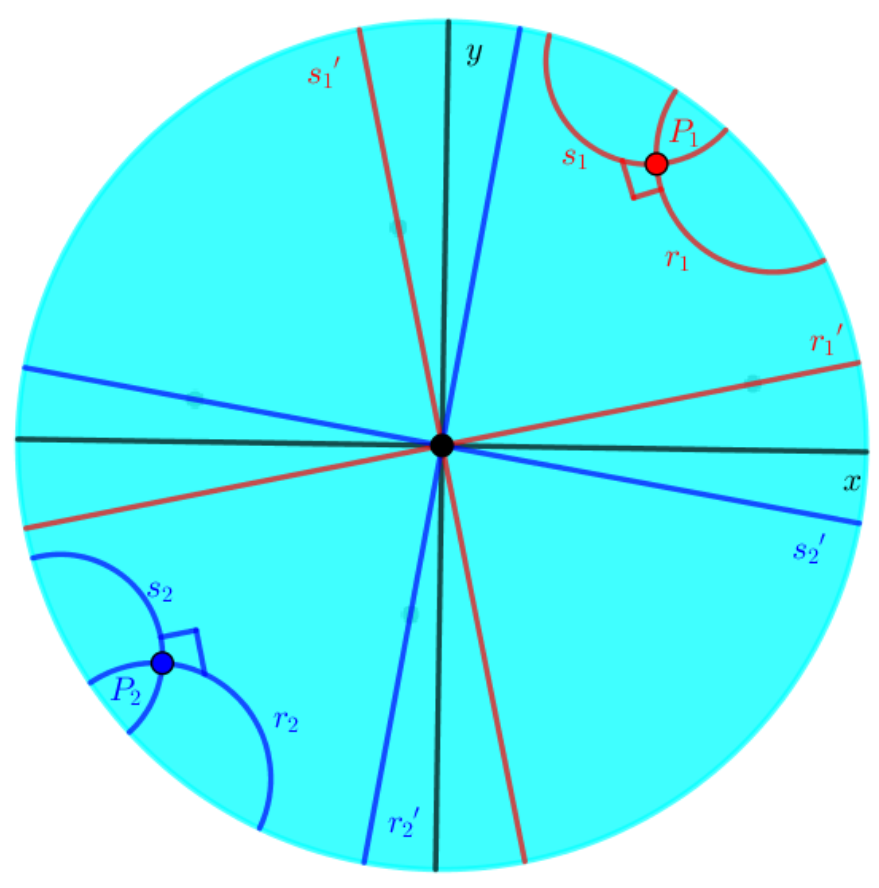

Figura 3.9: Ilustração para a prova de $\mathbf{P} 4$ no modelo do disco de Poincaré

elas serão transformadas em duas retas euclideanas, passando por $O$, ainda perpendiculares, tendo em vista que a inversão é conforme (teorema 10). Considere, agora, as rotações $R_{1}$ e $R_{2}$, que levam os pares $r_{1}^{\prime}, s_{1}^{\prime}$ e $r_{2}^{\prime}, s_{2}^{\prime}$, respectivamente, em duas retas perpendiculares, uma vertical e uma horizontal (podemos pensar nestas como o eixo $x$, horizontal e o eixo $y$, vertical, com origem $\mathrm{O}$, centro do disco de Poincaré).

Definidas a inversão e a rotação, podemos observar que, para levarmos o par retas $r_{1}, s_{1}$ em $r_{2}, s_{2}$, devemos, primeiramente, aplicar a inversão $T_{1}$ e depois a rotação $R_{1}$, que levarão $r_{1}, s_{1}$ aos eixos $x$ e $y$. Em seguida, devemos aplicar $R_{2}^{-1}$, que levará os eixos $x$ e $y$ a $r_{2}^{\prime}, s_{2}^{\prime}$ e finalmente $T_{2}^{-1}$, que levará a $r_{2}, s_{2}$. Portanto, existe uma transformação $T$, dada por $T_{2}^{-1} \circ R_{2}^{-1} \circ R_{1} \circ T_{1}$, que leva um par perpendiculares no outro.

Assim, conclui-se que, no modelo do disco de Poincaré, todos os ângulos retos são congruentes.

Após o que foi desenvolvido nesta seção, é possível perceber que o grande interesse do modelo do disco de Poincaré é justamente o fato de que ele mostra que a consistência da Geometria Hiperbólica só depende da consistência da Geometria Euclideana. 


\subsection{2}

\section{Métrica Hiperbólica}

Com relação à presente seção, devemos lembrar, primeiramente, que os aspectos desenvolvidos no tópico 2.2 permitem estabelecer um modelo analítico para o modelo do disco de Poincaré: basta considerar a absoluta como um círculo unitário no plano cartesiano $O x y$, centrado na origem.

A distância hiperbólica entre dois pontos, no modelo do disco de Poincaré, foi definida como

$$
d_{h}(A, B)=k \cdot\left|\ln \left[\left(A B, \Omega_{1} \Omega_{2}\right)\right]\right|
$$

em que $\left(A B, \Omega_{1} \Omega_{2}\right)$ é a razão cruzada entre os pontos $A, B, \Omega_{1}$ e $\Omega_{2}$, que envolve as distâncias euclideanas $d\left(\Omega_{1}, A\right), d\left(\Omega_{2}, A\right), d\left(\Omega_{1}, B\right)$ e $d\left(\Omega_{2}, B\right)$ (figura 3.4), e onde $k>0$ é uma constante arbitrária, denominada constante de Gauss.

Um fato interessante sobre tal definição de distância é que, quanto mais próximos da absoluta os pontos $A$ e $B$ estiverem, maior será a distância $d_{h}(A, B)$ e quanto mais distantes tais pontos estiverem da absoluta, menor será a distância $d_{h}(A, B)$.

Deste modo, dois pontos podem estar bem distantes do ponto de vista hiperbólico, ainda que, do ponto de vista euclideano, o comprimento do arco que os une seja pequeno. A figura 3.10 apresenta um exemplo do afirmado,com $k=1$ e raio da absoluta de medida 1 .

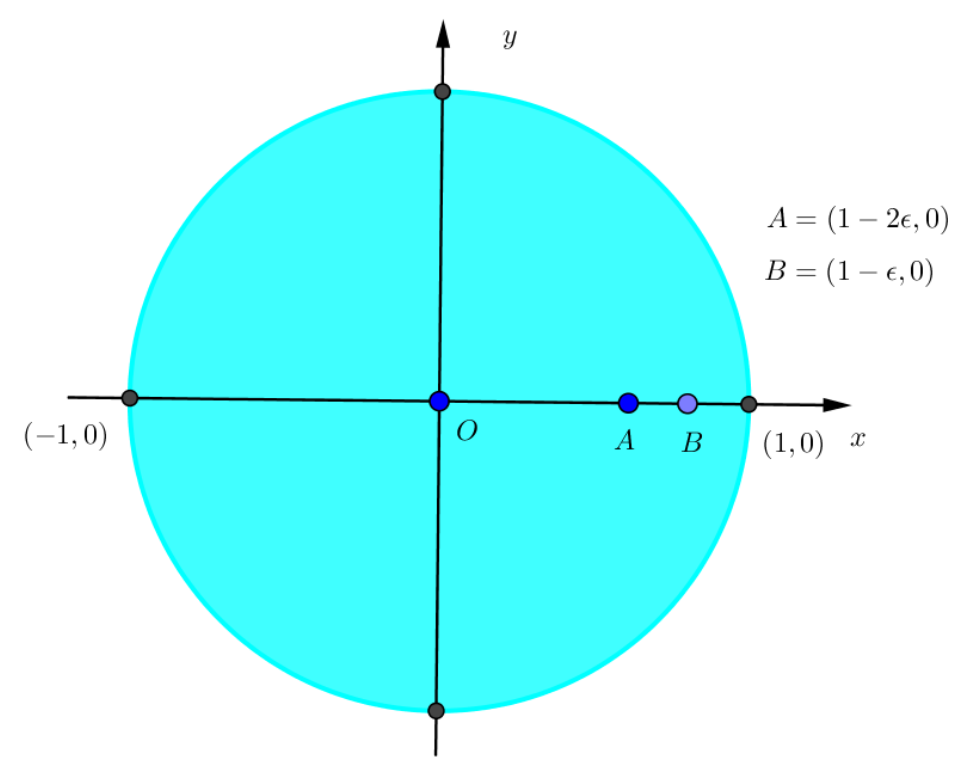

Figura 3.10: Exemplo — distância no modelo do disco de Poincaré

Na aludida figura, é possível observar que os pontos $A$ e $B$ têm coordenadas $(1-2 \epsilon, 0)$ e $(1-\epsilon, 0)$, em que $\epsilon$ tende a 0 . Estão marcados, ainda, os 
pontos ideais $\Omega_{1}=(-1,0)$ e $\Omega_{2}=(1,0)$. Assim, tem-se que $d\left(\Omega_{1}, A\right)=2-2 \epsilon$, $d\left(\Omega_{2}, A\right)=2 \epsilon, d\left(\Omega_{1}, B\right)=2-\epsilon$ e $d\left(\Omega_{2}, B\right)=\epsilon$. Logo, a distância hiperbólica entre $A$ e $B$ é dada por

$$
d_{h}(A, B)=\left|\ln \frac{2-2 \epsilon}{2 \epsilon} \cdot \frac{\epsilon}{2-\epsilon}\right|=\ln \left(\frac{2-\epsilon}{1-\epsilon}\right)
$$

Ou seja, ainda que $A$ e $B$ estejam bem próximos do ponto de vista euclideano, já que $\epsilon \rightarrow 0$, tem-se que $d_{h}(A, B)$ está próximo de $\ln 2 \approx 0,6931$.

É conveniente, ainda, definir o ângulo hiperbólico no modelo do disco de Poincaré.

Definição 23 (Ângulo Hiperbólico) O ângulo hiperbólico formado entre duas retas $r$ e s no modelo do disco de Poincaré é o ângulo formado entre as e-retas tangentes aos círculos euclideanos que contêm as retas hiperbólicas, no respectivo ponto de contato. Ou seja, o ângulo hiperbólico formado entre $r$ e s é o ângulo euclideano formado entre os círculos que contém as referidas retas (figura 3.11).

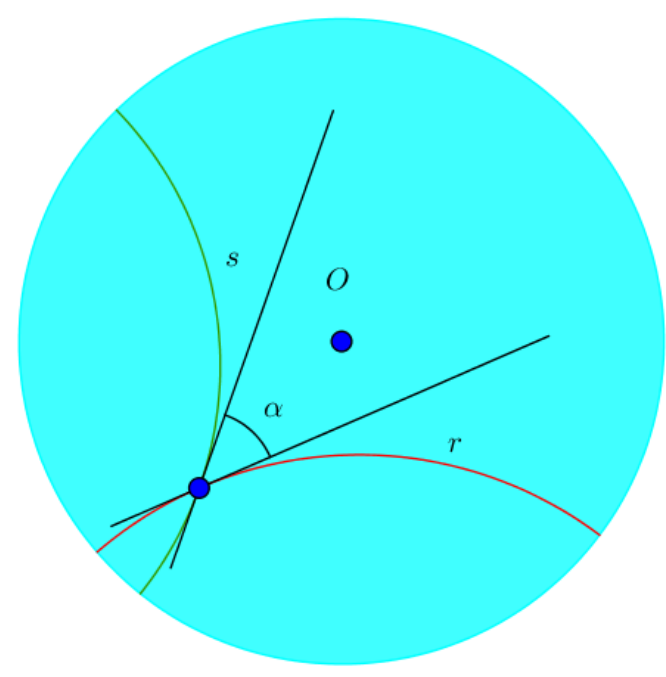

Figura 3.11: Ãngulo Hiperbólico

Deve ser apresentado, ainda, o ângulo de paralelismo, que não existe na Geometria Tradicional.

\section{Definição 24 (Ângulo de Paralelismo e Comprimento de Paralelismo)}

Dada a reta $r$ e as retas $r_{1}$ e $r_{2}$, paralelas a $r$, com ponto comum $P$, trace o segmento $\overline{A P}$, perpendicular a $r$, que passa por $P$. Dizemos que o ângulo formado entre $\overline{A P}$ e $r_{1}$, bem como entre $\overline{A P}$ e $r_{2}$ (figura 3.12) é chamado de ângulo de paralelismo. 


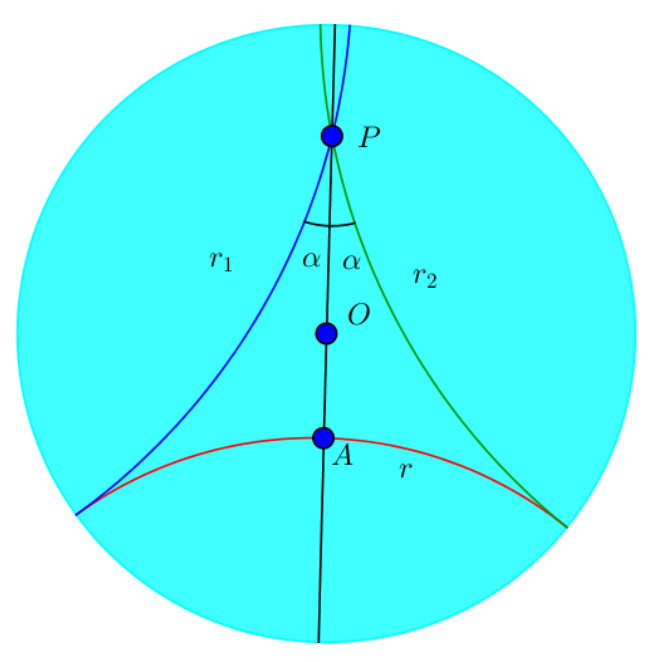

Figura 3.12: Ângulo de paralelismo

Nestas condições, dizemos que a medida hiperbólica do segmento $\overline{A P}$, perpendicular a $r$, é o comprimento de paralelismo.

Na figura 3.12, a ilustração do ângulo de paralelismo foi feita de forma conveniente com o segmento $\overline{A P}$ passando pelo ponto $O$, centro do disco de Poincaré. O interesse de a ilustração ter sido feita desta forma é o fato de que, na referida figura, há uma simetria em relação à reta $\overleftrightarrow{P A}$, o que justifica o fato de dois ângulos de paralelismo serem congruentes. Os demais casos reduzemse a este, tendo em vista que é sempre possível obter uma inversão que leve o segmento $\overline{P A}$ a passar por $O$; ademais, a inversão é conforme e preserva as distâncias hiperbólicas. Deste modo, conclui-se que dois ângulos de paralelismo são sempre congruentes.

Adiante, na seção referente à trigonometria hiperbólica, provaremos a existência de uma relação biunívoca entre a medida hiperbólica do segmento $\overline{A P}$ (comprimento de paralelismo) e a medida do ângulo de paralelismo $\alpha$.

Definido o ângulo de paralelismo, é importante destacar que, ao contrário da Geometria Euclideana, em que a unidade de medida de distâncias é arbitrária, existe uma unidade natural de comprimento, dada pela constante pela Schweikart, que é o comprimento associado ao ângulo de paralelismo medindo $\frac{\pi}{4}$. Na próxima seção, será melhor explicitado o motivo pelo qual esta pode ser considerada uma unidade natural de medida.

Definição 25 (Triângulo Hiperbólico) Um triângulo hiperbólico $A B C$ é uma reunião de segmentos finitos de retas hiperbólicas $\overline{A B} \cup \overline{A C} \cup \overline{B C}$, os quais têm apenas os vértices em comum. 


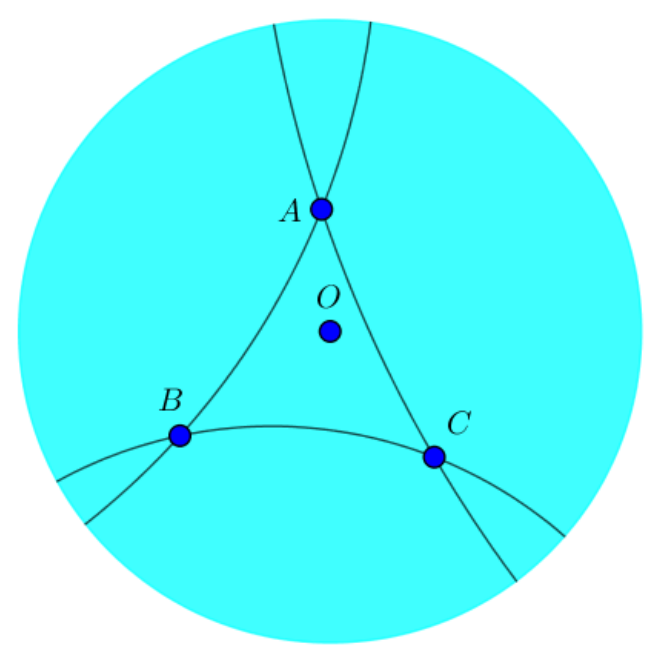

Figura 3.13: Triângulo Hiperbólico

Como é possível perceber, a definição de triângulo acima é similar à da Geometria Tradicional. Outra noção importante, no contexto da Geometria Tradicional, é a de defeito, que será exposta adiante.

Definição 26 (Defeito) Seja ABC um triângulo com ângulos internos $\alpha$, $\beta$ e $\gamma$. Nestas condições, chamamos de defeito do triângulo o número $\Delta=$ $\pi-(\alpha+\beta+\gamma)$.

É trivial que, na Geometria Tradicional, se tem $\Delta=0$, já que a soma dos ângulos internos de um triângulo euclideano é justamente $\pi$. Na Geometria Hiperbólica, $\Delta>0$, conforme será provado adiante.

Proposição 15 Todo triângulo hiperbólico tem defeito positivo.

Prova. Seja $A B C$ um triângulo hiperbólico no modelo do disco de Poincaré. Existe uma inversão, por um círculo perpendicular à absoluta, que leva o ponto $A$ no ponto $O$, centro do disco. Considere $D$ e $E$ os pontos inversos de $B$ e $C$, respectivamente, pelo mesmo círculo que leva $A$ em $O$.

Como a inversão é conforme, os ângulos do triângulo $A B C$ são os mesmos do triângulo $O D E$. Além disso, os lados $\overline{O D}$ e $\overline{O E}$ são e-retas, enquanto o lado $\overline{D E}$ continua sendo um arco de círculo.

Na figura (figura 3.14), é possível observar um triângulo euclideano com vértices em $O, D$ e $E$, que tem soma dos ângulos internos igual a $\pi$. É possível observar, ainda, o triângulo hiperbólico $O D E$, tendo sido traçadas, ainda, as tangentes ao arco de círculo de que representa o lado $D E$, nos pontos $D$ e $E$. Tendo em vista que os ângulos hiperbólicos $O \hat{D} E$ e $O \hat{E} D$ são formados entre as tangentes em $D$ e $E$ e os segmentos hiperbólicos (também e-segmentos) $\overline{O D}$ e $\overline{O E}$, respectivamente, segue que os referidos ângulos hiperbólicos têm 
medida inferior à dos ângulos euclideanos $O \hat{D} E$ e $O \hat{E} D$. Daí, segue que a soma dos ângulos do triângulo $O D E$ é inferior a $\pi$, donde segue que o defeito de tal triângulo é positivo.

Como o triângulo $O D E$ tem os mesmos ângulos de $A B C$, segue que este último triângulo também tem defeito positivo, como queríamos demonstrar.

Considerando polígonos da mesma forma que na Geometria Euclideana, podemos definir, também, o defeito do polígono como sendo a diferença entre a soma dos ângulos internos que o polígono teria na Geometria Tradicional e a efetiva soma dos seus ângulos internos na Geometria Hiperbólica. De modo mais formal,

Definição 27 (Defeito de um polígono) Considere um polígono hiperbólico de $n$ lados, com ângulos internos $\alpha_{1}, \alpha_{2}, \ldots, a_{n}$. Dizemos que o número

$$
\pi \cdot(n-2)-\sum_{i=1}^{n} \alpha_{i}
$$

é o defeito do polígono.

No que diz respeito a polígonos hiperbólicos, há uma proposição análoga à que existe para os triângulos.

Proposição 16 Todo polígono hiperbólico tem defeito positivo.

Prova. Considere $A_{1} A_{2}, \ldots, A_{n}$ um polígono hiperbólico e $P$ um ponto no interior do polígono. Traçando os segmentos hiperbólicos $\overline{P A_{1}}, \overline{P A_{2}}, \ldots, \overline{P A_{n}}$, obtemos os triângulos $P A_{1} A_{2}, P A_{2} A_{3}, \ldots, P A_{n} A_{1}$.

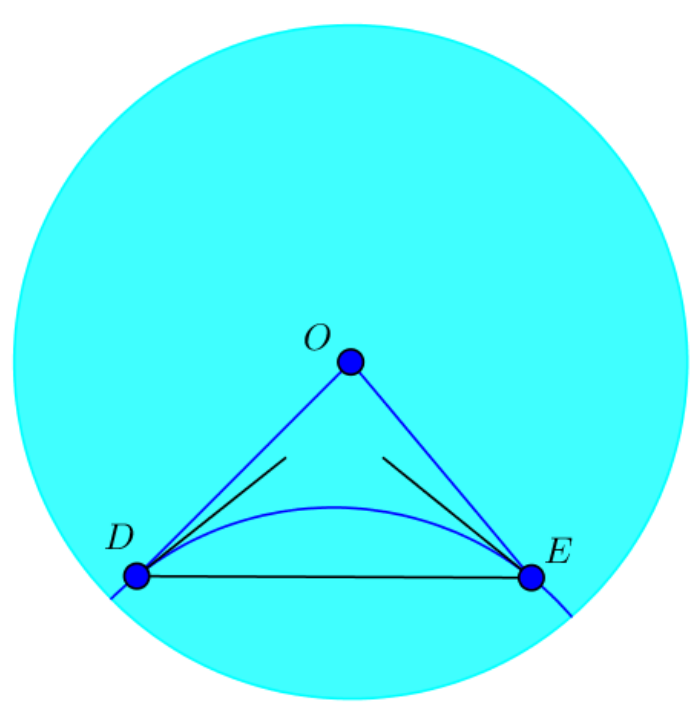

Figura 3.14: Ilustração - Defeito do Triângulo Hiperbólico 
Cada um dos triângulos acima tem três ângulos internos, um dos quais no vértice $P$. Além disso, os triângulos que têm um lado comum, digamos $\overline{P A_{j}}$, contém ângulos no vértice comum $A_{j}$, que, somados, correspondem ao ângulo interno do polígono no vértice $A_{j}$.

Deste modo, a soma dos ângulos internos, no vértice $P$, dos triângulos acima referidos é $2 \pi$ e a soma dos demais ângulos internos triângulos corresponde à soma dos ângulos internos do polígono. Assim, denotando por $\alpha_{1}, \alpha_{2}, \ldots, \alpha_{n}$ os ângulos internos do polígono, conclui-se que a soma dos defeitos de $P A_{1} A_{2}, P A_{2} A_{3}, \ldots P A_{n} A_{1}$ é dada por

$$
n \pi-\left(2 \pi+\alpha_{1}+\alpha_{2}+\ldots+\alpha_{n}\right)=\pi(n-2)-\sum_{i=1}^{n} \alpha_{i}
$$

ou seja, a soma dos defeitos dos triângulos acima referidos nada mais é do que o defeito do polígono $A_{1} A_{2} \ldots A_{n}$. Mas, o defeito de cada um dos triângulos é positivo. Daí, segue que o defeito do polígono é uma soma de quantidades positivas e, portanto, é positivo.

Após as considerações sobre o defeito de triângulos e polígonos, é possível provar um fato interessante que se dá na Geometria Hiperbólica, ao contrário do que ocorre na Geometria Tradicional. É o que se verá adiante.

Proposição $17 \mathrm{Na}$ Geometria Hiperbólica, se dois triângulos são semelhantes, então eles são congruentes.

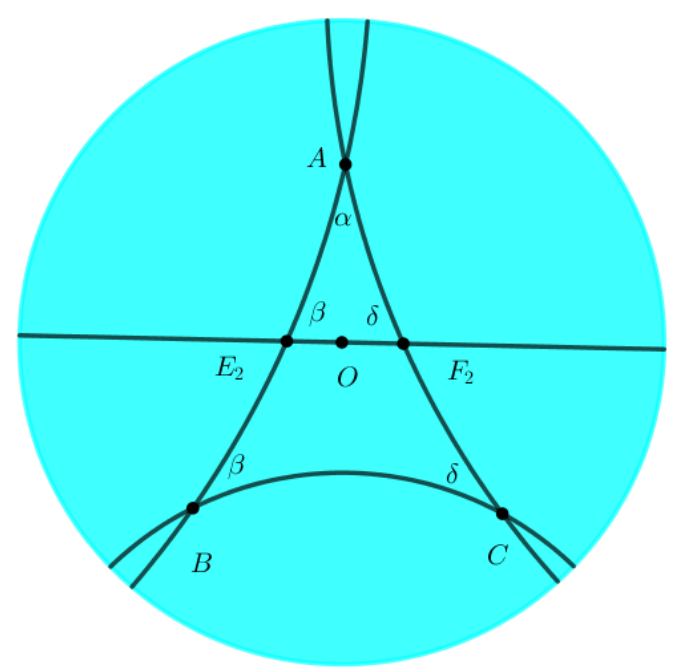

Figura 3.15: Ilustração para prova da proposição da proposição 17 (situação impossível)

Prova. Sejam $A B C$ e $D E F$ dois triângulos semelhantes, com ângulos $\hat{A}=\hat{D}=$ $\alpha, \hat{B}=\hat{E}=\beta, \hat{C}=\hat{F}=\delta$. Suponha, por absurdo, que tais triângulos não são congruentes. 
Deste modo, podemos supor, sem perda de generalidade, que $\overline{A B}>\overline{D E}$ e $\overline{A C}>\overline{D F}$. Marquemos nos lados $\overline{A B}$ e $\overline{A C}$ de $A B C$, os pontos $E_{2}$ e $F_{2}$, tais que $\overline{D E}=\overline{A E_{2}}$ e $\overline{D F}=\overline{A F_{2}}$. Assim, obtemos o triângulo $A E_{2} F_{2}$ (figura 3.15 , em que não aparece o triângulo $D E F$. A ilustração foi feita com $\overline{E_{2} F_{2}}$ passando por $O$ por questão de conveniência).

Pelo critério $L A L$ (lado-ângulo-lado), tem-se que, como $\hat{A}=\hat{D}=\alpha, \overline{D E}=$ $\overline{A E_{2}}$ e $\overline{D F}=\overline{A F_{2}}$, conclui-se que os triângulos $D E F$ e $A E_{2} F_{2}$ são congruentes. Portanto, em $D E F$, se tem $\hat{E}_{2}=\beta$ e $\hat{F}_{2}=\delta$. Daí, segue que o quadrilátero $E_{2} F_{2} C B$ tem ângulos internos $\pi-\beta, \pi-\delta, \beta$ e $\delta$, que somam $2 \pi$. Por conseguinte, temos um quadrilátero hiperbólico com defeito nulo (absurdo). Consequentemente, só podemos concluir que, se os triângulos $A B C$ e $D E F$ são semelhantes, então eles são congruentes.

No que diz respeito à área, lembremos, primeiramente, que, na Geometria Tradicional, esta é medida da seguinte forma: fixando um quadrado com lado de medida 1, que terá área 1 , observamos que um retângulo de dimensões a e $b$ terá por área $a b$, já que $a b$ quadrados de área 1 preenchem o interior do retângulo.

Traçando uma das diagonais do retângulo, obtemos dois triângulos retângulos congruentes, com catetos $a$ e $b$, donde se conclui que a área do triângulo retângulo corresponde a metade da área do retângulo, ou seja, $\frac{a b}{2}$.

Como já sabemos a área do triângulo retângulo, podemos agora obter a área de um triângulo qualquer. Seja um triângulo qualquer em que um dos lados tem medida $b$. Tracemos a altura relativa ao lado $b$, que terá medida $h$. O triângulo original ficou dividido em dois triângulos retângulos com catetos de medidas $m$ e $h$ e $n$ e $h$, respectivamente, tais que $m+n=b$. Logo, á área do triângulo será dada por

$$
\frac{m h}{2}+\frac{n h}{2}=\frac{(m+n) h}{2}=\frac{b h}{2}
$$

Tal modo de proceder, no entanto, não é possível na Geometria Hiperbólica, pois nesta não há quadrados nem retângulos. Para justificar este fato, basta observar que o defeito de qualquer polígono é positivo, de modo que não há um quadrilátero com quatro ângulos retos. Então, será necessário definir a área de forma totalmente diferente.

Do mesmo modo que na Geometria Euclideana, para definir a área de um triângulo hiperbólico, devemos associar a tal figura um número positivo que tenha as seguintes propriedades:

(i) Invariância por congruência. Isto significa que triângulos congruentes deverão ter a mesma área pela definição dada. 
(ii) Aditividade. Dado um triângulo hiperbólico $A B C$, se traçarmos uma ceviana $\overline{A D}$ que divide o triângulo original em dois outros triângulos devemos ter Área $(A B C)=$ Área $(A B D)+$ Área $(A D C)$.

Não é difícil verificar que o defeito do triângulo hiperbólico tem as propriedades acima, donde segue naturalmente a seguinte definição:

Definição 28 (Área de um Triângulo Hiperbólico) Considere um triângulo hiperbólico $A B C$ com ângulos internos $\alpha, \beta$ e $\gamma$. Então, a área do referido triângulo é dada pelo seu defeito, ou seja,

$$
\text { Área }(A B C)=\pi-(\alpha+\beta+\gamma)
$$

Em verdade, uma fundamentação mais rigorosa da área do triângulo hiperbólico como defeito dependeria de um teorema de Geometria Diferencial (Gauss-Bonnet), que está muito além dos objetivos do presente trabalho. No Capítulo 11 da obra [5], o mencionado resultado foi provado e na p. 257 deste mesmo livro, foi verificada a consequência que a área de um triângulo hiperbólico é dada pelo seu defeito, com base no modelo da pseudoesfera.

Uma observação importante sobre a definição acima é a de que os triângulos hiperbólicos, mesmo com lados arbitrariamente grandes, têm a área limitada, pois está nunca é superior a $\pi$.

Considerando o já exposto quando da demonstração de que o defeito do polígono é positivo, podemos definir a área do polígono como sendo o seu defeito.

Definição 29 (Área de um Polígono Hiperbólico) Seja $A_{1} A_{2} \ldots A_{n}$ um polígono hiperbólico com ângulos internos $\alpha_{1}, \alpha_{2}, \ldots, \alpha_{n}$. Então, a área de tal polígono é dada pelo seu defeito, ou seja,

$$
\operatorname{Área}\left(A_{1} A_{2} \ldots A_{n}\right)=\pi(n-2)-\sum_{i=1}^{n} \alpha_{i}
$$

É relevante, para o posterior desenvolvimento do trabalho, apresentar a área de um círculo hiperbólico. Porém, a prova da fórmula da área de tal figura depende de conhecimentos que serão explicitados na próxima subseção, referente à trigonometria hiperbólica. Por este motivo, a área do círculo será enunciada e provada quando do estudo do tópico referido. 


\subsection{3}

\section{Trigonometria Hiperbólica}

A trigonometria se ocupa do estudo das relações entre os lados e os ângulos de triângulo. É sabido que, na Geometria Euclideana, dado um triângulo retângulo qualquer, são definidas as relações trigonométricas seno, cosseno e tangente, dentre outras:

$\sin \alpha=\frac{\text { cateto oposto }}{\text { hipotenusa }} ; \quad \cos \alpha=\frac{\text { cateto adjacente }}{\text { hipotenusa }} ; \quad \tan \alpha=\frac{\text { cateto oposto }}{\text { cateto adjacente }}$

As funções trigonométricas também fazem sentido na Geometria Hiperbólica, mas apenas seria possível defini-las da mesma forma que a Geometria Tradicional em modelos conformes, como o do disco de Poincaré, nos quais os ângulos são medidos da mesma forma que na Geometria Euclideana. No entanto, uma resposta mais geral para o problema diz respeito a definir as funções trigonométricas hiperbólicas de forma puramente analítica, para depois utilizá-las no contexto da Geometria.

Deste modo, são introduzidas as seguintes definições das funções seno hiperbólico ( $\sinh )$ e cosseno hiperbólico (cosh):

$$
\sinh x=\frac{e^{x}-e^{-x}}{2} ; \quad \cosh x=\frac{e^{x}+e^{-x}}{2}
$$

A tangente hiperbólica é definida de maneira análoga à da trigonometria tradicional:

$$
\tanh x=\frac{\sinh x}{\cosh x}=\frac{e^{x}-e^{-x}}{e^{x}+e^{-x}}
$$

Com relação às funções trigonométricas hiperbólicas, também existe uma relação fundamental $\left(\cosh ^{2} x-\sinh ^{2} x=1\right)$. Note-se que

$$
\begin{gathered}
\cosh ^{2} x-\sinh ^{2} x=\left(\frac{e^{x}+e^{-x}}{2}\right)^{2}-\left(\frac{e^{x}-e^{-x}}{2}\right)^{2}= \\
\frac{e^{2 x}+2+e^{-2 x}}{4}-\frac{e^{2 x}-2+e^{-2 x}}{4}=\frac{2+2}{4}=1
\end{gathered}
$$

Podem ser provadas, ainda, outras relações entre as funções trigonométricas hiperbólicas que são bem análogas às da trigonometria tradicional. Uma exposição detalhada destas relações pode ser encontrada em [11], p. 401.

Voltando agora à Geometria, podemos estabelecer relações trigonométricas nos triângulos hiperbólicos, o que será feito a seguir. Antes, será necessária, 
porém, uma proposição que estabelece um resultado que será útil posteriormente.

Proposição 18 Seja $r$ uma reta hiperbólica que passa por $O$, centro da absoluta e seja $P$ um ponto sobre $r$. Nestas condições, denotando por $t$ a distância hiperbólica $d_{h}(O, P)$ e por $x$ a medida do segmento euclideano $\overline{O P}$, tem-se que são satisfeitas as seguintes relações:

$$
\sinh t=\frac{2 x}{1-x^{2}} ; \quad \cosh t=\frac{1+x^{2}}{1-x^{2}} ; \quad \tanh t=\frac{2 x}{1+x^{2}}
$$

Prova. Para provar as fórmulas acima, estamos considerando o caso em que $k=1$ e o raio do disco de Poincaré tem medida 1 . Assim, tem-se que a distância hiperbólica $d_{h}(O, P)=t$ é dada por

$$
\ln \left(\frac{1+x}{1-x}\right)
$$

Logo,

$$
\begin{gathered}
t=\ln \left(\frac{1+x}{1-x}\right) \\
e^{t}=\frac{1+x}{1-x}
\end{gathered}
$$

Daí, segue que

$$
\begin{gathered}
\sinh t=\frac{e^{t}-e^{-t}}{2}=\frac{\frac{1+x}{1-x}-\frac{1-x}{1+x}}{2}=\frac{x^{2}+2 x+1-\left(x^{2}-2 x+1\right)}{2\left(1-x^{2}\right)}= \\
=\frac{4 x}{2\left(1-x^{2}\right)}=\frac{2 x}{1-x^{2}} \\
\cosh t=\frac{e^{t}+e^{-t}}{2}=\frac{\frac{1+x}{1-x}+\frac{1-x}{1+x}}{2}=\frac{x^{2}+2 x+1+x^{2}-2 x+1}{2\left(1-x^{2}\right)}= \\
=\frac{2\left(1+x^{2}\right)}{2\left(1-x^{2}\right)}=\frac{1+x^{2}}{1-x^{2}} \\
\tanh t=\frac{\sinh t}{\cosh t}=\frac{2 x}{\frac{1-x^{2}}{1+x^{2}}}=\frac{2 x}{1+x^{2}}
\end{gathered}
$$

Teorema 14 Seja $A B C$ um triângulo retângulo hiperbólico, retângulo em $C$, com ângulos denotados por $\hat{A}, \hat{B}$ e $\hat{C}$ e lados designados por $\overline{B C}=a, \overline{A C}=b$ 
e $\overline{A B}=c$. Então, são válidas as seguintes relações trigonométricas:

(1) $\sin \hat{A}=\frac{\sinh a}{\sinh c} ; \quad \cos \hat{A}=\frac{\tanh b}{\tanh c}$

(2) $\cosh c=\cosh a \cdot \cosh b=\cot \hat{A} \cot \hat{B}$

$$
\text { (3) } \cosh a=\frac{\cos \hat{A}}{\sin \hat{B}}
$$

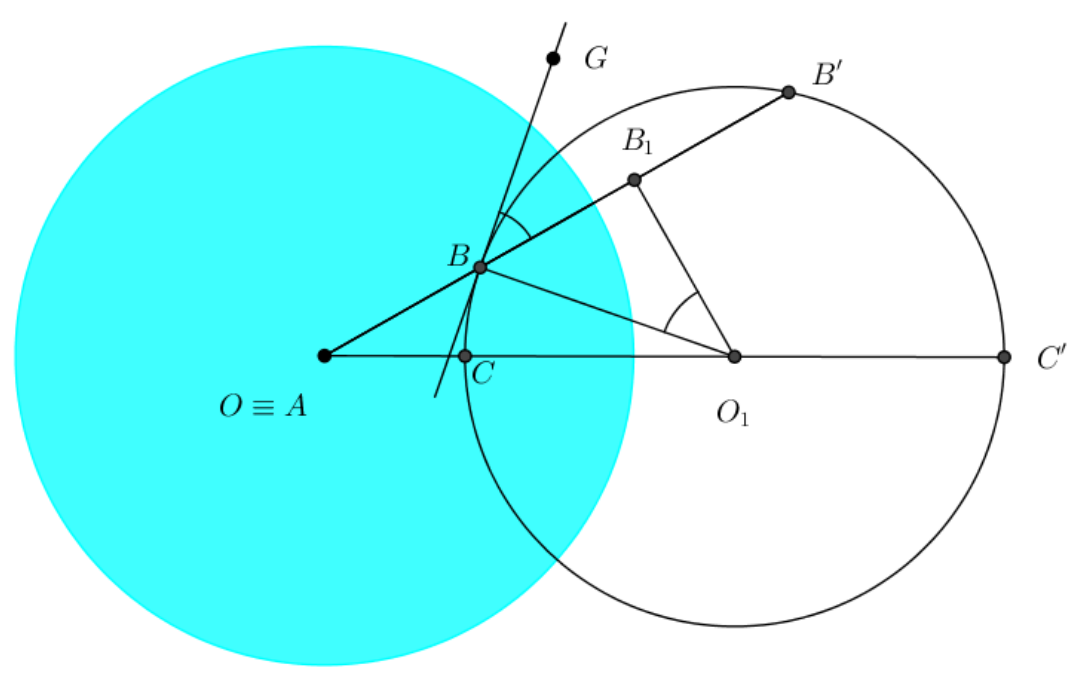

Figura 3.16: Ilustração para a prova do teorema 14

Prova. Na figura 3.16, o triângulo retângulo foi traçado com o vértice $A$ coincidente com $O$, centro da absoluta, de tal modo que dois dos seus lados $\overline{A B}=c$ e $\overline{A C}=b$ correspondem a retas euclideanas. O lado $\overline{B C}=a$ está contido numa reta hiperbólica, representada por um arco círculo perpendicular ao bordo do disco de Poincaré. Foi considerado $k=1$ e o raio da absoluta com medida 1.

Para verificar que, na figura acima o ângulo $\hat{C}$ é reto, basta observar que os pontos $O \equiv A, C$ e $O_{1}$ são colineares. Assim, como $O_{1}$ é o centro do círculo que representa a reta hiperbólica que passa por $B$ e $C, \overleftrightarrow{O_{1} C}$ é perpendicular à tangente em $C$, donde segue que, pela observação anterior, que $\overleftrightarrow{A C}$ é perpendicular à referida tangente.

O caso geral reduz-se à hipótese apresentada acima, uma vez que é sempre possível obter uma inversão que leve um dos vértices do triângulo retângulo ao centro do disco de Poincaré. Como a inversão preserva os ângulos e a razão cruzada - e, por via de consequência, a distância hiperbólica - , as operações realizadas preservarão as razões trigonométricas adiante obtidas.

Na figura acima, o segmento $\overline{O_{1} B_{1}}$ é perpendicular a $\overline{O B^{\prime}}$. Deste modo, observase que o ângulo $\hat{B}$ do triângulo $A B C$, formado pela reta tangente ao círculo 
em $B$ e a reta $\overleftrightarrow{O B}$, tem medida idêntica ao ângulo $G \hat{B} B^{\prime}$, já que são opostos pelo vértice. Daí, segue que $\hat{B}=B \hat{O}_{1} B_{1}$, já que se tem $G \hat{B} B^{\prime}+B_{1} \hat{B} O_{1}=\frac{\pi}{2}$, do mesmo modo que $B \hat{O}_{1} B_{1}+B_{1} \hat{B} O_{1}=\frac{\pi}{2}$.

Pelo teorema 8, enunciado e demonstrado no Capítulo 2, tem-se que, como os dois círculos da figura acima são perpendiculares, $B^{\prime}$ e $C^{\prime}$ são inversos de $B$ e $C$ em relação à absoluta, que tem raio 1 . Logo, denotando as medidas dos segmentos euclideanos $\overline{O B}$ por $x$ e $\overline{O C}$ por $y$ e considerando a proposição 18 , tem-se que

$$
\begin{aligned}
& \overline{B B^{\prime}}=\overline{O B^{\prime}}-\overline{O B}=\frac{1}{x}-x=\frac{1-x^{2}}{x}=\frac{2}{\sinh c} \\
& \overline{C C^{\prime}}=\overline{O C^{\prime}}-\overline{O C}=\frac{1}{y}-y=\frac{1-y^{2}}{y}=\frac{2}{\sinh b}
\end{aligned}
$$

Da figura 3.16, verifica-se, ainda, que $B_{1}$ é ponto médio de $\overline{B B^{\prime}}$, já que o triângulo $B O_{1} B$ é isósceles ( $\overline{O_{1} B}$ e $\overline{O_{1} B^{\prime}}$ são raios do mesmo círculo) e $\overline{O_{1} B_{1}}$ é altura relativa à base $\overline{B B^{\prime}}$. Além disso, $\overline{C C^{\prime}}$ é diâmetro do círculo que contém a reta hiperbólica que passa por $B$ e $C$ e, assim, $\overline{C C^{\prime}}=2 \overline{O_{1} B}$. Logo,

$$
\sin \hat{B}=\frac{\overline{B B_{1}}}{\overline{O_{1} B}}=\frac{\overline{B B^{\prime}}}{\overline{C C^{\prime}}}=\frac{\frac{2}{\sinh c}}{\frac{2}{\sinh b}}=\frac{\sinh b}{\sinh c}
$$

Uma vez que o ângulo agudo $B$ foi tomado arbitrariamente, também é válido que $\sin \hat{A}=\frac{\sinh a}{\sinh c}$. Assim, a primeira fórmula de (1) está provada.

A demonstração para a fórmula de $\cos \hat{A}$ é feita de modo análogo. Do triângulo $O O_{1} B_{1}$, retângulo em $B_{1}$, tem-se que $\cos \hat{A}=\frac{\overline{O B_{1}}}{\overline{O O_{1}}}$. Mas,

$$
\begin{aligned}
\overline{O B_{1}} & =\overline{O B}+\overline{B B_{1}}=\overline{O B}+\frac{1}{2} \cdot \overline{B B^{\prime}}=\overline{O B}+\frac{1}{2} \cdot\left(\overline{O B^{\prime}}-\overline{O B}\right)= \\
& =x+\frac{1}{2} \cdot\left(\frac{1}{x}-x\right)=x+\frac{1-x^{2}}{2 x}=\frac{1+x^{2}}{2 x}=\frac{1}{\tanh c} \\
\overline{O O_{1}} & =\overline{O C}+\overline{C O_{1}}=\overline{O C}+\frac{1}{2} \cdot \overline{C C^{\prime}}=\overline{O C}+\frac{1}{2} \cdot\left(\overline{O C^{\prime}}-\overline{O C}\right)= \\
& =y+\frac{1}{2} \cdot\left(\frac{1}{y}-y\right)=y+\frac{1-y^{2}}{2 y}=\frac{1+y^{2}}{2 y}=\frac{1}{\tanh b}
\end{aligned}
$$

Consequentemente, $\cos \hat{A}=\frac{\tanh b}{\tanh c}$.

A prova das demais relações depende apenas de manipulações algébricas:

$$
\sin ^{2} \hat{A}+\cos ^{2} \hat{A}=1
$$




$$
\begin{gathered}
\frac{\sinh ^{2} a}{\sinh ^{2} c}+\frac{\tanh ^{2} b}{\tanh ^{2} c}=1 \\
\sinh ^{2} a+\tanh ^{2} b \cosh ^{2} c=\sinh ^{2} c \\
1+\sinh ^{2} a+\left(\frac{\sinh ^{2} b}{\cosh ^{2} b}\right) \cdot \cosh ^{2} c=1+\sinh ^{2} c \\
\cosh ^{2} a \cosh ^{2} b+\sinh ^{2} b \cosh ^{2} c=\cosh ^{2} c \cosh ^{2} b \\
\cosh ^{2} a \cosh ^{2} b=\cosh ^{2} c\left(\cosh ^{2} b-\sinh ^{2} b\right) \\
\cosh ^{2} a \cosh ^{2} b=\cosh ^{2} c
\end{gathered}
$$

Como a função cosh apenas assume valores positivos, segue que $\cosh c=$ $\cosh a \cosh b$ e assim, está demonstrada a primeira igualdade em (2).

Provaremos, a seguir, a segunda igualdade em (2):

$$
\begin{aligned}
& \cot \hat{A} \cot \hat{B}=\frac{\cos \hat{A}}{\sin \hat{A}} \cdot \frac{\cos \hat{B}}{\sin \hat{B}}=\frac{\frac{\tanh b}{\tanh c}}{\frac{\sinh a}{\sinh c}} \cdot \frac{\frac{\tanh a}{\tanh c}}{\frac{\sinh c}{\sinh }}=\frac{\tanh b}{\tanh c} \cdot \frac{\sinh c}{\sinh a} \cdot \frac{\tanh a}{\tanh c} \cdot \frac{\sinh c}{\sinh b}= \\
& =\frac{\tanh a \tanh b \cosh ^{2} c}{\sinh a \sinh b}=\frac{\tanh a \tanh b \cosh a \cosh b \cosh c}{\sinh a \sinh b}=\cosh c
\end{aligned}
$$

Finalmente, provaremos a relação em (3):

$$
\begin{aligned}
& \frac{\cos \hat{A}}{\sin \hat{B}}=\frac{\frac{\tanh b}{\tanh c}}{\frac{\sinh b}{\sinh c}}=\frac{\tanh b}{\tanh c} \cdot \frac{\sinh c}{\sinh b}=\frac{\frac{\sinh b}{\cosh b}}{\frac{\sinh c}{\cosh c}} \cdot \frac{\sinh c}{\sinh b}= \\
& =\frac{\frac{1}{\cosh b}}{\frac{1}{\cosh c}}=\frac{\cosh c}{\cosh b}=\cosh a
\end{aligned}
$$

Adiante, serão enunciadas e provadas as relações trigonométricas num triângulo hiperbólico qualquer (lei dos senos e lei dos cossenos), mas será necessário utilizar, nas demonstrações, as fórmulas do seno hiperbólico da soma e do cosseno hiperbólico da soma. Por este motivo, introduziremos as fórmulas mencionadas.

Proposição 19 São válidas as seguintes relações:

(1) $\sinh (x+y)=\sinh x \cosh y+\sinh y \cosh x$;

(2) $\cosh (x+y)=\cosh x \cosh y+\sinh x \sinh y$. 
Prova. Provaremos, primeiramente, a fórmula em (1).

$$
\begin{gathered}
\sinh x \cosh y+\sinh y \cosh x= \\
=\left(\frac{e^{x}-e^{-x}}{2}\right) \cdot\left(\frac{e^{y}+e^{-y}}{2}\right)+\left(\frac{e^{y}-e^{-y}}{2}\right) \cdot\left(\frac{e^{x}+e^{-x}}{2}\right)= \\
=\frac{e^{x+y}+e^{x-y}-e^{y-x}-e^{-(x+y)}+e^{x+y}+e^{y-x}-e^{x-y}-e^{-(x+y)}}{4}= \\
=\frac{2\left(e^{x+y}-e^{-(x+y)}\right)}{4}=\frac{\left(e^{x+y}-e^{-(x+y)}\right)}{2}=\sinh (x+y)
\end{gathered}
$$

Faremos, a seguir, a demonstração da fórmula em (2).

$$
\begin{gathered}
\cosh x \cosh y+\sinh x \sinh y= \\
=\left(\frac{e^{x}+e^{-x}}{2}\right) \cdot\left(\frac{e^{y}+e^{-y}}{2}\right)+\left(\frac{e^{x}-e^{-x}}{2}\right) \cdot\left(\frac{e^{y}-e^{-y}}{2}\right)= \\
=\frac{e^{x+y}+e^{x-y}+e^{y-x}+e^{-(x+y)}+e^{x+y}-e^{y-x}-e^{x-y}+e^{-(x+y)}}{4}= \\
=\frac{2\left(e^{x+y}+e^{-(x+y)}\right)}{4}=\frac{\left(e^{x+y}+e^{-(x+y)}\right)}{2}=\cosh (x+y)
\end{gathered}
$$

Teorema 15 (Lei dos Senos e Lei dos Cossenos) Seja ABC um triângulo qualquer, no plano hiperbólico, com ângulos internos $\hat{A}, \hat{B}$ e $\hat{C}$ e lados $\overline{B C}=a, \overline{A C}=b$ e $\overline{A B}=c$. Então, são válidas as seguintes relações:

$$
\text { (1) } \frac{\sin \hat{A}}{\sinh a}=\frac{\sin \hat{B}}{\sinh b}=\frac{\sin \hat{C}}{\sinh c}
$$

(2) $\cosh c=\cosh a \cosh b-\sinh a \sinh b \cos \hat{C}$

$$
\text { (3) } \cosh c=\frac{\cos \hat{A} \cos \hat{B}+\cos \hat{C}}{\sin \hat{A} \sin \hat{B}}
$$

Prova. Primeiramente, cabe fazer uma observação: o triângulo $A B C$ (figura 3.17) foi desenhado considerando o vértice $C$ coincidente com o centro da absoluta, apenas para facilitar a visualização. A demonstração realizada a seguir será válida para um triângulo com vértices quaisquer no interior do disco de Poincaré.

Na figura abaixo, o segmento $\overline{O H}=d$ é perpendicular a $\overline{A B}$. Assim, considerando as medidas dos lados designadas no enunciado e os segmentos marcados 


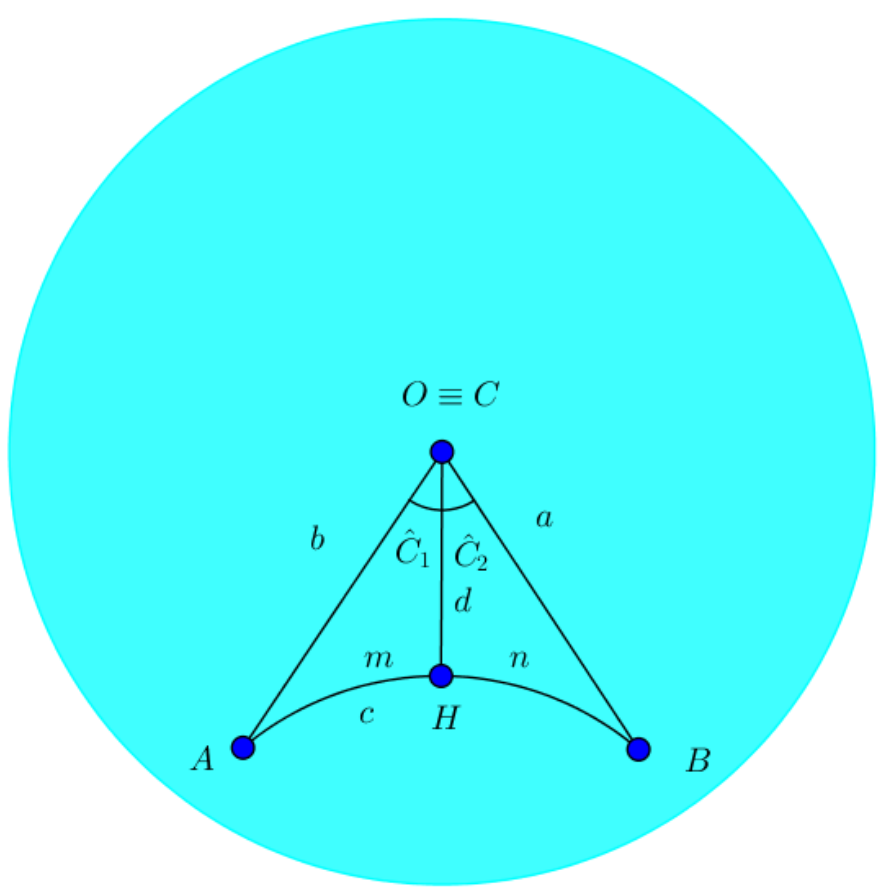

Figura 3.17: Triângulo Hiperbólico

na figura e levando em conta as fórmulas do teorema 14, tem-se que

$$
\frac{\sin \hat{A}}{\sinh a}=\frac{\sinh d}{\sinh a \sinh b} ; \quad \frac{\sin \hat{B}}{\sinh b}=\frac{\sinh d}{\sinh a \sinh b}
$$

Portanto, já está satisfeita primeira igualdade em (1). Para provar a segunda igualdade, obteremos as funções trigonométricas de $\hat{C}_{1}$ e $\hat{C}_{2}$ e aplicaremos a fórmula do seno da soma:

$$
\begin{gathered}
\sin \hat{C}_{1}=\frac{\sinh m}{\sinh b} ; \quad \cos \hat{C}_{1}=\frac{\tanh d}{\tanh b} \\
\sin \hat{C}_{2}=\frac{\sinh n}{\sinh a} ; \quad \cos \hat{C}_{2}=\frac{\tanh d}{\tanh a} \\
\sin \hat{C}=\sin \left(\hat{C}_{1}+\hat{C}_{2}\right)=\sin \hat{C}_{1} \cos \hat{C}_{2}+\sin \hat{C}_{2} \cos \hat{C}_{1}= \\
=\frac{\sinh m}{\sinh b} \cdot \frac{\tanh d}{\tanh a}+\frac{\sinh n}{\sinh a} \cdot \frac{\tanh d}{\tanh b}=\tanh d \cdot\left(\frac{\sinh m \cosh a+\sinh n \cosh b}{\sinh a \sinh b}\right)
\end{gathered}
$$

Pela fórmula (2) do teorema 14, tem-se que $\cosh a=\cosh n \cosh d$ e $\cosh b=$ $\cosh m \cosh d$. Substituindo as referidas relações em $(*)$, obtemos:

$$
\begin{gathered}
\frac{\sinh d}{\cosh d} \cdot\left(\frac{\sinh m \cosh n \cosh d+\sinh n \cosh m \cosh d}{\sinh a \sinh b}\right)= \\
\quad=\frac{\sinh d(\sinh m \cosh n+\sinh n \cosh m)}{\sinh a \sinh b} \quad(* *)
\end{gathered}
$$


Pela proposição 19, tem-se que $\sinh m \cosh n+\sinh n \cosh m=\sinh (m+n)$. Substituindo, em $(* *)$ o primeiro membro de igualdade referida pelo segundo, chegamos a:

$$
\frac{\sinh d \sinh (m+n)}{\sinh a \sinh b}=\frac{\sinh d \sinh c}{\sinh a \sinh b}
$$

Assim, do mesmo modo, se tem $\frac{\sin \hat{C}}{\sinh c}=\frac{\sinh d}{\sinh a \sinh b}$ que encerra a demonstração de (1).

Para a prova de (2) utilizaremos a fórmula do cosseno da soma:

$$
\begin{aligned}
\cos \hat{C}=\cos \left(\hat{C}_{1}+\hat{C}_{2}\right)=\cos \hat{C}_{1} \cos \hat{C}_{2}-\sin \hat{C}_{1} \sin \hat{C}_{2}= \\
=\frac{\tanh ^{2} d}{\tanh a \tanh b}-\frac{\sinh m \sinh n}{\sinh a \sinh b} \\
\cos \hat{C}=\frac{\tanh ^{2} d \cosh a \cosh b-\sinh m \sinh n}{\sinh a \sinh b}
\end{aligned}
$$

$\sinh a \sinh b \cos \hat{C}=\tanh ^{2} d \cosh a \cosh b-\sinh m \sinh n$

$\sinh a \sinh b \cos \hat{C}=\frac{\sinh ^{2} d \cosh a \cosh b}{\cosh ^{2} d}-\sinh m \sinh n$ $\sinh a \sinh b \cos \hat{C}=\frac{\left(\cosh ^{2} d-1\right) \cdot \cosh a \cosh b}{\cosh ^{2} d}-\sinh m \sinh n$ $\sinh a \sinh b \cos \hat{C}=\cosh a \cosh b-\frac{\cosh a \cosh b}{\cosh ^{2} d}-\sinh m \sinh n$

Pela fórmula (2) do teorema 14, tem-se que $\cosh d=\frac{\cosh a}{\cosh n}=\frac{\cosh b}{\cosh m}$. Daí, segue que

$$
\sinh a \sinh b \cos \hat{C}=\cosh a \cosh b-\frac{\cosh a \cosh b}{\frac{\cosh a}{\cosh n} \cdot \frac{\cosh b}{\cosh m}}-\sinh m \sinh n
$$

$\sinh a \sinh b \cos \hat{C}=\cosh a \cosh b-(\cosh m \cosh n+\sinh m \sinh n)$

$$
\begin{gathered}
\sinh a \sinh b \cos \hat{C}=\cosh a \cosh b-\cosh (m+n) \\
\sinh a \sinh b \cos \hat{C}=\cosh a \cosh b-\cosh c \\
\cosh c=\cosh a \cosh b-\sinh a \sinh b \cos \hat{C}
\end{gathered}
$$

Finalmente, provaremos a relação em (3):

$\cosh c \sin \hat{A} \sin \hat{B}=\cosh a \cosh b \sin \hat{A} \sin \hat{B}-\sinh a \sinh b \sin \hat{A} \sin \hat{B} \cos \hat{C}=$

$$
=\cosh a \cosh b \cdot \frac{\sinh d}{\sinh a} \cdot \frac{\sinh d}{\sinh b}-\sinh a \sinh b \cdot \frac{\sinh d}{\sinh a} \cdot \frac{\sinh d}{\sinh b} \cdot \cos \hat{C}=
$$




$$
\begin{aligned}
& =\frac{\sinh ^{2} d}{\tanh a \tanh b}-\sinh ^{2} d \cos \hat{C}=\frac{\sinh ^{2} d}{\tanh a \tanh b}-\left(\cosh ^{2} d-1\right) \cos \hat{C}= \\
& =\frac{\sinh ^{2} d}{\tanh a \tanh b}-\cosh ^{2} d \cos \hat{C}+\cos \hat{C}= \\
& =\frac{\sinh ^{2} d}{\tanh a \tanh b}-\cosh ^{2} d\left(\frac{\tanh ^{2} d}{\tanh a \tanh b}-\frac{\sinh m \sinh n}{\sinh a \sinh b}\right)+\cos \hat{C}= \\
& =\frac{\sinh m \sinh n \cosh ^{2} d}{\sinh a \sinh b}+\cos \hat{C}=\frac{\sinh m \sinh n \cosh a \cosh b}{\sinh a \sinh b \cosh n \cosh m}+\cos \hat{C}= \\
& =\frac{\sinh m}{\frac{\cosh m}{\sinh b}} \cdot \frac{\sinh n}{\cosh n} \frac{\sinh a}{\cosh b}+\cos \hat{C}=\frac{\tanh m}{\tanh b} \cdot \frac{\tanh n}{\tanh a}+\cos \hat{C}=\cos \hat{A} \cos \hat{B}+\cos \hat{C}
\end{aligned}
$$

o que prova a fórmula em (3).

É relevante destacar que, além das relações trigonométricas hiperbólicas acima elencadas, existem relações trigonométricas entre o ângulo de paralelismo e o comprimento de paralelismo correspondente (definição 24), as quais serão apresentadas e demonstradas adiante.

Teorema 16 (Fórmula de Lobatchevski-Bolyai) Seja u uma reta hiperbólica e $v$ e $w$ retas paralelas a u passando pelo ponto A. Nestas condições, se $\alpha$ é o ângulo de paralelismo e d é o comprimento de paralelismo, então

$$
e^{-d}=\tan \frac{\alpha}{2}
$$

Prova. Para tal demonstração, devemos esclarecer, primeiramente, que o raio do disco de Poincaré terá medida 1 e será utilizado $\mathrm{k}=1$.

Dadas as retas $u, v$ e $w$ na forma do enunciado, apliquemos uma inversão que faça com que a reta inversa de $u(r)$ passe pelo centro do disco de Poincaré, de tal modo que o pé da perpendicular baixada coincida com $O$, centro do disco de Poincaré. O inverso de $A$ será $P$ e a inversa de $v$ será $s$ (figura 3.18). A inversa de $w$ não foi mostrada acima.

O e-segmento $\overline{P Q}$ é tangente ao círculo que contém $s$, do mesmo modo que $\overline{Q X}$ também é um e-segmento tangente ao mesmo círculo, donde se conclui que $\overline{P Q}=\overline{Q X}=y$ (e-segmentos de mesma medida).

Ademais, como a inversão é conforme, conclui-se que $O \hat{P} Q$ tem a mesma medida do ângulo de paralelismo $\alpha$. Como a inversão preserva a razão cruzada, segue que $d_{h}(O, P)$ corresponde à medida do segmento de paralelismo $d$.

Da figura acima, observamos que $d_{h}(O, P)=d=\ln \left(\frac{1+x}{1-x}\right)$, em que $x$ é o comprimento euclideano do segmento $\overline{O P}$. Daí segue que, 


$$
e^{d}=\frac{1+x}{1-x} \Rightarrow x=\frac{e^{d}-1}{e^{d}+1}
$$

Considerando as medidas dos segmentos euclideanos destacadas na figura e aplicando o teorema de Pitágoras ao triângulo $O P Q$, obtemos o seguinte

$$
y^{2}=x^{2}+(1-y)^{2} \Rightarrow y^{2}=x^{2}+y^{2}-2 y+1 \Rightarrow 2 y=x^{2}+1 \Rightarrow y=\frac{x^{2}+1}{2}
$$

Na figura 3.18, está traçada a bissetriz do ângulo $O \hat{P} Q=\alpha$ e está destacada a distância euclideana de $O$ ao pé da bissetriz $(m)$. Aplicando o teorema da Bissetriz Interna ao triângulo $O P Q$, obtemos:

$$
\begin{gathered}
\frac{x}{m}=\frac{y}{1-y-m} \\
y m=x-x y-x m \\
(x+y) \cdot m=x \cdot(1-y) \\
m=\frac{x \cdot(1-y)}{x+y}
\end{gathered}
$$

Substituindo (2) em (3), obtemos

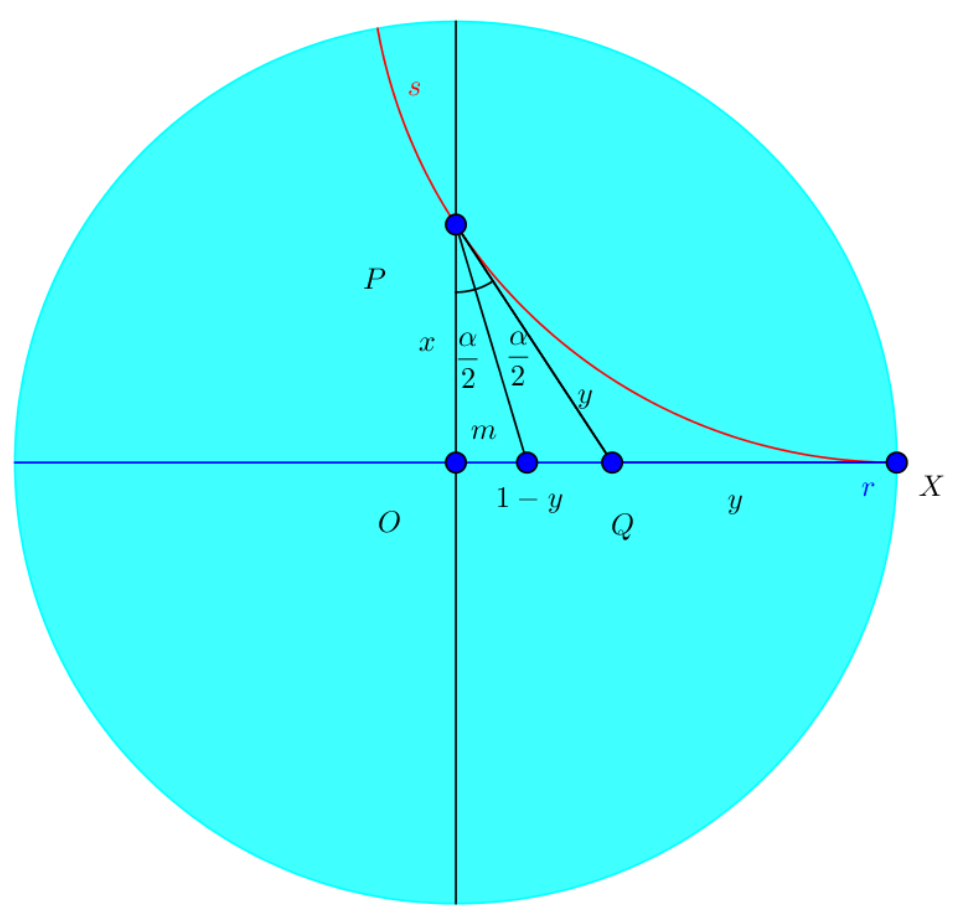

Figura 3.18: Ilustração para prova da fórmula de Lobatchevski-Bolyai 


$$
m=\frac{x \cdot\left(1-\frac{x^{2}+1}{2}\right)}{x+\frac{x^{2}+1}{2}}
$$

Fazendo as simplificações necessárias chegamos a $m=\frac{x \cdot(1-x)}{1+x}$. Daí segue que

$$
\tan \frac{\alpha}{2}=\frac{m}{x}=\frac{1-x}{1+x}
$$

Substituindo (1) em (4), obtemos

$$
\tan \frac{\alpha}{2}=\frac{1-\frac{e^{d}-1}{e^{d}+1}}{1+\frac{e^{d}-1}{e^{d}+1}}
$$

Expandindo e fazendo as simplificações necessárias, chegamos a

$$
\tan \frac{\alpha}{2}=e^{-d}
$$

como queríamos demonstrar.

A primeira observação relevante após a demonstração acima é que o ângulo de paralelismo $\alpha$ é sempre agudo, pois, com a inversão circular apresentada na figura 3.18, $\alpha$ corresponde a um ângulo de um triângulo retângulo euclideano.

É importante destacar, ainda, que, como a função exponencial é bijetora e a função tangente é bijetora no intervalo $\left(0, \frac{\pi}{2}\right)$, a fórmula de LobatchevskiBolyai permite estabelecer uma bijeção $\Pi: \mathbb{R}_{+} \backslash\{0\} \rightarrow\left(0, \frac{\pi}{2}\right)$, que associa a cada medida de segmento $t$ o seu correspondente ângulo de paralelismo $\Pi(t)$.

Um fato extremamente relevante sobre a função П é que ela é decrescente, ou seja, quanto maior o ângulo de paralelismo, menor a medida do segmento correspondente e vice-versa. Para verificar o afirmado, basta observar a função tangente é crescente no intervalo $\left(0, \frac{\pi}{2}\right)$ e a função exponencial é estritamente crescente. Daí, segue que

$$
\Pi\left(t_{1}\right)>\Pi\left(t_{2}\right) \Leftrightarrow \tan \frac{\Pi\left(t_{1}\right)}{2}>\tan \frac{\Pi\left(t_{2}\right)}{2} \Leftrightarrow e^{-t_{1}}>e^{-t_{2}} \Leftrightarrow-t_{1}>-t_{2} \Leftrightarrow t_{1}<t_{2}
$$

Além da fórmula de Lobatchevski-Bolyai, existem outras que relacionam o segmento de paralelismo e o segmento de paralelismo correspondentes, que 
serão expostas na proposição a seguir:

Teorema 17 (Outras Fórmulas) Ainda existem as seguintes fórmulas trigonométricas que relacionam o comprimento de paralelismo (t) com o ângulo correspondente $\Pi(t)$ :
(1) $\sinh t=\cot \Pi(t)$;
(2) $\cosh t=\csc \Pi(t)$;
(3) $\tanh t=\cos \Pi(t)$

Prova. Para demonstrarmos as fórmulas acima, partiremos da fórmula de Lobatchevski-Bolyai e utilizaremos identidades trigonométricas e manipulações algébricas. Sabe-se que

$$
\tan \Pi(t)=\frac{2 \tan \frac{\Pi(t)}{2}}{1-\tan ^{2} \frac{\Pi(t)}{2}}
$$

Daí, como tan $\frac{\Pi(t)}{2}=e^{-t}$, segue que

$$
\tan \Pi(t)=\frac{2 e^{-t}}{1-e^{-2 t}}
$$

Tendo em vista que $\cot \Pi(t)=\frac{1}{\tan \Pi(t)}$, chegamos a

$$
\cot \Pi(t)=\frac{1-e^{-2 t}}{2 e^{-t}}
$$

Multiplicando o numerador e o denominador do segundo membro da igualdade acima por $e^{t}$, obtemos

$$
\cot \Pi(t)=\frac{e^{t}-e^{-t}}{2}=\sinh t
$$

Assim, está provada a fórmula (1).

Para (2), utilizaremos a identidade $\cosh ^{2} t-\sinh ^{2} t=1$. Substituindo (1) na identidade mencionada, obtemos

$$
\begin{aligned}
& \cosh ^{2} t-\cot ^{2} \Pi(t)=1 \\
& 1+\cot ^{2} \Pi(t)=\cosh ^{2} t
\end{aligned}
$$

Como $1+\cot ^{2} \Pi(t)=\csc ^{2} \Pi(t)$ e tanto $\csc \Pi(t)$ quanto $\cosh t$ são positivos, conclui-se que $\csc \Pi(t)=\cosh t$. 
Por fim, concluiremos (3) com base em (1) e (2):

$$
\tanh t=\frac{\sinh t}{\cosh t}=\frac{\frac{\cos \Pi(t)}{\sin \Pi(t)}}{\frac{1}{\sin \Pi(t)}}=\cos \Pi(t)
$$

Agora, estamos em condições de justificar o motivo pelo qual a constante de Schweikart é uma constante universal do mundo hiperbólico, que fornece uma unidade natural de comprimento: é o comprimento da altura, relativa à hipotenusa, de um triângulo retângulo hiperbólico de lados infinitos.

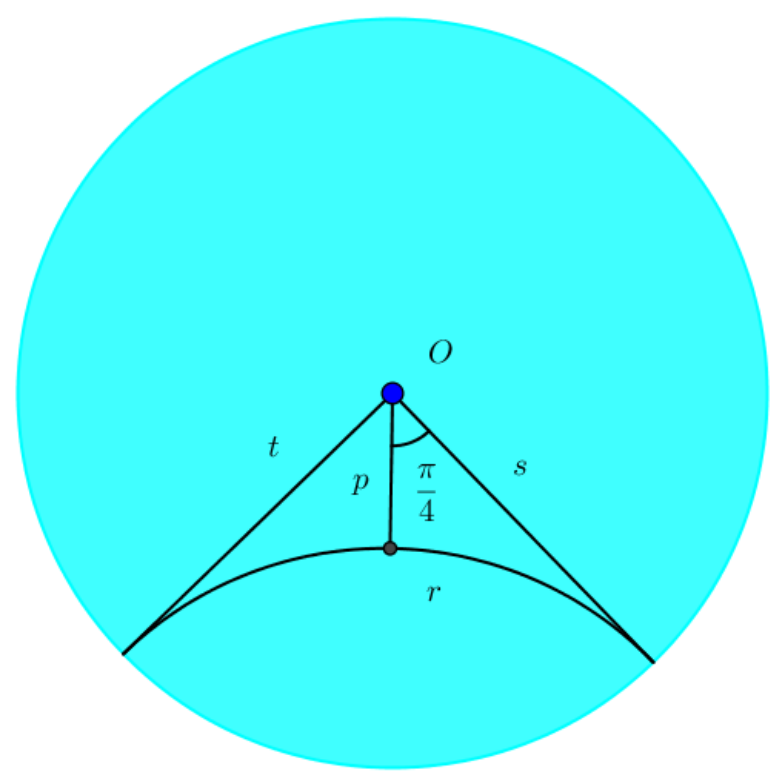

Figura 3.19: Constante de Schweikart

Na figura 3.19, tem-se que a reta hiperbólica $r$ é paralela às retas hiperbólicas $s$ e $t$ e o comprimento de paralelismo $p$ está associado ao ângulo de paralelismo $\frac{\pi}{4}$. Note que, se $k=1$ e o raio da absoluta tem medida 1 , tem-se, de uma das fórmulas do teorema 17, que

$$
\sinh p=\cot \Pi(p) \Rightarrow \sinh p=\cot \frac{\pi}{4} \Rightarrow \sinh p=1,
$$

o que contribui para simplificar muitos cálculos posteriores. Sob essa condição $p=\ln (1+\sqrt{2})$. De fato,

$$
\frac{e^{p}-e^{-p}}{2}=1 \Rightarrow\left(e^{p}\right)^{2}-2 e^{p}-1=0 \Rightarrow e^{p}=\frac{2+2 \sqrt{2}}{2} \Rightarrow p=\ln (1+\sqrt{2})
$$

Deste modo, o comprimento hiperbólico $p$ tal que $\sinh p=1$, será tomado 
como nossa unidade natural de comprimento no plano hiperbólico. Assim, ao contrário do que ocorre na Geometria Euclideana - em que as unidades de medida de segmentos são tomadas de forma arbitrária —, na Geometria Hiperbólica, existe uma unidade de comprimento que surge da própria teoria desenvolvida.

Para encerrar esta subseção, será enunciada e provada uma fórmula para a área do círculo hiperbólico. Mas, antes, temos a necessidade de provar uma fórmula para o comprimento do círculo hiperbólico e mais uma proposição auxiliar.

Proposição 20 (Comprimento do Círculo Hiperbólico) O comprimento $C$ do círculo hiperbólico é dado por $2 \pi \sinh r$.

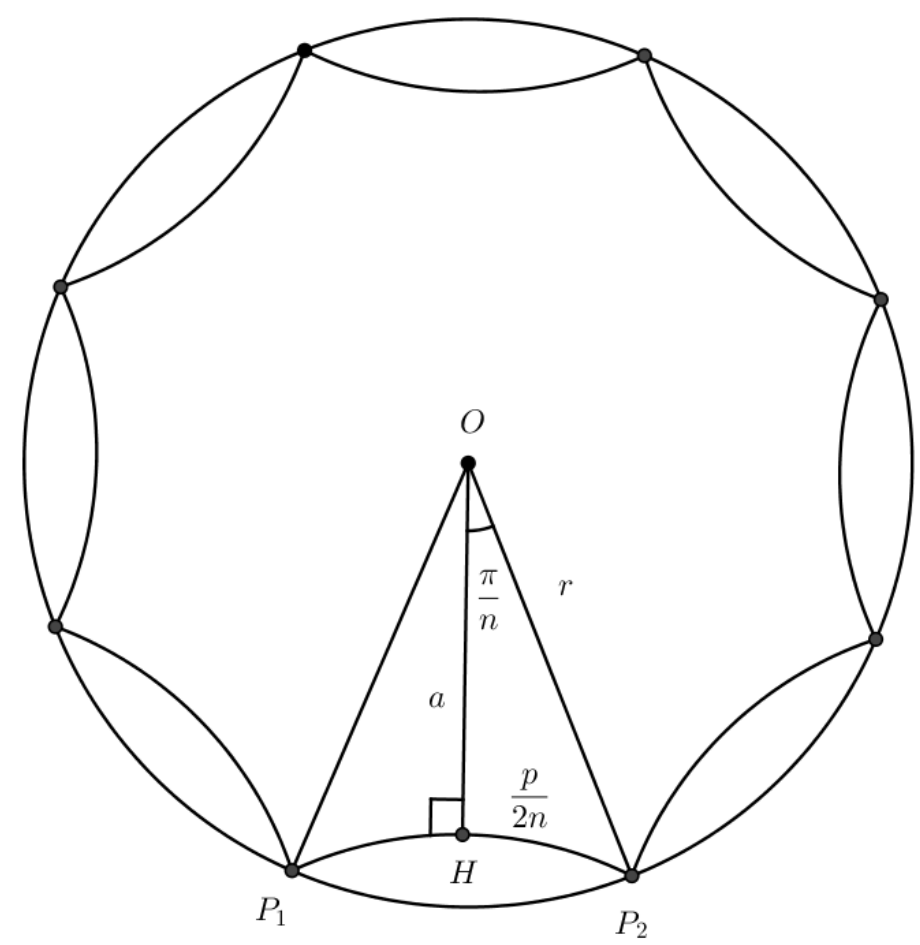

Figura 3.20: Ilustração para a prova do comprimento e da área do círculo

Prova. Na figura 3.20, está representado um polígono hiperbólico regular inscrito num círculo de raio $r$. Por questão de comodidade, o círculo foi construído com o seu centro coincidente com o centro do disco de Poincaré, mas a demonstração a ser realizada a seguir vale de modo geral (o disco de Poincaré não aparece na figura).

Para a referida prova, utilizaremos o método da exaustão. Assim, o comprimento do círculo será calculado como $C=\lim _{n \rightarrow \infty} p_{n}$, em que $p_{n}$ é o perímetro de um polígono de $n$ lados inscrito no círculo. Como o polígono é regular e 
possui $n$ lados, tem-se que cada lado tem medida $\frac{p_{n}}{n}$. Tendo em vista que o triângulo $O P_{1} P_{2}$ da figura é isósceles e $\overline{O H}$ é perpendicular a $\overline{P_{1} P_{2}}$, segue que $\overline{H P_{2}}=\frac{p_{n}}{2 n}$.

Uma vez que o ângulo central $P_{1} \hat{O} P_{2}$ tem medida $\frac{2 \pi}{n}$ e $\overline{O H}$ é bissetriz, segue que $H \hat{O} P_{2}$ tem medida $\frac{\pi}{n}$.

Denotando $p_{n}$ por $p$, por questão de simplicidade e aplicando a fórmula (1) do teorema 14, obtemos

$$
\sin \frac{\pi}{n}=\frac{\sinh \frac{p}{2 n}}{\sinh r} \Rightarrow \sinh \frac{p}{2 n}=\sin \frac{\pi}{n} \cdot \sinh r
$$

Utilizando as expansões de Taylor para $\sinh \frac{\pi}{n}$ e $\sinh \frac{p}{2 n}$, chegamos a

$$
\frac{p}{2 n}+\frac{1}{3 !}\left(\frac{p}{2 n}\right)^{3}+\frac{1}{5 !}\left(\frac{p}{2 n}\right)^{5}+\cdots=\sinh r\left[\frac{\pi}{n}-\frac{1}{3 !}\left(\frac{\pi}{n}\right)^{3}+\frac{1}{5 !}\left(\frac{\pi}{n}\right)^{5}-\ldots\right]
$$

$\frac{p}{2 n} \cdot\left[1+\frac{1}{3 !}\left(\frac{p}{2 n}\right)^{2}+\frac{1}{5 !}\left(\frac{p}{2 n}\right)^{4}+\cdots\right]=\frac{\pi}{n} \sinh r\left[1-\frac{1}{3 !}\left(\frac{\pi}{n}\right)^{2}+\frac{1}{5 !}\left(\frac{\pi}{n}\right)^{4}-\ldots\right]$

Multiplicando os dois termos da última igualdade acima por $2 n$, obtemos

$p .\left[1+\frac{1}{3 !}\left(\frac{p}{2 n}\right)^{2}+\frac{1}{5 !}\left(\frac{p}{2 n}\right)^{4}+\cdots\right]=2 \pi \sinh r\left[1-\frac{1}{3 !}\left(\frac{\pi}{n}\right)^{2}+\frac{1}{5 !}\left(\frac{\pi}{n}\right)^{4}-\ldots\right]$

Tomando, nas expressões acima, o limite quando $n$ tende a infinito, chegamos, finalmente, a $C=2 \pi \sinh r$, como queríamos demonstrar.

Enunciaremos e provaremos, na sequência, uma fórmula de suma importância para a demonstração da área do círculo.

Proposição 21 Seja ABC um triângulo retângulo hiperbólico, com ângulos internos $\hat{A}, \hat{B}$ e $\hat{C}$, este último reto. Nestas condições, denotando o defeito de $A B C$ por $K$ e considerando a notação usual para os lados do triângulo, tem-se que

$$
\tan \frac{K}{2}=\tanh \frac{a}{2} \cdot \tanh \frac{b}{2}
$$

Prova. Primeiramente, devemos observar que o defeito do triângulo retângulo $A B C$ é dado por

$$
K=\pi-\left(\frac{\pi}{2}+\hat{A}+\hat{B}\right)=\frac{\pi}{2}-(\hat{A}+\hat{B})
$$


Ademais, tem-se que, de modo geral,

$$
\tanh ^{2} \frac{x}{2}=\frac{\cosh x-1}{\cosh x+1}
$$

De fato,

$$
\begin{aligned}
\frac{\cosh x-1}{\cosh x+1}= & \frac{\frac{e^{x}+e^{-x}}{2}-1}{\frac{e^{x}+e^{-x}}{2}+1}=\frac{\frac{e^{x}-2+e^{-x}}{2}}{\frac{e^{x}+2+e^{-x}}{2}}=\frac{\frac{e^{x}-2+e^{-x}}{4}}{\frac{e^{x}+2+e^{-x}}{4}}= \\
& =\frac{\left(\frac{e^{\frac{x}{2}}-e^{-\frac{x}{2}}}{2}\right)^{2}}{\left(\frac{e^{\frac{x}{2}}-e^{-\frac{x}{2}}}{2}\right)^{2}}=\frac{\sinh ^{2} \frac{x}{2}}{\cosh ^{2} \frac{x}{2}}=\tanh ^{2} \frac{x}{2}
\end{aligned}
$$

Finalmente, provaremos a fórmula referente à proposição acima:

$$
\tanh ^{2} \frac{a}{2} \cdot \tanh ^{2} \frac{b}{2}=\frac{\cosh a-1}{\cosh a+1} \cdot \frac{\cosh b-1}{\cosh b+1}
$$

Substituindo, em (1), $\cosh a$ e $\cosh b$ por $\frac{\cos \hat{A}}{\sin \hat{B}}$ e por $\frac{\cos \hat{B}}{\sin \hat{A}}$ (teorema 14), e fazendo as simplificações necessárias, obtemos

$$
\begin{aligned}
& \tanh ^{2} \frac{a}{2} \cdot \tanh ^{2} \frac{b}{2}=\frac{(\cos \hat{A}-\sin \hat{B}) \cdot(\cos \hat{B}-\sin \hat{A})}{(\cos \hat{A}+\sin \hat{B}) \cdot(\cos \hat{B}+\sin \hat{A})}= \\
= & \frac{\left[\cos \hat{A}-\cos \left(\frac{\pi}{2}-\hat{B}\right)\right] \cdot\left[\sin \left(\frac{\pi}{2}-\hat{B}\right)-\sin \hat{A}\right]}{\left[\cos \hat{A}+\cos \left(\frac{\pi}{2}-\hat{B}\right)\right] \cdot\left[\sin \left(\frac{\pi}{2}-\hat{B}\right)+\sin \hat{A}\right]}
\end{aligned}
$$

Utilizando, em as identidades trigonométricas

$$
\begin{aligned}
& \sin x+\sin y=2 \sin \frac{x+y}{2} \cdot \cos \frac{x-y}{2} \quad \sin x-\sin y=2 \sin \frac{x-y}{2} \cdot \cos \frac{x+y}{2} \\
& \cos x+\cos y=2 \cos \frac{x+y}{2} \cdot \cos \frac{x-y}{2} \quad \cos x-\cos y=-2 \sin \frac{x+y}{2} \cdot \sin \frac{x-y}{2}
\end{aligned}
$$

chegamos a

$$
\cos \hat{A}-\cos \left(\frac{\pi}{2}-\hat{B}\right)=-2 \sin \left(\frac{\hat{A}-\hat{B}+\frac{\pi}{2}}{2}\right) \cdot \sin \left(\frac{\hat{A}+\hat{B}-\frac{\pi}{2}}{2}\right)
$$




$$
\begin{aligned}
& \sin \left(\frac{\pi}{2}-\hat{B}\right)-\sin \hat{A}=2 \sin \left(\frac{\frac{\pi}{2}-(\hat{A}+\hat{B})}{2}\right) \cdot \cos \left(\frac{\hat{A}-\hat{B}+\frac{\pi}{2}}{2}\right) \\
& \cos \hat{A}+\cos \left(\frac{\pi}{2}-\hat{B}\right)=2 \cos \left(\frac{\hat{A}-\hat{B}+\frac{\pi}{2}}{2}\right) \cdot \cos \left(\frac{\hat{A}+\hat{B}-\frac{\pi}{2}}{2}\right) \\
& \sin \left(\frac{\pi}{2}-\hat{B}\right)+\sin \hat{A}=2 \sin \left(\frac{\hat{A}-\hat{B}+\frac{\pi}{2}}{2}\right) \cdot \cos \left(\frac{\frac{\pi}{2}-(\hat{A}+\hat{B})}{2}\right)
\end{aligned}
$$

Substituindo as igualdades acima em (2) e fazendo as devidas simplificações, chegamos a

$$
\tanh ^{2} \frac{a}{2} \cdot \tanh ^{2} \frac{b}{2}=-\frac{\sin \left(\frac{\hat{A}+\hat{B}-\frac{\pi}{2}}{2}\right)}{\cos \left(\frac{\hat{A}+\hat{B}-\frac{\pi}{2}}{2}\right)} \cdot \frac{\sin \left(\frac{\frac{\pi}{2}-(\hat{A}+\hat{B})}{2}\right)}{\cos \left(\frac{\frac{\pi}{2}-(\hat{A}+\hat{B})}{2}\right)}
$$

Logo,

$$
\tanh ^{2} \frac{a}{2} \cdot \tanh ^{2} \frac{b}{2}=-\tan \left(-\frac{K}{2}\right) \cdot \tan \frac{K}{2}=\tan ^{2} \frac{K}{2}
$$

Como $\frac{K}{2}$ é um ângulo agudo, sua tangente é positiva e, assim, concluímos que $\tanh \frac{a}{2} \cdot \tanh \frac{b}{2}=\tan \frac{K}{2}$.

Finalmente, utilizando a fórmula provada acima, demonstraremos a área do círculo hiperbólico.

Teorema 18 (Área do Círculo Hiperbólico) A área de um círculo hiperbólico de raio $r$ é dada por $4 \pi \sinh ^{2} \frac{r}{2}$.

Prova. A primeira observação necessária é que a medida $a_{n}$ do apótema $\overline{O H}$ tende a $r$ quando $n$ tende a infinito, o que é visualmente claro, pois, à medida que $n$ aumenta, os lados do polígono regular se tornam cada vez menores $a_{n}$ se torna cada vez maior, se aproximando de $r$. De modo mais formal, considerando uma das relações trigonométricas do teorema 14, tem-se que

$$
\cos \frac{\pi}{n}=\frac{\tanh a_{n}}{\tanh r} \Rightarrow \tanh a_{n}=\cos \frac{\pi}{n} \cdot \tanh r
$$

Tomando, na expressão acima, o limite de n quando $n$ tende a infinito, tem-se que, como cos é contínua e tanh é um homeomorfismo, $a_{n}$, de fato, se aproxima de $r$, o que prova a afirmação. A partir de agora, o apótema será denotado simplesmente por $a$. 
Nos mesmos moldes do que foi feito para obter o comprimento do círculo, calcularemos a área do círculo a partir do limite da área $K_{n}$ do polígono regular inscrito no círculo, quando $n$ tende a infinito, ou seja $A=\lim _{n \rightarrow \infty} K_{n}$. Ou seja, utilizaremos o método da exaustão.

A área do polígono de $n$, corresponde ao seu defeito, que nada mais é do que a soma dos defeitos dos $2 n$ triângulos retângulos congruentes a $O H P_{2}$. Assim, se o defeito de $O \mathrm{HP}_{2}$ é $D$ e $K_{n}$ é a área do polígono regular, então

$$
K_{n}=2 n \cdot D \Rightarrow D=\frac{K_{n}}{2 n}
$$

Por questão de simplicidade, denotaremos, a partir de agora a área do polígono regular por $K$.

Se aplicarmos a fórmula da proposição 21 ao triângulo $O H P_{2}$, da figura 3.20, obtemos

$$
\tan \frac{D}{2}=\tan \frac{K}{4 n}=\tanh \frac{p}{4 n} \tanh \frac{a}{2}
$$

Pela expansão de Taylor de $\tan \frac{K}{4 n}$ e $\tanh \frac{p}{4 n}$, obtemos

$$
\frac{K}{4 n}+\frac{1}{3}\left(\frac{K}{4 n}\right)^{3}+\frac{2}{15}\left(\frac{K}{4 n}\right)^{5}+\cdots=\left[\frac{p}{4 n}-\frac{1}{3}\left(\frac{p}{4 n}\right)^{3}+\frac{2}{15}\left(\frac{p}{4 n}\right)^{5}-\cdots\right] \cdot \tanh \frac{a}{2}
$$

Multiplicando, por $4 n$, os dois termos da igualdade acima, obtemos

$$
K+\frac{K}{3}\left(\frac{K}{4 n}\right)^{2}+\frac{2 K}{15}\left(\frac{K}{4 n}\right)^{4}+\cdots=\left[p-\frac{p}{3}\left(\frac{p}{4 n}\right)^{2}+\frac{2 p}{15}\left(\frac{p}{4 n}\right)^{4}-\cdots\right] \cdot \tanh \frac{a}{2}
$$

Tomando $\lim _{n \rightarrow \infty}$ em ambos os lados da igualdade e lembrando que a área $A$ é dada por $\lim _{n \rightarrow \infty} K$, que $\lim _{n \rightarrow \infty} p=C=2 \pi \sinh r$ e que $a$ tende a $r$, chegamos a

$$
A=C \cdot \tanh \frac{r}{2} \Rightarrow A=2 \pi \sinh r \tanh \frac{r}{2}
$$

Para obtermos a fórmula da área do círculo, devemos observar que $\sinh r=$ $2 \sinh \frac{r}{2} \cdot \cosh \frac{r}{2}$. De fato, da primeira fórmula da proposição 19 , tem-se que

$$
\sinh r=\sinh \left(\frac{r}{2}+\frac{r}{2}\right)=\sinh \frac{r}{2} \cdot \cosh \frac{r}{2}+\sinh \frac{r}{2} \cdot \cosh \frac{r}{2}=2 \sinh \frac{r}{2} \cdot \cosh \frac{r}{2}
$$


Daí, segue que

$$
A=2 \pi \cdot\left(2 \cdot \sinh \frac{r}{2} \cdot \cosh \frac{r}{2}\right) \cdot \tanh \frac{r}{2}=4 \pi \cdot\left(\sinh \frac{r}{2} \cdot \cosh \frac{r}{2}\right) \cdot \frac{\sinh \frac{r}{2}}{\cosh \frac{r}{2}}
$$

Portanto,

$$
A=4 \pi \sinh ^{2} \frac{r}{2}
$$

como queríamos demonstrar.

\subsection{4}

\section{Construtibilidade no Plano Hiperbólico}

Vimos, na subseção 3.1.1, como são realizadas as construções da reta e do círculo no modelo do disco de Poincaré, tendo sido concluído, com base nas referidas construções, que a régua e o compasso, no modelo estudado, podem ser pensados como usos um pouco mais sofisticados dos instrumentos já conhecidos e utilizados no contexto da Geometria Tradicional. Deste modo, é possível perceber que a noção de construtibilidade com régua e compasso também faz sentido na Geometria Hiperbólica.

No entanto, como o modelo estudado é o do disco de Poincaré — que atribui novo significado a figuras já conhecidas da Geometria Euclideana -, é necessário que se tenha um certo cuidado para que não se confundam as noções de construtibilidade euclideana e hiperbólica, já que as construções realizadas no modelo anteriormente mencionada têm por base a Geometria Tradicional. Assim, denotando o disco de Poincaré por $\mathbb{D}^{2}$, é conveniente estabelecer as seguintes definições.

Definição 30 (E-construtível) Um ponto $P \in \mathbb{D}^{2}$ é dito E-construtível quando pode ser obtido, com régua e compasso, a partir dos pontos iniciais, por meio de construções euclideanas, ou seja, por meio da interseção de retas euclideanas, de retas e círculos euclideanos, ou de círculos euclideanos.

Definição 31 (H-construtível) Um ponto $P \in \mathbb{D}^{2}$ é dito H-construtível quando pode ser obtido, com régua e compasso, a partir dos pontos iniciais, por meio de construções hiperbólicas, ou seja, por meio da interseção de retas hiperbólicas, de retas e círculos hiperbólicos, ou de círculos hiperbólicos.

Alguns pontos são simultaneamente E-construtíveis e H-construtíveis, o que pode ser exemplificado pelo ponto $O$ (origem). Outros exemplos ajudarão a aclarar as definições. 


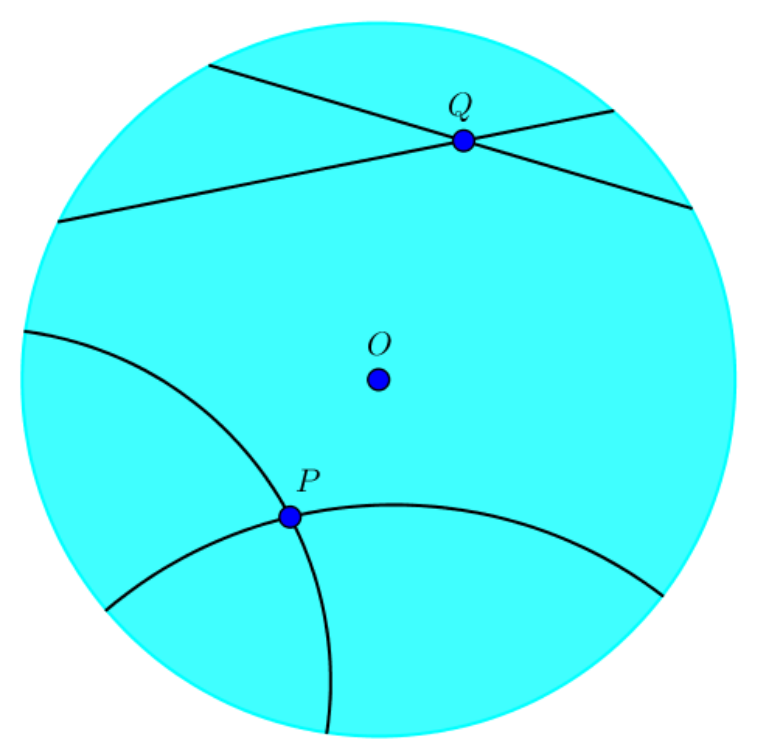

Figura 3.21: Ilustração - Ponto E-construtível e ponto $H$ construtível

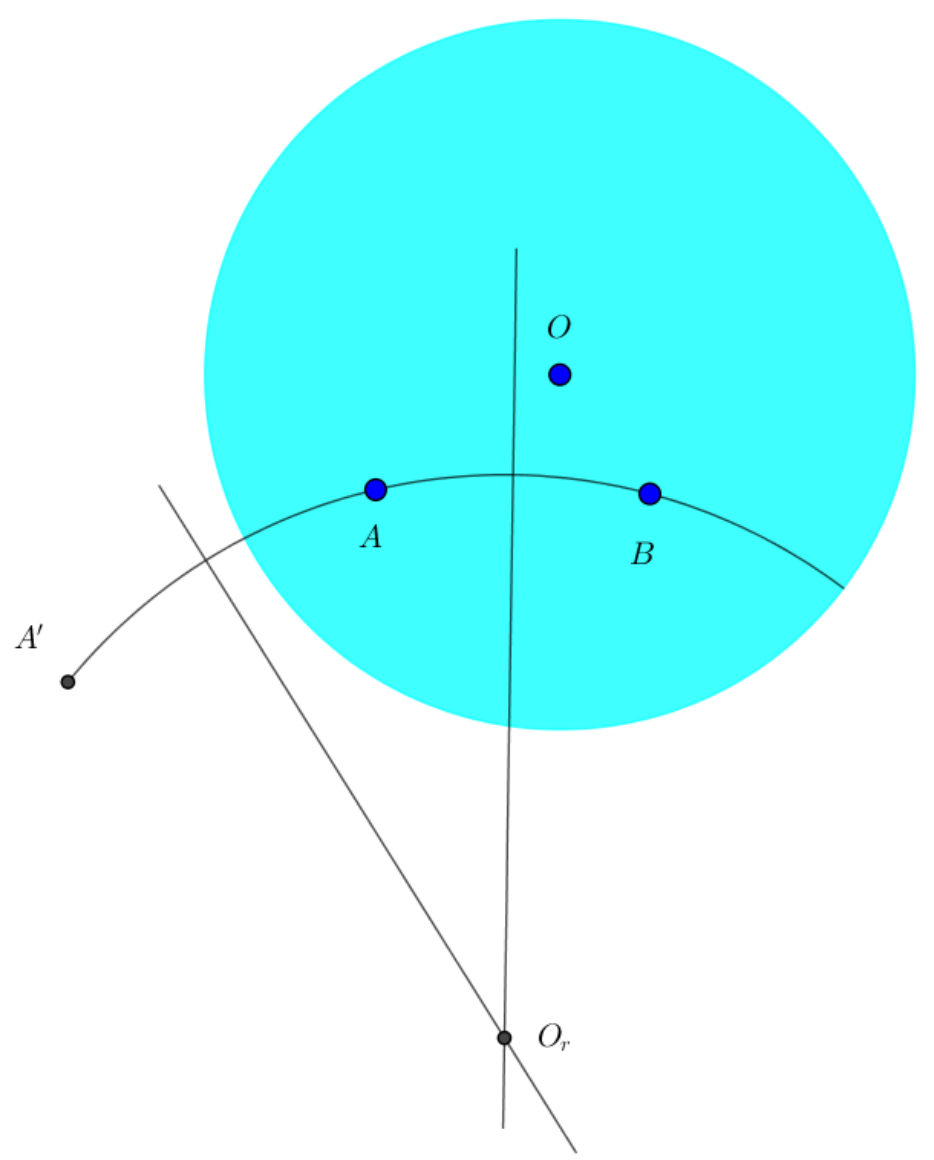

Figura 3.22: Ilustração — Reta Hiperbólica 
Na figura 3.21, o ponto $P$ é H-construtível, uma vez que é a interseção de duas retas hiperbólicas (arcos de círculos perpendiculares à absoluta). Já o ponto $Q$ é E-construtível, haja vista que é a interseção de duas retas euclideanas que não passam pelo centro do disco de Poincaré.

No segundo exemplo 3.22, relembramos a construção da reta hiperbólica que passa por dois pontos $A$ e $B$. Para realizarmos tal construção, obtemos o ponto inverso $A^{\prime}$ de $A$ e, em seguida, desenhamos as mediatrizes dos segmentos euclideanos $\overline{A A^{\prime}}$ e $\overline{A B^{\prime}}$, que se intersectarão no ponto $O_{r}$, que é o centro do círculo que contém o arco que representa a reta procurada.

O ponto $A^{\prime}$ está no exterior do disco de Poincaré, de modo que é apenas E-construtível. Do mesmo modo, as mediatrizes traçadas para obter $O_{r}$ são apenas E-construtíveis. No entanto, o arco de círculo que passa por $A$ e $B$ é H-construtível, já que representa a reta hiperbólica que passa pelos pontos mencionados.

Infere-se, do que foi estudado no Capítulo 2, seções 2.2 e 2.3, que o conjunto dos comprimentos que podem ser construídos, com régua e compasso, no plano euclideano $(E)$ é um corpo, considerando as construções anteriormente realizadas, relativas à adição, subtração, multiplicação e divisão de segmentos. Ademais, por convenção, $0 \in E$ e se considera que um numero real negativo $s \in E$ se, e somente se, $|s|$ é construtível. Observa-se, também, que o conjunto $E$ é fechado sob a operação de raiz quadrada, no seguinte sentido: se a medida $m \in E$, então $\sqrt{m} \in E$.

Outro fato importante que foi exposto nas seções supracitadas é que um ângulo $\alpha$ pode ser construído com régua e compasso no plano euclideano $\left(\mathbb{E}^{2}\right)$ se, e somente se, suas funções trigonométricas são construtíveis. Para verificar este fato, basta considerar o triângulo retângulo de hipotenusa 1 que tem um dos ângulos congruente a $\alpha$; os demais casos recaem na hipótese de $\alpha$ agudo.

Após termos relembrado os principais fatos sobre a construtibilidade no plano euclideano, introduziremos um teorema que estabelece a condição necessária e suficiente para que seja possível construir um comprimento $t$ no plano hiperbólico $\left(\mathbb{H}^{2}\right)$.

Teorema 19 (Mordukhai-Boltovskoi) Um segmento de medida $t$ é construtivel com régua e compasso no plano hiperbólico se, e somente se, $\sinh t \in E$, $\cosh t \in E$ ou $\tanh t \in E$, sendo estas condições equivalentes.

O teorema acima enunciado foi demonstrado pelos matemáticos russos Mordukhai e Boltovskoi. Uma referência para o referido teorema, em Língua Inglesa, é o livro [3] que, em seu Capítulo 11, pp. 254-275, apresenta a prova de tal resultado. Também há menção ao teorema 19 em [12], p. 482. 
Com base no teorema acima enunciado, estamos em condições de demonstrar o resultado a seguir, que diz respeito à construtibilidade de ângulos hiperbólicos.

Teorema 20 Um ângulo a pode ser construído, com régua e compasso, em $\mathbb{H}^{2}$ se, e somente se, é construtivel no plano euclideano.

Prova. Seja $\alpha$ um ângulo no plano hiperbólico. Considere um triângulo retângulo hiperbólico em que um dos ângulos internos é $\alpha$, de hipotenusa de medida correspondente ao segmento de Schweikart $(p)$, tal que $\sinh p=1$. Denotando o cateto oposto a $\alpha$ por $a$, tem-se, de uma das relações da trigonometria hiperbólica, que

$$
\sin \alpha=\frac{\sinh a}{\sinh p}=\frac{\sinh a}{1}=\sinh a
$$

Suponha que $\alpha$ possa ser construído com régua e compasso em $\mathbb{E}^{2}$. Deste modo, $\sin \alpha \in E$ e, por via de consequência $\sinh a$ é construtível no plano euclideano. Daí, segue, pelo Teorema 19, que o segmento a é construtível no plano hiperbólico. Como a hipotenusa do triângulo também é construtível, por ser a unidade, conclui-se que é possível utilizar a régua e o compasso para construir o outro cateto do triângulo retângulo, de modo que o triângulo é construtível no plano hiperbólico, bem como o ângulo $\alpha$.

Reciprocamente, suponha que $\alpha$ é construtível com régua e compasso em $\mathbb{H}^{2}$. Deste modo, é possível construir um triângulo retângulo hiperbólico com um dos ângulos internos de medida $\alpha$, hipotenusa de comprimento $p$ e cateto oposto a $\alpha$ de medida $a$. Assim, o comprimento $a$ é construtível no plano hiperbólico. Em tal triângulo, vale $\sin \alpha=\sinh a$. Como $a$ pode ser construído com régua e compasso em $\mathbb{H}^{2}$, tem-se, pelo Teorema 19, que sinh $a$ é construtível no plano euclideano e, portanto $\sin \alpha \in E$. Daí, segue que $\alpha$ é construtível no plano euclideano.

Decorre imediatamente, do teorema 20, o fato de que, em geral, não é possível realizar a trissecção de um ângulo, com régua e compasso, no contexto da Geometria Hiperbólica. Ou seja, o problema clássico da trissecção do ângulo tem uma solução trivial na Geometria Hiperbólica.

Outro corolário do teorema 20 é o fato de que um polígono regular de $n$ lados é construtível no plano hiperbólico se, e somente se, é construtível no plano euclideano. Para verificar este fato, basta observar que um polígono regular de $n$ lados tem um ângulo central associado de medida $\frac{2 \pi}{n}$.

Os teoremas apresentados nesta subseção serão fundamentais para o estudo do problema da quadratura do círculo no plano hiperbólico, que será desenvolvido no Capítulo 4. 


\section{4}

\section{Quadratura do Círculo no Plano Hiperbólico}

\section{1}

\section{Construção de um Quadrado e de um Círculo de mesma Área no Modelo do Disco de Poincaré com Régua e Compasso Euclideanos}

Na presente seção, será apresentado um exemplo de construção de um quadrado e de um círculo de mesma área no modelo do disco de Poincaré, baseada no artigo [2], publicado em 2014. A construção ocorrerá no plano euclideano, com régua e compasso euclideanos. A construtibilidade hiperbólica (i. e., intrínseca) de tais objetos decorre dos teoremas 19 e 20 da Subseção 3.1.4.

Em verdade, será realizada a construção de um caso particular, em que o centro do quadrado e do círculo coincidem com o centro $O$ do disco de Poincaré, o que não implica perda de generalidade. Para realizar tal intento, a primeira observação necessária é que será utilizada a fórmula de distância do referido modelo, com $k=1$ e com o raio da absoluta igual a 1 . Assim, o comprimento $\overline{O X}$, de um segmento que passa pelo centro do disco é dada por

$$
\ln \left(\frac{1+x}{1-x}\right)
$$

em que $x$ é a distância euclideana entre $O$ e $X$.

É relevante destacar, também, que o quadrado, na Geometria Hiperbólica, é um polígono com quatro lados congruentes e quatro ângulos agudos e de mesma medida, já que o defeito de todo polígono, em tal Geometria, é sempre positivo (proposição 16).

Pela definição 29, a área do quadrado corresponde ao seu defeito, isto é, à diferença entre a soma dos ângulos internos que tal polígono teria na Geometria Euclideana $(2 \pi)$ e a que efetivamente tem na Geometria Hiperbólica. Denotando por $\sigma$ o ângulo interno do quadrado, concluímos que a sua área é dada por $2 \pi-4 \sigma$.

Considere-se, agora, um círculo centrado em $O$, em que a outra extremidade de um dos raios é $P$, tal que a distância euclideana de $O$ a $P$ é $\frac{1}{2}$. Daí, 
segue que

$$
d_{h}(O, P)=\ln \left(\frac{1+\frac{1}{2}}{1-\frac{1}{2}}\right)=\ln \left(\frac{\frac{3}{2}}{\frac{1}{2}}\right)=\ln 3
$$

Uma vez obtida medida hiperbólica do raio do círculo (ln 3), concluímos que a sua área será dada por

$$
\begin{aligned}
4 \pi \cdot\left(\frac{e^{\frac{\ln 3}{2}}-e^{-\frac{\ln 3}{2}}}{2}\right)^{2} & =4 \pi \cdot\left(\frac{e^{\ln \sqrt{3}}-e^{\ln \frac{1}{\sqrt{3}}}}{2}\right)^{2}=4 \pi \cdot\left(\frac{\sqrt{3}-\frac{1}{\sqrt{3}}}{2}\right)^{2} \\
& =4 \pi \cdot\left(\frac{3+\frac{1}{3}-2}{4}\right)=\frac{4 \pi}{3}
\end{aligned}
$$

Como queremos construir um quadrado de mesma área $\frac{4 \pi}{3}$, devemos obter o ângulo interno $\sigma$ :

$$
2 \pi-4 \sigma=\frac{4 \pi}{3} \Rightarrow 4 \sigma=2 \pi-\frac{4 \pi}{3} \Rightarrow 4 \sigma=\frac{2 \pi}{3} \Rightarrow \sigma=\frac{\pi}{6}
$$

Note que o ângulo assim obtido $\left(\sigma=\frac{\pi}{6}\right)$ pode ser construído com régua e compasso do ponto de vista euclideano e, portanto, também é construtível no plano hiperbólico (teorema 20).

Além disso, observe que o comprimento hiperbólico do raio (ln 3$)$ é construtível em $\mathbb{H}^{2}$ pelo teorema 19, haja vista que $\sinh (\ln 3)$ é um número racional e está em $E$; o comprimento do raio também é euclideanamente construtível, pois sua medida euclideana corresponde a metade do raio euclideano da absoluta.

Vejamos como construir, com régua e compasso, o quadrilátero regular hiperbólico e o círculo de área $\frac{4 \pi}{3}$, ambos contidos num disco de Poincaré (também construído). Note que a construção dos três objetos é imbricada: eles não são construídos independentemente.

Pelos teoremas 19 e 20, da subseção 3.1.4, segue, da construtibilidade euclideana, que tal exemplo poderia ser obtido intrinsecamente no plano hiperbólico (i. e., com régua e compasso hiperbólicos), embora não tenhamos uma receita explícita.

Para realizarmos a construção, devemos proceder da seguinte forma:

1 - construir dois triângulos $A B C$ e $A D K$ congruentes e refletidos, com ângulos internos $\frac{\pi}{6}, \frac{\pi}{3}$ e $\frac{\pi}{2}$, de tal modo que o ângulo $\hat{A}$, de medida $\frac{\pi}{6}$, seja comum aos dois triângulos (figura 4.1). Para a realizar a construção dos triângulos, pode-se, primeiramente, construir um triângulo equilátero e depois 
traçar a bissetriz de um dos ângulos de medida $\frac{\pi}{3}$, ou então, considerar que os triângulos $A B C$ e $A D K$ têm um dos catetos com medida que corresponde a metade da medida da hipotenusa. Os catetos $\overline{B C}$ e $\overline{D K}$, dos triângulos $A B C$ e $A D K$, respectivamente, terão interseção no ponto $E$;

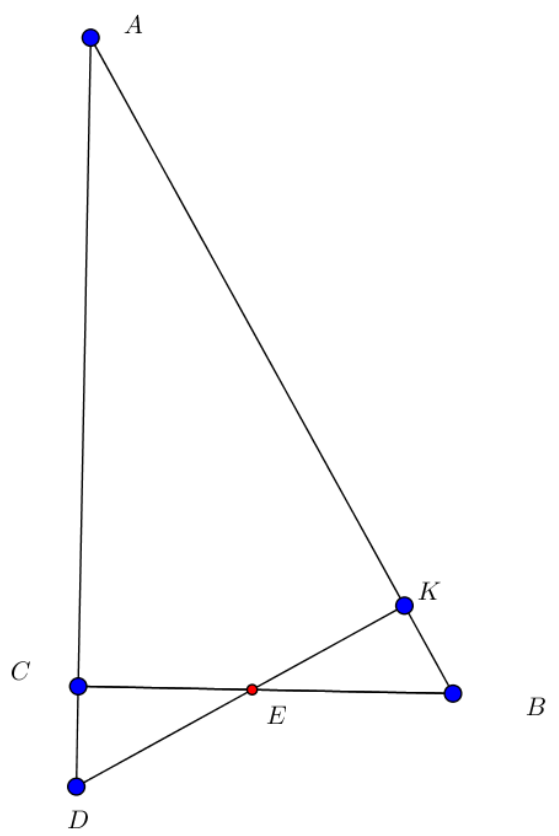

Figura 4.1: Quadrado e Círculo de Mesma Área - Parte 1

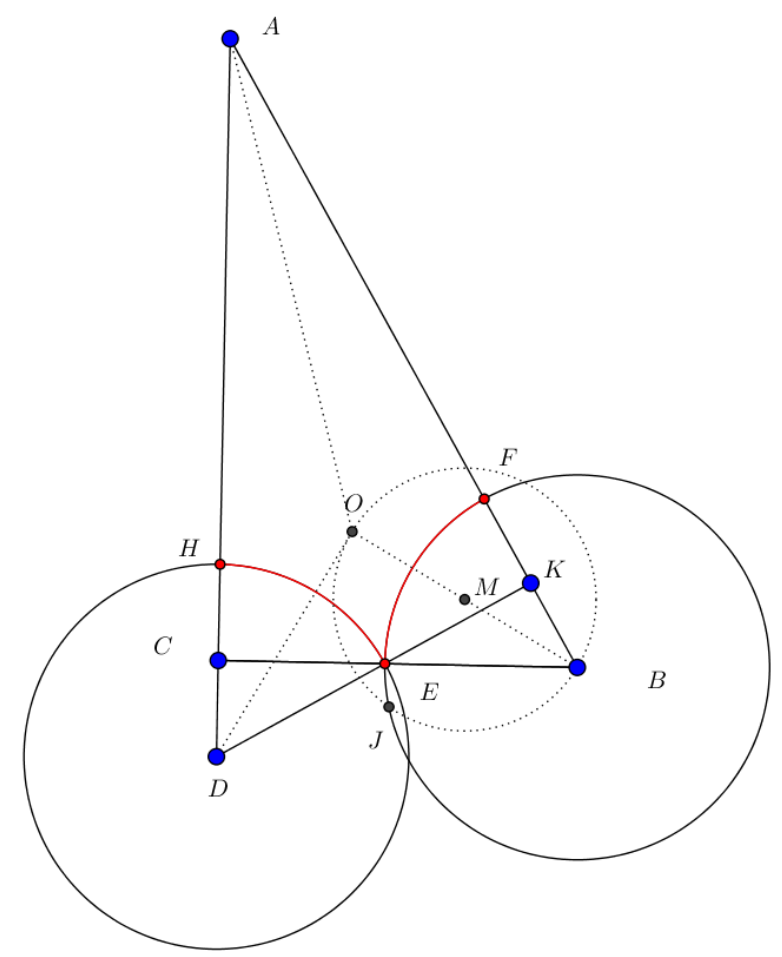

Figura 4.2: Quadrado e Círculo de Mesma Área - Parte 2 
2 - traçar os círculos de centros $B$ e $D$ e raios de medida $\overline{D E}=\overline{B E}$ (figura 4.2), que se intersectarão $\overline{A B}$ e $\overline{A D}$ nos pontos $F$ e $H$, respectivamente. Os arcos de círculos delimitados por $E$ e $F$ e por $E$ e $H$ correspondem a dois lados do quadrado que se pretende construir;

3 - obter o ponto $O$, que é o incentro comum aos dois triângulos retângulos. Para tanto, basta traçar duas das bissetrizes dos ângulos de $A B C$ e $A D K$, que se intersectarão em $O$ (figura 4.2);

4 - traçar uma circunferência, de diâmetro $\overline{O B}$, que cortará o círculo centrado em $B$ no ponto $J$. O segmento $\overline{O J}$, corresponde ao raio do disco de Poincaré (a ser construído; figura 4.2);

5 - para obter o último vértice e os outros dois lados do quadrado, devemos fazer a reflexão dos círculos dos centros $B$ e $D$, pela e-reta $\overleftrightarrow{H F}$, suporte da diagonal oposta a $E$. Estes dois últimos círculos terão dois pontos de interseção, um dos quais é o vértice $G$ do quadrado. Os arcos de círculo delimitados por $F$ e $G$ e $H$ e $G$ são os outros lados do quadrado (figura 4.3).

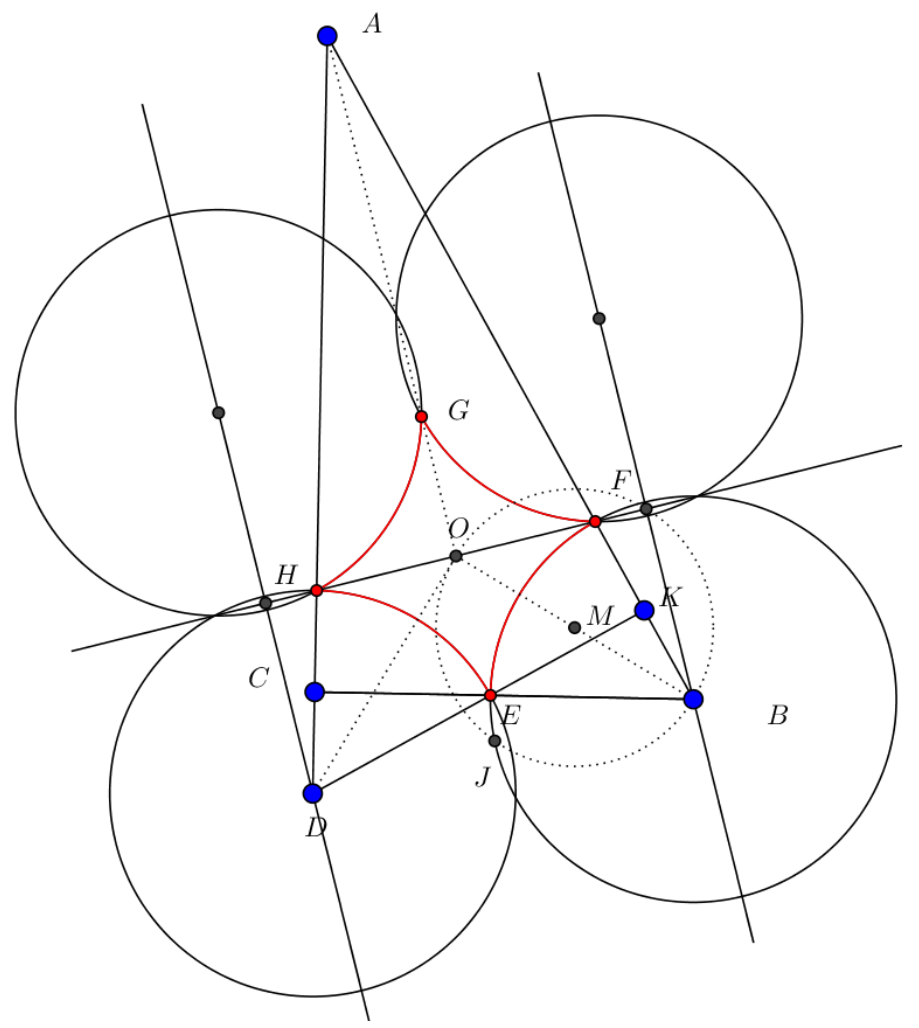

Figura 4.3: Quadrado e Círculo de Mesma Área - Parte 3

6 - para construirmos o círculo de mesma área que o quadrado $E F G H$, devemos lembrar que o raio do disco de Poincaré é $\overline{O J}$, que corresponde à medida euclideana 1 e que o círculo a ser traçado tem centro $O$ e raio euclideano $\frac{1}{2}$. Assim, para desenhar o círculo em questão, basta obter o ponto médio $N$ 
de $\overline{O J}$ e traçar o círculo procurado, que tem raio $\overline{O N}$. Após desenharmos o disco de Poincaré com raio $\overline{O J}$, a construção estará finalizada (figura 4.4).

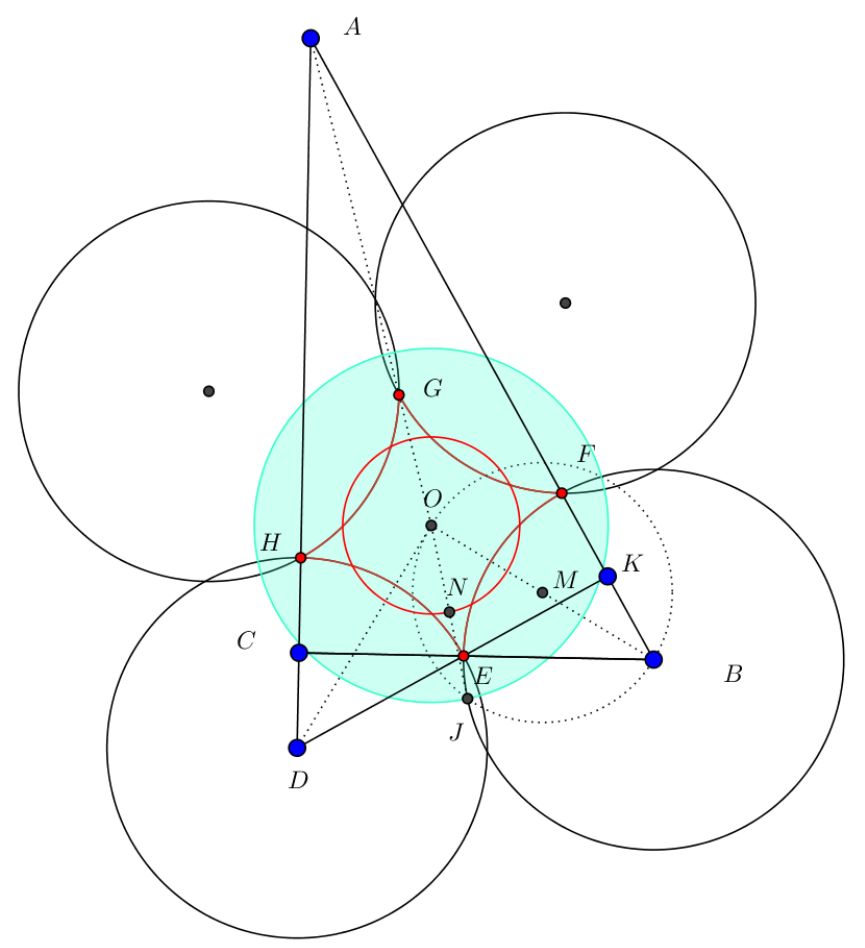

Figura 4.4: Quadrado e Círculo de Mesma Área - Construção Final

Para justificarmos a construção acima, demonstraremos as propriedades da figura em proposições separadas.

Proposição 22 Os triângulos DCE e BKE são congruentes, e, consequentemente, $\overline{B E}=\overline{D E}$.

Prova. Os triângulos $A B C$ e $A D K$ foram construídos de tal forma a serem congruentes. Assim, tem-se que $c=\overline{A B}=\overline{A D}$ (hipotenusas), $b=\overline{A C}=$ $\overline{A K}$ (catetos opostos aos ângulos $\hat{B}=\hat{D}$ ) e $a=\overline{B C}=\overline{D K}$ (catetos opostos ao ângulo $\hat{A})$. Daí, segue que

$$
\begin{aligned}
& \overline{C D}=\overline{A D}-\overline{A C}=c-b \\
& \overline{B K}=\overline{A B}-\overline{A K}=c-b
\end{aligned}
$$

Portanto, $\overline{B K}=\overline{C D}$. Ademais, $C \hat{E} D=K \hat{E} B$, pois são ângulos opostos pelo vértice, e, ainda, se tem, pela hipótese da construção, que $E \hat{D} C=E \hat{B} K$. Logo, pelo critério $L A A_{o}$ (lado-ângulo-ângulo oposto), os triângulos $D C E$ e $B K E$ são congruentes, como queríamos demonstrar e, por via de consequência, $\overline{B E}=\overline{D E}$. 
Proposição $23 \overline{A E}$ é a bissetriz interna relativa ao ângulo $\hat{A}$ dos triângulos $A B C$ e $A D K$.

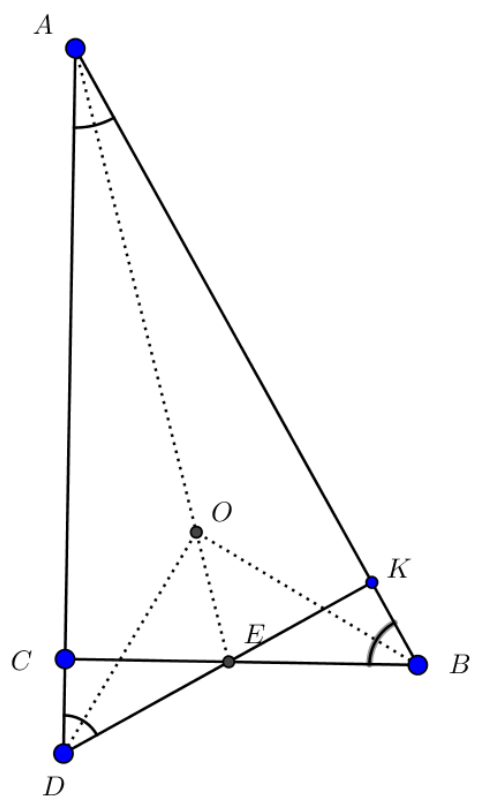

Figura 4.5: Justificativa - Construção do Quadrado e do Círculo de Mesma Área - Parte 1

Prova. Na figura 4.5, vemos que os triângulos congruentes $D C E$ e $B K E$ são semelhantes ao triângulo $B C A$, já que todos estes têm os mesmos ângulos internos de medidas $\frac{\pi}{3}, \frac{\pi}{2}$ e $\frac{\pi}{6}$.

Da semelhança entre os triângulos $D C E$ e $B C A$, tem-se que

$$
\frac{\overline{A C}}{\overline{C E}}=\frac{\overline{A B}}{\overline{D E}}
$$

Mas $\overline{D E}=\overline{B E}$, donde se conclui que

$$
\frac{\overline{A C}}{\overline{C E}}=\frac{\overline{A B}}{\overline{B E}}
$$

Considerando a última relação acima, concluímos, com base na recíproca do teorema da bissetriz interna aplicada ao triângulo $A B C$, que $\overline{A E}$ é bissetriz interna do ângulo $\hat{A}$.

Proposição $24 O$ ponto $O$, incentro do triângulo $A B C$, também é incentro do triângulo $A D K$.

Prova. Tracemos a bissetriz interna do ângulo $\hat{B}$ do triângulo $A B C$. A referida ceviana encontrará $\overline{A E}$ no ponto $O$, incentro de $A B C$. Desenhemos, também, o segmento $\overline{D O}$ (figura 4.5 ). 
Pelo critério $L A L$ (lado-ângulo-lado), tem-se que os triângulos $A O D$ e $A O B$ são congruentes, já que $\overline{A O}$ é lado comum, $B \hat{A} O=O \hat{A} D$ e $\overline{A B}=\overline{A D}$ pela hipótese da construção.

Consequentemente, $A \hat{D} O=O \hat{B} A=\frac{A \hat{B} C}{2}$. Mas, $A \hat{B} C=A \hat{D} K$, donde segue que $A \hat{D} O=\frac{A \hat{D} K}{\stackrel{2}{2}}$. Assim, $\overleftrightarrow{D O}$ é bissetriz interna do ângulo $\hat{D}$ do triângulo $A D K$. Como $\overleftrightarrow{A E}$ também é bissetriz interna de um dos ângulos do triângulo $A D K$, conclui-se que $O$ também é incentro de $A D K$.

Proposição 25 A reta $\overleftrightarrow{H F}$ passa pelo ponto $O$

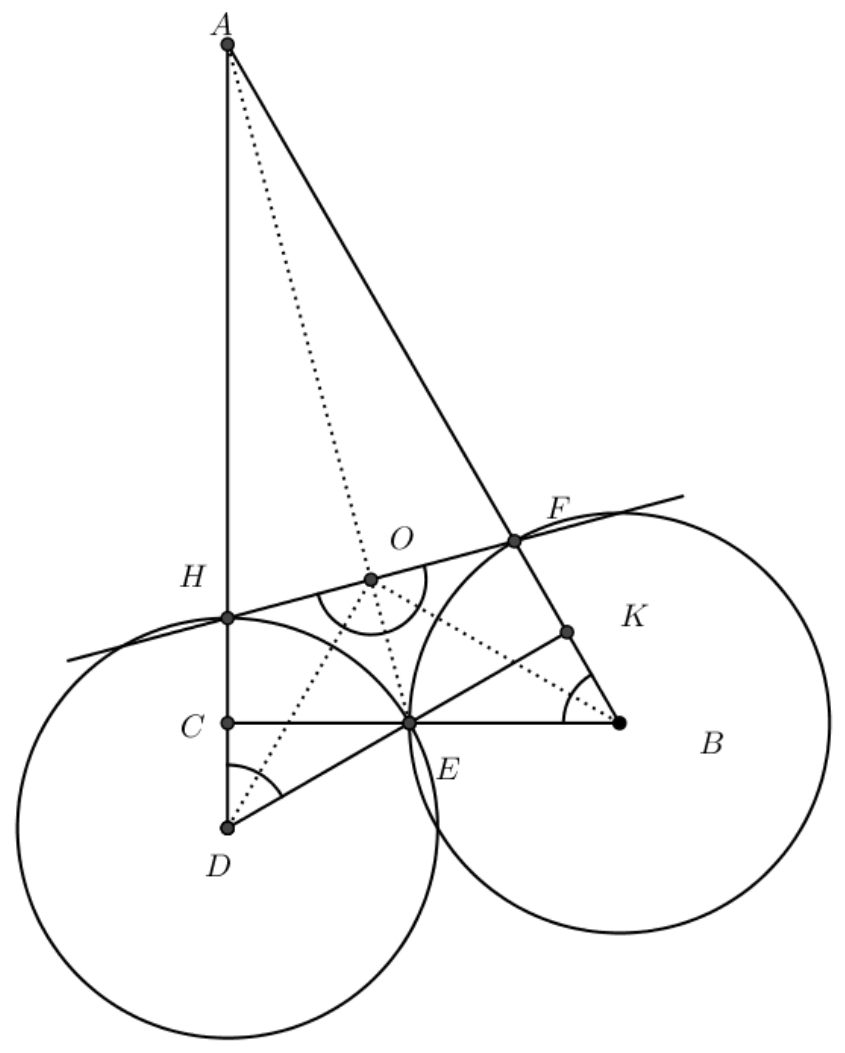

Figura 4.6: Justificativa - Quadrado e Círculo de Mesma Área - Parte 2

Prova. Da figura 4.6, é possível concluir que os triângulos $H D O$ e EDO são congruentes pelo caso $L A L$, pois $\overline{H D}=\overline{D E}$ (raios do mesmo círculo), $\overline{D O}$ é lado comum aos triângulos e $H \hat{D} O=O \hat{D} E$, já que $\overline{D O}$ é bissetriz do ângulo $H \hat{D} E=A \hat{D} K$, conforme provado acima (proposição 24).

Do mesmo modo, com relação os triângulos $E D O$ e $E B O$, tem-se que $\overline{D O}=$ $\overline{B O}$, pela congruência entre os triângulos $A O D$ e $A O B$ provada acima (proposição 24), $O \hat{D} E=O \hat{B} E$, já que $\overline{B O}$ e $\overline{D O}$ são bissetrizes dos ângulos $\hat{D}$ e $\hat{B}$ de mesma medida e, ainda, $\overline{D E}=\overline{B E}$ (proposição 22). Assim, pelo critério $L A L$, os triângulos $E D O$ e $E B O$ também são congruentes. 
Por último, mostraremos que os triângulos $E B O$ e $F B O$ são congruentes. Os lados $\overline{B F}$ e $\overline{B E}$ são raios do mesmo círculo; $\overline{B O}$ é lado comum aos dois triângulos e, ainda $F \hat{B} O=O \hat{B} E$, pois $\overline{B O}$ é bissetriz do ângulo $\hat{B}$. Consequentemente, os triângulos $E B O$ e $F B O$ também são congruentes.

Portanto, os triângulos $H D O, E D O, E B O$ e $F B O$ são todos congruentes. Daí, segue que $H \hat{O} D=D \hat{O} E=E \hat{O} B=B \hat{O} F$. Determinaremos o valor dos ângulos mencionados.

No triângulo $A E B$, tem-se que o ângulo $B \hat{A} E=\frac{\pi}{12}$ e $A \hat{B} E=\frac{\pi}{3}$. Daí, segue que $A \hat{E} B=O \hat{E} B=\pi-\frac{\pi}{3}-\frac{\pi}{12}=\frac{7 \pi}{12}$. Pela congruência dos triângulos provada anteriormente, tem-se que $D \hat{H} O=O \hat{E} D=O \hat{E} B=B \hat{F} O=\frac{7 \pi}{12}$.

Por conseguinte, nos triângulos $H D O, E D O, E B O$ e $F B O$, os ângulos do vértice $O$ têm medida $\pi-\frac{7 \pi}{12}-\frac{\pi}{6}=\frac{\pi}{4}$, donde se conclui que $H \hat{O} F=$ $H \hat{O} D+D \hat{O} E+E \hat{O} B+B \hat{O} F$ mede $\pi$. Logo, os pontos $H, O$ e $F$ são colineares, como queríamos demonstrar.

Proposição 26 Os pontos $E, F, G$ e $H$ são os vértices de um quadrado euclideano.

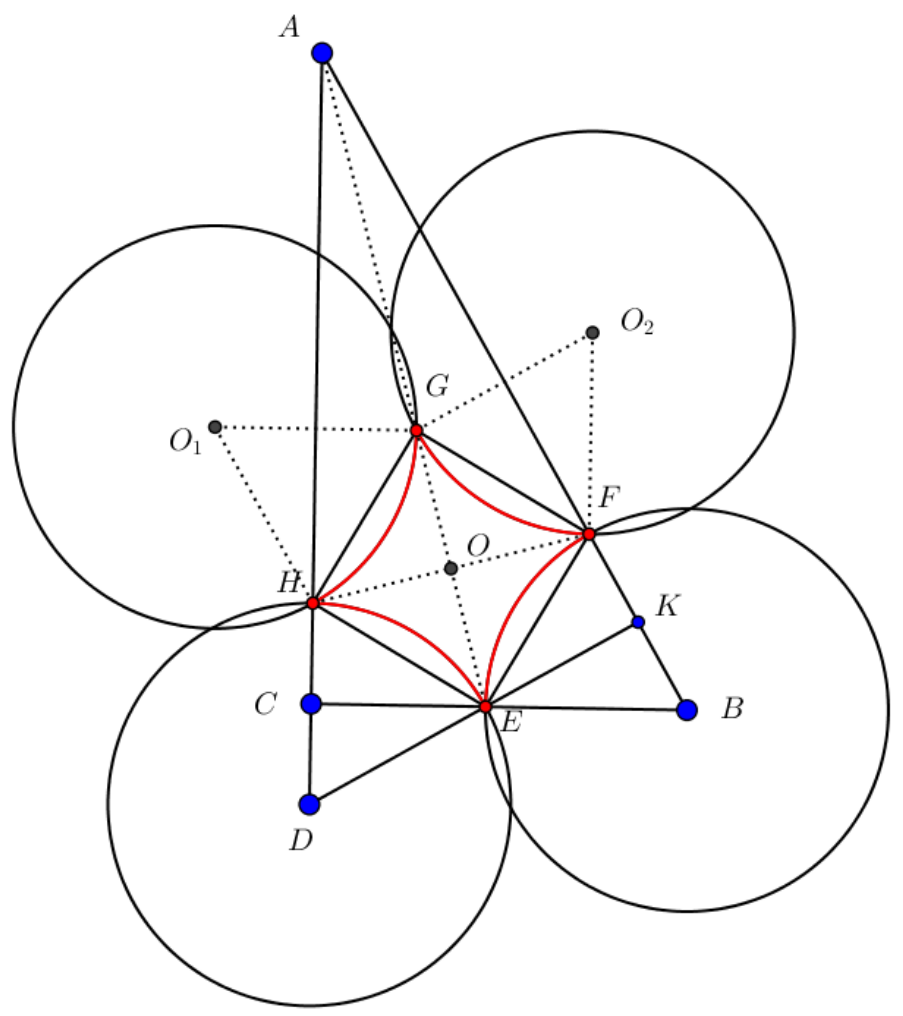

Figura 4.7: Justificativa - Construção do Quadrado e do Círculo de Mesma Área - Parte 3 
Prova. Da figura 4.7, é possível verificar que os segmentos euclideanos $\overline{E F}, \overline{F G}$, $\overline{G H}$ e $\overline{H E}$ têm a mesma medida. Para mostrar tal fato, basta observar que, em virtude da simetria existente na construção, os círculos que representam as retas hiperbólicas têm o mesmo raio e cada um dos segmentos é lado de um triângulo em que os outros dois lados são raios e que os ângulos opostos aos segmentos têm a mesma medida $\left(\frac{\pi}{3}\right)$. Assim, pelo critério $L A L$, os triângulos $O_{1} H G, O_{2} G F, B F E$ e $D E H$ são congruentes, donde segue que $\overline{E F}=\overline{F G}=\overline{G H}=\overline{H E}$.

Ademais, afirmamos que o ângulo euclideano $H \hat{E} F$ mede $\frac{\pi}{2}$. De fato, o triângulo $D E H$ é equilátero, pois $\overline{D E}=\overline{D H}$ (raios do mesmo círculo) e $H \hat{D} E$ mede $\frac{\pi}{3}$. Por motivo análogo, $B E F$ também é equilátero.

No triângulo $D E H$, tem-se que $\overline{E C}$ é altura, e portanto, também é bissetriz. Logo, $C \hat{E} H=\frac{\pi}{6}$. Como $B E F$ é equilátero, segue que $B \hat{E} F=\frac{\pi}{3}$. Mas, $B \hat{E} F+C \hat{E} H+F \hat{E} H=\pi$ e, portanto $F \hat{E} H$ mede $\frac{\pi}{2}$. Como os lados de EFGH são todos congruentes e tal quadrilátero euclideano tem um ângulo de medida $\frac{\pi}{2}$, conclui-se que $E F G H$ é um quadrado.

Como EFGH é um quadrado euclideano, tem-se que suas diagonais $\overline{E G}$ e $\overline{F H}$ são perpendiculares e se intersectam no respectivo ponto médio. Segue, do provado anteriormente (proposição 25), que as diagonais se intersectam em $O$, incentro dos triângulos.

Proposição 27 O ponto $G$ está sobre a reta $\overleftrightarrow{A E}$

Prova. Na figura 4.7, observa-se que o triângulo $A H F$ é isósceles, já que $\overline{A B}=\overline{A D}$ e $\overline{B F}=\overline{D H}$ (raios de círculos), donde se conclui que $\overline{A H}=$ $\overline{A D}-\overline{D H}=\overline{A B}-\overline{B F}=\overline{A F}$.

Como $\hat{A}$ é um dos ângulos de tal triângulo e $\overleftrightarrow{A E}$ é bissetriz, tem-se que $\overleftrightarrow{A E}$ é perpendicular a $\overline{H F}$. Mas $\overline{G E}$ também é perpendicular a $\overline{H F}$, já que os dois segmentos são diagonais do quadrado euclideano EFGH. Como existe apenas uma reta que passa por $E$ e é perpendicular a $\overline{H F}$, conclui-se que $G$ está sobre $\overleftrightarrow{A E}$

Proposição $28 O$ segmento $\overline{O J}$ é perpendicular a $\overline{B J}$, raio do círculo que contém a reta hiperbólica que passa por $E$ e $F$.

Prova. $J$ é o ponto de interseção do círculo centrado em $B$ com o círculo de diâmetro $\overline{O B}$ (figura 4.4). Portanto, $\overline{O J}$ e $\overline{B J}$ são perpendiculares, já que o ângulo inscrito $O \hat{J} B$ tem medida $\frac{\pi}{2}$, correspondente à metade da do arco $\widehat{O B}=\pi$. Logo, o círculo de centro $O$ e raio $\overline{O J}$ é perpendicular ao de centro $B$ e raio $\overline{B J}$. 
O círculo de centro $O$ e raio $\overline{O J}$ será tomado como o disco de Poincaré, já que o arco de círculo centrado em $B$ e que passa pelos pontos $E$ e $F$ é perpendicular ao disco referido e representará uma reta hiperbólica.

Pela simetria existente na construção, conclui-se que os demais círculos que contém os segmentos de retas hiperbólicas também são perpendiculares ao círculo de centro $O$ e raio $\overline{O J}$.

Propoposição 29 Os ângulos do quadrilátero hiperbólico EFGH têm medida $\frac{\pi}{6}$.

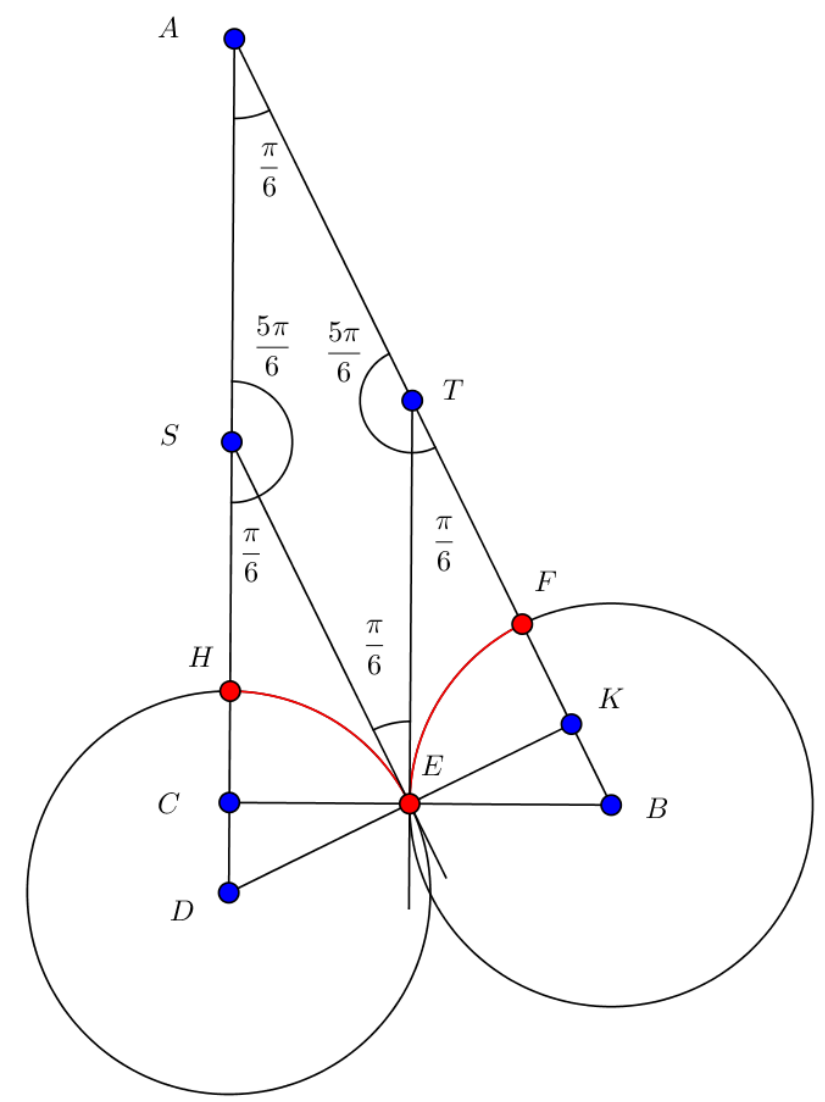

Figura 4.8: Justificativa - Construção do Quadrado e do Círculo de Mesma Área - Parte 4

Prova. Na figura 4.8, acima, a reta euclideana $\overleftrightarrow{E S}$ é tangente ao círculo com centro $D$, que contém a reta hiperbólica que passa por $E$ e $H$, donde se conclui que $\overline{E S} \perp \overline{D K}$ e, por via de consequência, $\overline{E S}$ é paralelo a $\overline{A K}$ e $K \hat{A} D=E \hat{S} D=\frac{\pi}{6}$.

Do mesmo modo, como a e-reta $\overleftrightarrow{E T}$ é tangente ao círculo que contém a reta hiperbólica que passa por $\mathrm{E}$ e $\mathrm{T}, \overline{E T} \perp \overline{B C}$. Logo, $\overline{E T}$ é paralelo a $\overline{A C}$ e $B \hat{T} E=B \hat{A} C=\frac{\pi}{6}$.

Daí segue que, no quadrilátero euclideano ETAS (figura), há um ângulo de medida $\frac{\pi}{6}$ e dois ângulos de $\frac{5 \pi}{6}$. Uma vez que os ângulos do quadrilátero devem 
somar $2 \pi$, tem-se que o ângulo formado entre as retas tangentes $\overleftrightarrow{E T}$ e $\overleftrightarrow{E S}$ tem medida $\frac{\pi}{6}$. Ora, mas este ângulo é o mesmo formado entre as retas hiperbólicas que passam por $E$ e $F$ e $E$ e $H$ e tem medida $\frac{\pi}{6}$, como queríamos demonstrar. Mostraremos, em seguida, que o ângulo formado pelos demais pares de retas hiperbólicas também mede $\frac{\pi}{6}$.

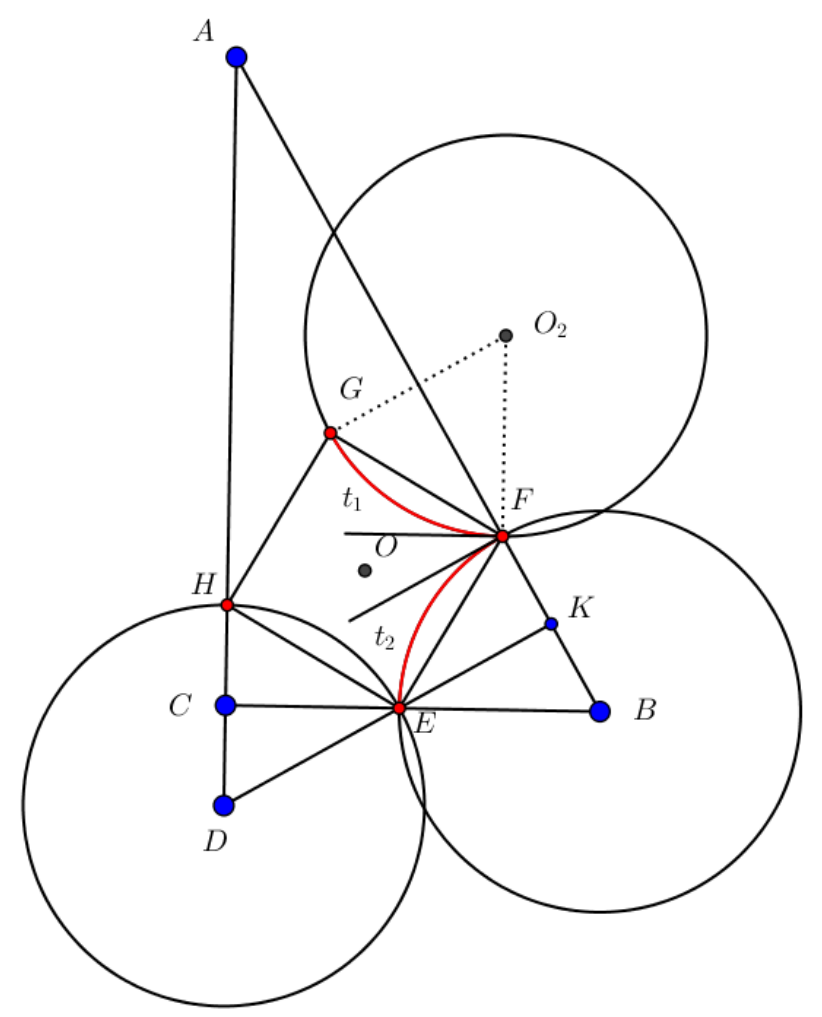

Figura 4.9: Justificativa - Construção do Quadrado e do Círculo de Mesma Área - Parte 5

Na figura 4.9, tem-se que, como EFGH é um quadrado euclideano (proposição 26), $\overline{E H}$ é paralelo a $\overline{F G}$. Pela simetria existente na construção, sabe-se que o triângulo $B E F$ é equilátero, do mesmo modo que o triângulo $O_{2} G F$. Logo, o ângulo $O_{2} \hat{F} G$ mede $\frac{\pi}{3}$.

O ângulo $E \hat{H} D$ mede $\frac{\pi}{3}$, pois $E D H$ é congruente a $E F B$ e também equilátero, por construção. Por conseguinte, o e-segmento $\overline{A C}$ é paralelo ao raio $\overline{O_{2} F}$, já que formam ângulos de mesma medida com os e-segmentos paralelos $\overline{E H}$ e $\overline{F G}$, respectivamente.

Como $\overline{O_{2} F}$ é perpendicular a $t_{1}$, reta tangente ao círculo de centro $O_{2}$ em $F$, e $\overline{A C} \perp \overline{B C}$, segue, do paralelismo provado acima, que $t_{1}$ é paralela a $\overline{B C}$.

A reta $t_{2}$ é tangente ao círculo de centro $B$ no ponto $F$ e, assim, é perpendicular ao raio $\overline{B F}$. Daí, segue que, como $\overline{B F} \perp \overline{D K}, t_{2}$ é paralelo a $\overline{D K}$. 
Do exposto nos parágrafos precedentes, deduz-se que o ângulo formado entre as retas hiperbólicas que passam por $E$ e $F$ e $F$ e $G$ nada mais é do que o ângulo agudo formado entre as retas $\overline{B C}$ e $\overline{D K}$. Deste modo, como as retas mencionadas intersectam-se em $E$, vê-se que $C \hat{E} D$ tem a mesma medida do ângulo formado entre as retas hiperbólicas $\overleftrightarrow{E F}$ e $\overleftrightarrow{F G}$.

No triângulo $C E D$, tem-se que o ângulo $\hat{C}$ tem medida $\frac{\pi}{2}$ e o ângulo $\hat{D}$ mede $\frac{\pi}{3}$, pela hipótese da construção. Portanto, o ângulo $\hat{E}=C \hat{E} D=\frac{\pi}{6}$ Assim, o ângulo entre as retas hiperbólicas $\overleftrightarrow{E F}$ e $\overleftrightarrow{F G}$ mede $\frac{\pi}{6}$, como queríamos demonstrar.

Pela simetria existente na construção em relação à reta $\overleftrightarrow{H F}$ e pelas proposições provadas acima, que garantem a existência de simetria também com relação à reta $\overleftrightarrow{A E}$, conclui-se que os demais ângulos também medem $\frac{\pi}{6}$. Observe-se que, como $\overleftrightarrow{A E}$ e $\overleftrightarrow{H F}$, passam por $O$, centro do disco de Poincaré, a reflexão em relação às referidas retas mantém as propriedades da inversão circular, dentre as quais a preservação de ângulos.

As proposições provadas acima garantem a validade da construção euclideana do quadrado hiperbólico com o ângulo interno pretendido $\frac{\pi}{6}$. Quanto ao círculo de mesma área, sua validade decorre pelas operações realizadas anteriormente, pelas quais obtivemos que o seu raio teria medida euclideana equivalente à metade do comprimento euclideano do raio do disco de Poincaré $(\overline{O J})$, tomado como unidade.

A construção do quadrado hiperbólico pode ser realizada para qualquer ângulo interno construtível. Para verificar tal fato, basta observar que algumas das propriedades provadas acima não dependeram da medida do ângulo considerado e mesmo aquelas em que foram utilizadas medidas de ângulo podem ser provadas considerando um valor genérico de ângulo $\alpha$; é o que se verifica nas figuras abaixo.

Considerando os ângulos marcados na figura 4.10, é possível verificar as mesmas congruências e semelhanças de triângulos verificadas nas demonstrações das proposições 22, 23 e 24, de modo que são válidas as conclusões de que $\overline{B E}=\overline{D E}$ e de que $\overline{A E}$ é bissetriz do ângulo $\hat{A}$ dos triângulos $A B C$ e $A D K$.

$\mathrm{Na}$ figura 4.11, é possível verificar que os triângulos $O A B$ e $O A D$ são congruentes pelo caso $L A L$, já que $\overline{A B}=\overline{A D}, B \hat{A} O=O \hat{A} D=\frac{\alpha}{2}$ e $\overline{A O}$ é lado comum aos dois triângulos euclideanos. Portanto, do mesmo modo, $\overline{D O}=\overline{B O}$.

Tendo em vista os ângulos marcados na figura 4.11, que $\overline{D H}=\overline{D E}=$ $\overline{B E}=\overline{B F}$ e que $\overline{B O}=\overline{D O}$, conclui-se que, da mesma forma que na prova da proposição 25, que os triângulos $H D O, E D O, E B O$ e $F B O$ são todos 


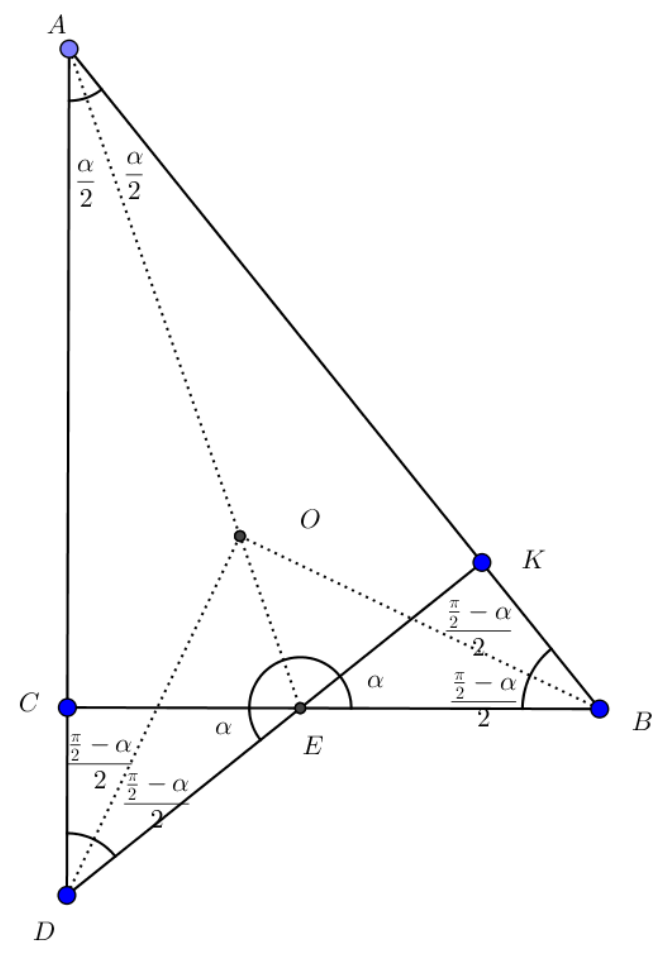

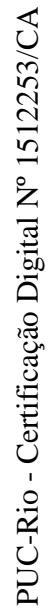

Figura 4.10: Ilustração para a prova da validade das proposições 22, 23 e 24 para ângulos em geral

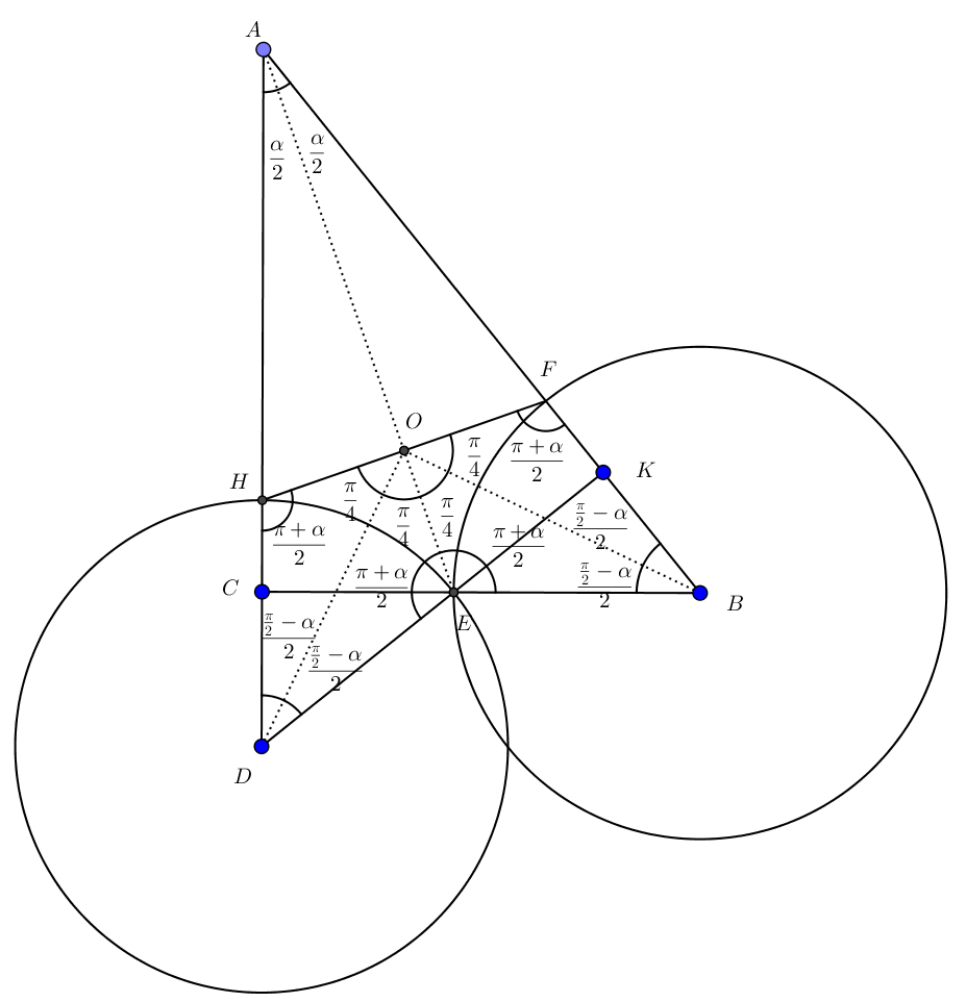

Figura 4.11: Ilustração para a prova da validade da proposição 25 para um ângulo qualquer 
congruentes.

O triângulo $A E B$ tem dois ângulos internos de medida $\frac{\alpha}{2}$ e $\frac{\pi}{2}-\alpha$, respectivamente. Logo, podemos obter a medida do ângulo $A \hat{E} B=O \hat{E} B$ :

$$
\frac{\alpha}{2}+\frac{\pi}{2}-\alpha+O \hat{E} B=\pi \Rightarrow O \hat{E} B=\frac{\pi+\alpha}{2}
$$

Pela congruência dos triângulos $H D O, E D O, E B O$ e $F B O$, foram marcados todos os ângulos que estão na figura. Assim, conclui-se que, analogamente ao já provado acima, que $H \hat{O} D=D \hat{O} E=E \hat{O} B=B \hat{O} F=\frac{\pi}{4}$, donde segue que o ângulo formado entre $\overline{H O}$ e $\overline{O F}$ tem medida $\pi$. Logo, os pontos $H, O$ e $F$ são colineares.

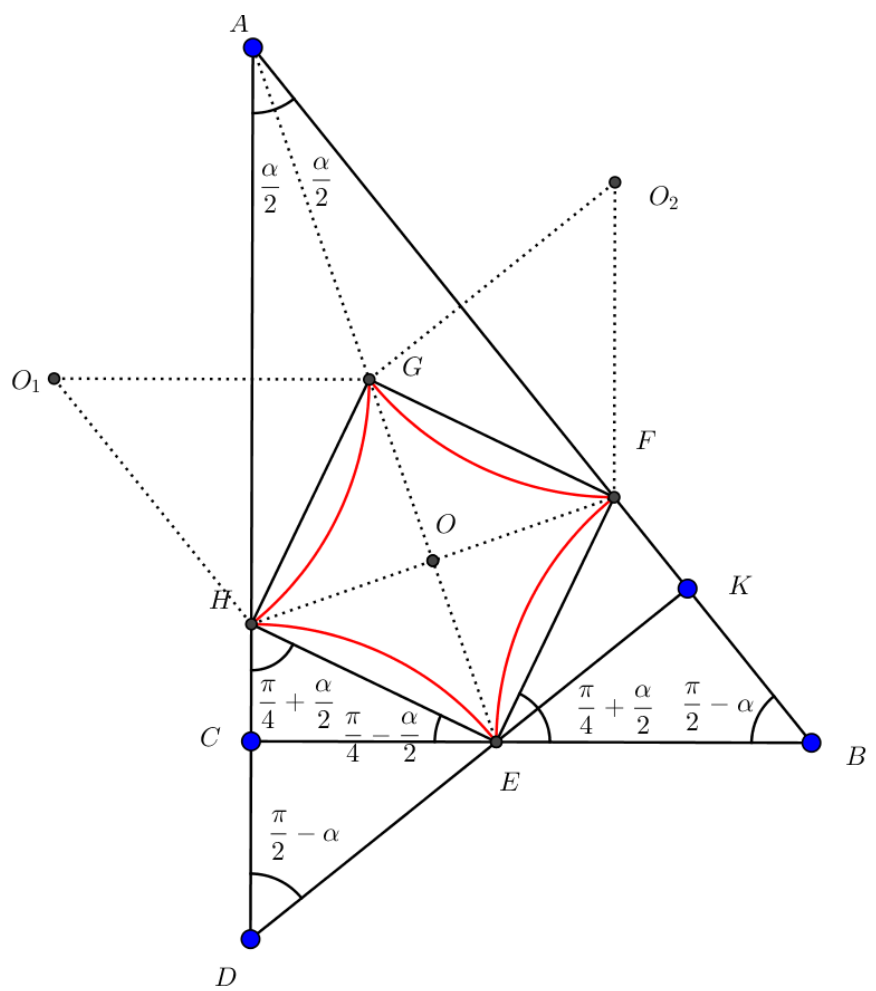

Figura 4.12: Ilustração para a prova da proposição 26 para ângulos quaisquer.

Na figura 4.12, é possível verificar que são válidos os mesmos fatos provados quando da demonstração da proposição 26: pela simetria da construção, os triângulos $H E D, E F B, G H O_{1}$ e $F G O_{2}$ são todos congruentes e isósceles, já que os lados de tais triângulos são raios de círculo com mesma medida e o ângulo formado por estes lados são congruentes, com medida $\frac{\pi}{2}-\alpha$. A única ressalva a ser feita é que, no caso geral, os triângulos referidos são isósceles e não equiláteros. A congruência dos triângulos referidos garante que $\overline{E F}=\overline{F G}=\overline{G H}=\overline{H E}$.

Como o triângulo FEB é isósceles, foi calculada a medida do ângulo 
$F \hat{E} B$ :

$$
\frac{\pi}{2}-\alpha+2 . F \hat{E} B=\pi \Rightarrow F \hat{E} B=\frac{\pi}{4}+\frac{\alpha}{2}
$$

Como os triângulos $F E B$ e $H E D$ são congruentes, segue que $E \hat{H} D=$ $\frac{\pi}{4}+\frac{\alpha}{2}$. Deste modo, no triângulo retângulo $C H E$, vamos obter a medida do ângulo $C \hat{E} H$ :

$$
\frac{\pi}{4}+\frac{\alpha}{2}+H \hat{E} C=\frac{\pi}{2} \Rightarrow H \hat{E} C=\frac{\pi}{4}-\frac{\alpha}{2}
$$

Mas $H \hat{E} C+H \hat{E} F+F \hat{E} B=\pi$. Daí, segue que o ângulo $H \hat{E} F$ tem medida $\frac{\pi}{2}$. Portanto, como o quadrilátero euclideano EFGH tem os quatro lados congruentes e tem um ângulo interno que mede $\frac{\pi}{2}$, conclui-se que $E F G H$ é um quadrado euclideano.

As proposições 27 e 28, são válidas para a construção realizada para um ângulo qualquer, uma vez que as demonstrações destas não dependeram, de forma direta, da medida do ângulo considerado.

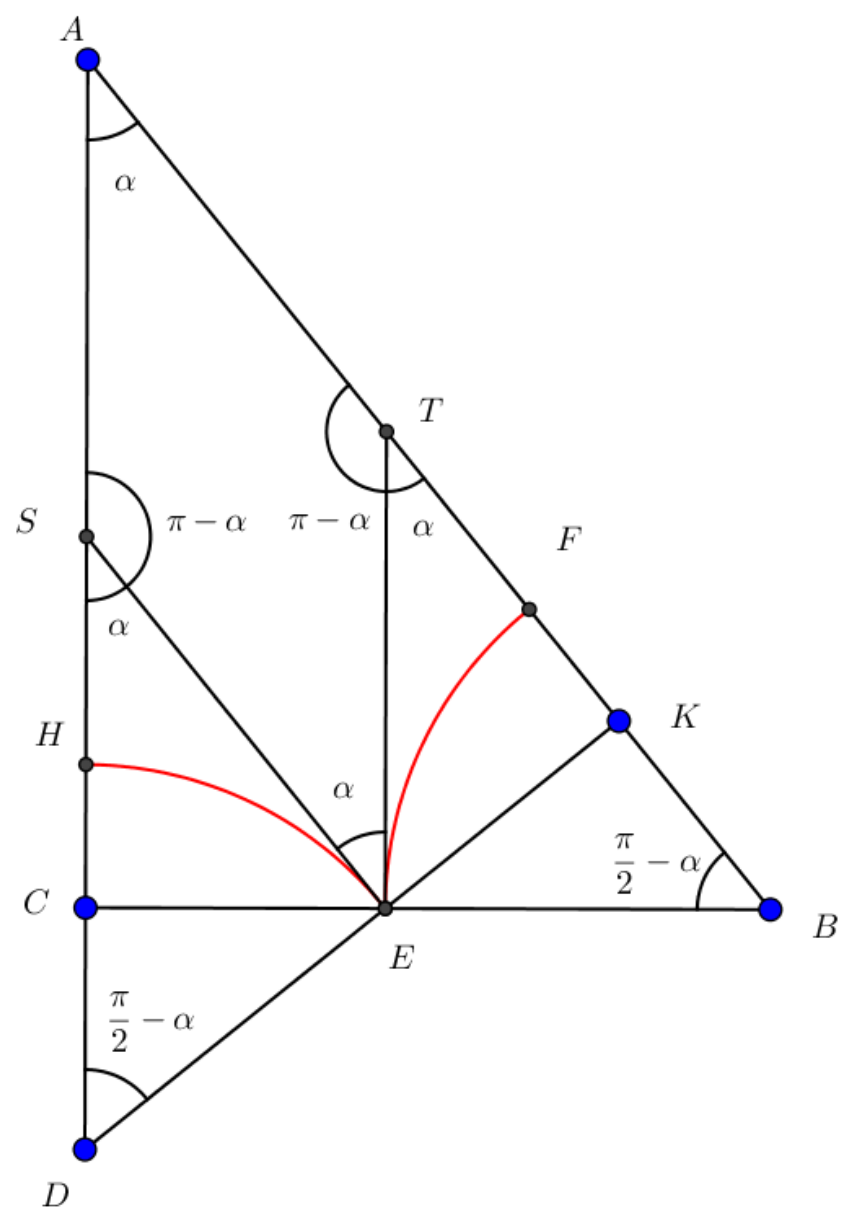

Figura 4.13: Primeira ilustração para a prova da proposição 29 para um ângulo qualquer

Da figura 4.13, verificam-se os mesmos fatos já provados da proposição 
29: as retas $\overleftrightarrow{E T}$ e $\overleftrightarrow{E S}$, tangentes, no ponto $E$, aos círculos que contém os pontos $E$ e $F$ e $E$ e $H$, são perpendiculares aos raios $\overline{B E}$ e $\overline{E D}$. Consequentemente, o quadrilátero euclideano ETAS tem os ângulos marcados na figura, donde segue que o ângulo formado pelas retas hiperbólicas $\overleftrightarrow{E F}$ e $\overleftrightarrow{F H}$ mede $\alpha$.

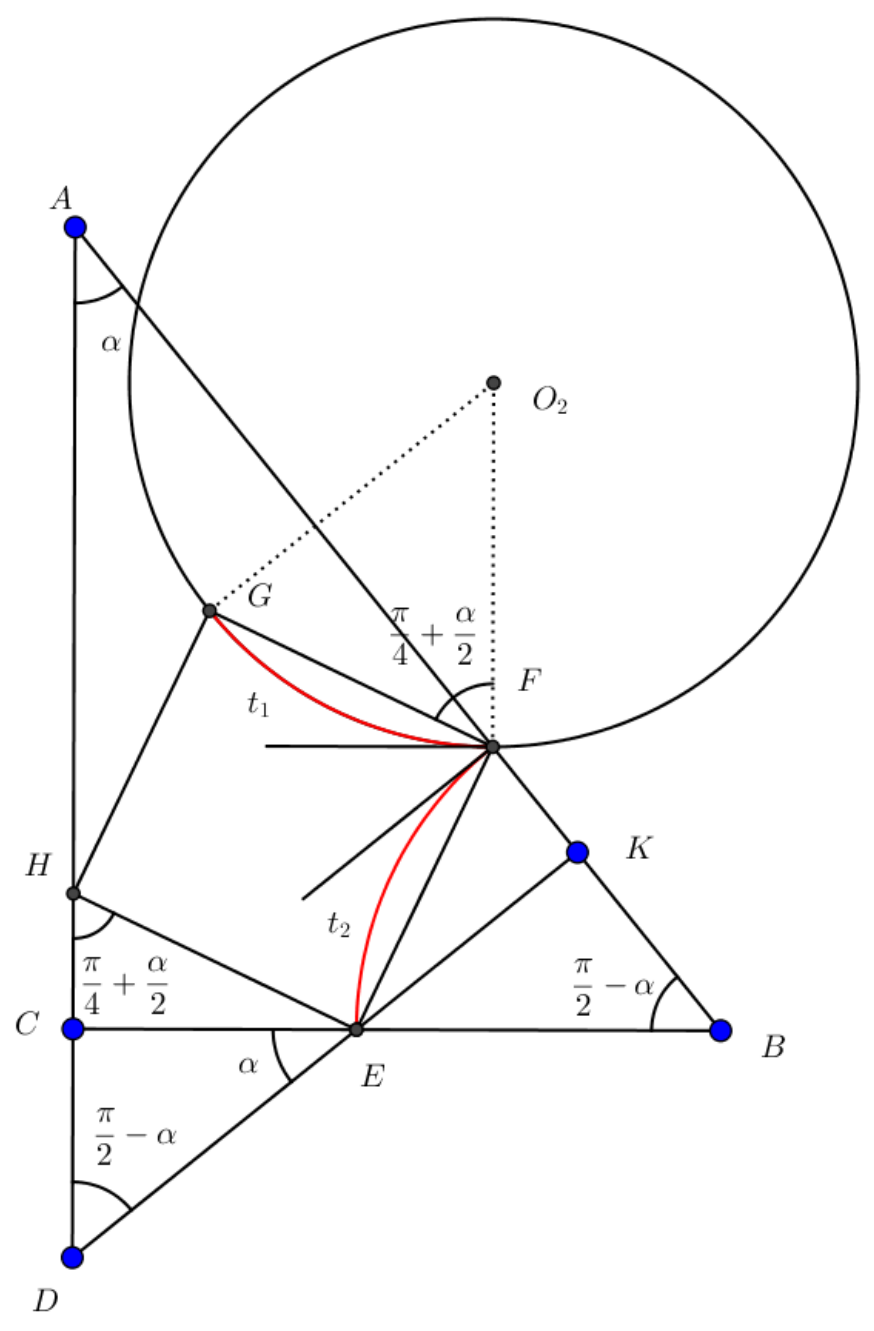

Figura 4.14: Primeira ilustração para a prova da proposição 29 para um ângulo qualquer

Por fim, na figura 4.14, abaixo, apesar da modificação do ângulo, são válidas as mesmas conclusões da segunda parte da demonstração da proposição 29: $\overline{E H}$ é paralelo a $\overline{F G}$, pois são lados opostos do quadrado euclideano $E F G H$. Assim, como $E \hat{H} C=G \hat{F} O_{2}$, segue que $\overline{O_{2} F}$ é paralelo a $\overline{A C}$. Como $\overline{A C} \perp \overline{B C}$ e $\overline{O_{2} F}$ é perpendicular à reta $t_{1}$, tangente ao círculo de centro $O_{2}$ no ponto $F$, segue que $t_{1}$ é paralela a $\overline{B C}$.

Ademais, como $t_{2}$ é tangente ao círculo de centro $B$ e raio $\overline{B F}$ (não mostrado na figura), tem-se que $t_{2} \perp \overline{B F}$. Logo, $t_{2}$ é paralela a $\overline{D K}$.

Deste modo, o ângulo formado entre $t_{1}$ e $t_{2}$ é o mesmo formado entre $\overline{B C}$ e $\overline{D K}$, que se intersectam no ponto $E$. Portanto, o ângulo formado entre 
as retas hiperbólicas $\overleftrightarrow{E F}$ e $\overleftrightarrow{F G}$ é congruente ao ângulo $C \hat{E} D$, que mede $\alpha$.

Pela simetria da construção, conclui-se, do mesmo modo, que todos os ângulos do quadrilátero hiperbólico $E F G H$ têm medida $\alpha$, o que encerra a prova da validade da construção para um quadrado hiperbólico $E F G H$ com ângulo interno qualquer construtível.

Conseguimos verificar que a construção do quadrado hiperbólico, realizada acima, é válida para qualquer ângulo interno construtível. No entanto, cabe indagar: é sempre possível construir, com régua e compasso (euclideanos), no plano hiperbólico, o círculo que tem mesma área que o quadrado? Caso a resposta à primeira questão seja negativa, quais seriam as condições necessárias e suficientes para que seja possível construir um círculo e um quadrado de mesma área no plano hiperbólico?

É relevante destacar, ainda, que as duas figuras - quadrado e círculo - foram construídas simultaneamente, de modo que não foi realizada, propriamente, uma quadratura de círculo. Por este motivo, também é possível questionar: existe um método, para o plano hiperbólico, que permita construir, a partir do quadrado, o círculo de mesma área, ou a partir do círculo, o quadrado de mesma área?

Para fixar ideias, apresentaremos alguns exemplos, mas antes será necessário introduzir uma fórmula que relaciona o comprimento euclideano do raio do círculo $x$ e a medida do ângulo do quadrado $\sigma$.

Começamos notando que a fórmula da área do círculo pode ser reescrita como $2 \pi \cdot(\cosh r-1)$. De fato,

$$
\begin{gathered}
2 \pi \cdot(\cosh r-1)=2 \pi \cdot\left(\frac{e^{r}+e^{-r}}{2}-1\right)=2 \pi \cdot\left(\frac{e^{r}-2+e^{-r}}{2}\right)= \\
=4 \pi \cdot\left(\frac{e^{r}-2+e^{-r}}{4}\right)=4 \pi \cdot\left(\frac{e^{\frac{r}{2}}-e^{-\frac{r}{2}}}{2}\right)^{2}=4 \pi \sinh ^{2} \frac{r}{2}
\end{gathered}
$$

A fórmula da área do círculo, reescrita da forma acima exposta, será um grande auxílio na prova da proposição a seguir.

Proposição 30 Considere um círculo hiperbólico, no disco de Poincaré, com centro em $O$, centro da absoluta, raio hiperbólico $r$ e raio euclideano $x$. Seja $\sigma$ o ângulo interno do quadrado hiperbólico que tem mesma área que o círculo. Nestas condições, tem-se que

$$
x=\sqrt{\frac{\frac{\pi}{2}-\sigma}{\frac{3 \pi}{2}-\sigma}}
$$


Prova. O quadrado e o círculo, por hipótese, têm a mesma área. Assim,

$$
2 \pi-4 \sigma=2 \pi(\cosh r-1)
$$

Mas, pela proposição 18, sabe-se que $\cosh r=\frac{1+x^{2}}{1-x^{2}}$. Efetuando a substituição do segundo membro da igualdade em $(*)$, obtemos

$$
2 \pi-4 \sigma=2 \pi \cdot\left(\frac{1+x^{2}}{1-x^{2}}-1\right)
$$

Dividindo os dois membros da igualdade acima por 4, chegamos a

$$
\begin{aligned}
\frac{\pi}{2}-\sigma & =\frac{\pi}{2} \cdot\left(\frac{1+x^{2}}{1-x^{2}}\right)-\frac{\pi}{2} \\
\left(\frac{\pi}{2}-\sigma\right) \cdot\left(1-x^{2}\right) & =\frac{\pi}{2} \cdot\left(1+x^{2}\right)-\frac{\pi}{2} \cdot\left(1-x^{2}\right) \\
\frac{\pi}{2}-\sigma-\left(\frac{\pi}{2}-\sigma\right) \cdot x^{2} & =\pi x^{2} \\
\frac{\pi}{2}-\sigma & =\left(\frac{\left.\frac{3 \pi}{2}-\sigma\right)}{2} x^{2}\right. \\
x & =\sqrt{\frac{\frac{\pi}{2}-\sigma}{\frac{3 \pi}{2}-\sigma}}
\end{aligned}
$$

Na sequência, apresentaremos os exemplos anteriormente referidos:

\section{Exemplo $1 \sigma=\frac{\pi}{4}$.}

Neste primeiro exemplo, estamos partindo da medida do ângulo $\sigma$, já mencionada. Neste caso, tem-se que a medida euclideana do raio do círculo será dada por

$$
x=\sqrt{\frac{\frac{\pi}{2}-\frac{\pi}{4}}{\frac{3 \pi}{2}-\frac{\pi}{4}}}=\sqrt{\frac{\frac{\pi}{4}}{\frac{5 \pi}{4}}}=\frac{1}{\sqrt{5}}
$$

Observamos, deste modo, que, para $\sigma=\frac{\pi}{4}$, a construção do quadrado e do círculo de mesma área é possível. A única diferença é que, com o ângulo assinalado, a construção do círculo será mais trabalhosa, pois, tomando o raio euclideano da absoluta como unidade, será necessário obter o comprimento $\sqrt{5}$ 
para, posteriormente, realizar a construção do segmento de medida $\frac{1}{\sqrt{5}}$, com utilização dos métodos vistos na demonstração do teorema 1.

Faz-se necessário um último comentário. A medida hiperbólica do raio do círculo, no exemplo ora estudado, será dada por

$$
r=\ln \left(\frac{1+\frac{1}{\sqrt{5}}}{1-\frac{1}{\sqrt{5}}}\right)=\ln \left(\frac{\sqrt{5}+1}{\sqrt{5}-1}\right)=\ln \left(\frac{6+2 \sqrt{5}}{4}\right)=\ln \left(\frac{3+\sqrt{5}}{2}\right)
$$

É fácil ver que sinh $\left(\ln \left(\frac{3+\sqrt{5}}{2}\right)\right)$ é um comprimento construtível no plano euclideano, pois é um número que é o resultado de operações de corpos e extração de raiz quadrada envolvendo números racionais. Portanto, $r$ pode ser construído no plano hiperbólico (teorema 19), o que ratifica a possibilidade da construção do quadrado e do círculo de mesma área quando o ângulo interno do quadrado é $\sigma=\frac{\pi}{4}$.

Exemplo $2 r=\ln \left(\frac{16+5 \sqrt{7}}{9}\right)$

No segundo exemplo, observamos, primeiramente, que $r$ é construtível no plano hiperbólico, já que sinh $r$ é um número obtido por operações de corpos e extração de raiz quadrada envolvendo números racionais (teorema 19). Obteremos a medida do raio euclideano $x$ :

$$
\begin{gathered}
\ln \left(\frac{16+5 \sqrt{7}}{9}\right)=\ln \left(\frac{1+x}{1-x}\right) \\
\frac{16+5 \sqrt{7}}{9}=\frac{1+x}{1-x} \\
9+9 x=16+5 \sqrt{7}-(16+5 \sqrt{7}) x \\
x=\frac{7+5 \sqrt{7}}{25+5 \sqrt{7}}=\frac{(7+5 \sqrt{7}) \cdot(25-5 \sqrt{7})}{450}=\frac{(7+5 \sqrt{7}) \cdot(5-\sqrt{7})}{90}=\frac{18 \sqrt{7}}{90}=\frac{\sqrt{7}}{5}
\end{gathered}
$$

O raio euclideano do círculo que se pretende construir $x=\frac{\sqrt{7}}{5}$ pode ser construído com régua e compasso em $\mathbb{E}^{2}$. No entanto, como veremos a seguir, o ângulo interno $\sigma$ não é construtível. De fato,

$$
\sqrt{\frac{\frac{\pi}{2}-\sigma}{\frac{3 \pi}{2}-\sigma}}=\frac{\sqrt{7}}{5}
$$




$$
\begin{gathered}
\frac{\pi-2 \sigma}{3 \pi-2 \sigma}=\frac{7}{25} \\
25 \pi-50 \sigma=21 \pi-14 \sigma \Rightarrow \sigma=\frac{4 \pi}{36}=\frac{\pi}{9}
\end{gathered}
$$

Concluímos que o ângulo $\sigma=\frac{\pi}{9}$ não pode ser construído, com régua e compasso, no plano euclideano, com base no teorema 6 (Gauss-Wentzel), pois, caso contrário, seria possível construir um polígono com 18 lados, o que estaria em desacordo com o resultado mencionado. Como o ângulo referido não é construtível em $\mathbb{E}^{2}$, ele também não pode ser construído com régua e compasso no plano hiperbólico.

\section{Exemplo $3 \sigma=\arctan \frac{3}{4}$}

No que diz respeito a este exemplo, cumpre esclarecer que o ângulo $\sigma$ pode ser construído, com régua e compasso, no plano euclideano, uma vez que $\tan \sigma \in \mathbb{Q}$ e, por via de consequência, $\tan \sigma \in E$. Do mesmo modo, o referido ângulo é construtível no plano hiperbólico (teorema 20). Obteremos a medida euclideana do raio do círculo de mesma área $x$ :

$$
x=\sqrt{\frac{\frac{\pi}{2}-\arctan \frac{3}{4}}{\frac{3 \pi}{2}-\arctan \frac{3}{4}}}=\sqrt{\frac{\arctan \frac{4}{3}}{\pi-\arctan \frac{4}{3}}}
$$

Daí segue que

$$
\frac{1}{x^{2}}=\frac{\pi}{\arctan \frac{4}{3}}-1
$$

Tem-se que $x \in E$ se, e somente se, $\frac{1}{x^{2}}$ pode ser construído com régua e compasso no plano euclideano. Mas será que este último comprimento é construtível? Ainda não temos condições de responder a tal pergunta, pois será necessário o desenvolvimento da teoria que será exposta na seção 4.2.

De todo modo, a fórmula provada na proposição 30 sinaliza para a necessidade de obtenção de um valor de $x$ com eliminação de $\pi$, que é um número transcendente e não pode ser construído com régua e compasso. Assim, conclui-se que se $x \in E$, então $\sigma=k . \pi$, com com $k \in E$. Adiante, veremos que, na verdade, devemos ter $k \in \mathbb{Q}$, caso contrário, $\sigma$ não será construtível.

Os questionamentos apontados anteriormente serão analisados, de um ponto de vista mais geral, na próxima seção. 


\section{2}

\section{Condições Necessárias e Suficientes para Construir um Quadrado e um Círculo de Mesma Área no Plano Hiperbólico}

Nesta seção, serão enunciados e demonstrados os teoremas que respondem os questionamentos que encerraram a seção anterior, sobre as condições necessárias e suficientes para que seja possível construir um quadrado e um círculo de mesma área no plano hiperbólico e sobre a existência, ou não, de métodos que, a partir do quadrado, permitam construir o círculo e vice-versa. Esta parte do texto é baseada, principalmente, no artigo [4], publicado em 1995. As construções sintéticas apresentadas adiante foram, em sua maioria, baseadas em [12].

Neste trabalho, apresentamos a Geometria Hiperbólica com base no modelo do disco de Poincaré. Porém, as questões tratadas nesta última seção serão desenvolvidas do ponto de vista sintético.

Começamos observando que, como a área do quadrado é dada por $2 \pi-4 \sigma$, em que $\sigma$ é o ângulo interno, conclui-se que a área de tal polígono é menor ou igual a $2 \pi$ e apenas assume o valor máximo para quadrados com lados infinitos. No que diz respeito aos círculos, como sua área é dada por $2 \pi$.( $\cosh r-1)$, em que $r$ é o raio, esta pode assumir qualquer valor positivo. Portanto, ainda sem fazer menção à questão da construtibilidade, apenas círculos com área inferior a $2 \pi(\cosh r \leqslant 2)$ podem ter um correspondente quadrado de mesma área.

Sobre as figuras apresentadas nesta seção, é relevante fazer um esclarecimento: como o tratamento será sintético, as figuras não serão apresentadas com base no modelo do disco de Poincaré. As retas serão desenhadas como se fossem retas euclideanas, com setas para indicar nas extremidades para indicar os paralelismos existentes. Assim, por exemplo, é apresentada, a seguir, uma figura com retas hiperbólicas paralelas que passam por um ponto $P$, em que são detalhados o ângulo de paralelismo $\rho$ e o segmento de paralelismo $t$.

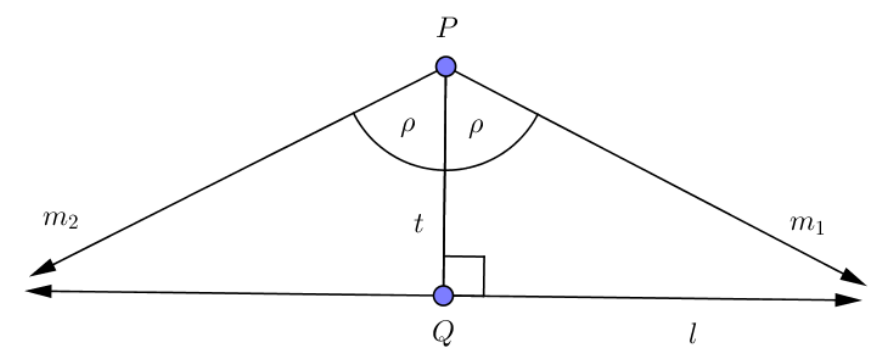

Figura 4.15: Retas paralelas do ponto de vista axiomático

Neste momento, é importante, também, lembrar a bijeção $\Pi: \mathbb{R}_{+} \backslash\{0\} \rightarrow$ 
$\left(0, \frac{\pi}{2}\right)$, que associa a cada comprimento hiperbólico $t$ o seu correspondente ângulo de paralelismo $\Pi(t)$, em que $e^{-t}=\tan \frac{\Pi(t)}{2}$. É necessário recordar, também, que $\Pi$ é decrescente, o que tem grande relevância nas construções que serão realizadas adiante.

Quanto à régua e compasso, ao contrário da abordagem centrada no modelo do disco de Poincaré - em que a régua e o compasso eram combinações ou usos mais sofisticados da régua e do compasso já utilizados na Geometria Euclideana -, no caso da abordagem sintética, estamos lidando com instrumentos intrínsecos que permitem desenhar retas e círculos no plano hiperbólico.

No plano hiperbólico $\left(\mathbb{H}^{2}\right)$, são válidas as construções elementares realizadas no plano euclideano $\left(\mathbb{E}^{2}\right)$, que são as seguintes: (i) adicionar ou subtrair segmentos; (ii) adicionar ou subtrair ângulos; (iii) traçar bissetrizes de ângulos; (iv) traçar perpendiculares por um ponto da reta ou por um ponto exterior a esta e (v) traçar mediatrizes de segmentos. Isto ocorre porque as construções mencionadas não dependem do quinto postulado de Euclides. As diferenças nas construções dizem respeito ao fato de que, na Geometria Hiperbólica, há duas retas paralelas a uma reta dada.

Pelo motivo exposto no parágrafo anterior, serão apresentadas algumas construções que serão necessárias para possibilitar a análise do problema da quadratura do círculo no plano hiperbólico.

Construção 10 Construir uma reta paralela à reta dada l, passando por um ponto $P$.

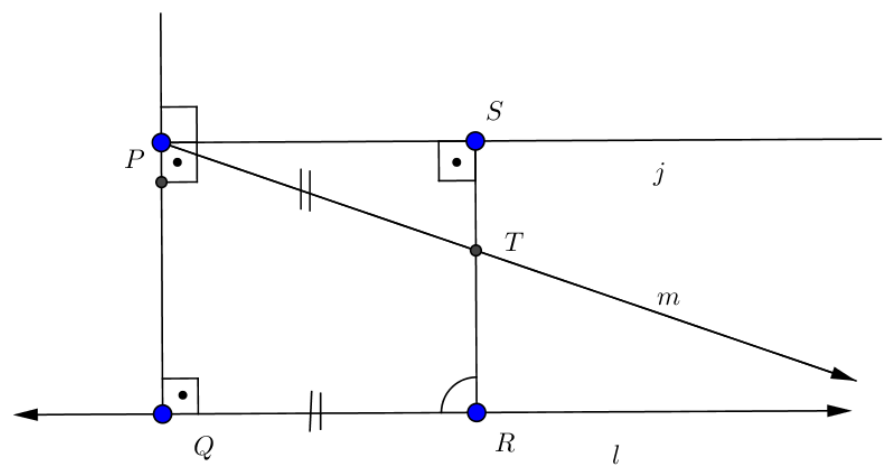

Figura 4.16: Construção de reta paralela do ponto de vista sintético

A construção deve ser realizada da seguinte forma:

1 - por $P$, traçar a perpendicular a $l$, que intersectará tal reta em $Q$;

2 - traçar a semirreta $j$, perpendicular a $\overline{P Q}$, a partir de $P$, na direção da reta paralela desejada; 
3 - por um ponto $R$ qualquer de $l$, traçar uma perpendicular a $j$, que encontrará a semirreta no ponto $S$;

4 - utilizar o compasso para desenhar um círculo com centro em $P$ e raio igual ao comprimento de $\overline{Q R}$. O círculo intersectará o segmento $\overline{R S}$ no ponto $T$;

5 - Traçar $m=\overrightarrow{P T}$, que é a reta paralela procurada.

Justificativa. Na figura 4.17, verifica-se que $P Q R S$ é um quadrilátero com três ângulos retos e um ângulo agudo, conhecido como quadrilátero de Lambert. Com base na primeira ilustração, que contém apenas o quadrilátero $P Q R S$, provaremos, primeiramente, que $\cosh v=\frac{\tanh z}{\tanh u}$.
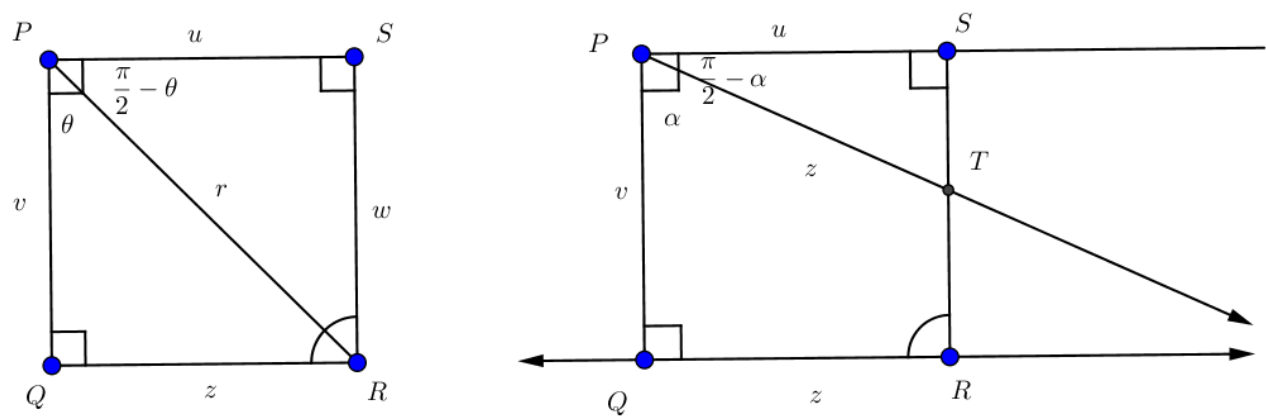

Figura 4.17: Ilustração para justificativa da construção 10

De fato, das relações trigonométricas hiperbólicas aplicadas ao triângulo retângulo (teorema 14), concluímos que

$$
\begin{gathered}
\cos \left(\frac{\pi}{2}-\theta\right)=\frac{\tanh u}{\tanh r} \Rightarrow \tanh u=\cos \left(\frac{\pi}{2}-\theta\right) \cdot \tanh r \Rightarrow \tanh u=\sin \theta \tanh r \\
\Rightarrow \tanh u=\frac{\sinh z}{\sinh r} \cdot \tanh r \Rightarrow \tanh u=\frac{\sinh z}{\cosh r} \Rightarrow \tanh u=\frac{\sinh z}{\cosh z \cosh v} \\
\Rightarrow \tanh u=\frac{\tanh z}{\cosh v} \Rightarrow \cosh v=\frac{\tanh z}{\tanh u} \quad(*)
\end{gathered}
$$

Agora, analisando a segunda ilustração e aplicando uma relação trigonométrica no triângulo PST, chegamos a

$$
\cos \left(\frac{\pi}{2}-\alpha\right)=\frac{\tanh u}{\tanh z} \Rightarrow \sin \alpha=\frac{\tanh u}{\tanh z}
$$

Considerando a relação $(*)$, provada acima, obtemos

$$
\sin \alpha=\frac{1}{\cosh v} \Rightarrow \csc \alpha=\cosh v \quad(* *)
$$


Assim, considerando que $\alpha$ é agudo e que a relação $(* *)$ corresponde à segunda fórmula que relaciona o comprimento de paralelismo com o respectivo ângulo (Teorema 17), conclui-se que $\alpha=\Pi(v)$, donde segue que $l$ e $m$ (figura 4.16) são paralelas, como queríamos demonstrar.

Construção 11 Dado um ângulo agudo $\rho$, formado por duas semirretas que partem de um ponto $A$, construir a reta hiperbólica perpendicular a uma delas e paralela à outra.

Seja $\rho$ o ângulo $B \hat{A} C$ e considere que $C$ é o pé da perpendicular baixada de $B$ à semirreta $\overrightarrow{A C}$. Utilizando a construção 10 , encontramos o ângulo $\Pi(\overline{A B})$. Agora devemos, obter, por construção o ponto $D$ sobre a reta $\overleftrightarrow{B C}$, tal que $C \hat{A} D=\Pi(\overline{A B})$. O segmento $\overline{A D}$ é tal que $\Pi(\overline{A D})=\rho$.

Para finalizar a construção, basta, apenas, utilizar o compasso para construir um círculo de centro $A$ e raio $\overline{A D}$ e a partir do ponto $D_{2}$, interseção deste círculo com a semirreta $\overrightarrow{A C}$, construir uma reta perpendicular a $\overrightarrow{A C}$, que será paralela a $\overleftrightarrow{A B}$ (segunda parte da construção não mostrada na figura).

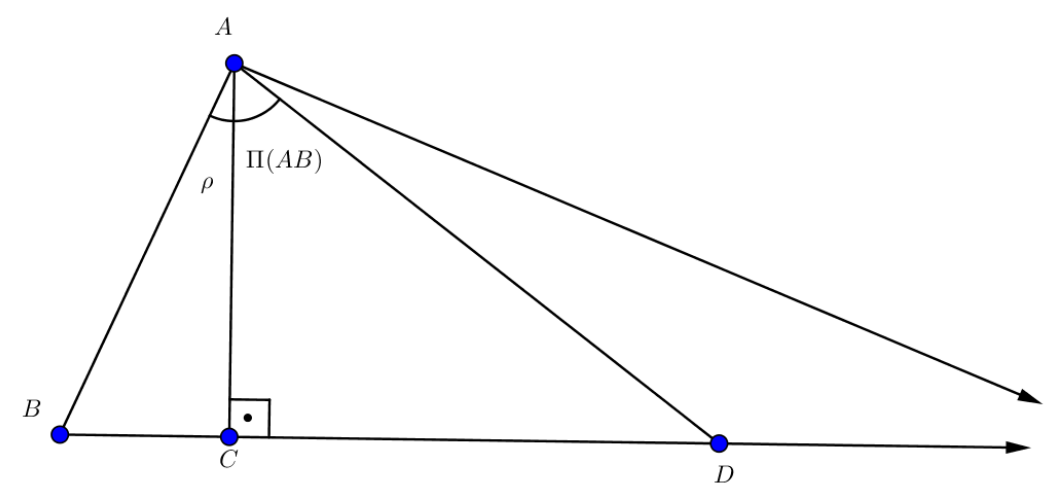

Figura 4.18: Construção do segmento de paralelismo, dado o ângulo de paralelismo $\rho$

Justificativa. Da figura 4.18, verifica-se que $\overline{A B}>\overline{A C}$. Assim, como П é uma função decrescente, segue que $\Pi(\overline{A B})<\Pi(\overline{A C})$. Portanto, o ponto $D$ existe e pode ser obtido por construção. Das relações trigonométricas hiperbólicas dos teoremas 14 e 17 , temos que

$$
\cos \rho=\frac{\tanh \overline{A C}}{\tanh \overline{A B}}=\frac{\tanh \overline{A C}}{\cos \Pi(\overline{A B})}=\frac{\tanh \overline{A C}}{\frac{\tanh \overline{A C}}{\tanh \overline{A D}}}=\tanh \overline{A D}
$$

Como a igualdade $\cos \rho=\tanh \overline{A D}$ corresponde à terceira fórmula apresentada no teorema 17 , segue que $\rho=\Pi(\overline{A D})$, como queríamos demonstrar. 
Construção 12 Construir um triângulo retângulo, dados os dois ângulos internos $\alpha$ e $\beta$ tais que $\alpha+\beta<\frac{\pi}{2}$.

Para realizar a construção do triângulo, deve-se obter, primeiramente, por meio da construção 11 , segmentos de paralelismo $z$ e $t$ tais que $\Pi(z)=\alpha$ e $\Pi(t)=\frac{\pi}{2}-\beta$. Como $\alpha<\frac{\pi}{2}-\beta$ e $\Pi$ é decrescente, segue que $z>t$. Assim, deve-se desenhar, em seguida, um triângulo retângulo de hipotenusa $z$, um cateto $t$ e o outro cateto denotado por $b$. A construção deve ser realizada de forma análoga à da geometria euclideana, por exemplo:

1 - traçar o segmento de medida $t$, cujas extremidades são os pontos $Z$ e $B$;

2 - pelo ponto $Z$, traçar uma perpendicular ao segmento de medida $t$;

3 - utilizar o compasso para desenhar um círculo ao redor de $B$, com raio $z$, que intersectará a perpendicular traçada no ponto $T$.

Após, deve-se desenhar outro triângulo retângulo $\left(A B_{2} C\right)$ em que $\overline{A C}=b$ $b$ e o ângulo $B_{2} \hat{A} C=\alpha$. O outro ângulo de tal triângulo terá medida $\beta$.
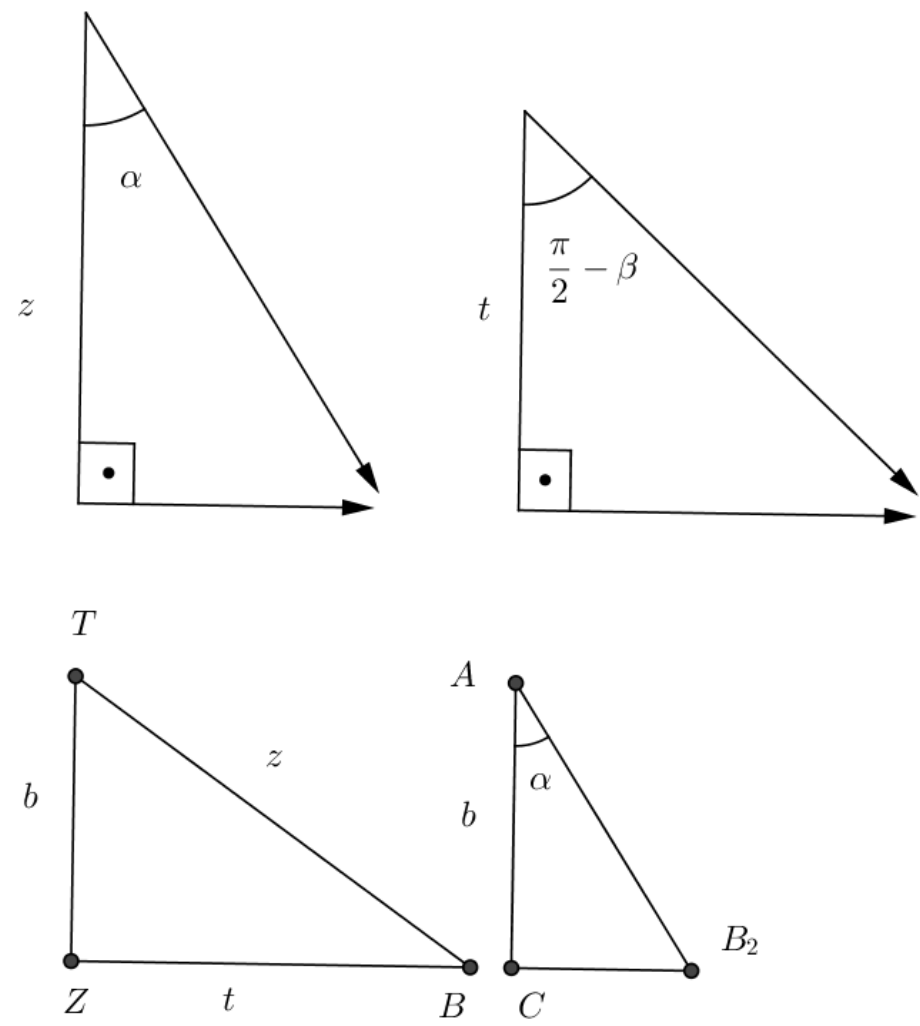

Figura 4.19: Construção dos triângulo retângulo hiperbólico, dados ângulos $\alpha$ e $\beta$

Justificativa. Tendo em vista as relações trigonométricas dos teoremas 14 e 17, 
verificamos, do triângulo $Z T B$, que

$$
\begin{gathered}
\cosh z=\cosh t \cosh b \Rightarrow \cosh b=\frac{\cosh z}{\cosh t} \Rightarrow \cosh b=\frac{\csc \alpha}{\csc \left(\frac{\pi}{2}-\beta\right)} \Rightarrow \\
\Rightarrow \cosh b=\frac{\sin \left(\frac{\pi}{2}-\beta\right)}{\sin \alpha} \Rightarrow \cosh b=\frac{\cos \beta}{\sin \alpha}
\end{gathered}
$$

Agora, aplicando uma das fórmulas do teorema 14 ao triângulo $A B_{2} C$ e denotando o ângulo $A \hat{B}_{2} C$ por $\delta$, chegamos a

$$
\cosh b=\frac{\cos \delta}{\sin \alpha}
$$

Da igualdade entre (1) e (2), segue que $\cos \delta=\cos \beta$. Mas os referidos ângulos são agudos, donde se conclui que $\delta=\beta$. Logo, o triângulo retângulo $A B_{2} C$ tem os ângulos internos agudos $\alpha$ e $\beta$, como queríamos demonstrar.

A seguir, apresentaremos duas construções que relacionam a medida de um segmento $r$ com um ângulo $\theta$, tal que $\tan \theta=2 \sinh \frac{r}{2}$, as quais serão fundamentais para a análise do problema relativo à possibilidade de se construir, no plano hiperbólico, um círculo e um quadrado de mesma área. Antes, porém, faz-se necessário apresentar algumas definições que não foram explicitadas em outra seção deste trabalho, bem como mais uma construção que terá utilidade para justificar uma das construções referentes a $r$ e $\theta$.

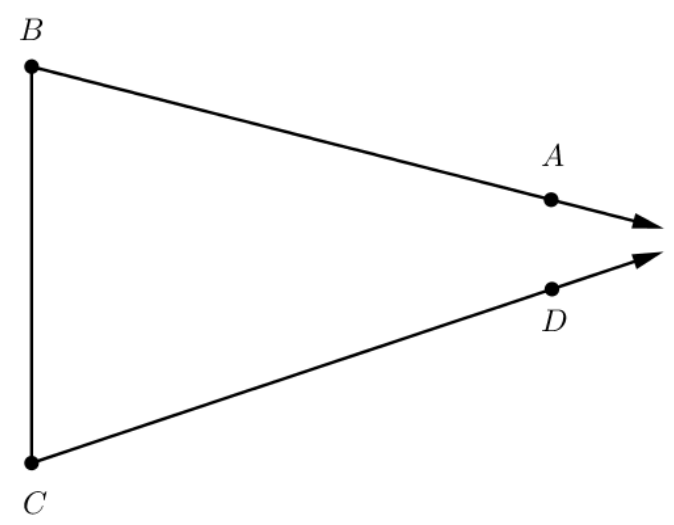

Figura 4.20: Biângulo fechado

Definição 32 (Biângulo fechado) Um biângulo fechado $A B C D$ é uma reunião $\overrightarrow{B A} \cup \overrightarrow{B C} \cup \overrightarrow{C D}$, onde $\overrightarrow{B A}$ e $\overrightarrow{C D}$ são semirretas e $\overrightarrow{B C}$ é segmento tais que $A$ e $D$ estão do mesmo lado de $\overleftrightarrow{B C}$ e que toda semirreta $\overrightarrow{B E}$, que passa 
pelo interior do biângulo, intersecta $\overrightarrow{C D}$ e que toda semirreta $\overrightarrow{C F}$, que passa pelo interior do biângulo, intersecta $\overrightarrow{B A}$.

Um biângulo fechado $A B C D$ é dito isósceles quando se tem $A \hat{B} C=D \hat{C} B$.

Ou seja, um biângulo fechado $A B C D$ é a figura formada pelas semirretas paralelas $\overrightarrow{B A}$ e $\overrightarrow{C D}$ juntamente com o segmento $\overrightarrow{B C}$. A figura acima 4.20 ilustra sinteticamente um biângulo fechado.

A construção exibida e justificada a seguir será importante para obter o segmento $r$ a partir do ângulo $\theta$, tal que $\tan \theta=2 \sinh \frac{r}{2}$.

Construção 13 Construir, a partir de um biângulo fechado qualquer $A B C D$, um biângulo fechado e isósceles $A B C_{2} D$, com $C_{2} \in \overleftrightarrow{C D}$.

Para realizar a construção acima, devemos cumprir os seguintes passos:

1 - construir a bissetriz do ângulo $A \hat{B} C$, que intersectará $\overrightarrow{C D}$ no ponto $E$

2 - construir a bissetriz do ângulo $B \hat{C} D$, que se encontrará com $\overline{B E}$ no ponto $P$;

3 - baixar as perpendiculares $\overline{P Q}, \overline{P S}$ e $\overline{P R}$ a $\overrightarrow{B A}, \overrightarrow{B C}$ e $\overrightarrow{C D}$, respectivamente;

4 - prolongar a reta $\overleftrightarrow{C D}$ no sentido oposto ao do ponto $D$ e utilizar o compasso para construir um círculo com centro $R$ e raio $\overline{Q B}$, que intersectará $\overleftrightarrow{C D}$ no ponto $C_{2}$, do mesmo lado de $\overline{Q R}$ que $B$

5 - traçar o segmento $\overline{B C_{2}}$, obtendo, finalmente, o biângulo fechado e isósceles $A B C_{2} D$.

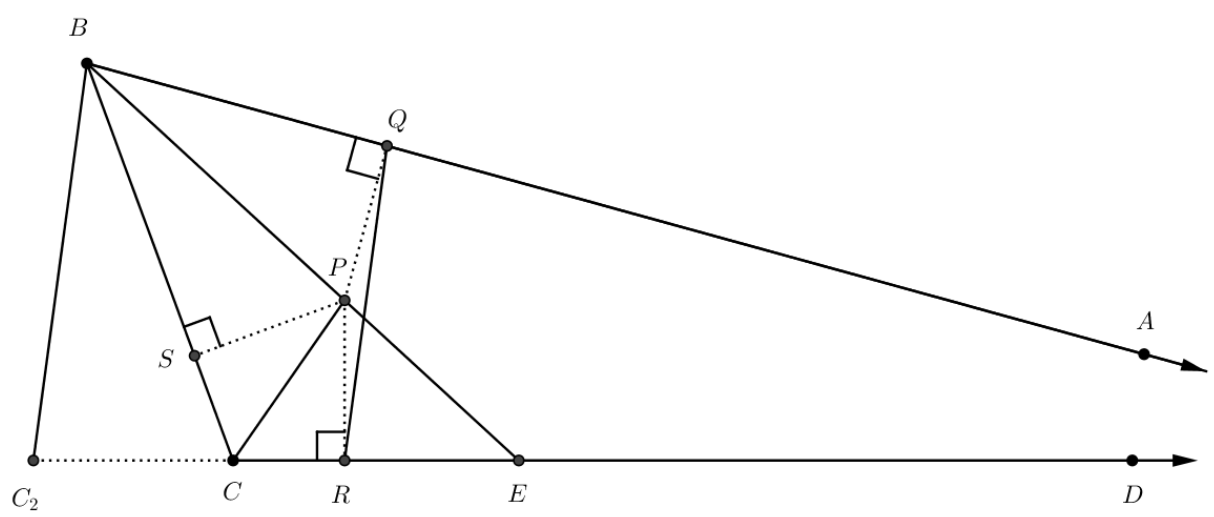

Figura 4.21: Construção do biângulo isósceles $A B C_{2} D$, dado o biângulo qualquer $A B C D$

Justificativa. Da figura acima, observamos que os triângulos $B P Q$ e $B P S$ são congruentes pelo caso $L A A_{o}$, análogo ao da Geometria Euclideana, pois $P \hat{B} Q=P \hat{B} S, \overline{B P}$ é lado comum e $P \hat{Q} B=P \hat{S} B$. Logo, $\overline{P Q}=\overline{P S}$. 
Pelo mesmo caso acima, os triângulos $C P S$ e $C P R$ são congruentes, já que $\overline{P C}$ é lado comum, $S \hat{C} P=R \hat{C} P$ e $P \hat{S} C=P \hat{R} C$. Portanto, $\overline{P S}=\overline{P R}$.

Concluímos, deste modo, que o triângulo $P Q R$ é isósceles de base $\overline{Q R}$. Assim, do mesmo modo que na Geometria Euclideana, tem-se que os ângulos adjacentes à base $(P \hat{Q} R$ e $P \hat{R} Q)$ são congruentes. Por conseguinte, $B \hat{Q} R=$ $C \hat{R} Q$, donde segue que $A \hat{Q} R=D \hat{R} Q$, razão pela qual o biângulo $A Q R D$ é isósceles.

Falta apenas provar que, se $A Q R D$ é um biângulo isósceles e $\overline{Q B}=\overline{R C_{2}}$, com $B$ e $C_{2}$ do mesmo lado de $\overline{Q R}$, então $A B C_{2} R$ também é isósceles.

Na figura 4.22, abaixo, tem-se que $M$ é o ponto médio de $\overline{Q R}$ e, por hipótese, $\overline{R C_{2}}=\overline{Q B}$. Além disso, como já provado acima $B \hat{Q} R=C \hat{R} Q=C_{2} \hat{R} Q$. Deste modo, os triângulos $B Q M$ e $C_{2} R M$ são congruentes por $L A L$.

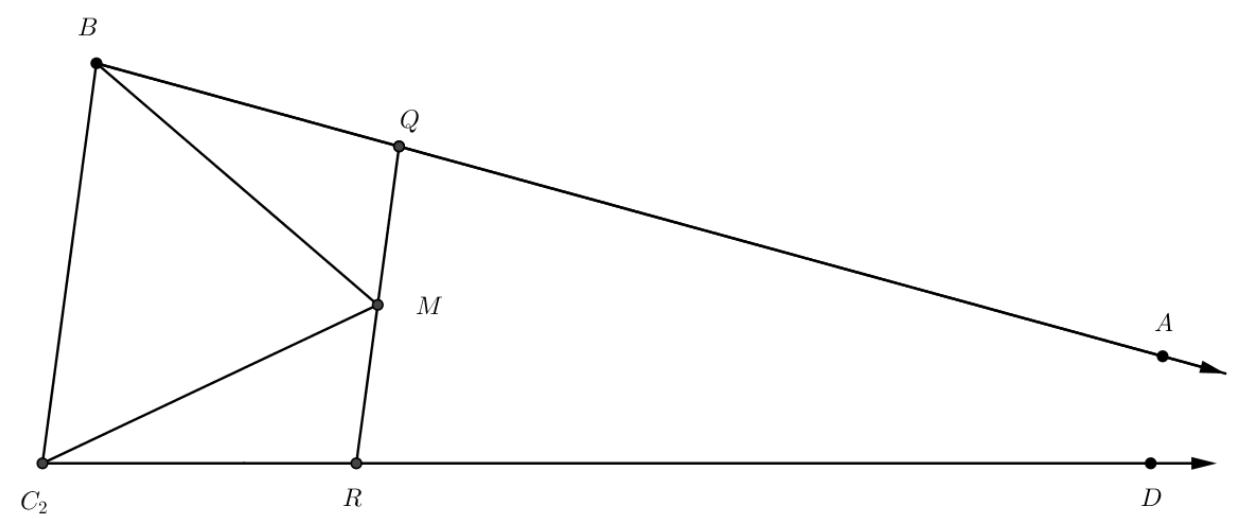

Figura 4.22: Ilustração para justificativa da construção 13

Do exposto, segue que $Q \hat{B} M=R \hat{C}_{2} M$. Ainda, tem-se que $\overline{B M}=\overline{C_{2} M}$, donde se conclui que o triângulo $B M C_{2}$ é isósceles e $M \hat{B} C_{2}=M \hat{C}_{2} B$.

Consequentemente, $Q \hat{B} C_{2}=Q \hat{B} M+M \hat{B} C_{2}=R \hat{C}_{2} M+M \hat{C}_{2} B=R \hat{C}_{2} B$ e o biângulo $A B C_{2} D$ é isósceles.

Agora, podemos apresentar as construções que relacionam $r$ e $\theta$.

Construção 14 Construir, a partir, de um ângulo $\theta$, um segmento hiperbólico de medida r tal que $\tan \theta=2 \sinh \frac{r}{2}$.

Dado o ângulo $\theta=A \hat{B} C$, devemos construir um segmento $\overline{B D}$ perpendicular a $\overrightarrow{B A}$, tal que $C \hat{B} D=\Pi(\overrightarrow{B D})$ e que $D$ e $C$ estejam do mesmo lado de $\overrightarrow{B A}$.

Seja $s$ a reta perpendicular a $\overline{B D}$ e que passa por $D$ (paralela a $\overleftrightarrow{B C}$ ). Devemos, agora, obter, por construção, o ponto $E \in s$ tal que $\overline{B E}=r$ e $C \hat{B} E=B \hat{E} D$. Nestas condições, tem-se que $\tan \theta=2 \sinh \frac{r}{2}$ (figura 4.23). Justificativa. O ponto $D$ pode ser obtido por meio da construção 11. Já o ponto $E$ surge como resultado da construção 13 , que diz respeito à obtenção 


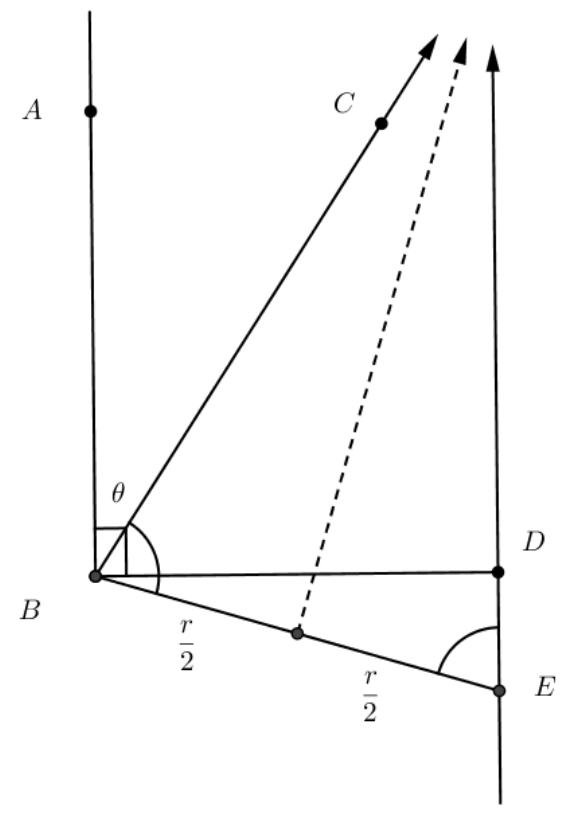

Figura 4.23: Construção do segmento $r$, dado o ângulo $\theta$

de um biângulo fechado e isósceles a partir de um dado vértice de um biângulo fechado qualquer.

Provaremos a seguir, que, a partir construção sugerida acima, obtém-se o segmento $r$ tal que $\tan \theta=2 \sinh \frac{r}{2}$.

Considerando a figura 4.23 e as relações trigonométricas do teorema 17, tem-se que

$$
\tan \theta=\cot \left(\frac{\pi}{2}-\theta\right)=\sinh \overline{B D}
$$

No biângulo isósceles $C B E D$, tem-se que $B \hat{E} D=C \hat{B} E=\Pi\left(\frac{r}{2}\right)$. Por conseguinte, pelos teoremas 14 e 17,

$$
\sin B \hat{E} D=\frac{\sinh \overline{B D}}{\sinh r} \Rightarrow \sin \Pi\left(\frac{r}{2}\right)=\frac{\tan \theta}{\sinh r} \Rightarrow \tan \theta=\sinh r \cdot \sin \Pi\left(\frac{r}{2}\right)
$$

De uma das fórmulas do teorema 17 , sabe-se que $\csc \Pi\left(\frac{r}{2}\right)=\cosh \frac{r}{2}$, donde segue que

$$
\sin \Pi\left(\frac{r}{2}\right)=\frac{1}{\cosh \frac{r}{2}}
$$

Além disso, tem-se que $\sinh r=2 \cdot \sinh \frac{r}{2} \cdot \cosh \frac{r}{2} \quad(3)$. De fato, pela proposição 
19 , tem-se que

$$
\sinh r=\sinh \left(\frac{r}{2}+\frac{r}{2}\right)=\sinh \frac{r}{2} \cdot \cosh \frac{r}{2}+\sinh \frac{r}{2} \cdot \cosh \frac{r}{2}=2 \cdot \sinh \frac{r}{2} \cdot \cosh \frac{r}{2}
$$

Fazendo as substituições pertinentes de (2) e (3) na expressão (1), chegamos a

$$
\tan \theta=2 \cdot \sinh \frac{r}{2} \cdot \cosh \frac{r}{2} \cdot \frac{1}{\cosh \frac{r}{2}} \Rightarrow \tan \theta=2 \cdot \sinh \frac{r}{2}
$$

Construção 15 Construir, a partir de um segmento de medida $r$, um ângulo $\theta$ tal que $\tan \theta=2 \cdot \sinh \frac{r}{2}$.

Pela observação da figura 4.23, não é difícil estabelecer um método de construção para, a partir de $r$, obter $\theta$. Devemos proceder da seguinte forma:

1 - construir um biângulo fechado e isósceles $A B C D$, em que $\overline{B C}=r$. Para tanto, basta utilizar a construção 10 para obter o ângulo de paralelismo associado ao segmento de medida $\frac{r}{2}$;

2 - a partir de $B$, baixar uma perpendicular à reta $\overleftrightarrow{C D}$, que intersectará tal reta no ponto $E$;

3 - traçar a semirreta $\overrightarrow{B F}$, perpendicular a $\overline{B E}$ no ponto $B$. O ângulo $F \hat{B} A$ será o $\theta$ procurado.

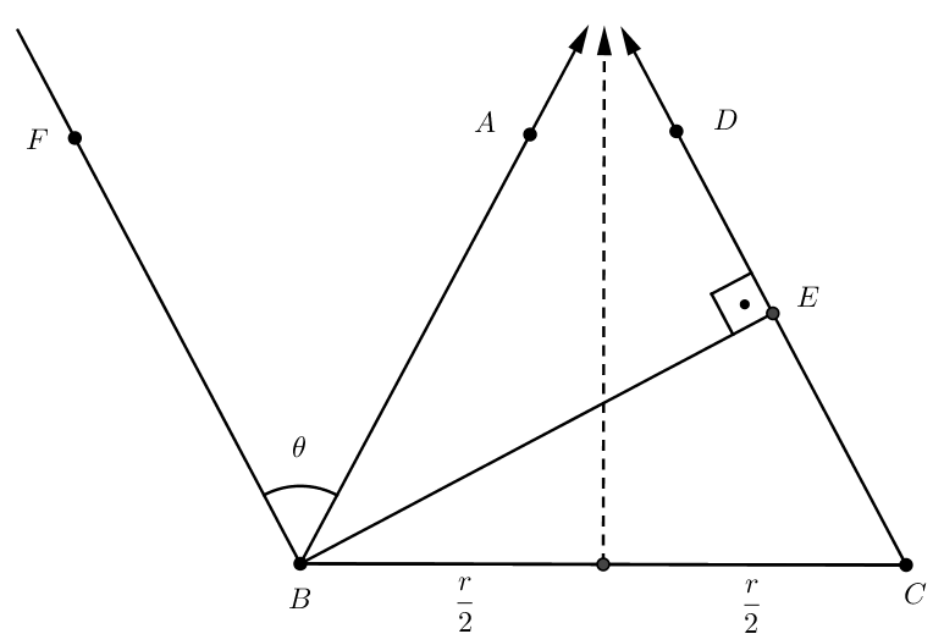

Figura 4.24: Construção do ângulo $\theta$, dado o segmento $r$

Justificativa. Uma vez realizada a construção acima (figura 4.24), é fácil verificar que as mesmas relações trigonométricas referentes à justificativa da construção 14 são válidas, de modo que se obtém o mesmo resultado $\tan \theta=2 \cdot \sinh \frac{r}{2}$. 
Uma última observação: na presente seção do trabalho, será considerada a unidade natural de comprimento da Geometria Hiperbólica, dada pela constante de Schweikart $\left(\Pi(p)=\frac{\pi}{4}\right)$. Observe-se que, conforme provado no Capítulo 3 , subseção 3.1.3, $\sinh p=\cot \frac{\pi}{4}=1$. É comum relacionar a constante de Schweikart com a constante de Gauss $(k)$, escala de distância, de modo que $\sinh (p / k)=1$. No estudo que será feito adiante, será considerado $k=1$.

Feitas as considerações preliminares, cabe esclarecer que a "quadratura do círculo" será estudada mediante a comparação de construtibilidade entre ângulos e comprimentos nos planos hiperbólico $\left(\mathbb{H}^{2}\right)$ e euclideano $\left(\mathbb{E}^{2}\right)$.

O problema relativo à construção de um quadrado e de um círculo de mesma área, em vez de ser considerado do ponto de vista da medida do raio do círculo, será analisado com a utilização do ângulo auxiliar $\theta$, tendo em vista que as construções 14 e 15 permitem obter o ângulo $\theta$ a partir do raio $r$ e vice-versa. A vantagem de proceder desta forma diz respeito ao fato de que um ângulo é construtível no plano hiperbólico se, e somente se, ele pode ser construído com régua e compasso no plano euclideano (teorema 20, provado na subseção 3.1.4 do Capítulo 3). Tal resultado facilita, sobremaneira, a análise do problema da construtibilidade em $\mathbb{H}^{2}$.

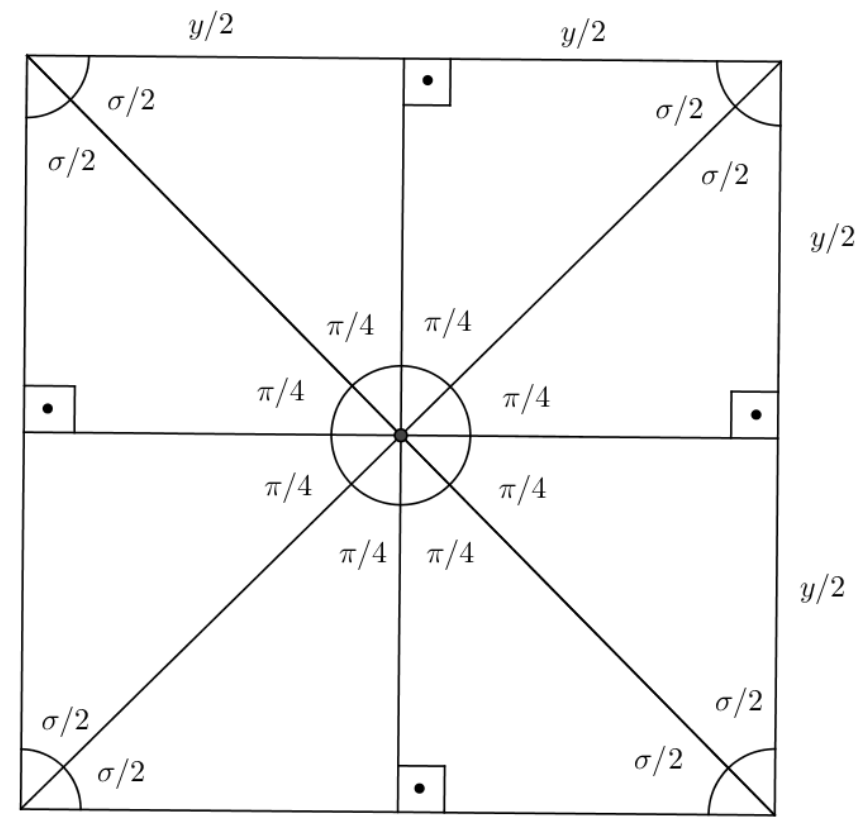

Figura 4.25: Quadrado hiperbólico construído a partir de oito triângulos retângulos congruentes

Finalmente, vamos enunciar e provar os teoremas referentes ao problema da quadratura do círculo na Geometria Hiperbólica.

Em primeiro lugar, devemos notar que, como a área do círculo é dada por $4 \pi \cdot \sinh ^{2}\left(\frac{r}{2}\right)$ e o ângulo $\theta$ é construído mediante a correspondência $\tan \theta=$ 
$2 \sinh \frac{r}{2}$, conclui-se que a área do círculo também é dada por $\pi \cdot \tan ^{2} \theta$.

Ainda, devemos recordar que o quadrado, no plano hiperbólico (figura 4.25), é um quadrilátero com quatro lados congruentes e quatro ângulos internos de mesma medida e agudos. Tal polígono pode ser construído a partir de oito triângulos retângulos congruentes (construção 12), com ângulos internos $\frac{\pi}{4}, \frac{\sigma}{2}$ e $\frac{\pi}{2}$ e um dos catetos de medida $\frac{y}{2}$. Assim, o lado do quadrado tenha medida $y$.

O quadrado construído pelo método acima tem ângulo interno $\sigma$. Logo, considerando que a área de tal quadrado corresponde ao seu defeito, tem-se que esta será dada por $2 \pi-4 \sigma$.

A proposição a seguir nos ensina como relacionar o lado do quadrado $(y)$ com o raio do círculo de mesma área $(r)$.

Proposição 31 Se um quadrado hiperbólico tem lado y e um círculo de mesma área que o quadrado tem raio $r$, então

$$
\cosh y=\tan ^{2}\left(\frac{\pi \cdot \cosh r}{4}\right)
$$

Prova. Considere o quadrado com lado $y$ e ângulo interno $\sigma$, subdivido em oito triângulos retângulos com ângulos $\frac{\sigma}{2}, \frac{\pi}{4}$ e $\frac{\pi}{2}$ (figura 4.25).

De uma das relações da trigonometria hiperbólica (teorema 14), temos que

$$
\cosh \frac{y}{2}=\frac{\cos \frac{\pi}{4}}{\sin \frac{\sigma}{2}}
$$

A área do quadrado é dada por $2 \pi-4 \sigma$ e a do círculo tem fórmula $2 \pi$.( $\cosh r-$ 1). Como as duas figuras têm a mesma área, segue que

$$
2 \pi-4 \sigma=2 \pi \cdot(\cosh r-1)
$$

Dividindo a equação acima por 8, obtemos

$$
\frac{\pi}{4}-\frac{\sigma}{2}=\frac{\pi}{4} \cdot(\cosh r-1) \Rightarrow \frac{\sigma}{2}=\frac{\pi}{2}-\frac{\pi \cdot \cosh r}{4}
$$

Substituindo $(*)$ em $(1)$, chegamos a

$$
\cosh \frac{y}{2}=\frac{\cos \frac{\pi}{4}}{\sin \left(\frac{\pi}{2}-\frac{\pi \cdot \cosh r}{4}\right)}=\frac{\cos \frac{\pi}{4}}{\cos \left(\frac{\pi \cdot \cosh r}{4}\right)}
$$


Mas

$$
\cosh ^{2} \frac{y}{2}=\left(\frac{e^{\frac{y}{2}}+e^{-\frac{y}{2}}}{2}\right)^{2}=\frac{e^{y}+e^{-y}+2}{4}
$$

Logo,

$$
2 \cdot \cosh ^{2} \frac{y}{2}=\cosh y+1 \Rightarrow \cosh y=2 \cdot \cosh ^{2} \frac{y}{2}-1
$$

Por fim, obtemos que

$$
\begin{gathered}
\cosh y=2 \cdot\left(\frac{\frac{\sqrt{2}}{2}}{\cos \left(\frac{\pi \cdot \cosh r}{4}\right)}\right)^{2}-1=2 \cdot \frac{\left(\frac{\sqrt{2}}{2}\right)^{2}}{\cos ^{2}\left(\frac{\pi \cdot \cosh r}{4}\right)}-1 \\
\cosh y=\frac{\sin ^{2}\left(\frac{\pi \cdot \cosh r}{4}\right)}{\cos ^{2}\left(\frac{\pi \cdot \cosh r}{4}\right)}=\tan ^{2}\left(\frac{\pi \cdot \cosh r}{4}\right)
\end{gathered}
$$

Retomando a fórmula do círculo com base no ângulo auxiliar $\theta$, podemos escrever $2 \pi-4 \sigma=\pi \cdot \tan ^{2} \theta$. Deste modo, o problema da construtibilidade de um círculo e de um quadrado de mesma área, em $\mathbb{H}^{2}$, reduz-se à possibilidade de construção dos ângulos $\sigma$ e $\theta$ no plano euclideano, tendo em vista o teorema 20.

Suponha $r$ e $\sigma$ construtíveis em $\mathbb{H}^{2}$. Denotando por $\omega$ a área comum do quadrado e do círculo, tem-se que $\omega=2 \pi-4 \sigma$, de tal modo que $\omega$ é um ângulo que pode ser construído em $\mathbb{E}^{2}$. Por sua vez, $\omega=\pi \cdot \tan ^{2} \theta$ e, como $\theta$ é um ângulo construtível, sua função trigonométrica $\tan \theta$ e, por consequência, $\tan ^{2} \theta$ pertencem a $E$ (conjunto dos comprimentos construtíveis no plano euclideano). Se escrevermos $x=\tan ^{2} \theta$, teremos um ângulo $\omega$ e um segmento $x$ construtíveis em $\mathbb{E}^{2}$, tal que $\omega=\pi x$.

Considerando todas as igualdades já mencionadas, temos

$$
\omega=2 \pi-4 \sigma=\pi \tan ^{2} \theta=\pi x=2 \pi \cdot(\cosh r-1)=4 \pi \sinh ^{2} \frac{r}{2}
$$

Por meio da comparação entre o segundo e o quinto membros acima, chegamos a

$2 \pi-4 \sigma=2 \pi \cdot(\cosh r-1) \Rightarrow \pi-2 \sigma=\pi \cosh r-\pi \Rightarrow 2 \sigma+\pi \cosh r=2 \pi$

Já, da igualdade entre o quarto e o quinto membros, obtemos

$$
\pi x=2 \pi \cdot(\cosh r-1) \Rightarrow x=2 \cosh r-2 \Rightarrow x+2=2 \cosh r
$$


Para demonstrarmos o primeiro teorema relativo à construtibilidade de um quadrado e de um círculo de mesma área no plano hiperbólico, provaremos, primeiramente, uma proposição com o auxílio de um teorema que diz respeito a números transcendentes, enunciado adiante. Antes, porém, será necessário apresentar um comentário sobre números algébricos e transcendentes.

É sabido que o conjunto $\mathbb{A}$, dos números algébricos, é um corpo que, dentre outros, tem como elemento $i$ tal que $i^{2}=-1$. O interesse de nos reportarmos aos números algébricos diz respeito ao fato de que os comprimentos construtíveis no plano euclideano são números algébricos, ou seja, $E \subset \mathbb{A}$. Logo, não há comprimentos representados por números transcendentes que possam ser construídos com régua e compasso no plano euclideano. Ademais, $E(i)=\{a+b i ; a, b \in E\}$, extensão de corpo de $E$ por $i$, é um subcorpo de $\mathbb{A}$.

Teorema 21 (Gelfond-Schneider) Se $\varphi$ e $\chi$ são dois números algébricos diferentes de $0, \varphi \neq 1$ e $\chi \notin \mathbb{Q}$, então qualquer valor de $\varphi^{\chi}$ é transcendente.

É importante observar que $\varphi^{\chi}=e^{\chi \ln (\varphi)}$, donde se conclui tal $\varphi^{\chi}$ é multivaluado como logaritmo complexo. Deve-se destacar, também, que o resultado acima tem sua aplicação não apenas quando $\chi \in \mathbb{R}$, mas também quando o expoente referido é complexo com parte imaginária não nula (por exemplo $i^{-2 i}$ tem como um valor $e^{\pi}$, donde segue que $e^{\pi}$ é transcendente). Note-se, também, que a base do número considerado $(\varphi)$ não pode ser igual 1 , mas o teorema não impede que se tenha $\varphi=-1$.

Uma apresentação do teorema de Gelfond-Schneider, com detalhes, pode ser encontrada em [6], pp. 134-150.

Com base no teorema 21, provaremos a seguinte proposição:

Proposição 32 Se $\omega=\pi x$ é um ângulo que pode ser construído com régua e compasso no plano euclideano e $x \in E$, então $x \in \mathbb{Q}$.

Prova. Suponha que $\omega=\pi x$ é um ângulo construtível em $\mathbb{E}^{2}$. Então, $\cos \pi x$ e $\sin \pi x$ pertencem a $E$. Logo, $e^{i \pi x}=\cos \pi x+i \cdot \sin \pi x \in E(i) \subset \mathbb{A}$. Tomando $\ln (-1)=i \pi$, segue que $(-1)^{x}=e^{i \pi x}$, de tal modo que $(-1)^{x}$ é um número algébrico.

Tendo em vista que o comprimento $x$, por hipótese, pode ser construído com régua e compasso no plano euclideano, conclui-se que $x \in \mathbb{A}$. Daí, como $-1 \mathrm{e}$ $x$ são algébricos e $(-1)^{x} \in \mathbb{A}$, segue, pelo Teorema de Gelfond-Schneider, que $x$ só pode ser racional.

Pela proposição provada acima e pelo Teorema 20, tem-se que, se $\omega=\pi x$ é construtível no plano hiperbólico e $x$ é um comprimento que pode ser construído com régua e compasso em $\mathbb{E}^{2}$, então $x$ é racional. Ademais, da 
relação (2), exposta anteriormente nesta subseção $(x+2=2 \cosh r)$, concluise que, como $x \in \mathbb{Q}, \cosh r$ também é racional.

Agora, estamos em condições de enunciar e provar o primeiro teorema referente à construtibilidade no plano hiperbólico.

Teorema 22 (Nestorovich-Jagy) Considere, em $\mathbb{H}^{2}$, um quadrado com ângulo interno $\sigma$ e um círculo de mesma área, de raio $r$. Então, ambos são construtiveis se, e somente se, $0 \leq \sigma<\frac{\pi}{2}$ e $\sigma$ é um múltiplo inteiro de $\frac{2 \pi}{n}$, em que $n$ é um número inteiro positivo tal que o polígono regular de $n$ lados possa ser construído com régua e compasso no plano euclideano.

Prova. Suponhamos, primeiramente, $\sigma$ e $r$ construtíveis em $\mathbb{H}^{2}$.

Como $r$ é construtível no plano hiperbólico, podemos obter $\theta$ pela construção 15. Assim, $\theta$ também pode ser construído com régua e compasso em $\mathbb{E}^{2}$ (teorema 20), donde se conclui que $\tan \theta \in E$ e também que $\tan ^{2} \theta \in E$.

Além disso, afirmamos que $\omega=2 \pi-4 \sigma$ é um ângulo construtível no plano hiperbólico. De fato, como $\sigma$ é construtível em $\mathbb{H}^{2}$, também pode ser construído com régua e compasso em $\mathbb{E}^{2}$ (teorema 20). Portanto, $\sin \sigma \in E$ e $\cos \sigma \in E$. Daí, segue que

$$
\begin{aligned}
\sin \omega= & \sin (2 \pi-4 \sigma)=-\sin 4 \sigma=-2 \sin 2 \sigma \cdot \cos 2 \sigma \Rightarrow \\
& \Rightarrow \sin \omega=-4 \sin \sigma \cdot \cos \sigma \cdot\left(\cos ^{2} \sigma-\sin ^{2} \sigma\right)
\end{aligned}
$$

Por conseguinte, $\sin \omega \in E$, pois pode ser obtido por meio das construções vistas na prova do teorema 1 a partir de $\sin \sigma$ e $\cos \sigma$, que estão em $E$. Consequentemente, $\omega$ é construtível no plano euclideano e, pelo teorema 20, $\omega$ também pode ser construído com régua e compasso em $\mathbb{H}^{2}$.

Mas $\omega=\pi \tan ^{2} \theta$ e $\tan ^{2} \theta \in E$. Por conseguinte, $x=\tan ^{2} \theta \in \mathbb{Q}$, tendo em vista a proposição 32 .

Seja $x=\frac{m}{n}, m, n \in \mathbb{Z}_{+}^{*}, \operatorname{com} \operatorname{mdc}(m, n)=1$. Assim, existem inteiros $u$ e $v$ tais que $u m+v n=1$. Multiplicando ambos os membros da equação anterior por $\pi / n$, obtemos

$$
u m \frac{\pi}{n}+v \pi=\frac{\pi}{n} \Rightarrow u \omega+v \pi=\frac{\pi}{n}
$$

Prova-se, pelo princípio da indução finita, que se $\alpha$ é um ângulo construtível, então $p \alpha$ também é construtível, $p \in \mathbb{N}$. Como, por hipótese, $\alpha$ pode ser construído com régua e compasso, então $\sin \alpha \in E$ e $\cos \alpha \in E$. Para $p=2$, tem-se que

$$
\sin 2 \alpha=\sin (\alpha+\alpha)=2 \cdot \sin \alpha \cdot \cos \alpha
$$


Assim, vê-se que $\sin 2 \alpha$ pode ser construído com régua e compasso em $\mathbb{E}^{2}$ a partir de $\sin \alpha$ e $\sin \alpha$, considerando as construções vistas na prova do teorema 1.

Suponha, agora, que $p \alpha$ é construtível. Para $p+1$, tem-se que

$$
\sin [(p+1) \cdot \alpha]=\sin (p \alpha+\alpha)=\sin p \alpha \cdot \cos \alpha+\cos p \alpha \cdot \sin \alpha
$$

Do mesmo modo, $\sin [(p+1) . \alpha]$ pode ser obtido, com régua e compasso, a partir de $\sin \alpha, \cos \alpha, \cos p \alpha$ e $\sin p \alpha$ que, por hipótese, estão em $E$.

Retomando a demonstração, concluiremos, a partir da equação $u \omega+v \pi=\frac{\pi}{n}$, com $u, v \in \mathbb{Z}$, que $\frac{\pi}{n}$ é construtível em $\mathbb{E}^{2}$ (e, consequentemente, em $\mathbb{H}^{2}$ ). Para verificar este fato, basta observar que

$$
\sin \frac{\pi}{n}=\sin u \omega \cdot \cos v \pi+\sin v \pi \cdot \cos u \omega=\sin u \omega \cdot \cos v \pi
$$

de modo que se tem $\sin \frac{\pi}{n}=\sin u \omega$ ou $\sin \frac{\pi}{n}=-\sin u \omega$, de acordo com a patidade de $v$. Como $\omega$ é construtível em $\mathbb{E}^{2}, u \omega$ também pode ser construído com régua e compasso, donde segue que as funções trigonométricas deste último ângulo estão em $E$ e, por via de consequência, o ângulo $\frac{\pi}{n}$ é construtível.

A possibilidade de se construir o ângulo $\frac{\pi}{n}$ tem relação com o teorema 6 (GaussWentzel), no seguinte sentido: desenhando, no interior de um círculo, o ângulo central de medida $\frac{2 \pi}{n}$ e copiando este ângulo $n-1$ vezes, construímos o polígono regular de $n$ lados no plano euclideano. A construção referida é possível se, somente se, $n=2^{k} \cdot F_{i_{1}} \cdot F_{i_{2}} \ldots F_{i_{r}}$ em que os $F_{i_{j}}$ são números primos de Fermat. Portanto, para que o ângulo $\frac{\pi}{n}$ seja construtível, devemos ter $n$ da forma $n=2^{k} \cdot F_{i_{1}} \cdot F_{i_{2}} \ldots F_{i_{r}}$ em que os $F_{i_{j}}$ são números primos de Fermat. Note que o referido ângulo pode ser reescrito como $\frac{2 \pi}{2 n}=\frac{2 \pi}{n^{\prime}}$, em que $n^{\prime}$ continua sendo um número da forma do teorema 6.

Mas $n$ é o denominador de $\omega=\pi x=\frac{m \pi}{n}$. Logo, $\omega=m \cdot \frac{2 \pi}{n^{\prime}}$, em que $n^{\prime}$ corresponde ao número de lados de um polígono construtível com régua e compasso no plano euclideano.

Daí, como $\omega=2 \pi-4 \sigma$, segue que

$$
\sigma=\frac{2 \pi-\omega}{4}=\frac{2 \pi-m \cdot \frac{2 \pi}{n^{\prime}}}{4}=\left(n^{\prime}-m\right) \cdot \frac{2 \pi}{4 n^{\prime}}=\left(n^{\prime}-m\right) \cdot \frac{2 \pi}{n^{\prime \prime}}
$$

Observe que, como $\omega=m \cdot \frac{2 \pi}{n^{\prime}}$ é a área do quadrado e do círculo, devemos ter $\omega<2 \pi$, donde se conclui que $m<n^{\prime}$ e $n^{\prime}-m>0$. 
Logo, $\sigma$ é da forma $\frac{2 k \pi}{n^{\prime \prime}}$ em que $k \in \mathbb{Z}_{+}^{*}$ e em que $n^{\prime \prime}$ é o número de lados de um polígono que pode ser construído com régua e compasso no plano euclideano. Reciprocamente, seja $\sigma=\frac{2 k \pi}{n}$, tal que $k \in \mathbb{Z}_{+}^{*}, n$ é da forma $n=$ $2^{k} \cdot F_{i_{1}} \cdot F_{i_{2}} \ldots F_{i_{r}}$ em que os $F_{i_{j}}$ são números primos de Fermat.

Neste caso, $\sigma$ é construtível tanto em $\mathbb{E}^{2}$ quanto em $\mathbb{H}^{2}$ (teorema 20). Do mesmo modo, $\omega=2 \pi-4 \sigma$, pode ser construído com régua e compasso nos planos euclideano e hiperbólico, como já visto na primeira parte da demonstração. Além disso,

$$
\omega=2 \pi-4 \cdot\left(\frac{2 k \pi}{n}\right)=2 \cdot\left(\frac{n-4 k}{n}\right) \cdot \pi
$$

Daí, segue que $\omega$ é um múltiplo racional de $\pi$. Mas $\omega=\pi \tan ^{2} \theta$, donde se conclui que $\tan ^{2} \theta \in \mathbb{Q}$ e, por consequência, $\tan ^{2} \theta \in E$ e $\tan \theta \in E$. Logo, $\theta$ é um ângulo construtível no plano euclideano e também no plano hiperbólico (teorema 20).

Deste modo, podemos utilizar a construção 14 para obter o raio $r$ do círculo. Assim, conclui-se que o círculo de mesma área que o quadrado pode ser construído com régua e compasso no plano hiperbólico.

Para finalizar a demonstração, falta apenas esclarecer que podemos considerar quadrados de lado infinito e ângulo $\sigma=0$, mas não faz sentido considerar quadrados de ângulo interno $\pi / 2$, já que tais figuras, no plano hiperbólico, seriam um único ponto, com área 0 . Logo, $0 \leq \sigma<\pi / 2$.

O teorema acima, de forma surpreendente, estabelece que o problema da construção de um círculo e um quadrado de mesma área, no plano hiperbólico, é equivalente ao problema da construtibilidade de polígonos com régua e compasso no plano euclideano (ou mesmo no plano hiperbólico; observação após a prova do teorema 20, na subseção 3.1.4).

O teorema 22 produziu, simultaneamente, o quadrado e o círculo de mesma área com as informações dadas. No entanto, é possível indagar se existe um método para, a partir de $r$, obter $\sigma$ ou se, iniciando por $\sigma$, existe um método para construir $r$. Como já se podia imaginar pelos exemplos apresentados na seção 4.1, a resposta a tais perguntas é negativa. Nas demonstrações dos teoremas seguintes, serão apresentadas classes de exemplos em que, partindo do ângulo auxiliar $\theta$, obtém-se $\sigma$ não construtível e vice-versa.

Teorema 23 (Jagy) Não existe método geral de construção com régua e compasso, no plano hiperbólico, que, a partir do raio $r$ do círculo, produza o ângulo interno $\sigma$ do quadrado que tem mesma área que o círculo dado. 
Prova. Suponha que exista um método geral para, a partir de qualquer raio de círculo construtível, produzir o correspondente ângulo interno $\sigma$ do quadrado de mesma área.

Considere o raio $r$ do círculo que produza o ângulo $\theta=\arctan \left(\frac{m}{n}\right)$, tal que $m$ e $n$ são primos entre si e $n$ não é potência de 2 , mas tem algum fator primo ímpar $d$. Deste modo, o ângulo $\theta$ é construtível em $\mathbb{E}^{2}$ e em $\mathbb{H}^{2}$ (teorema 20) e o círculo tem área $\omega=\pi \tan ^{2} \theta=\pi \cdot m^{2} / n^{2}$.

Como $\operatorname{mdc}(m, n)=1$, tem-se que $\operatorname{mdc}\left(m^{2}, n^{2}\right)=1$. Deste modo, existem inteiros $x$ e $y$ tais que $x \cdot m^{2}+y \cdot n^{2}=1$. Multiplicando a referida relação por $\frac{\pi}{n^{2}}$ e procedendo de modo análogo ao que foi feito na primeira parte da demonstração do teorema 22 , concluímos que, se $\omega$ é construtível (em $\mathbb{E}^{2}$ e em $\left.\mathbb{H}^{2}\right)$, então $\frac{\pi}{n^{2}}$ é construtível.

Mas $\frac{\pi}{n^{2}}$ não pode ser construído com régua e compasso, pois, caso contrário, seria possível construir um polígono regular de $n^{2}$ lados, com $n$ da forma acima, o que é impossível pelo teorema 6 . Consequentemente, $\omega$ não é construtível. Daí, segue que, como $\sigma=\frac{2 \pi-\omega}{4}$, tal ângulo também não é construtível (contradição). Logo, não existe o método de construção referido.

Jagy, em seu artigo [4], mencionou que Nestorovich, o primeiro matemático a descobrir o teorema 22 , apresentou um exemplo em que

$$
\sinh \left(\frac{r}{2}\right)=\frac{1}{2} \sqrt{2-\sqrt{2}} \Rightarrow \theta=\arctan (\sqrt{2-\sqrt{2}})
$$

donde se conclui que $r$ e $\theta$ são construtíveis. Porém, o ângulo interno $\sigma$, em tal hipótese, não possibilita a construção do quadrado com mesma área que o círculo, pois a área é $\omega=\pi \cdot(2-\sqrt{2})$ e

$$
\sigma=\frac{2 \pi-\omega}{4} \Rightarrow \frac{2 \pi-2 \pi+\pi \sqrt{2}}{4} \Rightarrow \sigma=\pi \frac{\sqrt{2}}{4}
$$

Com base em tal exemplo, concluiu-se que a classe dos "quadrados circuláveis" é mais ampla que a dos "círculos quadráveis".

Antes de enunciarmos e provarmos o próximo teorema, introduziremos um teorema também apresentado em [6], p. 41.

Teorema 24 (Olmsted) Se $\tau$ é um múltiplo racional de $\pi$, então os únicos valores pertencentes a $\mathbb{Q}$ para $\tan \tau$ são 0,1 e -1 .

Com o auxílio do resultado acima, demonstraremos o último teorema sobre a construtibilidade de um círculo e de um quadrado de mesma área no plano hiperbólico. 
Teorema 25 (Jagy) Não existe método geral de construção com régua e compasso, no plano hiperbólico, que, a partir do ângulo interno $\sigma$ do quadrado, produza o raio $r$ do círculo que tem mesma área que o quadrado.

Prova. Suponha que exista um método para, partindo de $\sigma$ construtível, obter $r$ por construção. Então, o método deve permitir a construção de $r$ a partir de qualquer $\sigma$ considerado.

Seja $q \in \mathbb{Q}$ tal que $q>0$ e $q \neq 1$. Assim, o ângulo $\sigma=\arctan q$ pode ser construído com régua e compasso no plano hiperbólico, já que $q$ é construtível em $\mathbb{E}^{2}$ (teorema 20). Tendo em vista que $\sigma$ é agudo, obtemos, das relações trigonométricas, o seguinte:

$$
\begin{aligned}
& \tan ^{2} \sigma=\frac{\sin ^{2} \sigma}{\cos ^{2} \sigma}=\frac{\sin ^{2} \sigma}{1-\sin ^{2} \sigma} \Rightarrow \sin ^{2} \sigma=\frac{\tan ^{2} \sigma}{1+\tan ^{2} \sigma} \Rightarrow \sin \sigma=\frac{\tan \sigma}{\sqrt{1+\tan ^{2} \sigma}} \\
& \cos ^{2} \sigma=1-\sin ^{2} \sigma=1-\frac{\tan ^{2} \sigma}{1+\tan ^{2} \sigma}=\frac{1}{1+\tan ^{2} \sigma} \Rightarrow \cos \sigma=\frac{1}{\sqrt{1+\tan ^{2} \sigma}}
\end{aligned}
$$

Fazendo a substituição $\tan \sigma=q$, chegamos a $\sin \sigma=\frac{q}{\sqrt{1+q^{2}}}$ e $\cos \sigma=$ $\frac{1}{\sqrt{1+q^{2}}}$. Deste modo, $\sin \sigma \in E$ e $\cos \sigma \in E$, donde segue que $e^{i \sigma}=$ $\sin \sigma+i \cos \sigma \in E(i) \subset \mathbb{A}$.

Pelo teorema de Olmsted, como tan $\sigma=q$ e $q$ é racional positivo diferente de 1 , $\frac{\sigma}{\pi}$ é um número irracional. Tomando $e^{i \pi}=-1$, tem-se $(-1)^{\frac{\sigma}{\pi}}=\left(e^{i \pi}\right)^{\frac{\sigma}{\pi}}=$ $e^{i \sigma} \in \mathbb{A}$. Consequentemente, $\frac{\sigma}{\pi}$ é um número transcendente, tendo em vista o teorema de Gelfond-Schneider, pois -1 e $(-1)^{\frac{\sigma}{\pi}}$ são números algébricos e $\frac{\sigma}{\pi} \notin \mathbb{Q}$.

Uma vez que $\omega=2 \pi-4 \sigma$, temos $\frac{\omega}{\pi}=2-\frac{4 \sigma}{\pi}$, donde resulta que $\frac{\omega}{\pi}$ também é transcendente. Mas, $\frac{\omega}{\pi}=x=\tan ^{2} \theta$, donde se conclui que $\tan ^{2} \theta$ e, finalmente, $\tan \theta$ não são construtíveis. Daí, segue que não é possível construir $\theta$ com régua e compasso, e, também, não é possível obter $r$ por construção, o que está em contradição com a hipótese. Portanto, não existe o método mencionado.

Retomando os exemplos apresentados na seção 4.1, observamos que, de fato, os ângulos para os quais foi possível a construção do quadrado e do círculo de mesma área foram $\frac{\pi}{6}$ (exemplo construído) e $\frac{\pi}{4}$ (exemplo 1 ), os quais são ângulos centrais associados a polígonos de 12 e 8 lados, que podem ser construídos no plano euclideano pelo teorema 6 (Gauss-Wentzel). Portanto, como já era de se esperar, tais ângulos estão de acordo com o teorema 22 (Nestorovich-Jagy). 
No exemplo 2, partimos de um raio $r$ construtível em $\mathbb{H}^{2}$, mas obtemos o ângulo interno $\frac{\pi}{9}$ do quadrado de mesma área, que pode ser reescrito como $\frac{2 \pi}{18}$. O referido ângulo tem denominador que, na forma fatorada, é $2 \times 3^{2}$ e não corresponde à fatoração do número de lados de um polígono construtível em $\mathbb{E}^{2}$ (teorema 6, de Gauss-Wentzel). Consequentemente, não é possível construir o quadrado que tenha tal ângulo interno, tendo em vista que este não satisfaz o enunciado do teorema 22.

Por fim, no exemplo 3 , em que $\sigma=\arctan \frac{3}{4}$, verificamos que não é possível construir o círculo que tenha mesma área que o quadrado com tal ângulo interno, tendo em vista os teoremas 22 e 25. É interessante recordar que, quando tentamos obter o raio euclideano do círculo, no modelo do disco de Poincaré, com base na fórmula da proposição 30, chegamos à relação

$$
\frac{1}{x^{2}}=\frac{\pi}{\arctan \frac{4}{3}}-1
$$

Como visto na demonstração do teorema 25, podemos concluir, pelos teoremas 24 (Olmsted) e 21 (Gelfond-Schneider), que o número

$$
\frac{\pi}{\arctan \frac{4}{3}}
$$

é transcendente, donde segue que $\frac{1}{x^{2}}$ e finalmente $x$ são transcendentes. Logo, mais uma vez, concluímos que não é possível realizar a construção do círculo que tem mesma área que o quadrado com ângulo interno $\arctan \frac{3}{4}$. 


\section{Referências bibliográficas}

[1] BONOLA R. Non-Euclidean Geometry. Dover Publications, New York, 1st edition, 1955.

[2] DAVIS, N. Squaring the circle in the hyperbolic disk. Rose-Hulman Undergraduate Mathematics Journal, Vol. 15, № 1, Spring:p. 22-30, 2014.

[3] RAMSAY, A.; RICHTMYER; R. D. Introduction do Hyperbolic Geometry. Springer-Verlag, New York, 1995.

[4] JAGY, W. C. Squaring circles in the hyperbolic plane. The Mathematical Intelligencer, Vol. 17, № 2:p. 31-36, 1995.

[5] PRESSLEY, A. N. Elementary Differential Geometry. Springer-Verlag, London, 2001.

[6] NIVEN, I. Irrational Numbers. The Mathematical Association of America, Rahway, New Jersey, 1956.

[7] BOYER, C. B.; MERZBACH, U. C. História da Matemática. Editora Edgard Blucher, São Paulo, 3 ed., 2012.

[8] BIRKHOFF, G.; MACLANE S. A Survey of Modern Algebra. Macmillan Publishing, New York, 4th edition, 1977.

[9] HARTSHORNE, R. Geometry: Euclid and Beyond. Springer-Verlag, New York, 2000.

[10] OLIVEIRA, J. M. A irracionalidade e a transcendência do número $\pi$. Dissertação de mestrado (modalidade profissional - profmat), Instituto de Geociências e Ciências Exatas, Universidade Estadual Paulista, São Paulo, 2013.

[11] GREENBERG, M. J. Euclidean and Non-Euclidean Geometries Development and History. W. H. Freeman and Company, New York, 3rd edition, 1994.

[12] MARTIN, G. E. The Foundations of Geometry and the NonEuclidean Plane. Springer-Verlag, New York, 1975. 
[13] BARBOSA, J. L. M. Geometria Hiperbólica. Associação Instituto Nacional de Matemática Pura e Aplicada - IMPA, Rio de Janeiro, 3aimpressão, 2007.

[14] DELGADO, J.; FRENSEL, K.; CRISSAFF L.. Geometria Analítica (Coleção PROFMAT). Sociedade Brasileira de Matemática, Rio de Janeiro, 1 ed., 2013.

[15] DOMInGuES, H. H.; IEZZI G. Álgebra Moderna. Atual Editora, São Paulo, 2 ed., 1982.

[16] HEFEZ, A.; FERNANDEZ, C. S. Introdução à Álgebra Linear (Coleção PROFMAT). Sociedade Brasileira de Matemática, Rio de Janeiro, 2 ed., 2016.

[17] MUniz NeTO, A. C. Geometria (Coleção PROFMAT). Sociedade Brasileira de Matemática, Rio de Janeiro, 1 ed., 2013. 